Florida International University

FIU Digital Commons

FIU Electronic Theses and Dissertations

University Graduate School

$6-12-2018$

\title{
Predicting the Geographic Origin of Heroin by Multivariate Analysis of Elemental Composition and Strontium Isotope Ratios
}

Joshua S. DeBord

Florida International University, jsdebord@gmail.com

DOI: 10.25148 /etd.FIDC006831

Follow this and additional works at: https://digitalcommons.fiu.edu/etd

Part of the Analytical Chemistry Commons

\section{Recommended Citation}

DeBord, Joshua S., "Predicting the Geographic Origin of Heroin by Multivariate Analysis of Elemental Composition and Strontium Isotope Ratios" (2018). FIU Electronic Theses and Dissertations. 3802.

https://digitalcommons.fiu.edu/etd/3802 
FLORIDA INTERNATIONAL UNIVERSITY

Miami, Florida

PREDICTING THE GEOGRAPHIC ORIGIN OF HEROIN BY MULTIVARIATE ANALYSIS OF ELEMENTAL COMPOSITION AND STRONTIUM ISOTOPE RATIOS

A dissertation submitted in partial fulfillment of the requirements for the degree of DOCTOR OF PHILOSOPHY

in

CHEMISTRY

by

Joshua DeBord

2018 
To: Dean Michael R. Heithaus

College of Arts, Sciences and Education

This dissertation, written by Joshua DeBord, and entitled Predicting the Geographic Origin of Heroin by Multivariate Analysis of Elemental Composition and Strontium Isotope Ratios, having been approved in respect to style and intellectual content, is referred to you for judgment.

We have read this dissertation and recommend that it be approved.

$\begin{array}{r}\hline \text { Yong Cai } \\ \hline \text { Anthony DeCaprio } \\ \hline \text { Joong-Ho Moon } \\ \hline \text { José Almirall, Major Professor }\end{array}$

Date of Defense: June 12, 2018

The dissertation of Joshua DeBord is approved.

Dean Michael R. Heithaus

College of Arts, Sciences and Education

Andrés G. Gil

Vice President for Research and Economic Development and Dean of the University Graduate School

Florida International University, 2018 
(C) Copyright 2018 by Joshua S. DeBord

All rights reserved. 


\section{DEDICATION}

To my wife, Jacqueline Noelle DeBord

And to my grandfather, Kenneth Dale Jackson 


\section{ACKNOWLEDGMENTS}

My dissertation and the research described herein could not have been possible if not for my mentor, Professor José R. Almirall. I owe so much of my success to him for accepting me into his research group and trusting me to conduct this important research project. His support and guidance have been invaluable in overcoming the challenges and discovering the messages hidden amidst the compendium of data that I gathered. I am also so thankful to the Almirall family and to former and current "A-Team" research group members for the lasting friendships we have built during my $\mathrm{PhD}$.

The United States Drug Enforcement Administration Special Testing and Research Laboratory, especially scientists Sini Panicker and Tom Duncan have been vital to performing this research and providing guidance on the Heroin Signature Program's methods and duties. The United States Drug Enforcement Administration and Counter Terrorism Technical Support Office deserve special recognition for design of the project requirements and administration of the contract to perform the research, respectively.

I must give my most sincere thanks to Dr. Ali Pourmand, Dr. Sarah Jantzi, Professor Rosemary Hickey-Vargas, Cassie-Jo McBride BS and Jerome Mulloor MSFS for their efforts to support me on this research project. Dr. Pourmand has been like a second mentor to me and an invaluable collaborator, guiding me through the isotope studies described in the dissertation and granting me access to conduct the isotope exchange chemistry in his lab. Dr. Jantzi has helped me in areas of planning, method design, data analysis and reporting. She also absorbed a significant amount of the stress that I would have otherwise felt from managing all of the obligations to this research, my $\mathrm{PhD}$ requirements and to my 
family. I cannot thank committee member, Professor Hickey-Vargas enough for the private use of one of her research laboratories and clean lab in which I performed all of my sample dissolution, and also for her accommodation of the necessary equipment to do so. Ms. McBride was at my right hand for nearly all of the sample preparation and instrumental analysis. She was so responsible, helpful and patient in all of the ways necessary for conducting research on such a dangerous controlled substance. Mr. Mulloor was vital to assisting me with sample preparation in the later stages of the research and in conducting the headspace-sampling portion of the research project.

Finally, I want to thank my other dissertation committee members, Dr. Yong Cai, Dr. Anthony DeCaprio and Dr. Joong-Ho Moon for providing me valuable support, criticism and encouragement during my studies through their thoughtful questions, advice and letters of recommendation. I gratefully acknowledge the University Graduate School at Florida International University for its financial support through my $\mathrm{PhD}$ research with graduate assistantships and by awarding me with the dissertation year fellowship. The contracted funding for the research was awarded by the Counter Terrorism Technical Support Office, IS-FI-4174. 


\begin{abstract}
OF THE DISSERTATION
PREDICTING THE GEOGRAPHIC ORIGIN OF HEROIN BY MULTIVARIATE ANALYSIS OF ELEMENTAL COMPOSITION AND STRONTIUM ISOTOPE RATIOS
\end{abstract}

by

Joshua DeBord

Florida International University, 2018

Miami, Florida

Professor José Almirall, Major Professor

The goal of this research was to aid in the fight against the heroin and opioid epidemic by developing new methodology for heroin provenance determination and forensic sample comparison. Over 400 illicit heroin powder samples were analyzed using quadrupole and high-resolution inductively coupled plasma-mass spectrometry (Q-ICPMS and HR-ICP-MS) to measure and to identify elemental contaminants useful for associating heroin samples of common origin and differentiating heroin of different geographic origins. Additionally, 198 heroin samples were analyzed by multi-collector ICP-MS (MC-ICP-MS) to measure radiogenic strontium isotope ratios $\left({ }^{87} \mathrm{Sr} /{ }^{86} \mathrm{Sr}\right)$ with high-precision for heroin provenance determination, for the first time.

Supervised discriminant analysis models were constructed to predict heroin origin using elemental composition. The model was able to correctly associate $88 \%$ of the samples to their region of origin. When ${ }^{87} \mathrm{Sr} /{ }^{86} \mathrm{Sr}$ data were combined with Q-ICP-MS 
elemental data, the correct association of heroin samples improved to $\geq 90 \%$ for all groups with an average of $93 \%$ correct classification.

For forensic sample comparisons, quantitative elemental data (11 elements measured) from 120 samples, 30 from each of the four regions, were compared to assess the rate of discrimination (5400 total comparisons). Using a match criterion of \pm 3 standard deviations about the mean, only 14 of the 5400 possible comparison pairs were not discriminated resulting in a discrimination rate of $99.7 \%$. For determining the rate of correct associations, three replicates of 24 duplicate samples were prepared and analyzed on separate days. Only one of the 24 correct pairs were not associated for a correct association rate of $95.8 \%$. New methods for provenance determination and sample comparison are expected to be incredibly useful to intelligence agencies and law enforcement working to reduce the proliferation of heroin.

Additional research performed includes evaluation of boron isotope ratios for provenance determination, assessment of the contribution of adulterants to the elemental profile of simulated street-level heroin and analysis of the volatile and semi volatile organic compounds of heroin for the purpose of sample profiling. 


\section{TABLE OF CONTENTS}

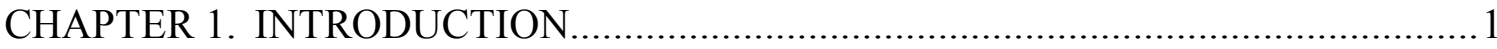

1.1 Statement of the Problem ................................................................................

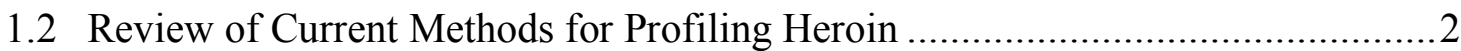

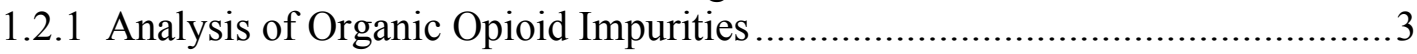

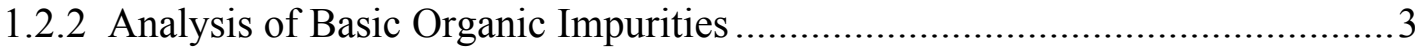

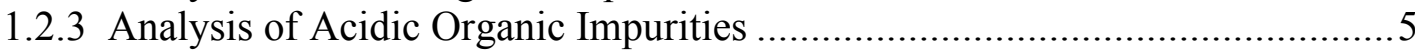

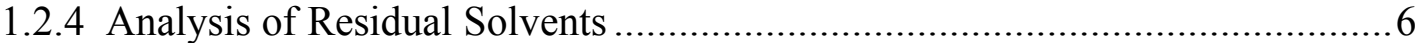

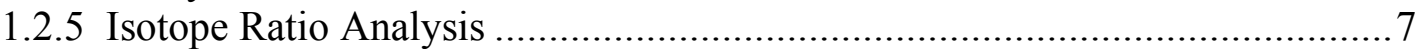

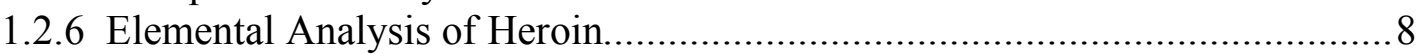

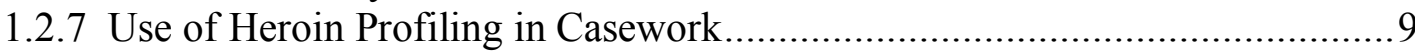

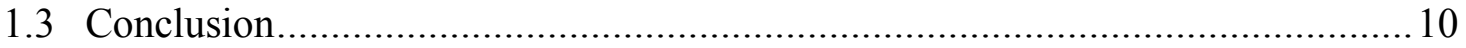

CHAPTER 2. ELEMENTAL ANALYSIS OF HEROIN ….......................................... 12

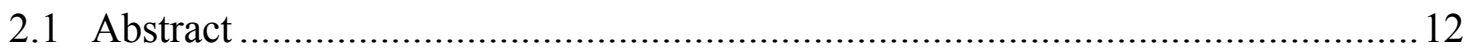

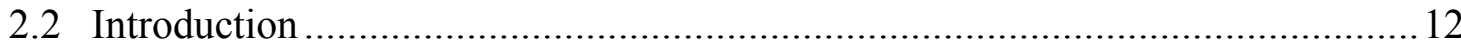

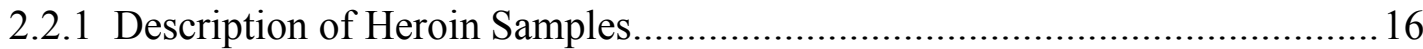

2.2.2 Inductively Coupled Plasma-Mass Spectrometry .......................................... 18

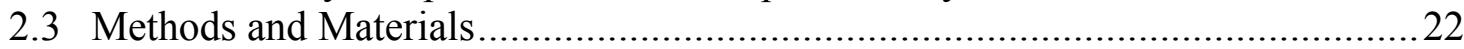

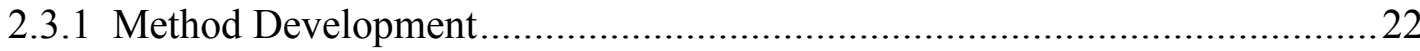

2.3.2 Testing for Method Robustness ……………..............................................2

2.3.3 Micro-wave Assisted Acid Digestion for Elemental Analysis........................29

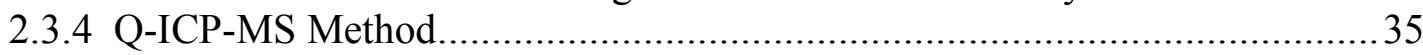

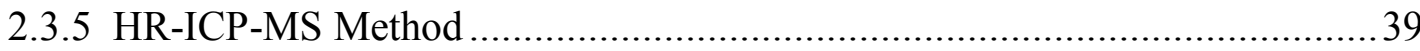

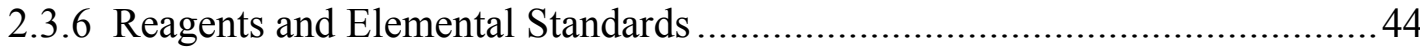

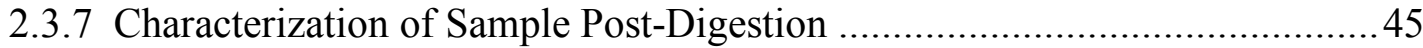

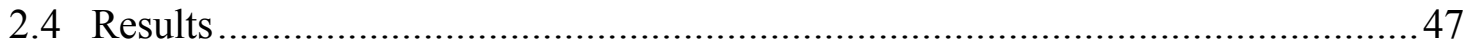

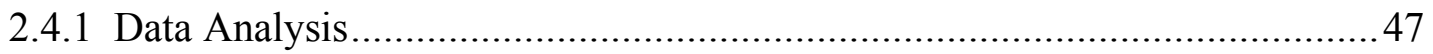

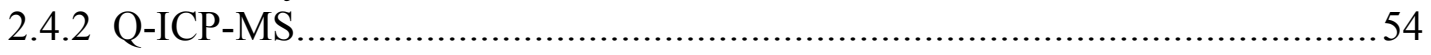

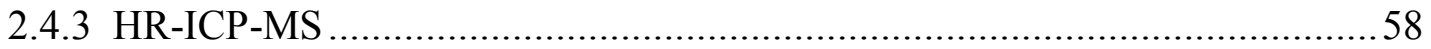

2.4.4 Method Performance with Heroin Samples …………………........................ 61

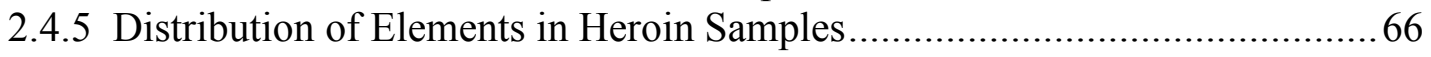

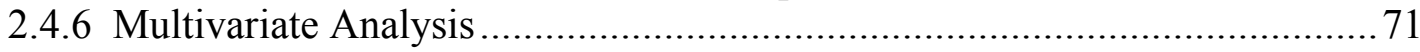

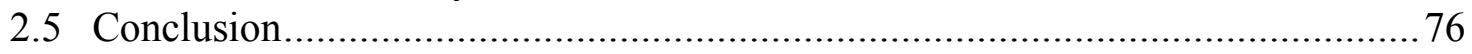

CHAPTER 3. FORENSIC SAMPLE COMPARISON OF HEROIN ….......................... 79

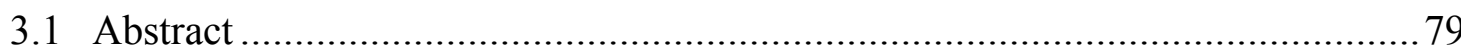

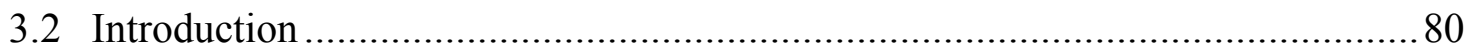

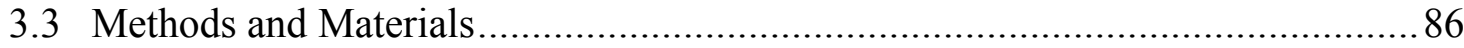

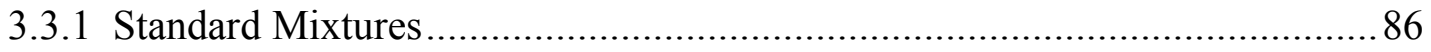

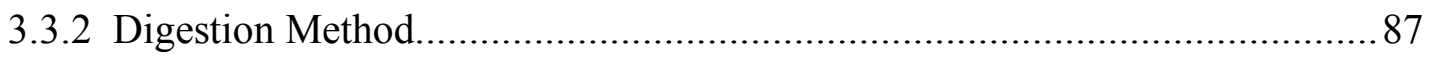




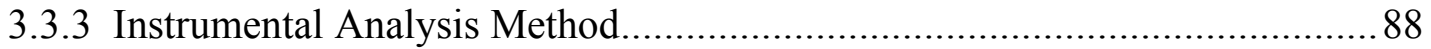

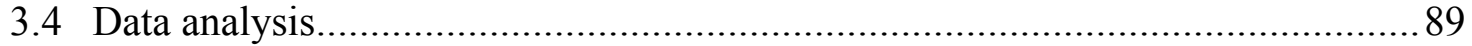

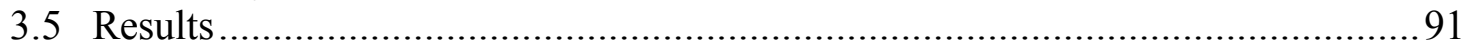

3.5.1 Calculating Error Rates and Random Match Probability...............................91

3.5.2 Matching Simulated Case Samples............................................................ 95

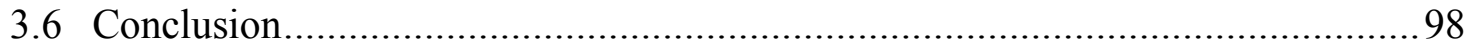

CHAPTER 4. STRONTIUM ISOTOPE RATIO ANALYSIS OF HEROIN ................101

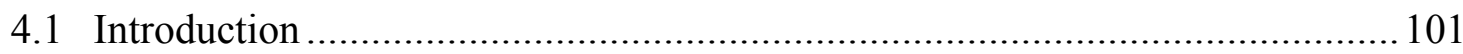

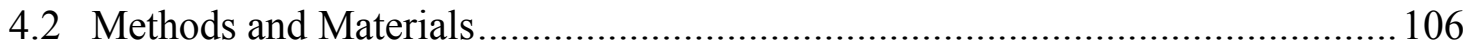

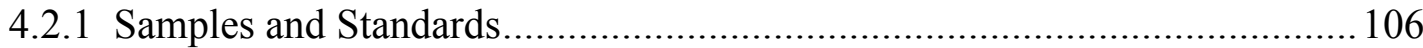

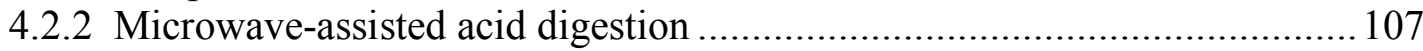

4.2.3 Extraction Chromatography for Strontium Isotope Ratio Analysis.............. 109

4.2.4 High-precision Strontium Isotope Ratio Analysis ....................................... 111

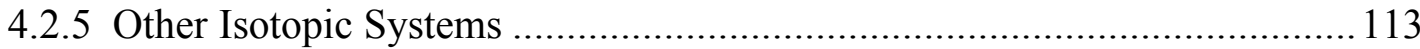

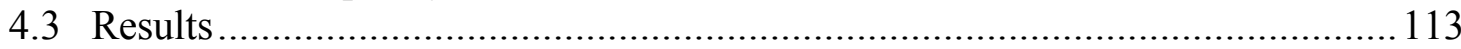

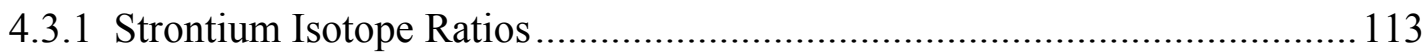

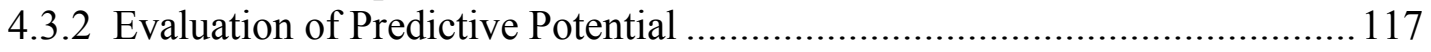

4.4 Merging Elemental Data with ${ }^{87} \mathrm{Sr} /{ }^{86} \mathrm{Sr}$ Isotope Ratios ................................... 119

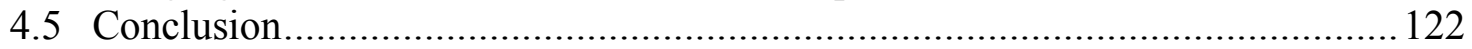

CHAPTER 5. ADDITIONAL ANALYSIS FOR HEROIN PROFILING .....................125

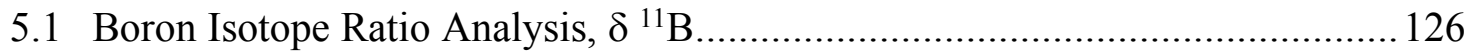

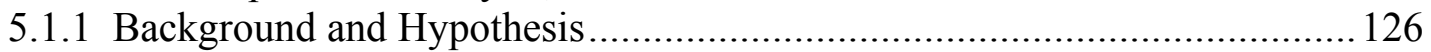

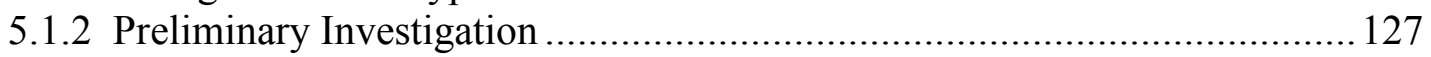

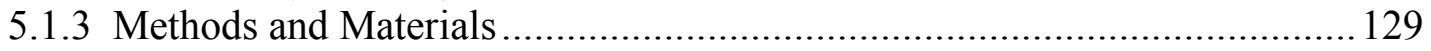

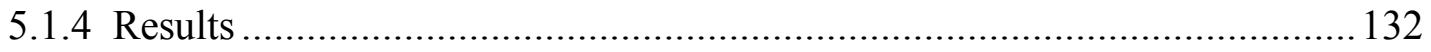

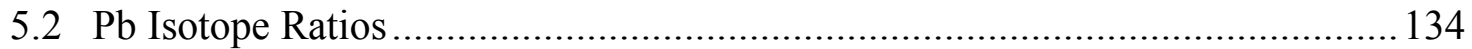

5.3 Assessment of Adulterant Effects.................................................................... 135

5.4 Analysis of Volatile and Semi-Volatile Organic Compounds .......................... 137

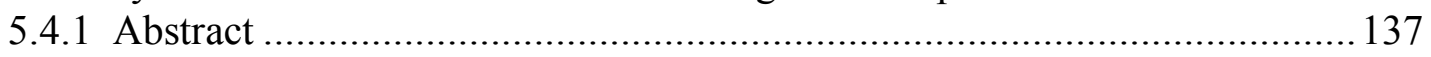

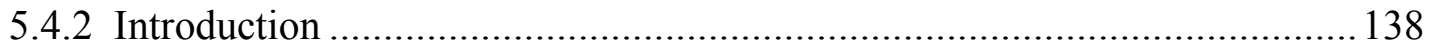

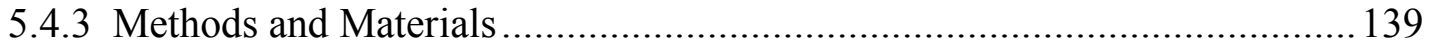

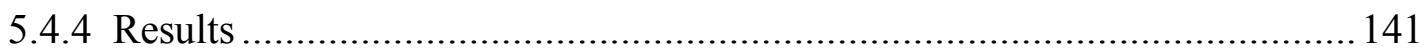

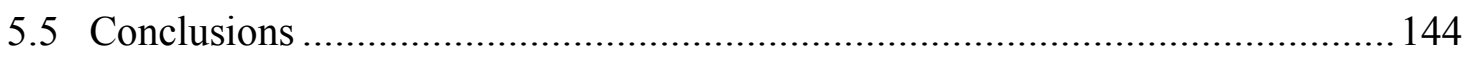

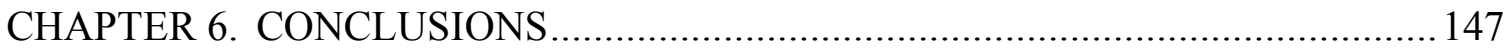

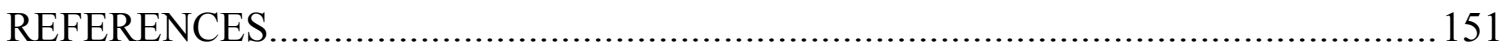

VITA 


\section{LIST OF TABLES}

TABLE

PAGE

Table 1. Design of Plackett-Burman experiment 28

Table 2. Results of significance testing of Plackett-Burman experiment; $t_{\text {effect }}$ values are shown for each parameter. 29

Table 3: Microwave parameters for a batch of 30 heroin samples 34

Table 4: Acquisition parameters for Agilent 7700x method, showing elements in the mode selected in the XR-cell and total integration time/replicate measurement. 37

Table 5: Instrumental parameters for ICP-MS measurements using the Agilent 7700x...38

Table 6. Instrumental parameters for HR-ICP-MS measurements using the Thermo Element 2 .

Table 7. Figures of merit for analysis of the spinach control by Q-ICP-MS $(n=62)$. Missing recovery values indicate elements not reported on the certificate of analysis of NIST SRM 1570a. An asterisk (*) indicates elements were reported only as information values on the certificate of analysis of NIST SRM 1570a.

Table 8. Figures of merit for analysis of the heroin control by Q-ICP-MS $(n=47)$.

Table 9. Figures of merit for analysis of the spinach control by HR-ICP-MS ( $n=61)$. Missing recovery values indicate elements not reported on the certificate of analysis of NIST SRM 1570a. An asterisk (*) indicates elements were reported only as information values on the certificate of analysis of NIST SRM 1570a.

Table 10. Figures of merit for analysis of the heroin control by HR-ICP-MS $(n=48) \ldots . .60$

Table 11. Elements of GFX2-83-8002/9 with Repeatability ( $<30 \%$ RSD) and Reproducibility $\left(>0.7 \mathrm{R}^{2}\right) ; \mathrm{N}=8$.

Table 12. Elements of 88130 with Repeatability ( $<30 \%$ RSD) and Reproducibility $\left(>0.7 \mathrm{R}^{2}\right) ; \mathrm{N}=8$

Table 13. Elements of 88210 with Repeatability ( $<30 \%$ RSD) and Reproducibility $\left(>0.7 \mathrm{R}^{2}\right) ; \mathrm{N}=8$. 
Table 14. Prediction of Heroin Origin by linear discriminant analysis using $\mathrm{LOG}_{10}$ $\left({ }^{23} \mathrm{Na},{ }^{24} \mathrm{Mg},{ }^{52} \mathrm{Cr},{ }^{57} \mathrm{Fe},{ }^{66} \mathrm{Zn},{ }^{90} \mathrm{Zr},{ }^{111} \mathrm{Cd},{ }^{208} \mathrm{~Pb}\right.$ and $\left.{ }^{238} \mathrm{U}\right) \mu \mathrm{g} / \mathrm{g}$ mass concentrations. ..... 74

Table 15. Canonical coefficients of linear discriminant analysis by $\operatorname{LOG}_{10}\left({ }^{23} \mathrm{Na}\right.$,

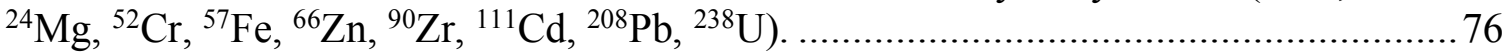

Table 16. Figures of merit for analysis of the spinach control by Q-ICP-MS ( $\mathrm{n}=62)$. Missing recovery values indicate elements not reported on the certificate of analysis of NIST SRM 1570a and an asterisk (*) indicates elements that were reported only as information values.

Table 17. Summary of error rates and calculation of random match probability over a range of match criteria. $\mathrm{ND}=$ not defined

Table 18: Collector configuration for $\mathrm{Sr}$ isotope analysis.

Table 19. Prediction of Heroin Origin by logistic regression of ${ }^{87} \mathrm{Sr} /{ }^{86} \mathrm{Sr}$ isotope ratios 118

Table 20. Prediction of Origin by linear discriminant analysis by $\mathrm{LOG}_{10}\left({ }^{23} \mathrm{Na},{ }^{52} \mathrm{Cr}\right.$, ${ }^{66} \mathrm{Zn},{ }^{90} \mathrm{Zr},{ }^{111} \mathrm{Cd},{ }^{238} \mathrm{U}$ ) and ${ }^{87} \mathrm{Sr} /{ }^{86} \mathrm{Sr}$ isotope ratios

Table 21. Canonical coefficients of linear discriminant analysis by $\mathrm{LOG}_{10}\left({ }^{23} \mathrm{Na},{ }^{52} \mathrm{Cr}\right.$, ${ }^{66} \mathrm{Zn},{ }^{90} \mathrm{Zr},{ }^{111} \mathrm{Cd},{ }^{238} \mathrm{U}$ ) and ${ }^{87} \mathrm{Sr} /{ }^{86} \mathrm{Sr}$ isotope ratios.

Table 22. Prediction of Origin by means comparison of boron $\delta 10 / 11$ values by region

Table 23: Quantiles of distribution of total Pb concentrations $(\mu \mathrm{g} / \mathrm{g})$.

Table 24. Pearson correlation coefficients and linear relationship slope shown for all elements with $\mathrm{R}^{2}>0.7$

Table 25: List of compounds identified in headspace of four unique heroin samples using CMV-GC/MS technique. 


\section{LIST OF FIGURES}

FIGURE

PAGE

Figure 1. Pie chart showing sample description. Samples marked "test" were initially unknown.

Figure 2. The sample introduction system of the Thermo Element 2 HR-ICP-MS.

Figure 3: SRM NIST1570a before digestion (A), an unsuccessful digestion because of excessive microwave power causing sample boiling and loss (B), an unsuccessful digestion because hydrogen peroxide was omitted from digestion vessel bath $(C)$ and a successful digestion of SRM NIST1570a with optic transparency (D).

Figure 4: Digested high-purity morphine (upper row) and heroin (lower row) in concentrated nitric acid; samples were provided by DEA. 26

Figure 5. Thermo Element 2 HR-ICP-MS (left) and Agilent 7700x Q-ICP-MS (right) in the Trace Evidence Analysis Facility (TEAF) at FIU.

Figure 6. Schematic of a Thermo Element 2 HR-ICP-MS with reversed Nier-Johnson geometry. Modified from [48].

Figure 7. Left: moveable slits in the Thermo Element 2 HR-ICP-MS. Right: comparison of the peak shape, resolution, and sensitivity of the three mass resolutions, modified from [48].

Figure 8. Baseline resolution of ${ }^{75} \mathrm{As}^{+}$(left) from the $\left({ }^{40} \mathrm{Ar}^{35} \mathrm{Cl}\right)^{+}$interference (right) in high resolution by the Thermo Fisher Element 2 HR-ICP-MS in a solution of $4 \mathrm{ppb}$ As, $0.08 \mathrm{M} \mathrm{HCl}$ and $0.6 \mathrm{M} \mathrm{HNO}_{3}$

Figure 9. Titration with $\sim 0.1 \mathrm{M} \mathrm{NaOH}$ solution of heroin samples to determine sample mass/post-digest acid molarity. Only one titration was performed for each sample as obtaining the range of nitric acid molarity was more desirable than obtaining a precise determination for each sample.

Figure 10. Levy-Jennings Control chart of ${ }^{43} \mathrm{Ca}$ in SRM NIST 1570a as measured by Q-ICP-MS.

Figure 11. Levy-Jennings Control chart of ${ }^{51} \mathrm{~V}$ in SRM NIST 1570 a as measured by Q-ICP-MS. 
Figure 12. Levy-Jennings Control chart of ${ }^{66} \mathrm{Zn}$ in SRM NIST 1570a as measured by Q-ICP-MS.

Figure 13. Levy-Jennings Control chart of ${ }^{88} \mathrm{Sr}$ in SRM NIST 1570a as measured by Q-ICP-MS.

Figure 14. Levy-Jennings Control chart of ${ }^{238} \mathrm{U}$ in SRM NIST 1570a as measured by Q-ICP-MS.

Figure 15. Levy-Jennings Control chart of ${ }^{43} \mathrm{Ca}$ in GFX2-83-8009/2 as measured by Q-ICP-MS.

Figure 16. Levy-Jennings Control chart of ${ }^{51} \mathrm{~V}$ in GFX2-83-8009/2 as measured by QICP-MS.

Figure 17. Levy-Jennings Control chart of ${ }^{66} \mathrm{Zn}$ in GFX2-83-8009/2 as measured by Q-ICP-MS.......

Figure 18. Levy-Jennings Control chart of ${ }^{88} \mathrm{Sr}$ in GFX2-83-8009/2 as measured by Q-ICP-MS.

Figure 19. Levy-Jennings Control chart of ${ }^{238} \mathrm{U}$ in GFX2-83-8009/2 as measured by Q-ICP-MS.

Figure 20. $\mathrm{x} / \mathrm{y}$ plot of $\mu \mathrm{g} / \mathrm{g}$ concentration heroin samples measured by the Agilent 7700x Q-ICP-MS on the x-axis and the Thermo Fisher Element2 HR-ICP-MS on the $\mathrm{y}$-axis for $\mathrm{Cu}, \mathrm{Zn}, \mathrm{Sr}$ and $\mathrm{Ba}$.

Figure 21. Levy-Jennings Control Chart of 60Ni for SRM NIST 1570a. .56

Figure 22. Log-scale box and whisker plots of concentration (X, $\mu \mathrm{g} / \mathrm{g})$ data obtained on the quadrupole ICP-MS. There are three points of data for each sample heroin. Total lead was determined from the sum of the isotopes ${ }^{206} \mathrm{~Pb},{ }^{207} \mathrm{~Pb}$ and ${ }^{208} \mathrm{~Pb}$.

Figure 23. Box plot of $\log _{10}\left({ }^{23} \mathrm{Na},{ }^{24} \mathrm{Mg},{ }^{52} \mathrm{Cr},{ }^{57} \mathrm{Fe}\right.$ and $\left.{ }^{66} \mathrm{Zn}\right) \mu \mathrm{g} / \mathrm{g}$ separated by region. The boxes represent the $25 \%$ to $75 \%$ quantiles and whiskers are the $5 \%$ to $95 \%$ quantiles; the median (line) and mean are also shown (star). MEX-SA is shown in red with diagonal fill, SA is shown in blue with crossed fill, SEA is shown in gold with a horizontal fill and SWA is shown in green with a checkered fill 
Figure 24. Box plot of $\log _{10}\left({ }^{90} \mathrm{Zr},{ }^{111} \mathrm{Cd},{ }^{208} \mathrm{~Pb}\right.$ and $\left.{ }^{238} \mathrm{U}\right) \mu \mathrm{g} / \mathrm{g}$ concentration by region. The boxes represent the $25 \%$ to $75 \%$ quantiles and whiskers are the $5 \%$ to 95\% quantiles; the median (line) and mean are also shown (star). MEX-SA is shown in red with diagonal fill, SA is shown in blue with crossed fill, SEA is shown in gold with a horizontal fill and SWA is shown in green with a checkered fill

Figure 25. Plot of type 1 and type 2 error rates with increasing coefficient of $s$ used for match criteria. The error rates using match criteria comparing the range of uncertainty of the test set $\left(\mathrm{s}_{1}\right)$ to that of the reference set $\left(\mathrm{s}_{2}\right)$ are shown in black. The error rates using match criteria comparing the mean value of the test set $\left(\mathrm{x}_{1}\right)$ to the uncertainty of the reference set $\left(\mathrm{s}_{2}\right)$ are shown in red.

Figure 26. Heatmap showing samples with $\leq 5$ matching elements in white and 6-10 matching elements in increasingly darker shades of gray. The samples matching at all 11 elements are shown in red with striped fill and generally are found along the diagonal, suggesting similar origin with other members of their test groups. Samples were not compared to themselves.

Figure 27. Hierarchical cluster analysis constellation plot (left) and principle component analysis plot (right) of sample replicates of groups A, B, E, F, G, H, I and K.

Figure 28. Heatmap of test samples (A'-K') compared to the aggregate profiles of a reference group of samples (A-K). Samples with $<5$ matching elements are shown in white and 5-10 matching elements in increasingly darker shades of gray. The samples matching at all 11 elements are shown in red with stripe fill.

Figure 29. Effect of column chemistry (green circles), compared to direct dilution (red diamonds) on the measured values of ${ }^{87} \mathrm{Sr} /{ }^{86} \mathrm{Sr}$ in 12 authentic heroin samples. Samples JD2-62-2A/B and JD2-53-9A/B are pairs of duplicate digestions of two different heroin samples.

Figure 30. Means and uncertainty of ${ }^{87} \mathrm{Sr} /{ }^{86} \mathrm{Sr}$ isotope ratios for powder heroin samples A-D (arbitrary ID assignment), heroin duplicate standard and NIST SRM 1570a, where all samples were separately digested, prepared and analyzed. Error bars are shown for all samples but are too small to be seen for the heroin duplicate standard and for NIST SRM 1570a. $\mathrm{N}=3$ for all samples except for $\mathrm{C}$ (SEA), where $\mathrm{N}=4$.

Figure 31. Box and whisker plot of the distributions and group means of ${ }^{87} \mathrm{Sr} /{ }^{86} \mathrm{Sr}$ isotope ratios for powder heroin 
Figure 32. 3D scatter plot of canonical score of 198 samples for which $\mathrm{LOG}_{10}\left({ }^{23} \mathrm{Na}\right.$, $\left.{ }^{52} \mathrm{Cr},{ }^{66} \mathrm{Zn},{ }^{90} \mathrm{Zr},{ }^{111} \mathrm{Cd},{ }^{238} \mathrm{U}\right)$ and ${ }^{87} \mathrm{Sr} /{ }^{86} \mathrm{Sr}$ data are available. MEX-SA is shown in red, SA in blue, SEA in gold and SWA in green.

Figure 33: Observing fractionation of boron isotopes using microwave assisted-acid digestion (blue dots) as compared to a closed vessel digestion method (PFA; green dots).

Figure 34. Luer lock assembly of boron exchange separation columns, packed with ground Amberlite IRA-743 resin and filtered using Whatman ashless filter paper.

Figure 35. Box plot and data points of boron isotope ratio $\left(\delta^{11} \mathrm{~B}\right)$ measured in heroin samples by region of production. Box plot shows $25 \%$ to $75 \%$ quantile within the boxes and $5 \%$ to $95 \%$ within the whiskers. The median value of each region is indicated by the horizontal line within the boxes and the mean value of the region by the star.....

Figure 36. X/Y plot of measured elemental concentrations ${ }^{23} \mathrm{Na},{ }^{24} \mathrm{Mg},{ }^{208} \mathrm{~Pb}$ and ${ }^{238} \mathrm{U}$ over the fraction of adulterant, 03402 - caffeine, in a mixture with a test heroin sample. The neat heroin sample is at the 0 fraction and the neat cut is at the 1.0 fraction.

Figure 37. Sampling setup for headspace extraction of heroin samples using CMV.

Figure 38: Relative abundance of organic compounds in the headspace of heroin samples by region

Figure 39: Relative abundance of organic compounds in the headspace of heroin samples by region, compounds $\geq 15 \%$ relative abundance not shown. 


\section{CHAPTER 1. INTRODUCTION}

\subsection{Statement of the Problem}

Heroin was discovered and first synthesized in 1874 by Charles Wright in London at St Mary's Hospital Medical School [1]. Heroin was first commercially developed in the late nineteenth century by scientists at Bayer and Co, who also were responsible for the development of aspirin [2]. Initially marketed as a cough suppressant, like the less-potent opiate codeine is today, heroin gained infamy quickly because of its great potential for addiction and was consequently removed from the consumer market. Today heroin is illicitly produced in Mexico, South America, Southeast and Southwest Asia from opium poppies and profits from its distribution and sales are used to finance the operations of criminal and terrorist organizations [3]. Drug smugglers traffic heroin internationally to wealthy countries such as the United States, where demand for the drug is high, to increase profitability.

In the United States the level of abuse of heroin and synthetic opioids is often described in the media as an epidemic, with overdoses of heroin killing more than 15,000 and synthetic opioids killing more than 20,000 individuals in the US in 2016 [4]. The effort to curtail the trafficking and distribution of heroin, which is often a vehicle for synthetic opioids, is of interest to law enforcement and intelligence agencies. Having the capability to chemically analyze heroin samples and statistically associate samples of common origin (as well as discriminate heroin of different origins) is beneficial to building the network of knowledge surrounding the heroin black markets. Being about to predict the origin of 
seized heroin allows law enforcement agencies to apply resources strategically, such that they may have the most beneficial effects on reducing the propagation of heroin.

\subsection{Review of Current Methods for Profiling Heroin}

French forensic scientists first published a review on the various approaches to chemical profiling of heroin in 1997; efforts had been underway for several decades preceding the publication [5]. The authors of the review recognized that no single method was sufficient to capture the total degree of variation among heroin samples, especially considering that heroin producers could change their methods to improve yield or adapt to the availability of reagents. Therefore Besacier et al. generalized the procedure for chemically profiling heroin into three processes, chemical analysis of the opioid compounds, chemical analysis of processing impurities and analysis of stable isotopes [5]. Naturally there are other methods described more recently for the purpose of chemical profiling of heroin that will be discussed within this chapter, but Besacier et al. recognized that a multi-technique strategy was necessary to effectively determine the common origin of heroin samples [5].

A large portion of research into heroin chemical profiling has been performed at The United States Drug Enforcement Administration's Special Testing and Research Lab (DEA-STRL). Therefore, many of the author names will be repeated in the following sections, many of whom have dedicated more than 20 years to furthering the effort. Scientists at DEA-STRL such as John Casale, Ellen Casale, Donald Cooper, Samuel Cooper, Patrick Hayes, Ira Lurie, David Morello, Sini Panicker, and Steven Toske appear as authors on a number of manuscripts related to the endeavor to chemically profile heroin 
that will be discussed in the following chapter. As heroin is and has been a global adversary, forensic chemistry approaches to profile heroin for strategic intelligence has been developed by French [5], German [6] and Australian [7, 8] government laboratories and will be discussed as well.

\subsubsection{Analysis of Organic Opioid Impurities}

By far the most common means of profiling heroin uses the organic characterization and quantification of a sample's organic opioid impurities. While some differentiation of alkaloids is associated with the natural abundance within the varieties of opium poppy, Papaver somniferum, the methods and reagents used in the chemical processing have far greater contribution to observed differences in the profiles of impurities.

Two-dimensional gas chromatography was utilized to perform complex separations on co-eluting opioid compounds by German authors Gröger et al. in 2008 for the purposes of profiling heroin samples [9]. The resulting 2-D data could be plotted in blocks akin to the pixels of an image and processed by pixel-based Fisher analysis [9]. However, the method reported did not conclusively demonstrate that it could be used to successfully profile heroin originating from different geographic regions and was perhaps more appropriate for inter-sample comparison purposes. Unfortunately, the paper did not specify how the samples of heroin differed, nor did they have a test set of samples used to evaluate the performance of the method as a profiling technique.

\subsubsection{Analysis of Basic Organic Impurities}

Building from a method used for analysis of opium samples [10], Lurie et al. in 2004 described a method wherein capillary electrophoresis (CE) enhanced by dynamically 
coating the capillary with cyclodextrins micelles (micellar electrokinetic capillary electrophoresis, MEKC) was used to analyze the basic organic impurities of heroin samples at greater resolution than previously reported by LC and GC methods [11]. Analyses with higher sensitivity and separations with greater theoretical-plate values than previous LC and GC methods were achieved with the use of dimethyl-ß-cyclodextrin or hydroxypropylB-cyclodextrin coated capillary columns as a result of the increased sample loading permitted and the electroosmotic flow of CE [11]. In addition, CE can offer faster sample analysis times and decreased cost of analysis compared to HPLC.

On the basis of over 20 years of research experience $[12,13]$, Lurie and Toske of DEA-STRL reported on Ultra-performance liquid chromatography tandem-mass spectrometry as a method for analysis of basic and neutral opioid impurities for profiling heroin in 2008 [14]. Using a bimodal separation scheme with a first aqueous solution of $\mathrm{pH} 2.2$ (1\% formic acid) and a second of $\mathrm{pH} 10(10 \mathrm{mM}$ ammonium bicarbonate) allowed for many advantages in separation and overall resulted in high selectivity. The acid condition separation was used for analysis of basic impurities, while the basic conditions were used for neutral impurities. Using an increased amount of non-polar solvent during sample loading ( $25 \%$ v $5 \%$ acetonitrile) allowed for simultaneous separation and detection of basic and neutral impurities, albeit at 10-fold reduction in sensitivity compared to the low $\mathrm{pH}$ conditions [14]. Lurie and Toske's manuscript detailed the observation of impurities by UPLC-MS/MS which had not yet been reported, including narceine, reticuline, laudanidine, codamine, cryptopine, laudanosine and some possible isomers of these compounds [14]. 


\subsubsection{Analysis of Acidic Organic Impurities}

Scientists at the Australian Government Analytical Laboratories developed a method of statistical analysis [8] that uses the GC-FID method in use by the United States Drug Enforcement Administration (DEA) at the time to chemically characterize the acidic and neutral impurities of heroin samples to profile them for intelligence purposes. Their sample set was limited to two classes of heroin, samples that were classified as refined Southeast Asia and those that were not [8]. Using a sample set of 54 samples, over 600 compounds were identified as impurities and potential targets for profiling [8]. To make analysis repeatable, the total number of analytes was reduced to less than 300 because of the infrequency of their observation in sample replicates or observation of the compounds in method blank samples [8]. The profiling parameters were developed from both continuous and dichotomized data, wherein data are simplified by treating them categorically as either present/absent, high/low or 1/0 etc. [8]. Logistic regression analysis of dichotomized data with as few as 4 independent variables was shown to correctly predict $>95 \%$ of samples when challenged only with making SEA vs. Non-SEA classifications [8].

With the exception of black tar heroin, conversion of morphine into heroin takes place under basic conditions. Therefore, the majority of organic impurities are also basic. However, the acidic and neutral impurities can also be informative of characteristic production methods, which vary regionally [15]. Chemists with DEA-STRL have further developed their GC-MS method by which acidic and neutral impurities can be analyzed semi-quantitatively via programmed temperature vaporizing injector-gas chromatography mass-spectrometry (PTV-GC-MS) to profile samples from each of the four major regions 
[15]. The analysis was preceded by liquid/liquid extraction and subsequent derivatization by MTSFA, N-Methyl-N-trimethylsilyl-trifluoroacetamide. As one might expect, as refinement of heroin samples increased, the levels of acidic and neutral impurities decreased to sub-ppm levels relative to morphine [15]. The work by Morello et al. yielded a robust chemical profiling method and increased the understanding of the nuanced differences among heroin samples from different regions of heroin production and subclasses existing within each those regions. One limitation of the method by Morello et al. is that it could not clearly distinguish between the most highly refined heroin samples from South America and Southwest Asia as a result of their low abundances of acid and neutral impurities. The aforementioned South American and Southwest Asian highly-refined heroin samples were only dissimilar on the basis of a few unidentified compounds at very low relative abundances.

Capillary electrophoresis has been shown to offer benefits for the analysis of acidic analyte targets for heroin profiling. A modification of the MEKC procedure described by Lurie et al. using alternative buffer reagents (sodium dodecylsulfate and a phosphate-borate buffer), allowed for the acidic, neutral and weakly basic impurities of heroin samples to be separated [11].

\subsubsection{Analysis of Residual Solvents}

Trapped solvents that are occluded within the crystalline matrix of illicit drugs during acidic precipitation can be analyzed for profiling [16-18]. Differences in the mixtures of organic solvents through which $\mathrm{HCl}$ gasses are bubbled or to which liquid $\mathrm{HCl}$ 
is directly spiked, can produce distinct solvent composition within the final $\mathrm{HCl}$ salt product with amine drugs such as cocaine $\mathrm{HCl}$ and heroin $\mathrm{HCl}$ [18].

In 1995, Morello and Meyers reported a static headspace-gas chromatography-mass spectrometry (SHS-GC-MS) method to sample the residual solvents of both heroin $\mathrm{HCl}$ and cocaine $\mathrm{HCl}[18]$. The samples were first dissolved in saturated sodium sulfate solution to release any occluded solvents from the drug's crystalline matrix. Static headspace-gas chromatography-mass spectrometry is performed by sampling a consistent volume of headspace gas above a sample, which can optionally be heated and/or mixed, and directly transferring the sampled headspace gas into the inlet of a GC. The compounds within the headspace are separated on the fused-silica column and qualitatively and quantitatively analyzed by mass spectrometry by means of deuterated internal standards and external calibration solutions. Morello and Meyers also reported testing of common adulterants and did not find that adulterants made a significant contribution to the solvent profile of heroin samples, except in the case of amine drugs in the form of an $\mathrm{HCl}-\mathrm{salt}$, such as diphenhydramine $\mathrm{HCl}[18]$. The most common solvents encountered for heroin samples were ethyl acetate, acetone, ethyl ether, methyl ethyl ketone and toluene [18]. The SHSGC-MS method described by Morello and Meyers shows potential for heroin profiling but has not been published demonstrating its use in such a way.

\subsubsection{Isotope Ratio Analysis}

Stable isotope ratios have been used to profile heroin origins and associate heroin samples to a geographic region of origin. A method using gas chromatography isotoperatio mass spectrometry (GC-IRMS) has been shown to have utility in associating samples 
from each of the four, major regions of heroin production based upon their $\delta^{13} \mathrm{C}$ and $\delta^{15} \mathrm{~N}$ isotopic compositions [19]. A few years later there was a study authored by Casale et al. with DEA-STRL to determine the isotopic fractionation taking place during the chemical refinement of morphine into heroin final product [20].

\subsubsection{Elemental Analysis of Heroin}

The earliest report of heroin profiling by ICP-MS was published in 1998 by Myors et al [7]. Myors et al. proposed criteria for evaluating an element's repeatability and reproducibility, with a cut-off RSD value of $\leq 30 \%$ and a Pearson $R^{2}$ coefficient of $\geq 0.6$ [7]. The repeatability and reproducibility of the method described herein is shown in Chapter 2.3.1. Myors et al. developed an analytical method using inductively coupled plasma-mass spectrometry (ICP-MS) to quantitatively analyze 73 elements to geographically profile heroin [7]. As with the Myors et al. method for profiling with GCMS analysis of organic composition [8], the data set was comprised of continuous as well as dichotomized data used in logistic regression and supported by unsupervised multivariant statistical analysis [7]. Some drawbacks of the results were the classification of heroin samples as either SEA or non-SEA, which is not likely useful to a region where the majority of the heroin originates from the Western Hemisphere, such as the United States. Nor does it make any attempt to distinguish heroin of non-SEA origin. Another point to mention is that only $\mathrm{Na}, \mathrm{Ca}, \mathrm{Zn}$ and $\mathrm{Zr}$ were elements that are deemed informative in both this dissertation and the paper published by Myors et al. [7]; other elements used such as $\mathrm{As}, \mathrm{Ce}, \mathrm{Cs}$ and Gd were often observed in very low concentrations in the samples 
analyzed as part of this dissertation's research and did not meet standards for reproducibility in all of the samples tested, see subchapter 2.4.4.

\subsubsection{Use of Heroin Profiling in Casework}

Profiling techniques not only provide a means for association of samples which are of common origin and have similar production methods, but they also allow for discrimination of unique or novel methods of preparation. John Casale and his coauthors have reported on multiple occasions of how unique methods of heroin preparation can be distinguished from those more commonly encountered in casework [21-23]. In the analysis of samples seized from a North Korean merchant ship and the associated port of call, the authors were able to make discrimination of the samples based upon the accepted methods for heroin signature profiling (at the time) as well as analysis of stable isotope ratios [21, 22]. The authors determined the heroin was of a unique type one the basis of its $\delta^{13} \mathrm{C}$ value being dissimilar to samples originating from Mexico, South America, Southwest Asia and Southeast Asia [21].

Although previously reported with cocaine [24], scientists from DEA-STRL witnessed the controlled processing of heroin by way of an unreported method using bleach, sodium hypochlorite and afterward obtained the sample for chemical analysis [23]. The chemist stated that the purpose of using the bleach was to whiten the heroin product, which usually is reflective of high-quality (refined) heroin product [15]. The procedure using sodium hypochlorite produced chlorinated heroin derivatives which could be incorporated into profile techniques to identify heroin samples following the bleach method. Nine chlorinated opioids, 1-chloroheroin, 1-chloroacetylcodeine, 1-chloro-O6- 
monoacetylmorphine, 2 '-chloropapaverine and five that could not be characterized were observed and studied by GC-MS, LC-MS, ${ }^{1} \mathrm{H}$ NMR and ${ }^{13} \mathrm{C}$ NMR, however the process resulted in almost no heroin remaining in the final product as a result of the excessive amounts of bleach used by the clandestine chemist [23]. Nearly all of the heroin was chlorinated during the oxidation.

\subsection{Conclusion}

With the exception of publications made by researchers from DEA-STRL, most other studies do not consider authentic samples from each of the four, main heroinproducing regions. Some only classify samples a "Type-X" or "Non Type-X." Failing to consider all four regions will produce a method may not be relevant in the dynamic global heroin market. Obviously, the supply and heroin market characteristic in Europe will not be consistent with that of the United States, therefore being able to distinguish between all of the major sources of heroin will be more useful to the various organizations standing in the way of the international heroin cartels.

Many of the methods described for heroin profiling rely upon sophisticated methods of instrumental analysis and often complex statistical means of data interpretation. One limitation to some of the published reports is the inability of the reader to implement the methodology either through incomplete reporting of the explicit parameters used in determining provenance or because full explanation of the statistical approach was taken for granted. This could be as a result of the fact that there is a reasonable measure of security with respect to some methods of analyses, for example, there may have been a desire to conceal intricate details of the method to inhibit counter-measures by the heroin producers. 
In other cases, the parameters used in the profiling method number into the hundreds and a full delineation of each would ruin the brevity of a scientific manuscript.

In any case, it is useful for the continuation of research in the area of illegal drug profiling for authors to fully describe the methodology such that a reader is able to replicate the results of the study and can understand the rationale for the selection of useful parameters. Many analytes are likely redundant or otherwise not informative; describing the process by which useful profiling parameters are chosen, is invaluable. It should be the burden on the authors to fully describe details to prove that the method of profiling is reasonable to the satisfaction of a critical reader, and at the very least provide some discussion as to how the parameters work to provide differences between groups. 


\section{CHAPTER 2. ELEMENTAL ANALYSIS OF HEROIN}

\subsection{Abstract}

Elemental impurities are of interest in the effort to profile heroin, a US Schedule 1 narcotic, and determine a seized sample's region of origin. A profile of elemental impurities is imparted to the heroin sample through cultivation of opium poppy farmland, local water supplies, biological uptake from the environment into the plant, and from human processing of the natural material, morphine, into the heroin final product. Using 223 authentic samples to construct a linear discriminant model on the basis of the abundances of nine elements, $\left({ }^{23} \mathrm{Na},{ }^{24} \mathrm{Mg},{ }^{52} \mathrm{Cr},{ }^{57} \mathrm{Fe},{ }^{66} \mathrm{Zn},{ }^{90} \mathrm{Zr},{ }^{111} \mathrm{Cd},{ }^{208} \mathrm{~Pb}\right.$, and $\left.{ }^{238} \mathrm{U}\right)$, $88 \%$ of a test set of 169 authentic heroin were correctly associated to their region of origin (Mexico, South America, Southeast Asia or Southwest Asia). To the best of the author's knowledge this dissertation and the associated manuscripts report the first analysis of a large-scale heroin profiling study using authentic, seized heroin samples using quantitative, inorganic analysis of elemental composition acquired through both quadrupole inductively coupled plasma-mass spectrometry (ICP-MS) and high-resolution ICP-MS (HR-ICP-MS) for the purpose of differentiating heroin on the basis of its geographic and/or processing origins.

\subsection{Introduction}

The abuse of opiates and synthetic opioids has been at the forefront of media for the past decade because of its detrimental harms on society. Overdoses continue to increase year after year and the effort to fight the trade of heroin and synthetic opioids is a top priority for Federal law enforcement. The ability to correctly predict the geographic origin 
of seized heroin samples is of great concern to investigators in the efforts to address the reemergence of heroin that has occurred in recent years. To gain strategic intelligence used to intercept drug trafficking and distribution, chemical profiling techniques have been developed to determine provenance and gain understanding of the dynamic, illegal drug market.

According to the Center for Disease Control and Prevention (CDC), heroin-related deaths in 2016 were over 15,000, more than 6 times the rate in 2002 [4]. The epidemic levels of heroin use has been linked to stricter enforcement of the non-medical use of prescription opioids and the transition of users to heroin, which can be cheaper, easier to obtain and more potent than prescription painkillers [25]. Additionally, heroin is often a vehicle for extremely potent synthetic opioids, such as fentanyl and fentanyl analogs which have had an even larger death toll than heroin itself since 2016 with more than 20,000 individuals killed in that year [4]. As of 2017, the DEA has formed a signature program for fentanyl and fentanyl analogs to study inter-sample variations akin to that of the Heroin Signature Program, established in 1977 [26-28].

However, the rise in fentanyl and synthetic opioid abuse has not negatively affected heroin markets nor the availability of heroin. Drug dealers often seek to increase their profits by cutting heroin with adulterants such as cheap, nonprescription drugs (caffeine, dextromethorphan, acetaminophen etc.) or inactive diluents, and offset the decreased potency with trace amounts (mg) of fentanyl [29]. According to the 2017 National Drug Threat Assessment published by DEA Strategic Intelligence Section, heroin compared to fentanyl is more widely accessible (49\% survey respondents reporting heroin as highly 
available, compared to $15 \%$ for fentanyl), responsible for a greater share of violent crimes (36.3\% and 5.5\%, respectively) and consumes more of the available law enforcement resources (36.1\% and $8.2 \%$, respectively) [29].

The four geographic regions described 20 years ago are still the major producers of heroin: Mexico, South America, Southeast and Southwest Asia [3]. Forensic drug analysts around the globe continue to gather strategic intelligence on heroin trafficking by conducting profiling studies of seized heroin samples using chemical analysis of its naturally-occurring compounds, processing-related impurities, stable isotope ratios and residual solvent signatures $[6,8,9,11-15,18-20,30-35]$. While attempts to profile heroin using an inorganic analysis of elemental impurities have been investigated [7, 36-39], the method has yet to become adopted for provenance determination, but rather shows promise for conducting street-level sample comparisons [40, 41].

The DEA's interest in determining the utility of elemental analysis in profiling heroin samples, lead to the opportunity to conduct the research described in this dissertation. The specified requirements were investigation of elemental impurities by high-resolution inductively couple plasma-mass spectrometry (HR-ICP-MS). The proposed methods of sample preparation were microwave-assisted acid digestion and analysis of quantitative elemental analysis by ICP-MS and HR-ICP-MS (Chapter 2). Also proposed was isotope ratio analysis by multi-collector inductively coupled plasma-mass spectrometry (MC-ICP-MS) of strontium (Chapter 4), isotope ratio analysis of boron (subchapter 5.1) and isotope ratio analysis of lead (subchapter 5.2). Professor José Almirall at Florida International University was awarded the contract to perform the research, and 
The Scientific Working Group at the US Counter Terrorism Technical Support Office was responsible for management of the contract and funding under award IS-FI-4174.

Budic and Klemenc reported the first instance of microwave-assisted acid digestion for the analysis of major crustal elements in heroin by inductively coupled plasma-atomic emission spectrometry (ICP-AES) [37]. While they were not reporting the method for profiling, Budic and Klemenc measured several elements which were identified as useful in the results for forensic sample comparisons (Chapter 3). The authors reported goodquality digestions with low elemental background, however they observed challenges associated with increased detection limits because of the dilution steps in microwave digestion when using a pneumatic nebulizer with ICP-AES; they overcame this difficulty by using a ultra-sonic (desolvation) nebulizer [37]. The difficulty associated with dilution was overcome in this research of this dissertation by using ICP-MS for elemental quantitative analysis, which has lower detection limits compared to ICP-AES.

Bora et al. reported using only high-purity nitric acid for successful digestions in an earlier-model Milestone digestion oven, using $3 \mathrm{~mL}$ of acid to digest between 200 and $400 \mathrm{~g}$ of heroin in PFA vessels [36]. The oven program was quite simple; it was set to apply $650 \mathrm{~W}$ of microwave power for 20 minutes [36]. From the reported success of this methodology, it was expected that successful digestion of up to $150 \mathrm{mg}$ could readily be achieved using $2.3 \mathrm{~mL}$ of nitric acid in low-volume quartz micro-vessels for lower memory effects compared to using PFA. Repeatability of various acid volumes and oven programs were tested (Section 2.2.2) before establishing the final method parameters. 
Chan et al. reported a validated method of analysis for elemental impurities of heroin by ICP-MS, however their method only used physical and ultrasonic dissolution with $1 \% \mathrm{HNO}_{3}$ [42]. It is very likely that filtration of those samples would be necessary, and digestion would be incomplete under the specified parameters. However, the results of this dissertation chapter were consistent with those reported and similar good repeatability and reproducibility was observed for the elements in common.

\subsubsection{Description of Heroin Samples}

The total number of heroin samples received from (DEA-STRL) was 415 individual powdered heroin samples. The set of samples included one sample each of high purity heroin and morphine for initial testing and sample preparation method development, "authentic" heroin samples of known origin, blind samples of known origin (unknown to the author), truly unknown samples and samples for cutting experiments. The samples had known origins assigned by the signature determination methods at DEA-STRL Heroin Signature Program (HSP). The truly unknown samples were those which did not have conclusive results from the signature analysis methods used by DEA-STRL.

The collection of heroin samples consisted of 54 samples of heroin from Mexico that were manufactured using similar processing methods to those used by clandestine processors in South America (MEX-SA), 98 samples of South American heroin (SA), 36 samples of heroin from Southeast Asia (SEA) and 34 samples from Southwest Asia (SWA). The total number of samples received with known origins was 223 samples. An additional SWA sample with a large sample mass was used as a "Heroin Control" to test the daily repeatability of the methods. To test the statistical provenance prediction methods 
described in this report, 169 "blind" samples were received, the origin of which was initially unknown to the researchers at Florida International University (FIU). Additionally, eight samples of truly unknown origin were received. Lastly, 13 samples consisting of authentic seized adulterants and a mixture of the adulterants with a heroin sample were received and analyzed to assess the contribution of adulterants and diluents to the profile of inorganic elements. The sample summary is shown on Figure 1.

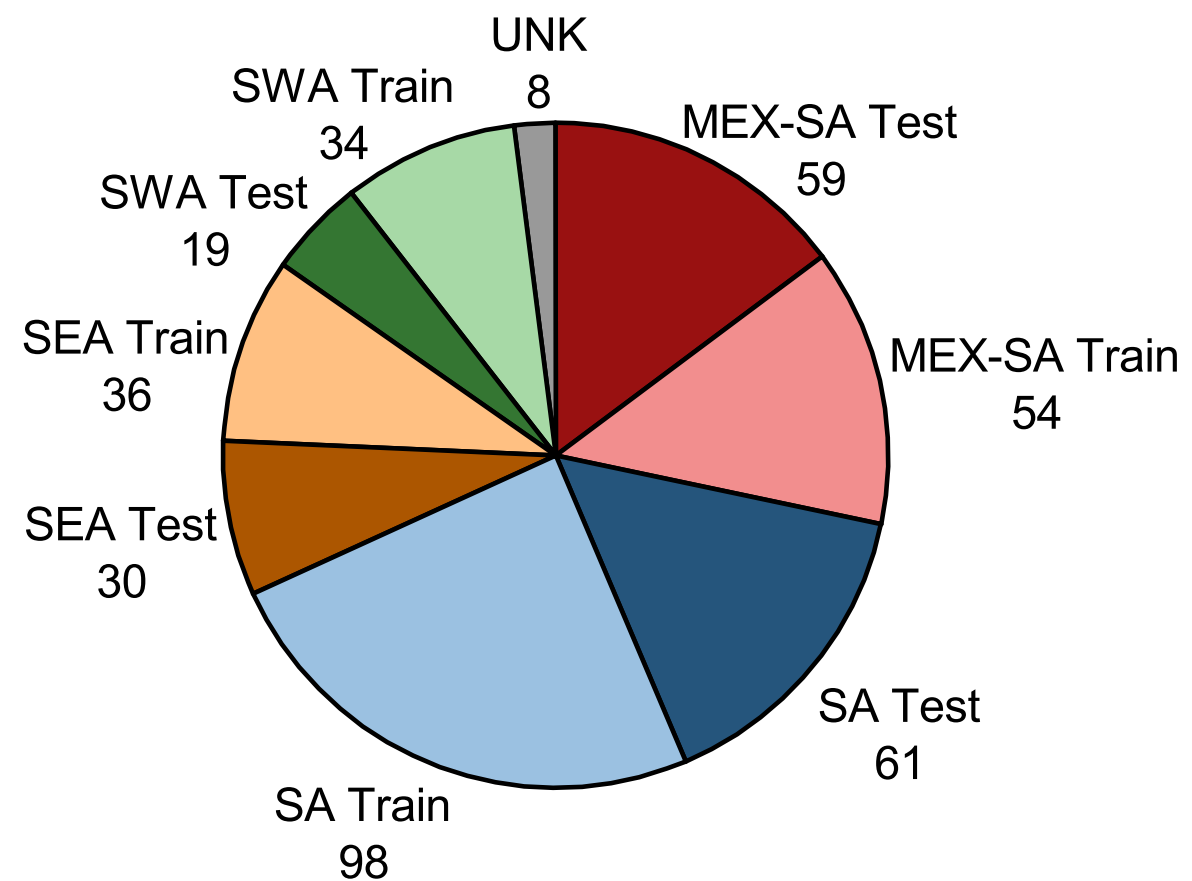

Figure 1. Pie chart showing sample description. Samples marked "test" were initially unknown.

Representatives of the HSP at the DEA-STRL selected the samples to obtain the most useful information using the potential efficacy of inorganic analysis at supplementing their present provenance determination methods, some of which are summarized in Chapter 1 . In addition to the samples provided for provenance determination, 13 samples consisting of authentic seized cutting agents and mixtures of the cutting agents with a 
heroin sample were received and analyzed to determine the contribution of cutting agents to the profile of inorganic elements, the results of which are described in subchapter 5.3.

\subsubsection{Inductively Coupled Plasma-Mass Spectrometry}

The principle of inductively coupled plasma-mass spectrometry (ICP-MS) centers around the atomization and ionization of the sample in a plasma under an intense, radiofrequency-induced field. The ions of the sample are carried by a gas (generally high purity Argon) through the mass spectrometer interface under high vacuum $\left(>10^{-7}\right.$ Torr). The ions are then directed through the ion optics by charged lenses and then separated by their mass-to-charge ratio $(\mathrm{m} / \mathrm{z})$ by a mass analyzer. Finally, the ions are typically detected by an electron multiplier, which is a discrete dynode array type of ion counter; in the case of the multi-collector ICP-MS, the detector is an array of Faraday cups that directly measure voltage but also features a dynode array for ion counting. The ICP-MS instrument includes the following basic components: sample introduction system, torch, interface, ion optics, mass separator, and ion detector. The ICP-MS, a 7700x (Agilent Technologies, USA) and the HR-ICP-MS, an Element 2 (Thermo Fisher Scientific, USA), which were used in the element quantitation work of this dissertation, operate very similarly, in principle. However, each have unique design features that lend each instrument certain advantages over the other.

The sample introduction systems (see Figure 2) of the instruments used in this work consist of an autosampler to move the sample probe to the specified sample vial, a peristaltic pump (Q-ICP-MS and HR-ICP-MS only) to pump a steady controlled flow of solution through the tubing, an internal standard mixing tee (Q-ICP-MS and HR-ICP-MS 
only) to mix the internal standard with the sample solution, a nebulizer where the solution is sprayed into a fine aerosol using a flow of argon gas, and a spray chamber where the finest and most homogeneous part of the aerosol is directed to the plasma torch. The main differences in the instruments' sample introduction systems is that the 7700x has a Peltiercooled, double pass spray chamber and the Element 2 has a cyclonic spray chamber that is operated at room temperature.

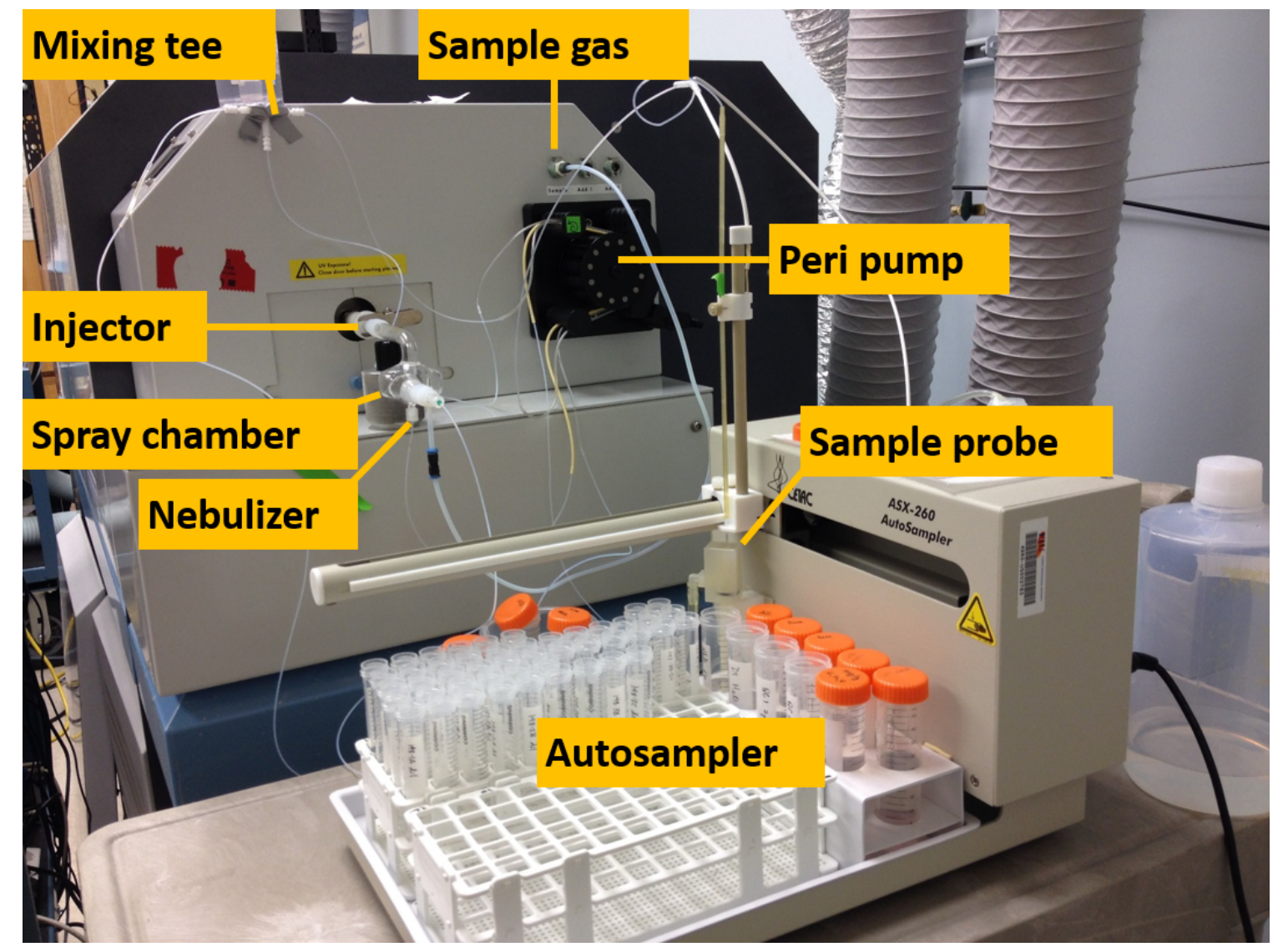

Figure 2. The sample introduction system of the Thermo Element 2 HR-ICP-MS.

The torch is made of concentric tubes of quartz that are open at the end, with the injector in the center. Argon gas flows through the torch between the tubes to stabilize and isolate the plasma from the outer tubes and to carry the sample through the injector into the 
plasma. The torch is surrounded by a radiofrequency (RF) coil that supplies an alternating current with enough power to create an alternating magnetic field. A high voltage spark generates free electrons that are accelerated by the field until some have enough energy to ionize some of the argon atoms, whose electrons in turn ionize other argon atoms in a cascade, creating a plasma [43]. The RF power sustains the hot plasma $(6,000$ to 10,000 $\mathrm{K})$, which is used as an ionization source. In the plasma the sample is vaporized, molecules are broken down into atoms, and positively charged ions are formed at atmospheric pressure.

The next feature of the instrument is the interface region is where the sample ions are transferred from atmospheric pressure to a region that is kept under vacuum to prevent contamination from air and stray ions that could collide with the sample ions. Small diameter orifices of the skimmer and sampler cones provide the passage of ion into the low-pressure vacuum manifold. The central, core channel (z-axis) of the plasma is usually aligned with the orifices of the skimmer and sampler cones for the highest transmittance of ions in to interface region. The gas flows and position of the torch, as well as the RF power, are optimized for maximum signal intensity and signal stability, and minimal formation of oxides and doubly-charged species that could interfere with the ions of interest.

The ion optics are a series of mostly negatively-charged lenses used to accelerate, shape, and direct the beam of sample ions toward the mass analyzer. The mass analyzer separates the ions on the basis of their $\mathrm{m} / \mathrm{z}$. The instruments described in this dissertation differ in the design of their mass analyzers, which are designed to suit their preferred application(s). The Agilent 7700x uses a quadrupole (Q-ICP-MS) mass analyzer for rapid 
analysis and high-sample throughput, and the Thermo Fisher Element 2 uses a doublefocusing magnetic-electrostatic sector (HR-ICP-MS) for high sensitivity and mass resolution. The Thermo Fisher Neptune multi-collector (MC-ICP-MS, discussed in Chapter 4), also uses a double-focusing mass analyzer but in the opposite orientation of electrostatic-magnetic sectors. The ions that make it through the mass analyzer are then detected by the detector (or array of detectors in MC-ICP-MS) where a signal is generated with an intensity proportional to the number of ions (which is related to the concentration of the sample).

One of the drawbacks of ICP-MS analysis is that it is one-dimensional and has no inherent means of separation. All sample elements and polyatomic molecules, both intrinsic and extraneous, are analyzed by mass/charge $(\mathrm{m} / \mathrm{z})$. The main sources of interference are differing species of equal $\mathrm{m} / \mathrm{z}$ that are referred to as isobaric and polyatomic interferences. Although the term isobaric implies equal mass, isobaric (and polyatomic) interferences are of equal $\mathrm{m} / \mathrm{z}$ and not necessarily equal in mass. For example, ${ }^{142} \mathrm{Ce}^{+}$and ${ }^{142} \mathrm{Nd}^{+}$actually do have the same nominal mass (142 u). In very hot regions occurring near the outside edges of the plasma, a second electron can be removed, resulting in doubly-charged species $\left(\mathrm{M}^{2+}\right)$ with a $\mathrm{m} / \mathrm{z}$ of half the normal nominal mass, such as in the example of ${ }^{138} \mathrm{Ba}^{2+}$ and ${ }^{69} \mathrm{Ga}^{+}$[44]. Doubly charged ${ }^{138} \mathrm{Ba}^{2+}$ has a $\mathrm{m} / \mathrm{z}$ of 69 , which is the same $\mathrm{m} / \mathrm{z}$ as singly charged ${ }^{69} \mathrm{Ga}^{+}$. Not only do the polyatomic and doubly-charged species cause interferences at other $\mathrm{m} / \mathrm{z}$, but their formation also reduces the intensity measured at the target isotope's nominal mass. Not surprisingly, the more ${ }^{238} \mathrm{U}^{16} \mathrm{O}^{+}(\mathrm{m} / \mathrm{z}=$ 254) or ${ }^{138} \mathrm{Ba}^{2+}(\mathrm{m} / \mathrm{z}=69)$ that forms, the less there is available of ${ }^{238} \mathrm{U}^{+}$or ${ }^{138} \mathrm{Ba}^{+}$to be measured. 
Polyatomic species can also have the same nominal mass as an isotope of interest. For example, both ${ }^{40} \mathrm{Ar}^{16} \mathrm{O}^{+}$and ${ }^{56} \mathrm{Fe}^{+}$have a nominal mass of $56 \mathrm{u}$ (and also equal $\mathrm{m} / \mathrm{z}$ ). These form by recombination of ions in the cooler regions of the plasma and most commonly include oxides $(\mathrm{M}+16)$, hydrides $(\mathrm{M}+1)$, carbides $(\mathrm{M}+12)$, chlorides $(\mathrm{M}+35)$, and dimers $(2 \mathrm{M})$, where $\mathrm{M}$ is the $\mathrm{m} / \mathrm{z}$ of the single target ion. Often polyatomic isobaric interferences are generated from the matrices of the plasma gas, atmospheric gas, the water and acids used in the solutions, and from the sample matrix itself.

Isotopes without any interferences should be selected for measurement, but sometimes it is not possible because alternative isotopes either have interferences themselves or exist at such low relative abundances that makes them undesirable targets for analysis. Each instrument has its own strategically designed means for separating and detecting ions, enhancing signal and reducing interferences, which will be discussed in subchapters 2.3.4 and 2.3.5.

\subsection{Methods and Materials}

\subsubsection{Method Development}

In 2012 Chan and Wong published on validating a method for the use of ICP-MS with respect to heroin analysis [42]. However, during to the course of the research described in this dissertation, an apparent flaw was noted in their reported sample preparation. It was noted that described method of sample dissolution was likely not sufficiently aggressive for total digestion of the heroin. During the course of method development, it was observed that there was incomplete dissolution of heroin samples in closed perfluoroalkoxy alkane (PFA) vessels under conditions of 6 M Nitric Acid and 
heated to near boiling. Filtering away residual solids, as Chan and Wong report should not be considered a quantitative extraction method; in addition, there was no certified reference material used to evaluate the completeness of trace element liberation from the matrix [42]. Spiking a mixture of elements in acidic solution onto a sample cannot be expected to produce a realistic representation of the recovery of an extraction method as the elements are already in solution. Therefore, the sample preparation developed for the method described in this chapter followed methods of total sample dissolution by microwaveassisted acid digestion and validated using SRM NIST 1570a, trace elements in spinach leaves [45].

During the process of method development, it was first desired to determine a sample preparation method that would achieve a completely, optically-transparent digestion product. The development of the digestion method, the selection of instrumental parameters and robustness testing experiments were performed with Standard Reference Material (SRM) NIST 1570a, trace elements in spinach leaves. The choice of SRM NIST 1570a for use in method development was for several reasons. The primary reason being that the SRM was certified for its trace element composition by multiple individual laboratories. A second reason was that the sample mass was abundant, while the heroin samples had limited sample mass available to be used for method development. Finally, over the course of some preliminary digestion experiments, it became apparent that producing an optically transparent digestion product with the plant material was more challenging than for powdered heroin samples. 
From the standpoint of producing a robust method, it seemed logical to select a more challenging sample matrix to use during method development. If the method was sufficient for digestion of a more challenging sample matrix, differences in heroin sample matrices would have less of an effect on the digestion efficiency. Despite the fact that heroin samples provided for this work by DEA were not heavily adulterated, "streetquality" samples, there were differences in the opiate composition, the organic impurities and the overall quality of refinement. However, the differences are at the level of the small molecule composition, while the plant material of the SRM 1570a contains pectin, cellulose and other complex cellular macromolecules, which are greater obstacles to achieving complete oxidation. It was expected that a sample preparation method that was suitable to completely digest the complex matrix of SRM NIST1570a would be more than aggressive enough for heroin samples. Therefore, complete digestion would be expected despite relatively minor sample-to-sample differences in the heroin.

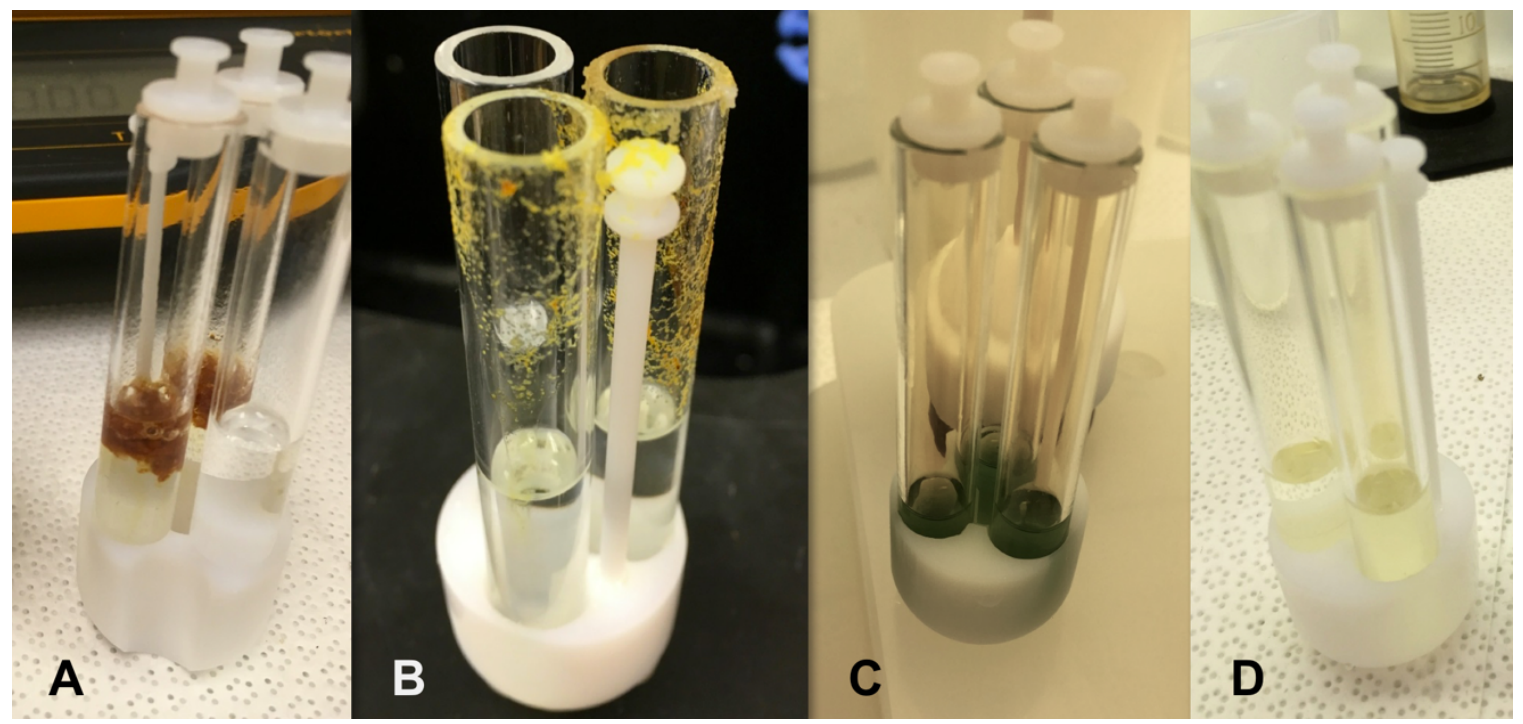

Figure 3: SRM NIST1570a before digestion (A), an unsuccessful digestion because of excessive microwave power causing sample boiling and loss (B), an unsuccessful digestion because hydrogen peroxide was omitted from digestion vessel bath (C) and a successful digestion of SRM NIST1570a with optic transparency (D). 
Nitric acid, $\mathrm{HNO}_{3}$, was initially chosen as the digestion solvent because it is a strong oxidizing agent and will readily oxidize carbon bonds in organic molecules [45]. Addition of hydrogen peroxide $\left(\mathrm{H}_{2} \mathrm{O}_{2}\right)$ and/or water $\left(\mathrm{H}_{2} \mathrm{O}\right)$ can increase the oxidation potential and reduce molarity of nitric acid $\left(\mathrm{HNO}_{3}\right)$. After testing a variety of acid combinations, the choice of Optima-grade $\mathrm{HNO}_{3}$ (Fisher Scientific International Inc., USA) was made because it was the simplest formula that achieved total sample solution resulting in the optically transparent product, which was free of any fine particles. The inclusion of any additional reagents in the mixture, even Ultra-pure Millipore water, not only increased the uncertainty of a successful digestion but also raised the background concentration of ultra-trace elements. Inclusion of other solvents, especially hydrochloric acid $(\mathrm{HCl})$ and hydrogen peroxide $\left(\mathrm{H}_{2} \mathrm{O}_{2}\right)$ produced vapors from the digestion solution. It was impossible to contain solutions of $\mathrm{H}_{2} \mathrm{O}_{2}+\mathrm{HNO}_{3}$ and solution escaped from the digestion vessel before the microwave program could be initiated.

Concurrently with testing of the digestion mixtures, the microwave parameters were adjusted until a complete digestion could be achieved. The Milestone digestion oven, model ETHOS UP, was preprogrammed with methods designed for a variety of sample types. Taking input from Milestone technicians, the maximum microwave power was adjusted depending upon the loading of vessels within the oven. For example, when the oven was loaded with only four (out of a maximum of 15 vessels) the maximum microwave power applied was set to $600 \mathrm{~W}$. During a routine run, when the oven was loaded with 10 vessels, the maximum microwave power was set to $1600 \mathrm{~W}$. The power adjustment was an important modification to the method because the microwave adjusts power output at a rate of $1 \mathrm{~Hz}$, if the temperature in the reference vessel is below the current set-point, the 
microwave applies microwave radiation (generally at the maximum power setting, which at default is $1800 \mathrm{~W})$. For a partially-loaded microwave digestion oven, excessive microwave radiation applied to the sample could produce over heating without sufficient pressure to keep the acid sub-boiling. Therefore, a milder application of microwave energy was desirable to keep from over-heating the solution within the vessel.

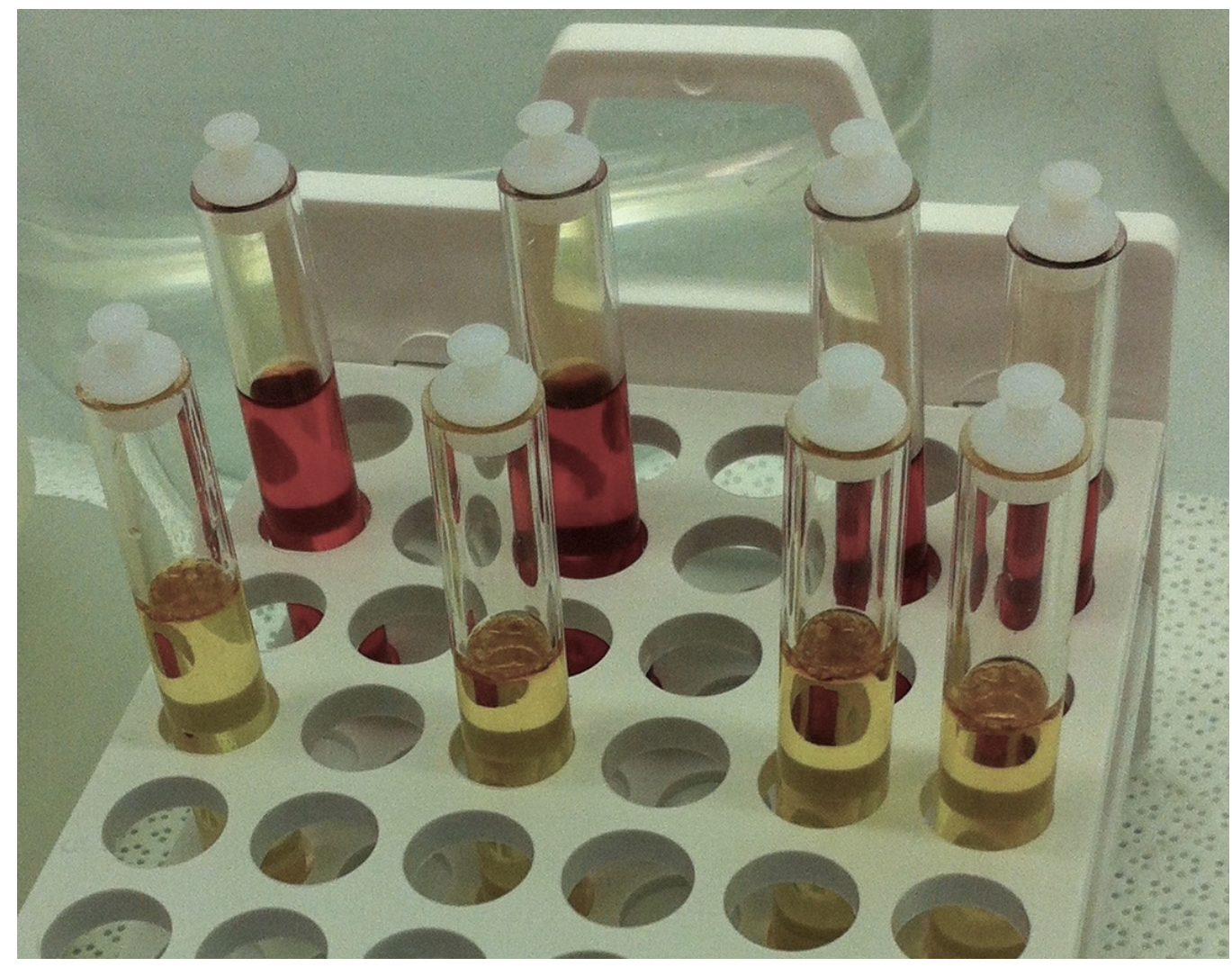

Figure 4: Digested high-purity morphine (upper row) and heroin (lower row) in concentrated nitric acid; samples were provided by DEA.

To determine the source of elements inherent to heroin of the highest-order of refinement, laboratory grade morphine and heroin was provided by DEA. Four replicate samples each of heroin and morphine and four reagent blank samples were placed into micro-insert quartz vessels. Each of four polyether ether ketone (PEEK) digestion vessels 
contains one micro-insert of morphine, one of heroin and one blank. The morphine samples had masses between ranging from 42.4 to $80.8 \mathrm{mg}$ and the heroin samples had masses ranging from 50.5 to $65.0 \mathrm{mg}$. Both of the high-purity heroin and morphine dissolved readily into concentrated nitric acid, producing vibrant colors along with their conventional expected coloration in forensic color testing without any visible particulates remaining (see Figure 4). The morphine samples and heroin samples had very vibrant crimson and gold colorations when dissolved in nitric acid, respectively.

The high-purity morphine and heroin samples were dissolved in the Milestone Ethos UP digestion oven with parameters very near to the final method parameters given on Table 3, however a lower maximum microwave power setting was used as previously mentioned. Following digestion there was no visible particles and all color was gone, leaving the solutions completely transparent. Relative to the SRM NIST 1570a, trace elements in spinach leaves, the high-purity morphine and heroin samples dissolved much more readily in the concentrated nitric acid.

\subsubsection{Testing for Method Robustness}

When both the digestion solution and microwave method were established, slight variations were made to the parameters to determine the robustness of the method. In other words, how much could one deviate from the prescribed acid mixture and microwave settings and still achieve statistically equivalent digestion results. A Plackett-Burman experiment was performed according to ASTM E1319.02 [46] (it has since been updated), by measuring several elemental concentrations in the digestion solutions that were produced after slightly modifying method parameters between each experiment. The 
results of the robustness test provide an assessment of the significance of effects during a multifactorial design of experiment.

The purpose of the Plackett-Burman design of experiment is to reduce the number of trials needed to assess the robustness of a method. For example, to test all possible combinations of five parameters with a high and low setting, one would need to perform $32\left(2^{5}\right)$ separate experiments. With the Plackett-Burman design, various combinations of the high and low parameters will be made to reduce the labor in method development. Of course, there will be some compounding effects by changing multiple parameters between each experiment, which is why is useful to have performed some method optimization beforehand. The Plackett-Burman design of experiment summary can be found below in Table 1, which shows the combinations of high and low method parameters.

Table 1. Design of Plackett-Burman experiment

\begin{tabular}{|crrrrrrrr|}
\hline \hline & \multicolumn{7}{c|}{ Experiment \# } \\
\cline { 2 - 8 } Effect & \multicolumn{1}{c|}{1} & \multicolumn{1}{c|}{3} & \multicolumn{1}{c|}{5} & \multicolumn{1}{c|}{5} & 7 & 8 \\
\cline { 2 - 8 } sample mg & $40-60$ & $20-39.9$ & $20-39.9$ & $40-60$ & $20-39.9$ & $40-60$ & $40-60$ & $20-39.9$ \\
acid volume & $3 \mathrm{~mL}$ & $3 \mathrm{~mL}$ & $2 \mathrm{~mL}$ & $2 \mathrm{~mL}$ & $3 \mathrm{~mL}$ & $2 \mathrm{~mL}$ & $3 \mathrm{~mL}$ & $2 \mathrm{~mL}$ \\
max temp & $220^{\circ} \mathrm{C}$ & $220^{\circ} \mathrm{C}$ & $220^{\circ} \mathrm{C}$ & $200^{\circ} \mathrm{C}$ & $200^{\circ} \mathrm{C}$ & $220^{\circ} \mathrm{C}$ & $200^{\circ} \mathrm{C}$ & $200^{\circ} \mathrm{C}$ \\
ramp time & $10 \mathrm{~min}$ & $20 \mathrm{~min}$ & $20 \mathrm{~min}$ & $20 \mathrm{~min}$ & $10 \mathrm{~min}$ & $10 \mathrm{~min}$ & $20 \mathrm{~min}$ & $10 \mathrm{~min}$ \\
hold time & $20 \mathrm{~min}$ & $10 \mathrm{~min}$ & $20 \mathrm{~min}$ & $20 \mathrm{~min}$ & $20 \mathrm{~min}$ & $10 \mathrm{~min}$ & $10 \mathrm{~min}$ & $10 \mathrm{~min}$ \\
\hline
\end{tabular}

The results of the Plackett-Burman shown on Table 2 indicate very little significance in the effects (when $t_{\text {effect }}$ is greater than 2.37) when changing the tested parameters from their high or low values. An extraction method that uses all of the parameters at the low setting, all at the high setting, any combination of high and low or any setting in between should produce not produce significant differences in overall performance. Nickel did show a slightly higher $t_{\text {effect }}$ value over the threshold value for the 
hold time parameter, however there was some difficulty with repeatability of the ${ }^{60} \mathrm{Ni}$ measurements for SRM NIST 1570a as can be seen in the instrumental validation results in sections 2.4.2 and 2.4.3. Additionally, the hold time parameter had very little effect on any of the other isotopes being monitored. Consequently, it was concluded that the result was anomalous, and the method was robust when performed within the parameter specifications set in the experiment.

\begin{tabular}{|c|c|c|c|c|c|}
\hline \multirow[b]{2}{*}{ Isotope } & \multicolumn{5}{|c|}{ Effect is significant if teffect $>2.35$} \\
\hline & sample mass & acid volume & $\max$ temp & ramp time & hold time \\
\hline${ }^{11} \mathrm{~B}$ & 0.71 & 0.17 & 0.93 & 1.44 & 1.09 \\
\hline${ }^{51} \mathrm{~V}$ & 1.05 & 1.25 & 0.29 & 0.40 & 0.83 \\
\hline${ }^{55} \mathrm{Mn}$ & 0.21 & 0.96 & 1.70 & 0.45 & 0.89 \\
\hline${ }^{59} \mathrm{Co}$ & 1.77 & 0.12 & 1.65 & 0.87 & 0.14 \\
\hline${ }^{60} \mathrm{Ni}$ & 0.85 & 0.61 & 0.28 & 0.33 & 2.39 \\
\hline${ }^{63} \mathrm{Cu}$ & 0.58 & 1.41 & 0.58 & 1.11 & 1.29 \\
\hline${ }^{66} \mathrm{Zn}$ & 1.87 & 1.50 & 0.58 & 0.79 & 0.16 \\
\hline${ }^{85} \mathrm{Rb}$ & 1.21 & 0.68 & 1.12 & 0.73 & 1.26 \\
\hline${ }^{88} \mathrm{Sr}$ & 1.42 & 0.43 & 1.28 & 0.63 & 1.40 \\
\hline${ }^{111} \mathrm{Cd}$ & 0.00 & 0.10 & 0.90 & 1.35 & 0.81 \\
\hline${ }^{208} \mathrm{~Pb}$ & 1.94 & 1.16 & 0.41 & 0.81 & 0.44 \\
\hline${ }^{238} \mathrm{U}$ & 2.03 & 0.60 & 1.22 & 0.46 & 0.23 \\
\hline
\end{tabular}

\subsubsection{Micro-wave Assisted Acid Digestion for Elemental Analysis}

The Environmental Protection Agency's (EPA) Method 3052 for total sample dissolution recommends sample preparation by microwave-assisted acid digestion. The dissolution of heroin samples by microwave-assisted acid digestion was chosen as it could deliver total digestion of organic compounds and minerals which may have been encountered in the sample set of heroin samples. The samples were prepared in such a way that a single acid digestion of the sample could be suitable for all of three methods of 
inorganic analysis (Q-ICP-MS, HR-ICP-MS, and MC-ICP-MS). Digested samples were stored in sealed $15 \mathrm{~mL}$ centrifuge tubes at $4{ }^{\circ} \mathrm{C}$ until instrumental analysis was performed. Samples were not diluted until just prior to instrumental analysis.

Microwave-assisted digestion greatly facilitated the process of sample preparation, making complete digestions much more readily than closed-vessel, hotplate digestion allowing for higher throughput of heroin samples. The heroin samples were prepared in nitric acid with a method modified from the procedures of EPA Method 3052 [45]. The digestion was overly strong for the heroin sample, because the method was optimized for a more challenging matrix as mentioned in subchapter 2.3.1. Because of the complex nature of the plant material compounds and composition of large, carbon-bearing molecules (e.g., peptides, saccharides, cellulose, etc.), the digestions were observed to be much more challenging than for equivalent masses of heroin. The choice of SRM NIST 1570a (trace elements in spinach leaves) as a standard by which to evaluate its digestive power meant the method could be certain to ensure total digestion of heroin samples, which consisted of much smaller molecules.

To oxidize the covalent bonds of the organic heroin matrix, an efficient and reproducible digestion method was most desirable for processing a large number of samples for ICP-MS analysis. Microwave-assisted acid digestion was desirable for achieving total sample decomposition in accordance to EPA method 3052, which defines the parameters necessary to achieve total digestion of organic and soil matrices [9]. The Milestone Ethos-UP microwave digestion oven conforms or greatly exceeds all parameters for EPA method 3052. The rotor and vessels with the greatest pressure tolerance and widest 
application flexibility, the Milestone SK-15 rotor, was chosen to accompany the microwave. Optional $4 \mathrm{~mL}$ quartz micro-insert vials were purchased (as HF was not required for digestion of heroin or standard matrices) that permitted sample and acid volumes to be scaled back compared to most 3052 methods by approximately $70 \%$. The quartz micro-insert vials also allowed three replicates to be digested simultaneously, which increased the throughput of the 10 vessels to 30 samples per digestion. All digestion vessels and accessories were purchased from Milestone, Scientific (USA).

Three aliquots (replicates) each of nine samples were digested in a single microwave run, along with a replicate SRM NIST 1570a, heroin control and a method blank. Approximately 30-100 mg of each sample or control were weighed directly into individual quartz micro-insert vials to the nearest $0.1 \mathrm{mg}$ on a Mettler AE240 (Mettler Toledo, USA) balance using disposable polypropylene (PPE) spatulas to transfer the samples from the original sample containers. The quartz micro-insert vials were immediately topped with polyether ketone (PEK) caps, which were only removed briefly for the addition of internal standards and nitric acid to minimize exposure to the open-air environment. All of the sample manipulation was conducted inside class-100 fume hoods wearing appropriate personal protective equipment as well as covering the hair and mouth to protect the sample against contamination.

To each quartz vial, including one empty vial to be used as the method blank, a digestion internal standard was spiked by electronic pipette (Ovation ${ }^{\circledR}$ Macro10; Vistalabs, USA). This standard consisted of $200 \mu \mathrm{L}$ of $200 \mathrm{ppb}{ }^{6} \mathrm{Li}, \mathrm{Sc}, \mathrm{Y}, \mathrm{In}, \mathrm{Tb}$ and $\mathrm{Bi}$ in $0.8 \mathrm{M}$ $\mathrm{HNO}_{3}$ (diluted from 71D). This spike was added both to be used as digestion internal 
standards as well as to pre-wet the sample before adding acids. Occasionally the dry powder samples would stick to the inside of the micro-insert vessels and such cases, the internal standard aliquot was directed to rinse the sample down to the bottom of the vial. In the event of any sample losses during solution transfer and recover, these standards could be used adjust the calculation of mass dilution. Lastly, $2.3 \mathrm{ml}$ of Optima grade nitric acid was added to all samples, rinsing any remaining solids down from the walls and ensuring that the entire sample was wet and in contact with the acid solution.

For each microwave digestion run, ten polyether ether ketone (PEEK) digestion vessels (SK-15; Milestone, Scientific, USA) were used, each containing a buffer solution of $10 \mathrm{~mL} 20 \% \mathrm{v} / \mathrm{v} \mathrm{H}_{2} \mathrm{O}_{2}$ (ACS grade, Fisher Chemical) and three quartz vials carefully loaded with clean plastic tweezers. Vessels 2-10 contained heroin samples (three replicates per vessel). Vessel 1 (the reference vessel) contained one replicate each of spinach control NIST SRM 1570a, heroin control GFX2-83/8200-9, and the method blank.

The buffer solution never came in contact with the sample solutions and was present to regulate the temperature within each vessel as well as to assist in the conversion of $\mathrm{NO}_{\mathrm{x}}$ (g) back in to $\mathrm{HNO}_{3}$. Without $\mathrm{H}_{2} \mathrm{O}_{2}$ present in the outer buffer solution, the vessels released a substantial about of noxious, yellow $\mathrm{NO}_{\mathrm{x}(\mathrm{g})}$ fumes to be vented post-digestion. The method described resulted in no colored vapors and digested solutions with total visual clarity. The chemical reaction for the oxidation of covalent carbon bonds by nitric acid is shown in Equation 1 along with three reactions governing the convergence of nitric and nitrous oxides ultimately back into nitric acid via reaction with hydrogen peroxide are shown in the following equations[47]. 
Equation 1: chemical reactions of carbon bond oxidation by $\mathrm{HNO}_{3}$ and remediation of $\mathrm{NO}$ by way of converting $\mathrm{HNO}_{2}$ to $\mathrm{HNO}_{3}$ by $\mathrm{H}_{2} \mathrm{O}_{2}$ [47].

$$
\begin{gathered}
\left(\mathrm{CH}_{2}\right)_{\mathrm{x}}+2 \mathrm{x}\left(\mathrm{HNO}_{3}\right) \rightarrow \mathrm{xCO}+2 \mathrm{xNO}+2 \mathrm{xH}_{2} \mathrm{O} \\
3 \mathrm{NO}_{2}+\mathrm{H}_{2} \mathrm{O} \Leftrightarrow 2 \mathrm{HNO}_{3}+\mathrm{NO} \\
2 \mathrm{NO}+\mathrm{HNO}_{3}+\mathrm{H}_{2} \mathrm{O} \rightarrow 3 \mathrm{HNO}_{2} \\
\mathrm{HNO}_{2}+\mathrm{H}_{2} \mathrm{O}_{2} \rightarrow \mathrm{HNO}_{3}+\mathrm{H}_{2} \mathrm{O}
\end{gathered}
$$

After loading, the vessels were assembled and sealed to a specification of $10 \mathrm{~N} \mathrm{~m}$ with a torque wrench supplied by Milestone and then loaded into position in the microwave oven with the thermal probe positioned into the reference vessel. EPA method 3052 requires that a thermal probe be used in at least one vessel [45]. The microwave continually adjusts its power depending upon the temperature in the reference vessel to within $\pm 1 \mathrm{~W}$ in less than one second. The maximum power of the microwave was optimized for the number of samples used. If the power is too high the pressure will build up too rapidly, leading to venting and possible sample loss. If the power is too low, the internal temperature of the vessels will not reach the prescribed temperature within the ramp time, resulting in incomplete digestion. For 10 vessels (30 individual samples), a maximum power of 1600 Watts was used.

The optimized method (see Table 3) was a 15-minute ramp from room temperate to $220{ }^{\circ} \mathrm{C}$ followed by a hold at $220{ }^{\circ} \mathrm{C}$ for an additional 15 minutes. Following the digestion program, the microwave's ventilation fan allowed the samples to cool slowly to $\leq 40{ }^{\circ} \mathrm{C}$ to avoid rapid depressurization of the vessel, which could lead to loss of sample. 
Table 3: Microwave parameters for a batch of 30 heroin samples

1. Ramp to $220^{\circ} \mathrm{C}$ in $15 \mathrm{~min}, 1600$ Watts of max power

2. Hold at $220^{\circ} \mathrm{C}$ for $15 \mathrm{~min}, 1600$ Watts of max power

3. Cool for $90 \mathrm{~min}$ or until internal temperature is $\leq 40{ }^{\circ} \mathrm{C}$

Following digestion, the vessels were removed from the microwave oven once the internal temperature had reached $\leq 40^{\circ} \mathrm{C}$ (60-90 minutes was typically sufficient). Using the torque wrench, the seals were slowly released and the PEK inserts that held the quartz insert vials were carefully removed with plastic tweezers.

Pre-weighed, $15 \mathrm{~mL}$ polypropylene centrifuge tubes (Corning, USA) were used to collect the digestion product from the quartz vials. A centrifuge tube was set around the opening of the quartz vial and then both were inverted to transfer the sample to the centrifuge tube without loss. Boron-free DI $\mathrm{H}_{2} \mathrm{O}$ from a clean wash bottle was used to rinse the vial approximately 3 times into the centrifuge tube, which yielded approximately $13 \mathrm{~mL}$ of diluted solution. The final mass was determined by weighing by difference of the centrifuge tubes to the nearest $0.01 \mathrm{~g}$ on a Sartorius LC4200 balance (Sartorius AG, Germany). The masses were converted to volumes using the average density, which was determined to be $1.082 \mathrm{~g} / \mathrm{mL}$ (see subchapter 2.3.7).

Trace metal grade $(\mathrm{TMG})$ acids (Fisher Scientific, USA) and > $18 \mathrm{M} \Omega$ Evoqua ${ }^{\circledR}$ DI $\mathrm{H}_{2} \mathrm{O}$ (Evoqua, USA) were used to prepare all acid leaching baths. Reused lab-ware, such as PEK caps for the quartz vials, Teflon ${ }^{\circledR}$ beakers, plastic tweezers, and plastic spatulas were thoroughly rinsed with DI $\mathrm{H}_{2} \mathrm{O}$, then leached in $1: 1 \mathrm{v} / \mathrm{v}$ DI: $\mathrm{HNO}_{3}(\sim 6 \mathrm{M}$ 
$\mathrm{HNO}_{3}$ ) overnight. The following day they were transferred to a $5 \% \mathrm{HCl}$ bath and left overnight. After a final rinse in $\mathrm{DI} \mathrm{H}_{2} \mathrm{O}$, they were allowed to dry on a clean plastic rack in a Class 100 clean lab before use.

Following a digestion, the quartz micro-insert vials were first cleaned by a thorough DI $\mathrm{H}_{2} \mathrm{O}$ rinse and mild agitation with a cotton-tipped applicator and a final rinse in $\mathrm{DI} \mathrm{H}_{2} \mathrm{O}$. If the quartz vials required heavy cleaning, the vials were bathed overnight in aqua regia, prepared by carefully adding $370 \mathrm{~mL} \mathrm{HCl}$ to $100 \mathrm{~mL} \mathrm{HNO}_{3}$, both of trace metal grade. Usually the vials appeared very clean following digestions, and were routinely cleaned by soaking overnight in $1: 1 \mathrm{HNO}_{3}$ and $\mathrm{DI}_{2} \mathrm{O}$ at $90^{\circ} \mathrm{C}$. The next day the vials were removed, rinsed with $\mathrm{DI} \mathrm{H}_{2} \mathrm{O}$, and allowed to dry on a cleaned plastic rack in a Class 100 clean lab before reuse. Aqua regia is aggressively corrosive and produces noxious fumes when prepared. It should not be stored for longer than one week because it becomes unstable. It requires roughly $4 \mathrm{~L}$ of a saturated $\mathrm{NaHCO}_{3}$ solution to neutralize $500 \mathrm{~mL}$ of aqua regia, which should be done with copious amounts of ice inside of a fume hood for safety.

\subsubsection{Q-ICP-MS Method}

The most basic configuration of a modern ICP-MS instrument is the quadrupole mass analyzer. The instrument used for the preliminary elemental analysis in this research was the Agilent 7700X quadrupole ICP-MS (see Figure 5). In addition to the instrument specifications described in subchapter 2.2.2, it is equipped with an octapole reaction/collision cell that can be operated in normal (NO GAS), Hydrogen (H2) and Helium (He) modes depending upon the anticipated isobaric interferences for a particular 
analyte. A full list of analyte elements and the corresponding mode of operation can be found in Table 4.

Typically, He mode was used for transition metals as their primary matrix interferences were expected to be polyatomic ions such as $\mathrm{X}+\mathrm{Cl}^{+}$and $\mathrm{Ar}_{2}{ }^{+}$. Helium has a wider atomic radius and interacts with molecules in the octapole via collision. Collisioninduced dissociation is the accepted mechanism by which helium interacts to suppress the signal of isobaric interferences. Elements such as arsenic $\left({ }^{75} \mathrm{As}\right)$ were measured in He mode because chloride complexes were the most problematic interferences (e.g., $\left.{ }^{75} \mathrm{ArCl}\right)$.

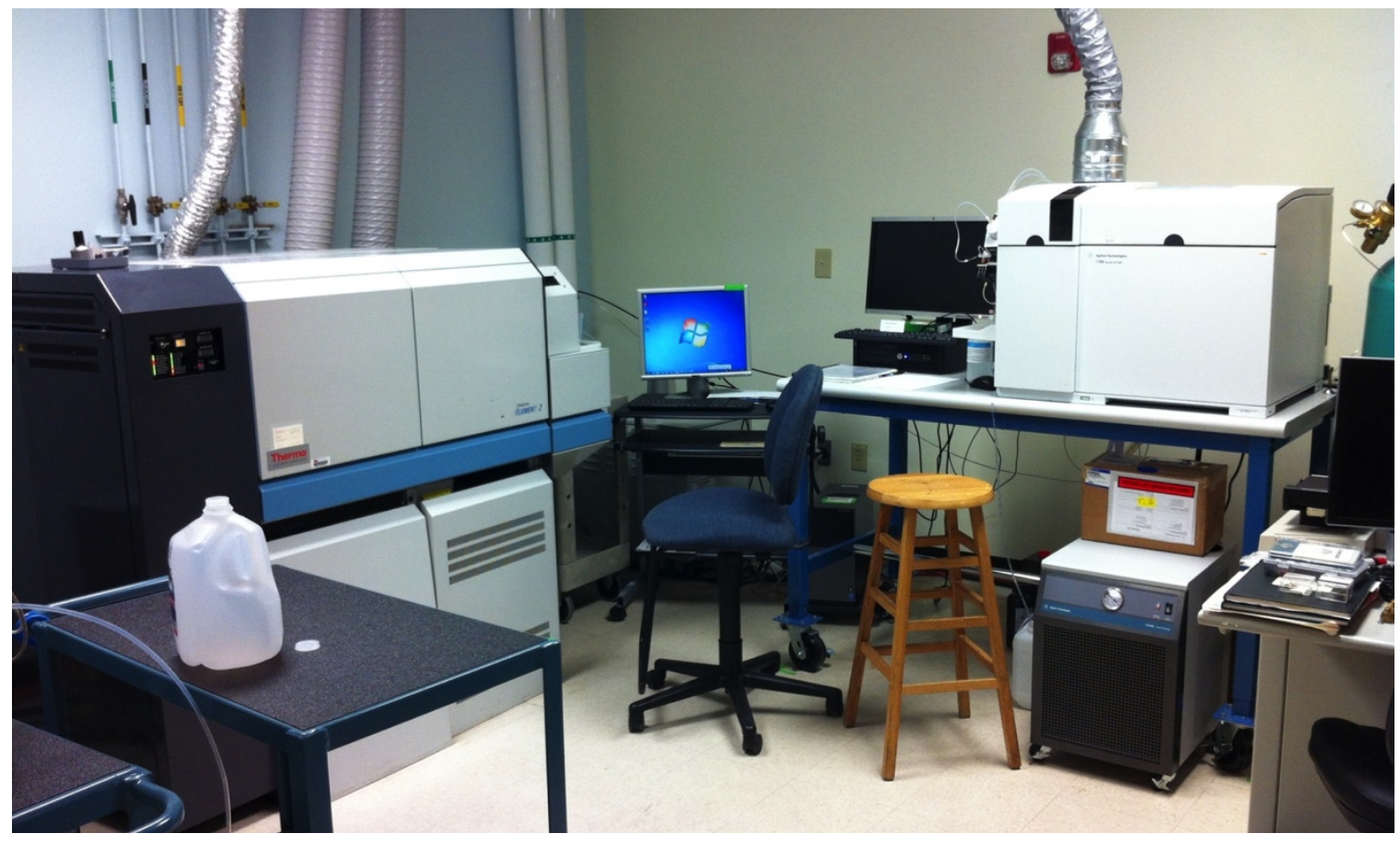

Figure 5. Thermo Element 2 HR-ICP-MS (left) and Agilent 7700x Q-ICP-MS (right) in the Trace Evidence Analysis Facility (TEAF) at FIU.

Hydrogen (H2) mode was employed to reduce the signal for plasma-based interferences. Oxides of lighter elements $\mathrm{X}+\mathrm{O}^{+}$, often pose an issue for the measurement 
of heavier analytes where $\mathrm{X}$ is an isotope $16 \mathrm{u}$ lighter than the isotope of interest. Hydrogen is a reaction gas and the accepted mechanism by which $\mathrm{H}_{2}$ interacts with isobaric interferences is through charge-transfer neutralization of the interfering species. Neutral species cannot be analyzed in the mass analyzer region and hence no longer interfere with the measurement. Rare earth elements were monitored in $\mathrm{H} 2$ mode as $\mathrm{X}+\mathrm{O}^{+}$interferences were expected.

\begin{tabular}{|c|c|c|c|c|c|c|c|c|}
\hline \multicolumn{3}{|c|}{ No Gas Mode } & \multicolumn{3}{|c|}{ Hydrogen (H2) Mode } & \multicolumn{3}{|c|}{ Helium (He) Mode } \\
\hline \multicolumn{2}{|c|}{ Isotope } & \multirow{2}{*}{$\begin{array}{l}\begin{array}{l}\text { Integration } \\
\text { (s) }\end{array} \\
0.09\end{array}$} & \multicolumn{2}{|c|}{ Isotope } & \multirow{2}{*}{$\begin{array}{l}\text { Integration } \\
\text { (s) } \\
0.3\end{array}$} & \multicolumn{2}{|c|}{ Isotope } & \multirow{2}{*}{$\begin{array}{l}\text { Integration } \\
\text { (s) }\end{array}$} \\
\hline & $\mathrm{Li}$ & & & $\mathrm{K}$ & & & $\mathrm{Mg}$ & \\
\hline 7 & $\mathrm{Li}$ & 0.09 & & $\mathrm{~V}$ & 0.3 & 27 & $\mathrm{Al}$ & 0.3 \\
\hline 9 & $\mathrm{Be}$ & 0.09 & 72 & $\mathrm{Ge}$ & 0.3 & 45 & $\mathrm{Sc}$ & 0.3 \\
\hline 10 & $\mathrm{~B}$ & 0.09 & 78 & $\mathrm{Se}$ & 0.51 & 47 & $\mathrm{Ti}$ & 0.3 \\
\hline 11 & B & 0.09 & & $\mathrm{Rh}$ & 0.3 & 52 & $\mathrm{Cr}$ & 0.3 \\
\hline 23 & $\mathrm{Na}$ & 0.09 & & $\mathrm{Nd}$ & 0.3 & 57 & $\mathrm{Fe}$ & 0.3 \\
\hline 31 & $\mathrm{P}$ & 0.09 & & $\mathrm{Sm}$ & 0.3 & 59 & Co & 0.3 \\
\hline 43 & $\mathrm{Ca}$ & 0.09 & & $\mathrm{Eu}$ & 0.3 & 60 & $\mathrm{Ni}$ & 0.3 \\
\hline 55 & $\mathrm{Mn}$ & 0.09 & & $\mathrm{Gd}$ & 0.3 & 63 & $\mathrm{Cu}$ & 0.3 \\
\hline 85 & $\mathrm{Rb}$ & 0.09 & & Dy & 0.3 & 66 & $\mathrm{Zn}$ & 0.3 \\
\hline 88 & $\mathrm{Sr}$ & 0.09 & 165 & Ho & 0.3 & 71 & $\mathrm{Ga}$ & 0.3 \\
\hline 89 & $\mathrm{Y}$ & 0.09 & 166 & $\mathrm{Er}$ & 0.3 & 75 & As & 0.51 \\
\hline 90 & $\mathrm{Zr}$ & 0.09 & 169 & $\mathrm{Tm}$ & 0.3 & 95 & Mo & 0.3 \\
\hline 93 & $\mathrm{Nb}$ & 0.09 & 172 & $\mathrm{Yb}$ & 0.3 & 103 & $\mathrm{Rh}$ & 0.3 \\
\hline 103 & $\mathrm{Rh}$ & 0.3 & 175 & $\mathrm{Lu}$ & 0.3 & 107 & $\mathrm{Ag}$ & 0.3 \\
\hline 115 & In & 0.09 & & $\mathrm{Hf}$ & 0.3 & 111 & $\mathrm{Cd}$ & 0.3 \\
\hline 118 & $\mathrm{Sn}$ & 0.09 & & $\mathrm{Ta}$ & 0.3 & 133 & Cs & 0.3 \\
\hline 121 & $\mathrm{Sb}$ & 0.09 & & W & 0.3 & & & \\
\hline 125 & $\mathrm{Te}$ & 0.09 & 197 & $\mathrm{Au}$ & 0.3 & & & \\
\hline 137 & $\mathrm{Ba}$ & 0.09 & & & & & & \\
\hline 139 & $\mathrm{La}$ & 0.09 & & & & & & \\
\hline 140 & $\mathrm{Ce}$ & 0.09 & & & & & & \\
\hline 141 & $\operatorname{Pr}$ & 0.09 & & & & & & \\
\hline 159 & $\mathrm{~Tb}$ & 0.09 & & & & & & \\
\hline 205 & $\mathrm{Tl}$ & 0.09 & & & & & & \\
\hline 208 & $\mathrm{~Pb}$ & 0.09 & & & & & & \\
\hline 209 & $\mathrm{Bi}$ & 0.09 & & & & & & \\
\hline 232 & Th & 0.09 & & & & & & \\
\hline 238 & $\mathrm{U}$ & 0.09 & & & & & & \\
\hline
\end{tabular}


Calibration solutions were prepared from ICP-MS multielement mixtures (Inorganic Ventures, USA) at $0.03,0.1,0.3,1.0,3.0,10,30$ and $100 \mu \mathrm{g} / \mathrm{L}$ in $0.8 \mathrm{M} \mathrm{HNO}_{3}$. To ensure the accuracy and stability of the calibration, QC standards from a third party vendor (High-purity Standards, USA) were used. They were prepared at $2.0(\mathrm{QC1})$ and 20 (QC2) $\mu \mathrm{g} / \mathrm{L}$ in $0.8 \mathrm{M} \mathrm{HNO}_{3}$. To account for the instrumental variation associated with sample introduction and plasma inconsistencies, internal standards were used. A $10 \mu \mathrm{g} / \mathrm{L}$ $\mathrm{Rh}$ solution was introduced inline via an integrated mixing tee and measured as ${ }^{103} \mathrm{Rh}$. A reagent blank of $0.8 \mathrm{M} \mathrm{HNO}_{3}$ was also prepared.

\begin{tabular}{|c|c|c|c|}
\hline Parameter & Value & Parameter & Value \\
\hline Peristaltic pump & $0.1 \mathrm{rps}$ & Extraction lens 1 & $0 \mathrm{~V}$ \\
\hline Sample gas & $1.00 \mathrm{~L} / \mathrm{min}$ & Extraction lens 2 & $-190 \mathrm{~V}$ \\
\hline Dilution gas & $0.13 \mathrm{~L} / \mathrm{min}$ & Omega Bias & $-70 \mathrm{~V}$ \\
\hline Spray chamber $\mathrm{T}$ & $2^{\circ} \mathrm{C}$ & Omega Lens & $7.6 \mathrm{~V}$ \\
\hline RF power & $1550 \mathrm{~W}$ & He flow rate & $5.0-5.2 \mathrm{~mL} / \mathrm{min}$ \\
\hline Detector mode & Both & $\mathrm{H}_{2}$ flow rate & $4.6 \mathrm{~mL} / \mathrm{min}$ \\
\hline
\end{tabular}

Instrumental parameters are listed on Table 5. Note that tuning was done daily to optimize the instrumental parameters for high signal, low oxides, low doubly-charged levels, and low RDSs so the actual values on a given day may vary slightly.

The sample sequence was set up in the following order: reagent blank, calibration standards (in order of increasing concentration), reagent blank, QC1, QC2, reagent blank, digestion internal standard solutions, reagent blank, diluted digest solutions (method blank, heroin control, spinach control, samples), reagent blank QC1, QC2, and calibrations standards once again as samples. Before measuring a sample, the sample introduction system was flushed with the rinse solution or $0.1 \%$ Triton-X (MilliporeSigma, USA) in 
$0.8 \mathrm{M} \mathrm{HNO}_{3}$ to prevent carry-over from the previous sample, followed by a 20 second takeup and 15 second stabilization time to bring a stable, homogeneous sample aerosol into the plasma.

The analysis software performs the following operations to convert the measured intensities to element concentrations in solution: normalization of all samples to the internal standard, subtraction of the reagent blank signal, linear regression of the calibration standards to build a calibration curve, and calculation of the concentrations of each sample solution from the linear regression equations from the calibration samples. Each sample was measured in three replicates, which were used by the software to calculate the mean, standard deviation, and relative standard deviation of each sample. The instrument's software calculates the limits of detection (LOD) for each element. The method LOD for each sample was determined by multiplying the sample's dilution factor by the average instrumental LOD for each element; the method LOD was lowest value reported for any element of a sample.

\subsubsection{HR-ICP-MS Method}

High resolution ICP-MS analysis was performed using the Element 2 (Thermo Electron, Germany) shown on Figure 5, is a sector field ICP-MS. The instrument is more sensitive and has much better resolution than quadrupole instruments. However, it is also more costly to purchase and more expensive to maintain than quadrupole instruments. It also requires a greater degree of expertise to correctly operate a high-resolution ICP-MS instrument and to achieve accurate, precise and reproducible results as compared to a quadrupole ICP-MS. 
The main strategy the Element 2 uses to deal with interferences is to increase the mass resolution. The mass resolution of a peak is the mass of the peak center divided by the peak width at $5 \%$ of its height. As the peaks are narrowed and the mass separation is improved, the mass resolution increases and improved separation between neighboring peaks is achieved. There are two ways by which this is achieved: using slits and a doublefocusing magnetic-electrostatic sector mass separator in reversed Nier-Johnson geometry (see Figure 6).

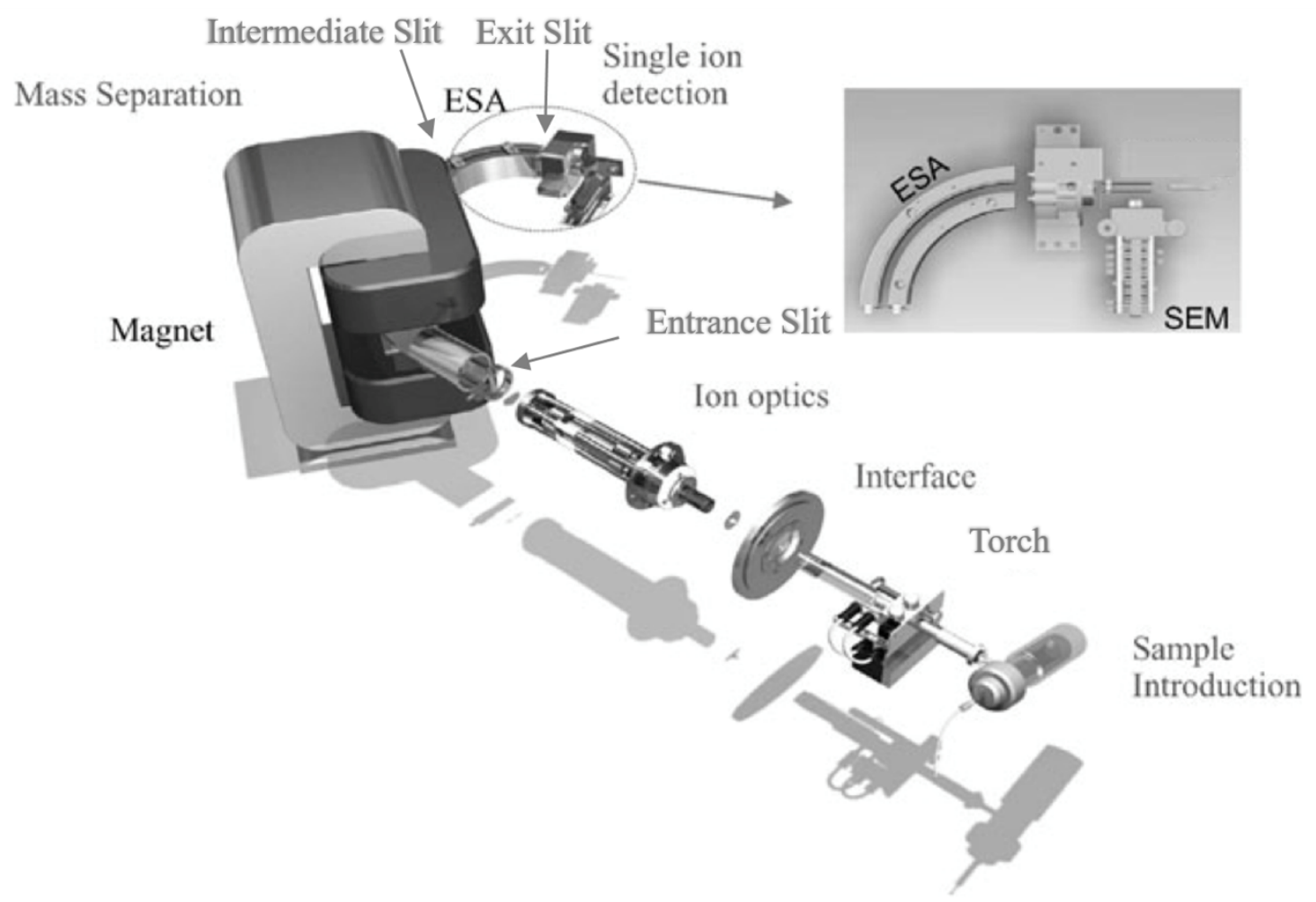

Figure 6. Schematic of a Thermo Element 2 HR-ICP-MS with reversed Nier-Johnson geometry. Modified from [48].

The Element 2 has three slits: an entrance slit between the ion optics and the magnetic sector, an intermediate slit between the magnetic and electrostatic sectors, and an exit slit between the electrostatic analyzer (ESA) and the detector. The entrance slit is used 
to focus and narrow the beam more than is possible by the ion optics alone. Ions exiting from the entrance slit enter into the magnetic field produced in the curved magnetic sector, which imposes a force on the ions perpendicular to the direction of motion and thus a curved flight path. The ions will get dispersed by their momentum (energy and mass) until they reach an intermediate slit. At a given velocity and magnetic field induction, only ions with a specific $\mathrm{m} / \mathrm{z}$ will have the correct curved trajectory to make it through the intermediate slit into the ESA. In the ESA, a direct current is applied to the ESA inner and outer plates giving the inner plate a negative polarity attracting the positive ions, while the outer plate (positive polarity) repels them. The ions directed towards the ESA get dispersed with respect to their energy only and are then refocused onto the exit slit leading into the detector.
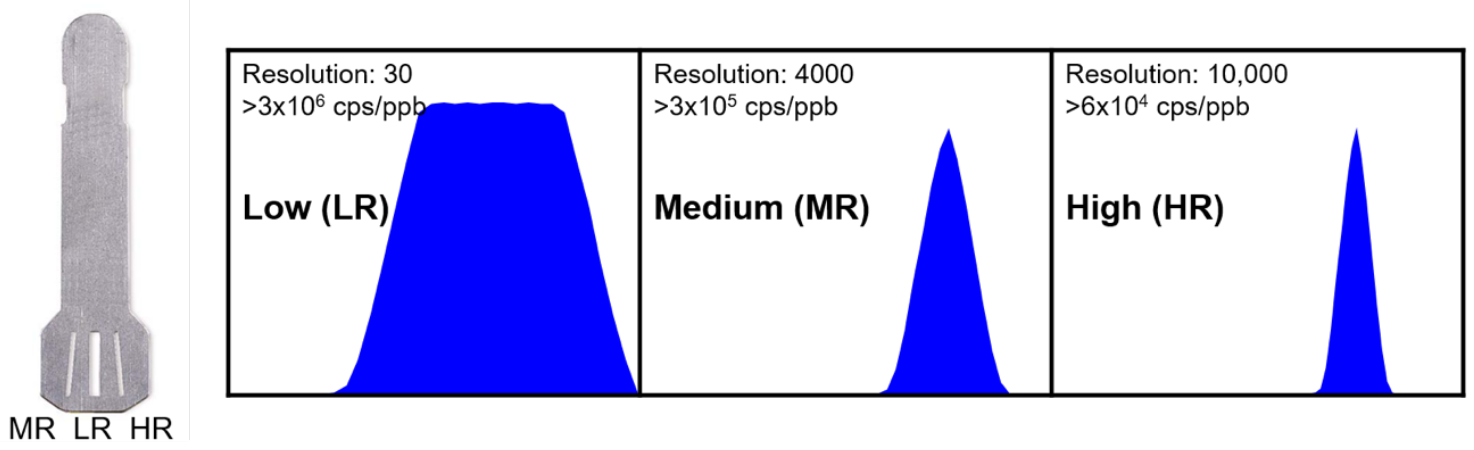

Figure 7. Left: moveable slits in the Thermo Element 2 HR-ICP-MS. Right: comparison of the peak shape, resolution, and sensitivity of the three mass resolutions, modified from [48].

The width of the entrance and exit slits can be set for low, medium, or high (the narrowest width) resolution (LR, MR, and HR, respectively, see Figure 7). Low resolution is capable of a resolution of approximately 300 (in contrast with a quadrupole which has a resolution of approximately 1), MR 4000, and HR 10,000. Note that the narrower the slit, 
the fewer ions pass through it, so there is a loss in sensitivity. However, the sensitivity of the Element 2 in LR is still 1-2 orders of magnitude greater than that of a quadrupole (which is comparable to the sensitivity of the Element 2 in MR).

The nominal masses described earlier are simply rounded to the nearest whole number, but the exact masses may be different enough to be resolved by a high-resolution instrument such as the Element 2. For example, the exact mass of ${ }^{75} \mathrm{As}$ is $74.921597 \mathrm{u}$, while the exact mass of the ${ }^{40} \mathrm{Ar}^{35} \mathrm{Cl}$ is $74.931235 \mathrm{u}$, which can be separated with a resolution of 7774 or greater, is easily achieved in HR, shown in Figure 8.

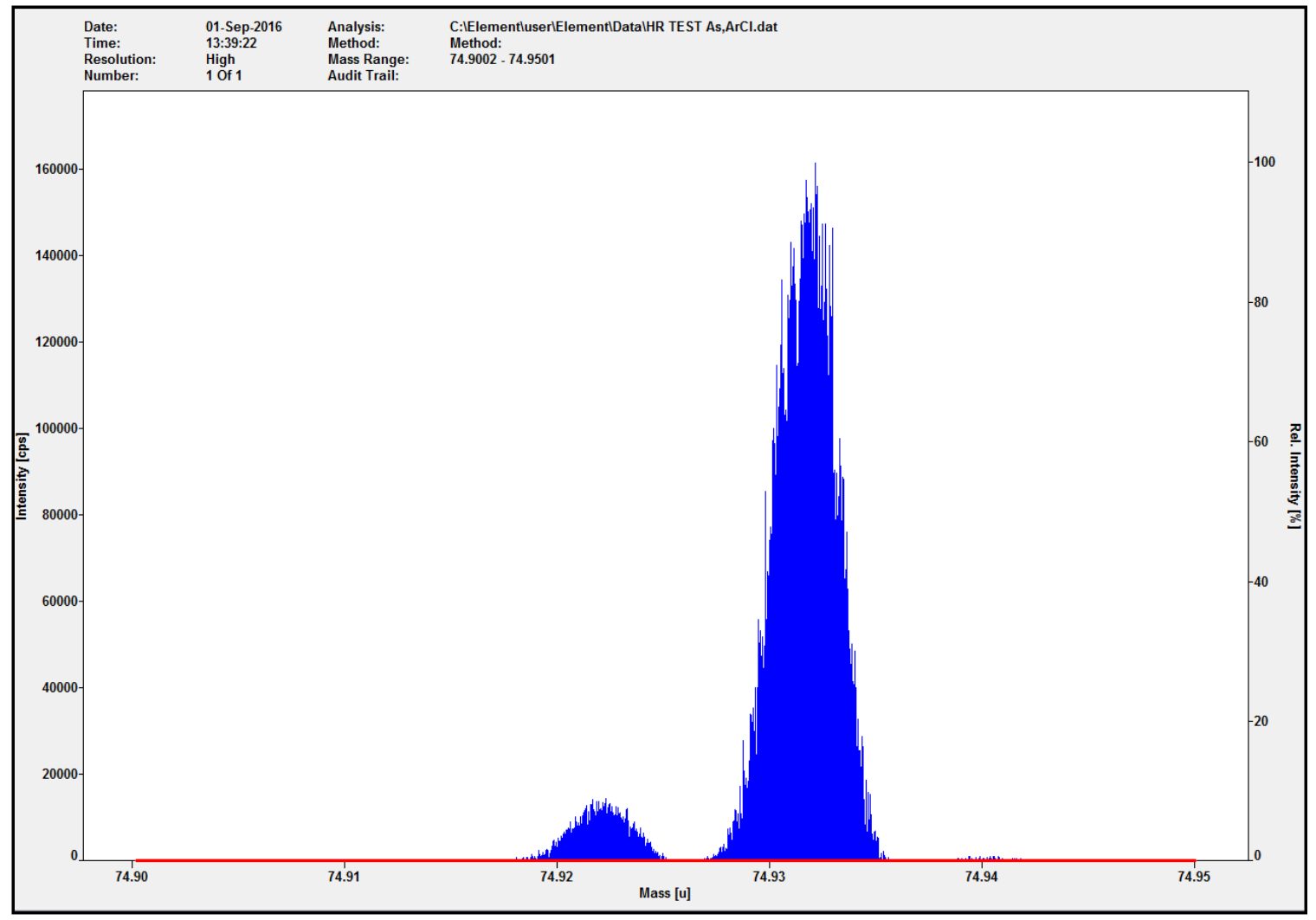

Figure 8. Baseline resolution of ${ }^{75} \mathrm{As}^{+}$(left) from the $\left({ }^{40} \mathrm{Ar}{ }^{35} \mathrm{Cl}\right)^{+}$interference (right) in high resolution by the Thermo Fisher Element $2 \mathrm{HR}-\mathrm{ICP}-\mathrm{MS}$ in a solution of $4 \mathrm{ppb} \mathrm{As}, 0.08 \mathrm{M} \mathrm{HCl}$ and $0.6 \mathrm{M} \mathrm{HNO}$. 
Calibration solutions were prepared at $0,0.003,0.03,0.3,3$, and, $30 \mu \mathrm{g} / \mathrm{L}$ in $0.8 \mathrm{M}$ $\mathrm{HNO}_{3}$. To ensure the accuracy and stability of the calibration, quality control (QC1 and QC2) standard mixtures were used. They were prepared at 2.0 (QC1) and $20(\mathrm{QC} 2) \mu \mathrm{g} / \mathrm{L}$ in $0.8 \mathrm{M} \mathrm{HNO}_{3}$. To account for the instrumental variation associated with sample introduction and plasma inconsistencies, internal standards were used. A $10 \mu \mathrm{g} / \mathrm{L} \mathrm{Rh}$ solution was introduced inline via a mixing tee (Glass Expansion, USA) and measured as ${ }^{103} \mathrm{Rh}$. A reagent blank was prepared from the $0.8 \mathrm{M} \mathrm{HNO}_{3}$ used for preparing the above solutions.

The following isotopes were measured in low resolution: ${ }^{9} \mathrm{Be},{ }^{11} \mathrm{~B},{ }^{23} \mathrm{Na},{ }^{53} \mathrm{Cr},{ }^{85} \mathrm{Rb}$, ${ }^{88} \mathrm{Sr},{ }^{90} \mathrm{Zr},{ }^{93} \mathrm{Nb},{ }^{95} \mathrm{Mo},{ }^{107} \mathrm{Ag},{ }^{111} \mathrm{Cd},{ }^{118} \mathrm{Sn},{ }^{121} \mathrm{Sb},{ }^{125} \mathrm{Te},{ }^{133} \mathrm{Cs},{ }^{137} \mathrm{Ba},{ }^{139} \mathrm{La},{ }^{140} \mathrm{Ce},{ }^{141} \mathrm{Pr}$, ${ }^{146} \mathrm{Nd},{ }^{147} \mathrm{Sm},{ }^{153} \mathrm{Eu},{ }^{157} \mathrm{Gd},{ }^{163} \mathrm{Dy},{ }^{165} \mathrm{Ho},{ }^{166} \mathrm{Er},{ }^{169} \mathrm{Tm},{ }^{172} \mathrm{Yb},{ }^{175} \mathrm{Lu},{ }^{178} \mathrm{Hf},{ }^{182} \mathrm{~W},{ }^{205} \mathrm{Tl},{ }^{208} \mathrm{~Pb}$, ${ }^{232} \mathrm{Th},{ }^{238} \mathrm{U}$. The following isotopes were measured in medium resolution: ${ }^{24} \mathrm{Mg},{ }^{27} \mathrm{Al},{ }^{31} \mathrm{P}$, ${ }^{32} \mathrm{~S},{ }^{43} \mathrm{Ca},{ }^{44} \mathrm{Ca},{ }^{47} \mathrm{Ti},{ }^{51} \mathrm{~V},{ }^{52} \mathrm{Cr},{ }^{53} \mathrm{Cr},{ }^{55} \mathrm{Mn},{ }^{56} \mathrm{Fe},{ }^{59} \mathrm{Co},{ }^{60} \mathrm{Ni},{ }^{63} \mathrm{Cu},{ }^{66} \mathrm{Zn},{ }^{69} \mathrm{Ga}$. The following isotopes were measured in high resolution: ${ }^{39} \mathrm{~K},{ }^{72} \mathrm{Ge},{ }^{75} \mathrm{As},{ }^{77} \mathrm{Se}$. Each of the elements measured by HR-ICP-MS were also measured by Q-ICP-MS to cross-check the performance between the two instruments.

Instrumental method parameters for the Thermo Fisher Element 2 HR-ICP-MS are listed on Table 6. Note that tuning was done daily to optimize the instrumental parameters for achieving high signal intensity, low oxides formation, low doubly-charged species, and signal stability so the actual values on a given day may vary slightly. The sequence and data processing were very similar in principle to that done for the Q-ICP-MS analysis (see subchapter 2.3.4. 
Table 6. Instrumental parameters for HR-ICP-MS measurements using the Thermo Element 2.

\begin{tabular}{|c|c|c|c|}
\hline Parameter & Value & Parameter & Value \\
\hline Peristaltic pump & $6 \mathrm{rpm}$ & Extraction lens & $-2000 \mathrm{~V}$ \\
\hline Sample gas & $0.98 \mathrm{~L} / \mathrm{min}$ & Focus lens & $-900 \mathrm{~V}$ \\
\hline Auxiliary gas & $0.9 \mathrm{~L} / \mathrm{min}$ & Shape lens & $120 \mathrm{~V}$ \\
\hline Cool gas & $16 \mathrm{~L} / \mathrm{min}$ & MS mode & Mass Accuracy \\
\hline $\mathrm{Z}$ position & $-3 \mathrm{~mm}$ & Scan mode & E-Scan \\
\hline RF power & $1200 \mathrm{~W}$ & Detector mode & Both \\
\hline
\end{tabular}

\subsubsection{Reagents and Elemental Standards}

For all sample preparation and digestion, Optima grade nitric acid (Fisher Scientific, USA) was used. All deionized (DI) water used for preparation of samples, standards, and blanks was obtained at $>18 \mathrm{M} \Omega$ from a Millipore Milli-Q systems (EMD Millipore, Germany) equipped with a boron-free filter to achieve low boron levels, as well as the lowest possible levels of other trace metals.

Calibration standards for ICP-MS were prepared from stock solutions of ICP-MS Mix 71A (Ag, Al, As, B, Ba, Be, Ca, Cd, Ce, Co, Cr(III), Cs, Cu, Dy, Er, Eu, Fe, Ga, Gd, Ho, K, La, Lu, Mg, Mn, Na, Nd, Ni, P, Pb, Pr, Rb, S, Se, Sm, Sr, Th, Tl, Tm, U, V, Yb, and $\mathrm{Zn}$ ), 71B (Ge, Hf, Mo, Nb, Sb, Si, Sn, Ta, Te, Ti, W, and Zr), 71D (Bi, In, ${ }^{6} \mathrm{Li}, \mathrm{Sc}, \mathrm{Tb}$, and $\mathrm{Y}$ ) and $\mathrm{Au}$ single element solution (Inorganic Ventures, USA). Internal digestion standard solutions were prepared from stock solutions of ICP-MS Mix 71D. Instrumental calibration standard was a $100 \mu \mathrm{g} / \mathrm{L}$ dilution in $0.8 \mathrm{M} \mathrm{HNO}_{3}$ of rhodium single element standard (Inorganic Ventures, USA). Solutions of Al, B, Ba, Ca, Dy, K, Mo, Pb, Se, Sn, $\mathrm{Sr}, \mathrm{Ti}, \mathrm{Tl}, \mathrm{Zn}$ used for quality control standards (QC) solutions were purchased from a secondary source (High Purity Standards, USA). ICP-MS rinse solutions were prepared 
with $0.1 \%$ Triton-X (Fisher Scientific, USA) in $2 \%$ trace metal grade nitric acid (Fisher Scientific, USA).

\subsubsection{Characterization of Sample Post-Digestion}

The samples were characterized for both the acid molarity to calculate the appropriate dilution factor to prepare the samples for analysis by ICP-MS. Even though the instrument may tolerate high molar acids, it does accelerate the pace of maintenance. In addition, the acid matrix of calibration solutions should be matched as closely as possible to that of the samples being analyzed. It would consume expensive acid reagents to prepared them in higher concentrations, so it was determined that dilution of the sample was more cost effective. In addition, dilution of the samples would afford an opportunity to partition the samples and reduce contamination to the main portion of the sample given the need to reanalyze the sample. This made it also suitable for an aliquot of the sample to be reserved for analysis for ${ }^{87} \mathrm{Sr} /{ }^{86} \mathrm{Sr}$ isotope analysis as well, which is described in Chapter 4.

The results of the titration are shown on Figure 9, wherein samples over a range of sample masses were analyzed for their resulting nitric acid concentration. The titration results suggest that using lesser sample masses yields a higher final acid molarity postdigestion. Not surprisingly, the titration also demonstrated that using a larger volume of concentrated nitric acid allows for a larger mass of heroin sample to be digested, while still resulting in equitable acid molarities in the post-digestion solution. The target mass range was set at approximately 50 - $80 \mathrm{mg}$ therefore the final acid molarity was assumed to be 
1.4 - $1.8 \mathrm{M} \mathrm{HNO}_{3}$ and the final dilution ratio was $1: 1$, where $3 \mathrm{~mL}$ diluted to a final volume of $6 \mathrm{~mL}$.

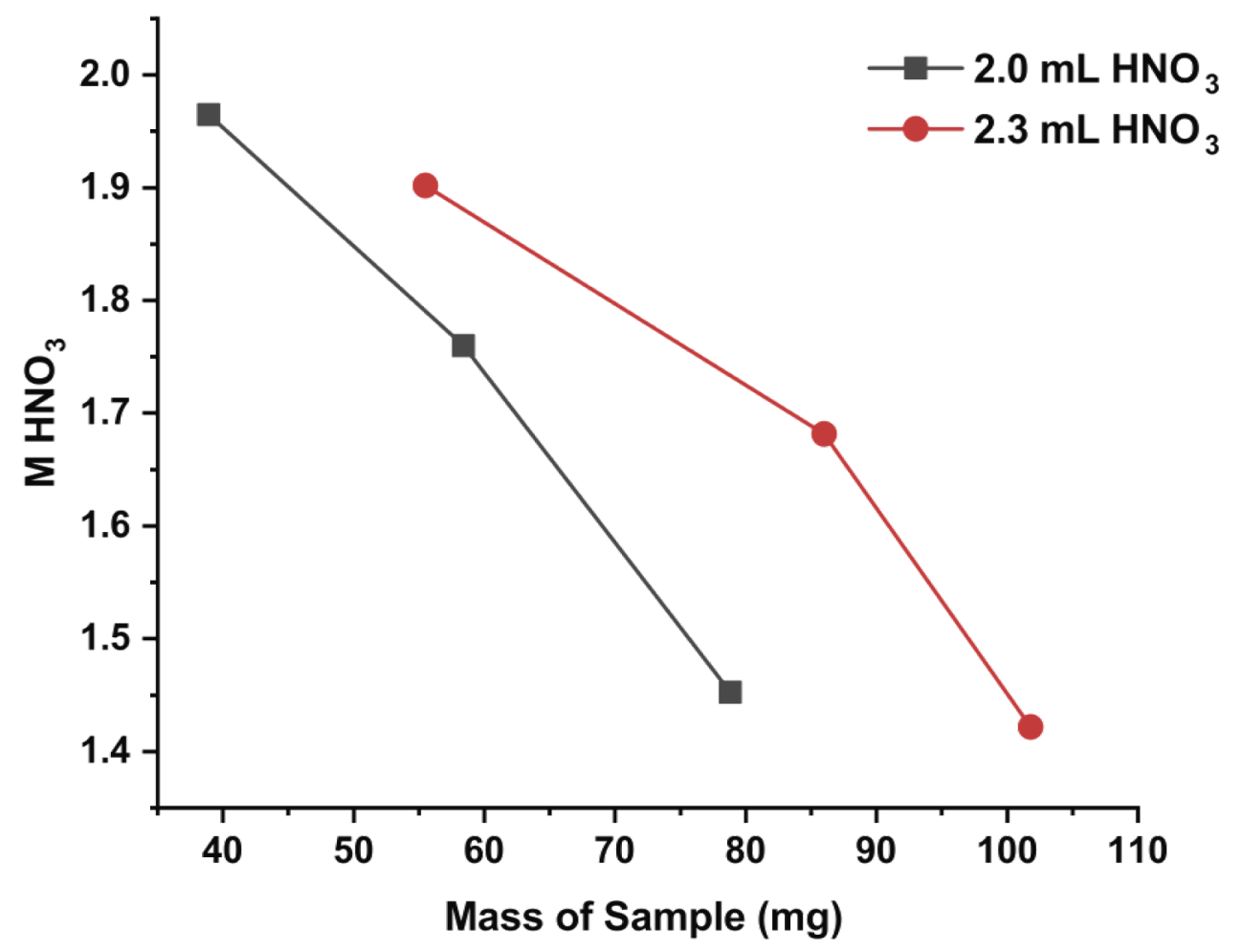

Figure 9. Titration with $\sim 0.1 \mathrm{M} \mathrm{NaOH}$ solution of heroin samples to determine sample mass/post-digest acid molarity. Only one titration was performed for each sample as obtaining the range of nitric acid molarity was more desirable than obtaining a precise determination for each sample.

For the development of a systematic method of sample preparation and data analysis, the major part of a mass dilution factor must be calculated. The mass dilution factor of a particular sample can be calculated using the following data points: the mass of the heroin sample, the mass of the undiluted solution, the volume of the undiluted sample, the volume of diluted sample, and the specific gravity of both the undiluted and diluted samples. However, it is not practical to determine acid molarity and specific gravity of 
more than 1,200 samples. Therefore, the same dilution was applied systematically to all samples and only the sample mass and the post-digestion sample masses were measured for each individual sample. Five replicate density measurements were taken from three sample replicates before and after dilution. The undiluted density was found to be $1.0817 \pm 0.0014 \mathrm{~g} / \mathrm{mL}$ and the diluted density was found to be $1.0358 \pm 0.0026 \mathrm{~g} / \mathrm{mL}$; these values were input into the mass dilution factor calculation. The equation for the calculation of the mass dilution factor applied to each sample is shown below in Equation 2. Using the mass dilution factor, the mass concentration of elements in solution $(\mu \mathrm{g} / \mathrm{L})$, from the instrumental analysis, was transformed into a mass concentration in the heroin samples $(\mu \mathrm{g} / \mathrm{kg})$.

Equation 2: Calculation of the mass dilution factor, shown in parentheses.

$$
\mu \mathrm{g} / \mathrm{L} \times\left(\frac{3.0 \mathrm{~mL}}{6.0 \mathrm{~mL}} \times \frac{1.082\left(\frac{\mathrm{g}}{\mathrm{ml}}\right) \text { undil. sample }}{1.036\left(\frac{\mathrm{g}}{\mathrm{ml}}\right) \text { dil. sample }} \times \frac{\mathrm{mg} \text { of sample }}{1000 *(\mathrm{~g} \text { of sample }-\mathrm{g} \text { of vial })}\right)^{-1}=\mu \mathrm{g} / \mathrm{kg}
$$

\subsection{Results}

\subsubsection{Data Analysis}

The method blank was subtracted from the rest of the diluted digest samples. Any measured concentration that was less than the LOD was assigned a value of the LOD for that element (i.e., LOD-filtering). A set of global LODs was calculated as the average LOD for each element over the 12 months of measurements. These were used for LODfiltering instead of the daily LODs to avoid introducing artificial differences to samples analyzed on different days. 
Up to this point all concentrations obtained were the concentrations of the solutions that were diluted for analysis by Q-ICP-MS and HR-ICP-MS. To determine the concentration in the original powdered sample the overall dilution factors were applied to LOD-filtered data. Applying the dilution factor is possible in the instrument software, but it is very time-consuming, and the data would not be properly LOD-filtered, therefore multiplying the filtered data by the dilution factor was done offline. As noted in subchapter 2.3.7, the overall dilution factors were calculated for each sample using the mass of powdered sample, the masses and densities of the $13 \mathrm{~mL}$ digest products, and subsequent dilution volumes.

The performance of the Q-ICP-MS and HR-ICP-MS methods was monitored by plotting the concentrations obtained for the spinach and heroin controls for each digestion on control charts using the Levey-Jennings Control Chart in JMP software (SAS Institute, USA). Routinely monitoring control charts facilitated identification of potential problems with either the digestion or the instrumental performance, see Figure 10-19. Each data point is a different digestion of the control sample, and the horizontal bars represent the number of standard deviations (SDs) from the mean (the green line): $\mathrm{C}$ is within $1 \mathrm{SD}, \mathrm{B}$ is within 2 SDs, and A is within 3 SDs. Any point that falls inside of 2 SDs is considered normal, between 2 SDs and 3 SDs should be investigated for potential problems and outside of 3 SDs is expected to be an erroneous data point and an outlier from a normally distributed set of data. Some examples are shown in the figures on the following pages for Q-ICP-MS data. 
Of note are the SRM NIST 1570a samples for digestions JD2-117, JD2-119, JD2120 are in the action zone for multiple elements, but not JD2-118, and since 117, 118 and 119 were analyzed on the same day this indicated that the problem was with the digestions, but not the analysis (see Figure 10-14). As mentioned previously, the total digestion of heroin samples was much easier than that of the SRM spinach leave matrix. One can see for the JD2-117, JD2-119 and JD2-120 digestions of the heroin duplicate samples, there were no apparent problems with the extractions (see Figure 15-19). As a precaution all samples in JD2-117, 118, 119 and 120 were re-digested and analyzed again.

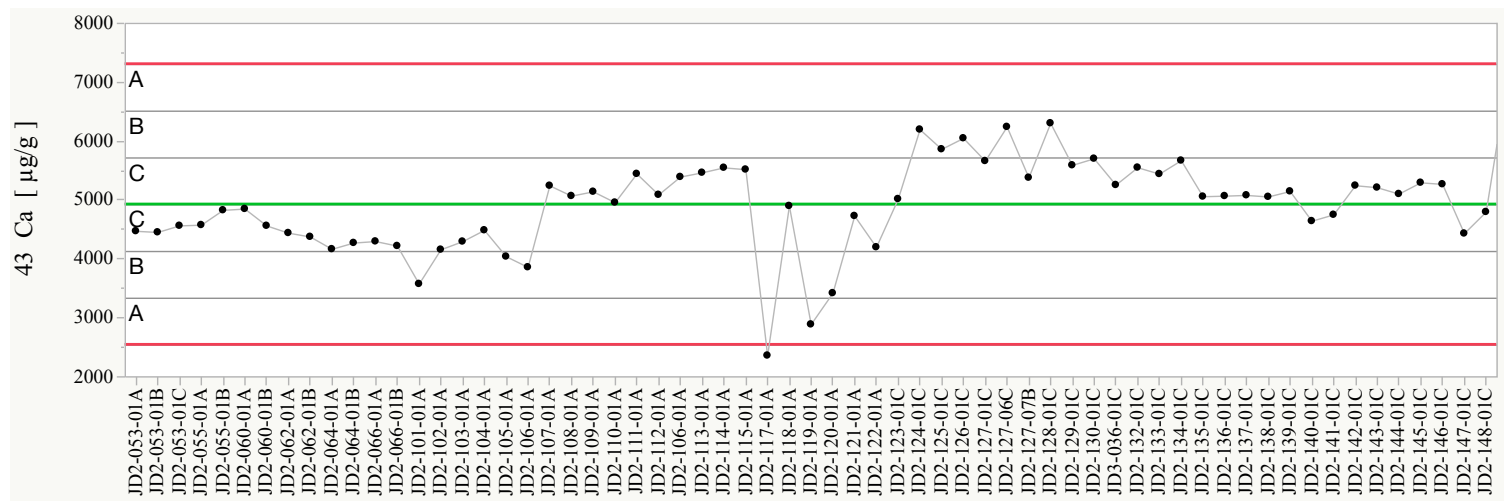

Figure 10. Levy-Jennings Control chart of ${ }^{43} \mathrm{Ca}$ in SRM NIST 1570a as measured by Q-ICP-MS.

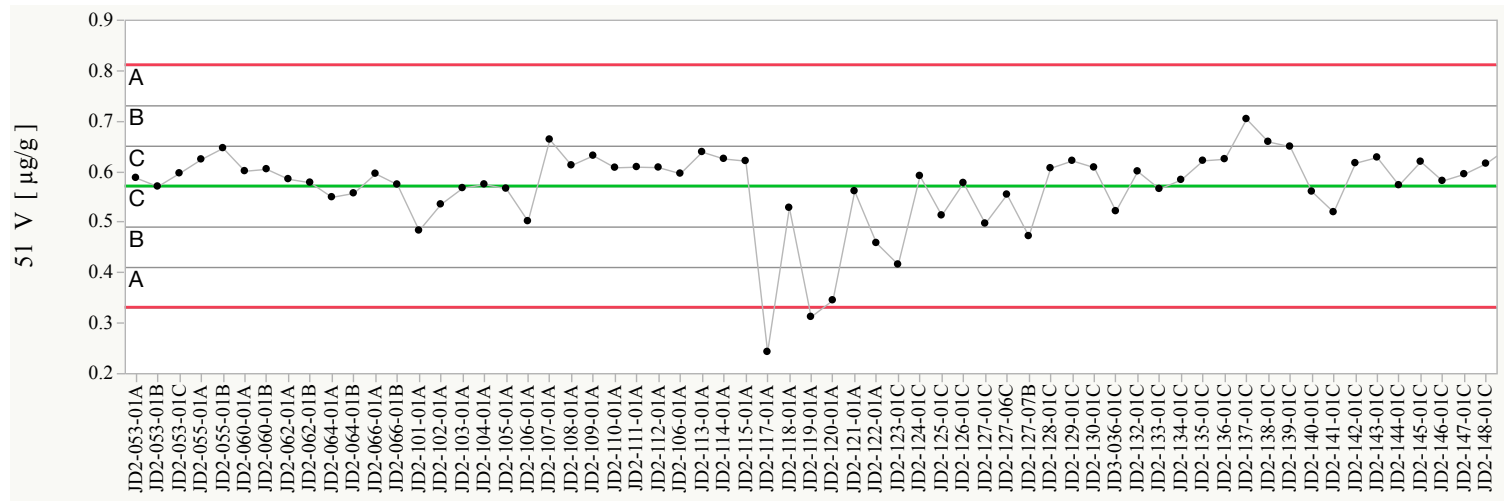

Figure 11. Levy-Jennings Control chart of ${ }^{51} \mathrm{~V}$ in SRM NIST 1570a as measured by Q-ICP-MS. 


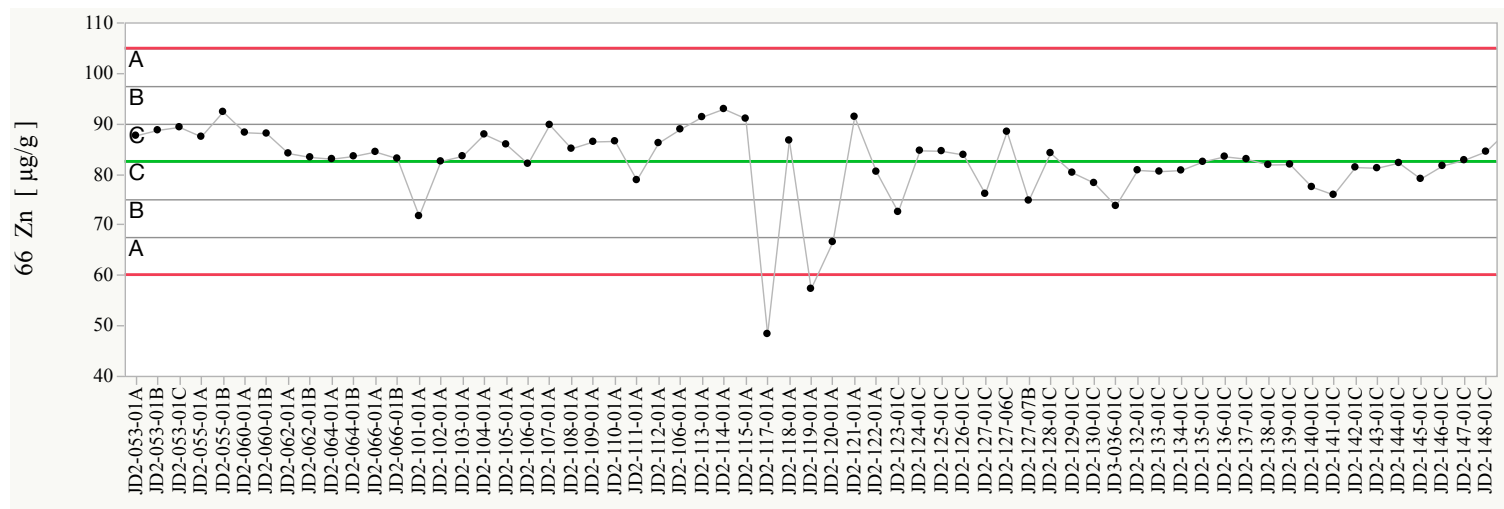

Figure 12. Levy-Jennings Control chart of ${ }^{66} \mathrm{Zn}$ in SRM NIST 1570a as measured by Q-ICP-MS.

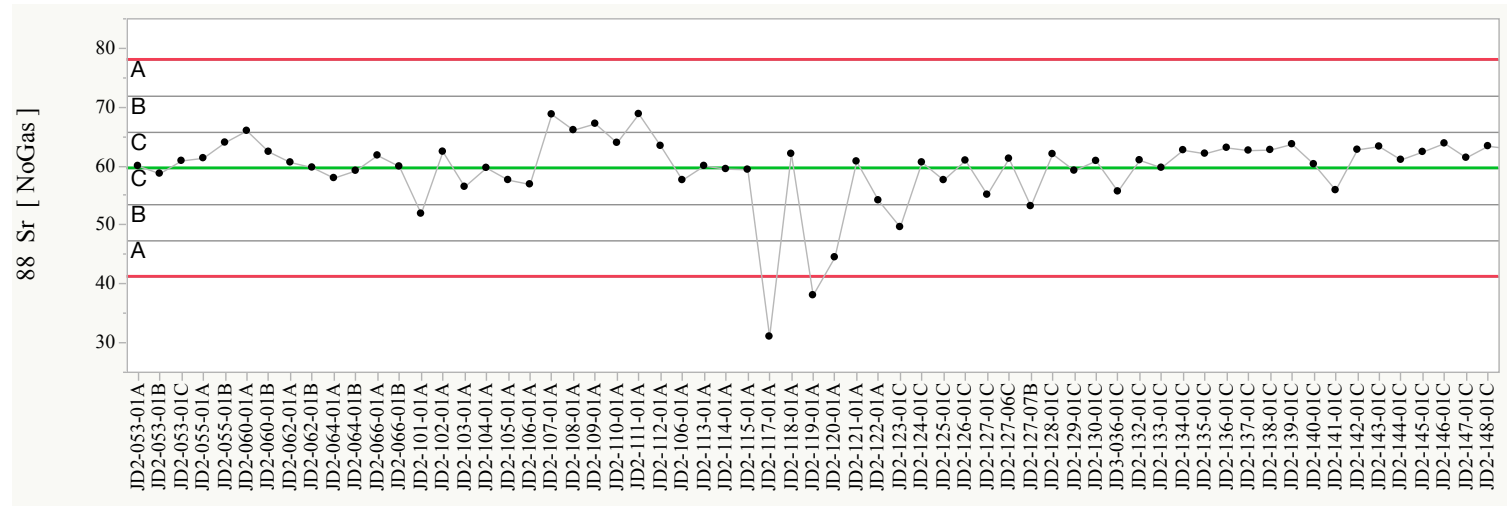

Figure 13. Levy-Jennings Control chart of ${ }^{88} \mathrm{Sr}$ in SRM NIST 1570a as measured by Q-ICP-MS.

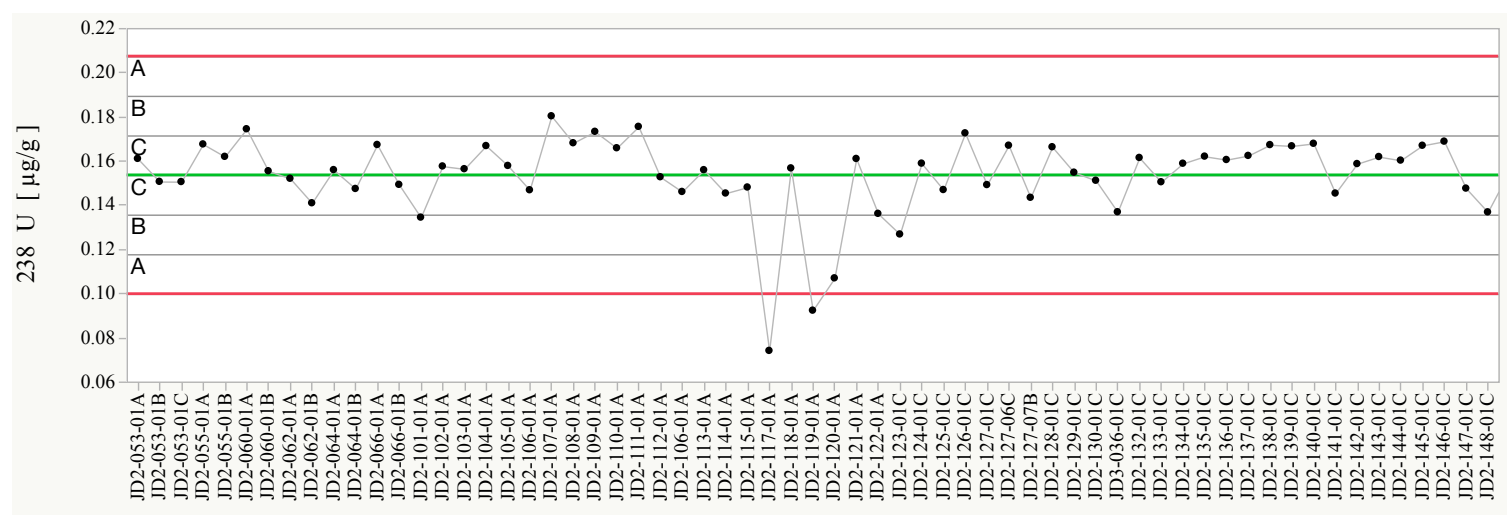

Figure 14. Levy-Jennings Control chart of ${ }^{238} \mathrm{U}$ in SRM NIST 1570a as measured by Q-ICP-MS. 


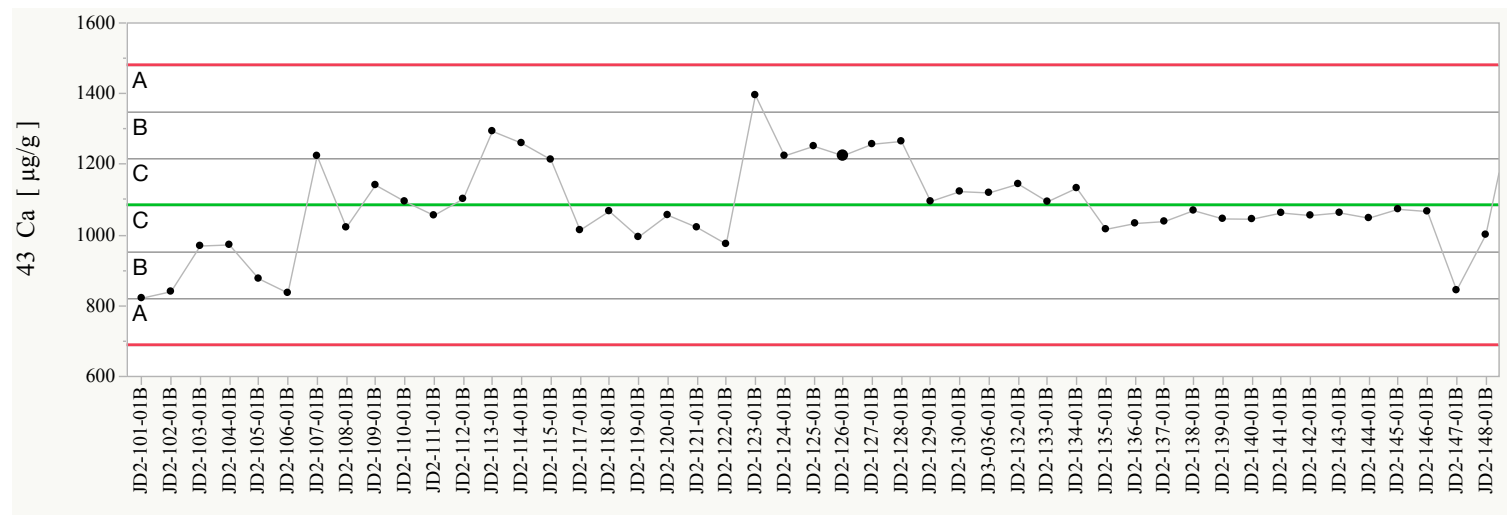

Figure 15. Levy-Jennings Control chart of ${ }^{43} \mathrm{Ca}$ in GFX2-83-8009/2 as measured by Q-ICP-MS.

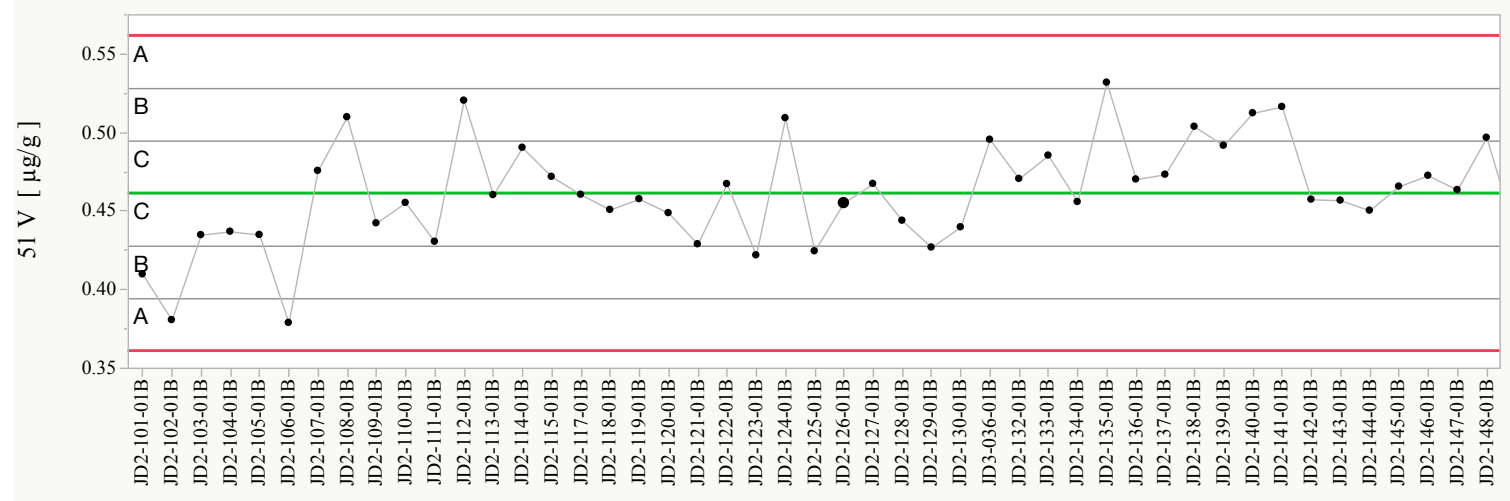

Figure 16. Levy-Jennings Control chart of ${ }^{51} \mathrm{~V}$ in GFX2-83-8009/2 as measured by Q-ICP-MS.

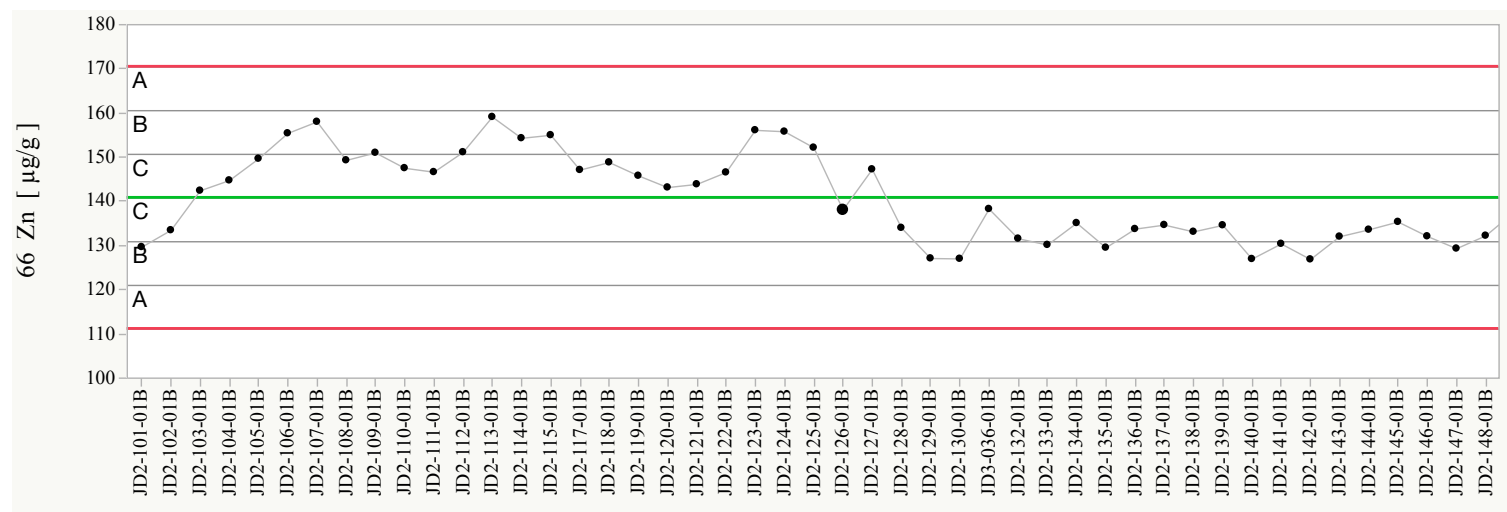

Figure 17. Levy-Jennings Control chart of ${ }^{66} \mathrm{Zn}$ in GFX2-83-8009/2 as measured by Q-ICP-MS. 


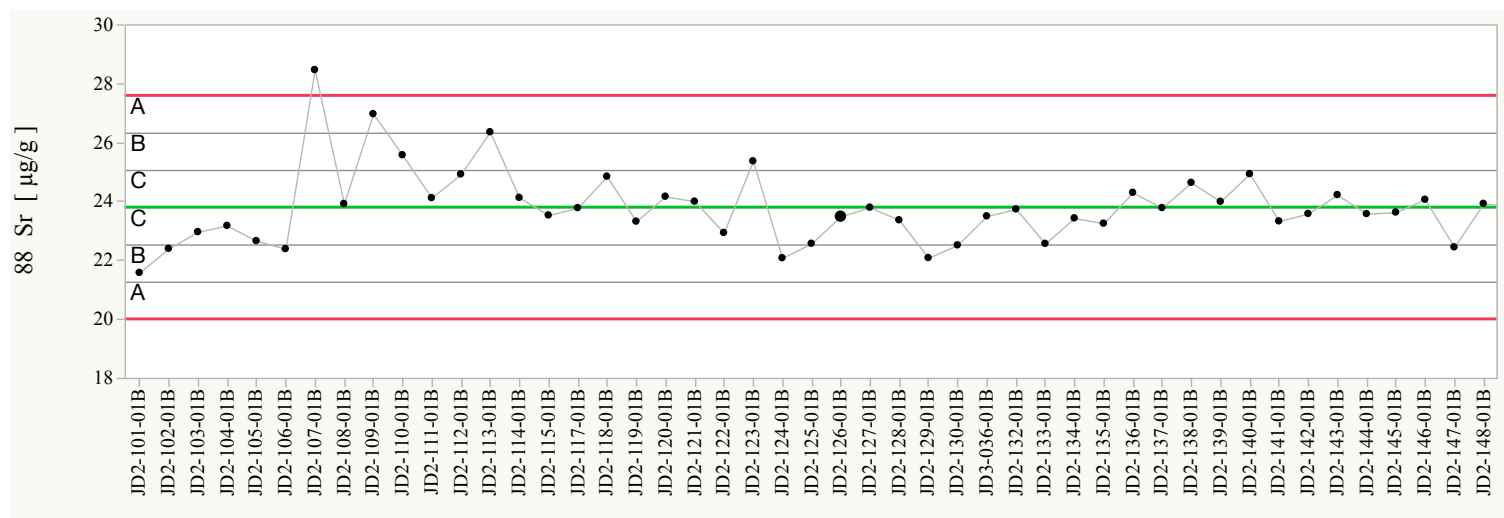

Figure 18. Levy-Jennings Control chart of ${ }^{88} \mathrm{Sr}$ in GFX2-83-8009/2 as measured by Q-ICP-MS.

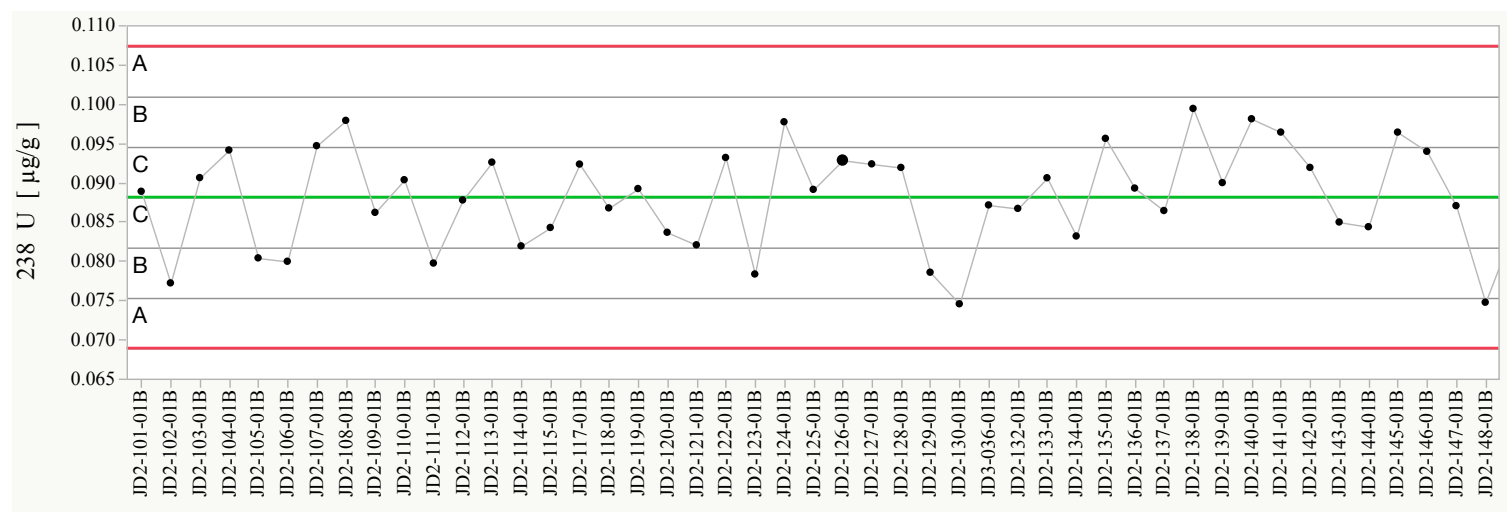

Figure 19. Levy-Jennings Control chart of ${ }^{238} \mathrm{U}$ in GFX2-83-8009/2 as measured by Q-ICP-MS.

As noted in the methods sections, many elements were measured by both Q-ICPMS and HR-ICP-MS to confirm that the results were reproducible. Examples are provided for $\mathrm{Cu}, \mathrm{Zn}, \mathrm{Sr}$ and $\mathrm{Ba}$ of the plot of the $\mu \mathrm{g} / \mathrm{g}$ concentration measured Q-ICP-MS concentrations on the x-axis and the measured HR-ICP-MS $\mu \mathrm{g} / \mathrm{g}$ concentration on the $\mathrm{y}$ axis. Pearson correlations coefficients were 0.9695 for $\mathrm{Cu}, 0.9912$ for $\mathrm{Zn}, 0.9950$ for $\mathrm{Sr}$ and 0.9947 for $\mathrm{Ba}$, the plot of the data is shown on Figure 20. The fact that the calibration solutions for both HR-ICP-MS as well as the Q-ICP-MS were prepared by serial dilution of a common stock contributed to the instrumental correlation. The elements $\mathrm{Cu}, \mathrm{Zn}, \mathrm{Sr}$ 
and $\mathrm{Ba}$ were chosen for this plot on the basis of having a suitable distribution of sample concentrations for an observable linearity, although they were not the only elements exhibiting good correlations. Other elements had large concentration disparity between the samples with low abundance and the samples with high abundance that linearity would be imperceptible. There were elements, such as $\mathrm{P}, \mathrm{Ar}$ and Se which were not well correlated between the two instruments, as a result of the analytical challenges of these analytes.
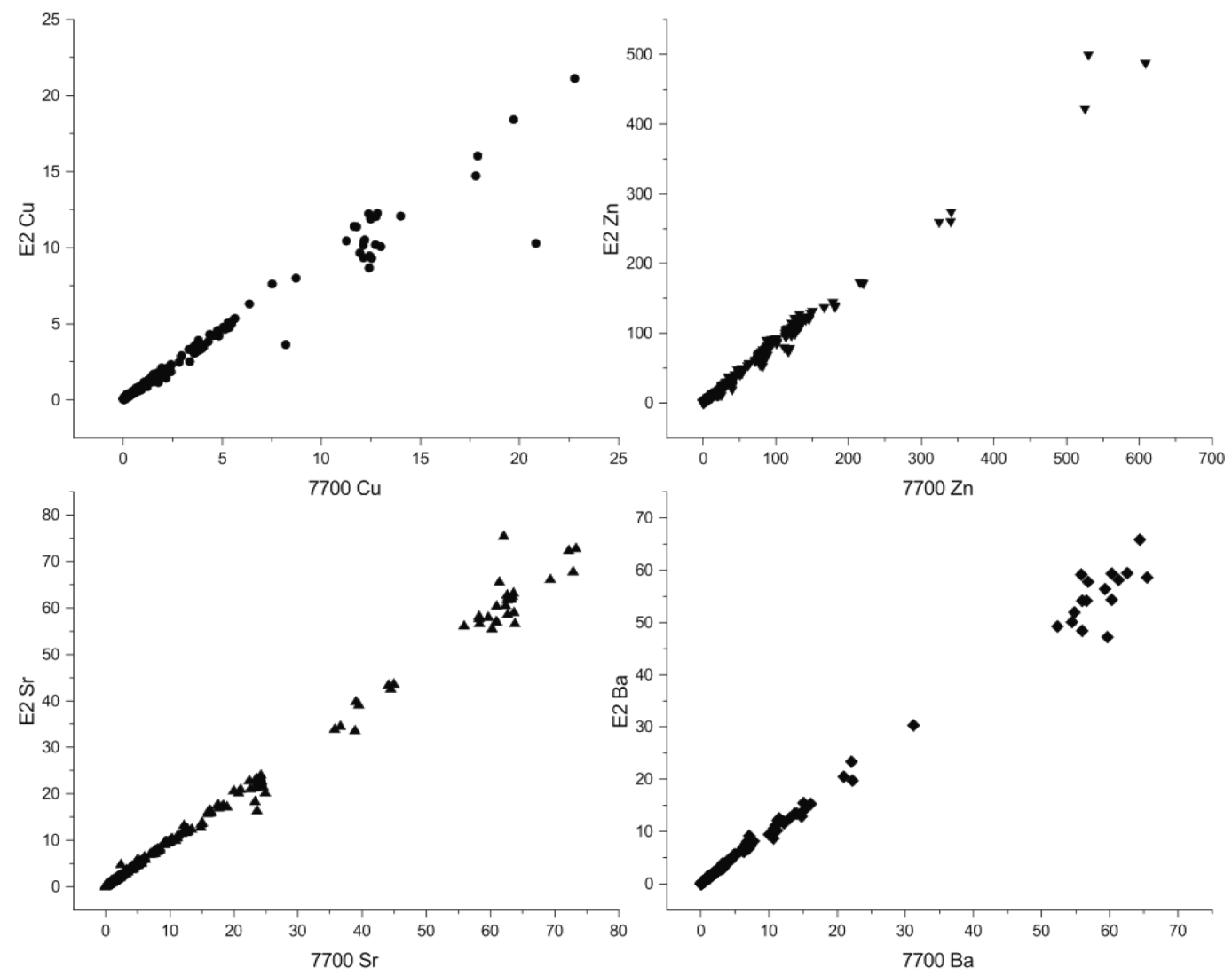

Figure 20. $\mathrm{x} / \mathrm{y}$ plot of $\mu \mathrm{g} / \mathrm{g}$ concentration heroin samples measured by the Agilent $7700 \mathrm{x}$ Q-ICP-MS on the $\mathrm{x}$-axis and the Thermo Fisher Element2 HR-ICP-MS on the y-axis for $\mathrm{Cu}, \mathrm{Zn}, \mathrm{Sr}$ and $\mathrm{Ba}$.

Some dispersion was seen at higher concentrations, which was likely because these concentrations were above the highest point in the calibration range of the HR-ICP-MS (30 
$\mu \mathrm{g} / \mathrm{g})$. The slopes of the lines are between 0.83 and 0.95 which suggests there is a systematic drift in the calculation of mass concentration in favor of a higher value in the Q-ICP-MS, which is also likely a result of a more concentrated upper-level calibration solution being used on the Q-ICP-MS of $100 \mu \mathrm{g} / \mathrm{g}$ as opposed to the HR-ICP-MS of 30 $\mu \mathrm{g} / \mathrm{g}$. However, the observation could also be a result of differences between the ionization efficiencies of the plasma or detector responsiveness in the Q-ICP-MS and HR-ICP-MS.

\subsubsection{Q-ICP-MS}

The analytical method of Q-ICP-MS of heroin samples prepared by micro-wave assisted acid digestion was validated by conducting repeated analyses of SRM NIST 1570a and the heroin duplicate control sample (GFX2-83-8002/9). The method limit of detection (LOD) was determined by multiplying the instrumental limit of detection, found by a linear calibration of 3 standard deviations of the $0 \mathrm{ppb}$ calibration solution, multiplied by the average mass dilution factor of SRM NIST 1570a and GFX2-83-8002/9. Since the overall dilution factor described above differed for each individual sample, an average dilution factor of 752 was used for the spinach control and 433 for the heroin control. The interday repeatability was found by determination of RSD over 62 separate preparations and analyses of the SRM NIST 1570a and 47 separate preparations and analyses of GFX2-83$8002 / 9$. The extraction recovery (Rec \%) was determined by the percent recovery of the calculated mass concentration of each listed element compared to the certified value on the certificate of analysis of SRM NIST 1570a; figures of merit are shown on Table 7. Since the GFX2-83-8002/9 does not have independent lab values Rec \% could not be calculated and therefore are not shown on Table 8 . 
Table 7. Figures of merit for analysis of the spinach control by Q-ICP-MS ( $\mathrm{n}=62$ ). Missing recovery values indicate elements not reported on the certificate of analysis of NIST SRM 1570a. An asterisk $(*)$ indicates elements were reported only as information values on the certificate of analysis of NIST SRM 1570a.

\begin{tabular}{|c|c|c|c|c|c|c|c|c|c|}
\hline & LOD & $\operatorname{MEAN}(\mu \mathrm{g} / \mathrm{g})$ & $\begin{array}{c}\text { RSD } \\
(\%)\end{array}$ & $\operatorname{Rec} \%$ & & LOD & MEAN $(\mu \mathrm{g} / \mathrm{g})$ & $\begin{array}{c}\text { RSD } \\
(\%)\end{array}$ & $\operatorname{Rec} \%$ \\
\hline${ }^{9} \mathrm{Be}$ & 0.002 & $0.004 \pm 0.0003$ & 33 & & ${ }^{118} \mathrm{Sn}$ & 0.007 & $0.033 \pm 0.0058$ & 70 & \\
\hline${ }^{11} \mathrm{~B}$ & 0.084 & $41 \pm 1.3$ & 13 & 107.7 & ${ }^{121} \mathrm{Sb}$ & 0.001 & $0.007 \pm 0.0008$ & 43 & \\
\hline${ }^{23} \mathrm{Na}$ & 4.2 & $19300 \pm 600$ & 10 & 105.8 & ${ }^{125} \mathrm{Te}$ & 0.006 & $0.008 \pm 0.0013$ & 62 & \\
\hline${ }^{24} \mathrm{Mg}$ & 0.032 & $9370 \pm 250$ & 10 & $104.1^{*}$ & ${ }^{133} \mathrm{Cs}$ & 0.001 & $0.04 \pm 0.011$ & 109 & \\
\hline${ }^{27} \mathrm{Al}$ & 0.18 & $214 \pm 8$ & 14 & 68.9 & ${ }^{137} \mathrm{Ba}$ & 0.006 & $6.8 \pm 0.19$ & 11 & \\
\hline${ }^{31} \mathrm{P}$ & 4.1 & $6020 \pm 260$ & 17 & 116.1 & ${ }^{139} \mathrm{La}$ & 0.0004 & $0.14 \pm 0.005$ & 14 & \\
\hline${ }^{39} \mathrm{~K}$ & 1.2 & $31100 \pm 800$ & 11 & 107.1 & ${ }^{140} \mathrm{Ce}$ & 0.0006 & $0.29 \pm 0.01$ & 13 & \\
\hline${ }^{43} \mathrm{Ca}$ & 0.4 & $4880 \pm 190$ & 15 & 32 & ${ }^{141} \mathrm{Pr}$ & 0.0001 & $0.033 \pm 0.0011$ & 14 & \\
\hline${ }^{47} \mathrm{Ti}$ & 0.08 & $14.9 \pm 0.7$ & 17 & & ${ }^{146} \mathrm{Nd}$ & 0.0001 & $0.12 \pm 0.004$ & 14 & \\
\hline${ }^{51} \mathrm{~V}$ & 0.002 & $0.57 \pm 0.02$ & 14 & 100.2 & ${ }^{147} \mathrm{Sm}$ & 0.0001 & $0.023 \pm 0.0008$ & 14 & \\
\hline${ }^{52} \mathrm{Cr}$ & 0.004 & $1.6 \pm 0.06$ & 15 & & ${ }^{153} \mathrm{Eu}$ & 0.0001 & $0.005 \pm 0.0002$ & 14 & 109.6 \\
\hline${ }^{55} \mathrm{Mn}$ & 0.003 & $82 \pm 2$ & 10 & 108.2 & ${ }^{157} \mathrm{Gd}$ & 0.0001 & $0.022 \pm 0.0008$ & 14 & \\
\hline${ }^{57} \mathrm{Fe}$ & 0.34 & $283 \pm 15$ & 21 & & ${ }^{163} \mathrm{Dy}$ & 0.00004 & $0.017 \pm 0.0006$ & 15 & \\
\hline${ }^{59} \mathrm{Co}$ & 0.001 & $0.37 \pm 0.01$ & 11 & 95.3 & ${ }^{165} \mathrm{Ho}$ & 0.00002 & $0.003 \pm 0.0001$ & 15 & \\
\hline${ }^{60} \mathrm{Ni}$ & 0.079 & $1.2 \pm 0.19$ & 65 & 54.4 & ${ }^{166} \mathrm{Er}$ & 0.00004 & $0.009 \pm 0.0003$ & 14 & \\
\hline${ }^{63} \mathrm{Cu}$ & 0.004 & $12 \pm 0.3$ & 9 & 98.6 & ${ }^{169} \mathrm{Tm}$ & 0.00001 & $0.001 \pm 0.0001$ & 19 & \\
\hline${ }^{66} \mathrm{Zn}$ & 0.086 & $82 \pm 1.9$ & 9 & 99.9 & ${ }^{172} \mathrm{Yb}$ & 0.0001 & $0.007 \pm 0.0003$ & 16 & \\
\hline${ }^{71} \mathrm{Ga}$ & 0.002 & $0.082 \pm 0.0028$ & 13 & & ${ }^{175} \mathrm{Lu}$ & 0.00004 & $0.001 \pm 0.0003$ & 111 & \\
\hline${ }^{75} \mathrm{As}$ & 0.02 & $0.04 \pm 0.0044$ & 44 & 58.1 & ${ }^{178} \mathrm{Hf}$ & 0.0001 & $0.004 \pm 0.0003$ & 27 & \\
\hline${ }^{78} \mathrm{Se}$ & 0.008 & $0.11 \pm 0.006$ & 20 & 96.9 & ${ }^{181} \mathrm{Ta}$ & 0.0005 & $0.0008 \pm 0.0001$ & 51 & \\
\hline${ }^{85} \mathrm{Rb}$ & 0.003 & $14 \pm 0.3$ & 8 & 109.7 & ${ }^{182} \mathrm{~W}$ & 0.003 & $0.008 \pm 0.0022$ & 103 & \\
\hline${ }^{88} \mathrm{Sr}$ & 0.002 & $60 \pm 1.6$ & 11 & 107.2 & ${ }^{197} \mathrm{Au}$ & 0.005 & $0.007 \pm 0.0015$ & 86 & \\
\hline${ }^{90} \mathrm{Zr}$ & 0.002 & $0.13 \pm 0.006$ & 19 & & ${ }^{205} \mathrm{Tl}$ & 0.003 & $0.018 \pm 0.0014$ & 31 & \\
\hline${ }^{93} \mathrm{Nb}$ & 0.0004 & $0.035 \pm 0.0017$ & 19 & & ${ }^{208} \mathrm{~Pb}$ & 0.002 & $0.18 \pm 0.012$ & 26 & $89^{*}$ \\
\hline${ }^{95} \mathrm{Mo}$ & 0.002 & $0.36 \pm 0.016$ & 17 & & ${ }^{232} \mathrm{Th}$ & 0.0002 & $0.043 \pm 0.0017$ & 16 & 90.5 \\
\hline${ }^{107} \mathrm{Ag}$ & 0.0006 & $0.016 \pm 0.004$ & 96 & & ${ }^{238} \mathrm{U}$ & 0.0002 & $0.15 \pm 0.005$ & 12 & 99.1 \\
\hline${ }^{111} \mathrm{Cd}$ & 0.003 & $2.8 \pm 0.07$ & 10 & 98.9 & & & & & \\
\hline
\end{tabular}

When prepared by microwave assisted acid digestion and analyzed by Q-ICP-MS, most elements above $0.5 \mathrm{ppm}$ which corresponds to $\sim 1 \mathrm{ppb}$ in the solution have RSD $<20 \%$ except for ${ }^{60} \mathrm{Ni}$, which experienced repeatability issues likely because of the high 
${ }^{60} \mathrm{Ni}$ background. As shown on Figure 21, beginning with digest JD2-117, ${ }^{60} \mathrm{Ni}$ present in the background caused the calculation of the mass concentration in the sample to drop. The ${ }^{60} \mathrm{Ni}$ signal was likely present by being introduced by the sampler and/or skimmer cones of the Q-ICP-MS as the background was not observed in the control charts of the HR-ICPMS. Once they were cleaned the background improved somewhat, and once they were replaced, the background returned to normal.

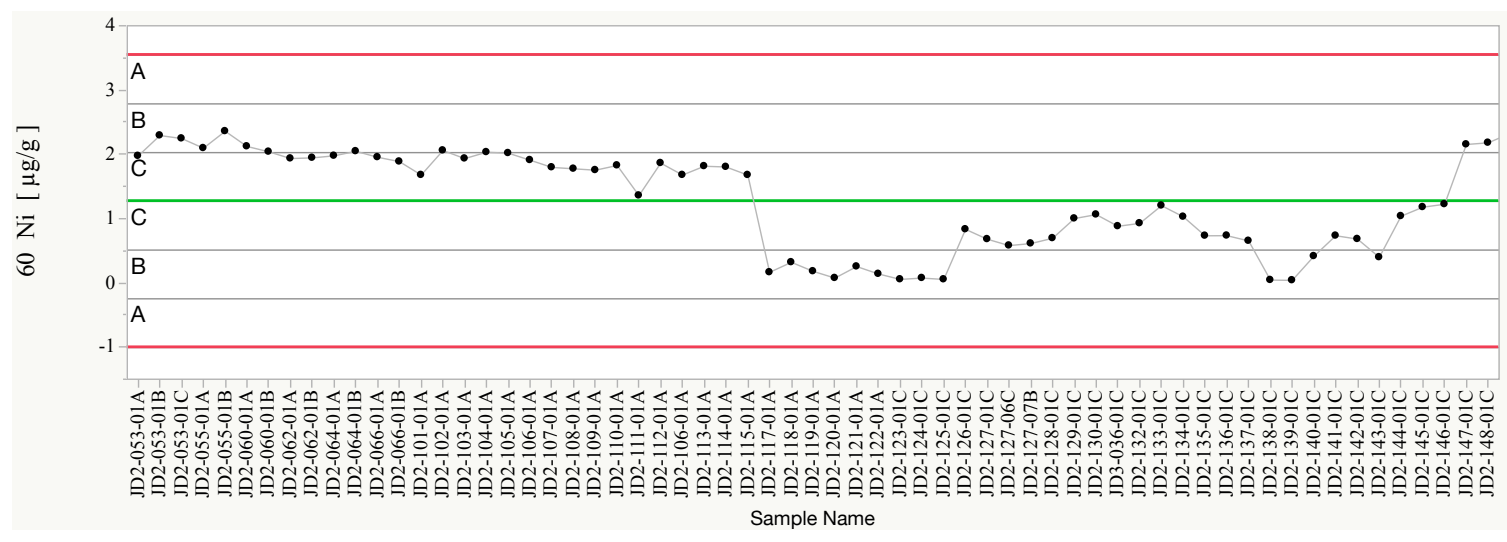

Figure 21. Levy-Jennings Control Chart of 60Ni for SRM NIST 1570a

The percent recoveries are within $\pm 10 \%$ of the certified values for all elements except for ${ }^{27} \mathrm{Al},{ }^{31} \mathrm{P},{ }^{43} \mathrm{Ca},{ }^{60} \mathrm{Ni}$ and ${ }^{75} \mathrm{As}$. The RSD for ${ }^{60} \mathrm{Ni}$ and ${ }^{43} \mathrm{Ca}$ are both quite low, which suggests there is some systematic inefficiency in attaining $100 \%$ extraction of that analyte. Perhaps this is a consequence of some fraction of $\mathrm{Al}$ and $\mathrm{Ni}$ being occluded with a siliceous mineral that is not dissolved without the inclusion of hydrofluoric acid. The isotopes of ${ }^{31} \mathrm{P}$ and ${ }^{75} \mathrm{As}$ suffer from well-known polyatomic interferences, ${ }^{14} \mathrm{~N}^{16} \mathrm{O}{ }^{1} \mathrm{H}$ and ${ }^{40} \mathrm{Ar}^{35} \mathrm{Cl}$ respectively. Nitrogen hydroxide is unavoidable as the digestion acid is $\mathrm{HNO}_{3}$ as well as nitrogen being part of the chemical structure of heroin. Argon chloride is also prevalent because of the vary large quantity of argon gas used in ICP-MS analysis as well 
as the heroin sample being of the $\mathrm{HCl}$ salt form for most of the samples. While the method was designed to remove the ${ }^{40} \mathrm{Ar}{ }^{35} \mathrm{Cl}$ interference for the analysis of ${ }^{75} \mathrm{As}$, the low concentration in both the SRM and heroin control and therefore validated performed was poor. As previously mentioned, ${ }^{60} \mathrm{Ni}$ suffered from a loss in measured concentration for many samples because of background contamination, which explains why the average percent recovery approximately half of what was expected.

Table 8. Figures of merit for analysis of the heroin control by Q-ICP-MS $(n=47)$.

\begin{tabular}{|c|c|c|c|c|c|c|c|}
\hline Isotope & LOD & MEAN $(\mu \mathrm{g} / \mathrm{g})$ & RSD (\%) & Isotope & LOD & MEAN $(\mu \mathrm{g} / \mathrm{g})$ & RSD (\%) \\
\hline${ }^{9} \mathrm{Be}$ & 0.001 & $0.003 \pm 0.0002$ & 24 & ${ }^{118} \mathrm{Sn}$ & 0.004 & $26.4 \pm 0.55$ & 7 \\
\hline${ }^{11} \mathrm{~B}$ & 0.05 & $0.48 \pm 0.186$ & 132 & ${ }^{121} \mathrm{Sb}$ & 0.001 & $0.021 \pm 0.0028$ & 46 \\
\hline${ }^{23} \mathrm{Na}$ & 2.4 & $720 \pm 14.3$ & 7 & ${ }^{125} \mathrm{Te}$ & 0.003 & $0.004 \pm 0.0005$ & 43 \\
\hline${ }^{24} \mathrm{Mg}$ & 0.02 & $85.2 \pm 3.52$ & 14 & ${ }^{133} \mathrm{Cs}$ & 0.0008 & $0.018 \pm 0.0006$ & 12 \\
\hline${ }^{27} \mathrm{Al}$ & 0.1 & $141 \pm 3.5$ & 8 & ${ }^{137} \mathrm{Ba}$ & 0.003 & $59.2 \pm 1.27$ & 7 \\
\hline${ }^{31} \mathrm{P}$ & 2.3 & $27.3 \pm 3$ & 37 & ${ }^{139} \mathrm{La}$ & 0.0002 & $0.17 \pm 0.024$ & 48 \\
\hline${ }^{39} \mathrm{~K}$ & 0.68 & $62.4 \pm 12.75$ & 70 & ${ }^{140} \mathrm{Ce}$ & 0.0004 & $0.27 \pm 0.05$ & 64 \\
\hline${ }^{43} \mathrm{Ca}$ & 0.25 & $1080 \pm 40$ & 12 & ${ }^{141} \operatorname{Pr}$ & 0.0001 & $0.033 \pm 0.0054$ & 55 \\
\hline${ }^{47} \mathrm{Ti}$ & 0.04 & $6.13 \pm 0.306$ & 17 & ${ }^{146} \mathrm{Nd}$ & 0.0001 & $0.12 \pm 0.016$ & 46 \\
\hline${ }^{51} \mathrm{~V}$ & 0.001 & $0.462 \pm 0.0099$ & 7 & ${ }^{147} \mathrm{Sm}$ & 0.0001 & $0.019 \pm 0.0009$ & 16 \\
\hline${ }^{52} \mathrm{Cr}$ & 0.002 & $1.15 \pm 0.066$ & 20 & ${ }^{153} \mathrm{Eu}$ & 0.00003 & $0.014 \pm 0.0008$ & 19 \\
\hline${ }^{55} \mathrm{Mn}$ & 0.002 & $3.76 \pm 0.325$ & 29 & ${ }^{157} \mathrm{Gd}$ & 0.0001 & $0.018 \pm 0.0008$ & 16 \\
\hline${ }^{57} \mathrm{Fe}$ & 0.19 & $189 \pm 12$ & 22 & ${ }^{163}$ Dy & 0.00002 & $0.012 \pm 0.0006$ & 16 \\
\hline${ }^{59} \mathrm{Co}$ & 0.001 & $0.059 \pm 0.0038$ & 22 & ${ }^{165} \mathrm{Ho}$ & 0.00001 & $0.002 \pm 0.0001$ & 19 \\
\hline${ }^{60} \mathrm{Ni}$ & 0.046 & $0.13 \pm 0.037$ & 99 & ${ }^{166} \mathrm{Er}$ & 0.00002 & $0.006 \pm 0.0004$ & 24 \\
\hline${ }^{63} \mathrm{Cu}$ & 0.002 & $4.2 \pm 0.64$ & 52 & ${ }^{169} \mathrm{Tm}$ & 0.00001 & $0.001 \pm 0.0001$ & 24 \\
\hline${ }^{66} \mathrm{Zn}$ & 0.05 & $141 \pm 3.1$ & 7 & ${ }^{172} \mathrm{Yb}$ & 0.0001 & $0.005 \pm 0.0003$ & 25 \\
\hline${ }^{71} \mathrm{Ga}$ & 0.001 & $0.075 \pm 0.0057$ & 26 & ${ }^{175} \mathrm{Lu}$ & 0.00002 & $0.001 \pm 0.0003$ & 132 \\
\hline${ }^{75} \mathrm{As}$ & 0.013 & $0.021 \pm 0.0027$ & 45 & ${ }^{178} \mathrm{Hf}$ & 0.0001 & $0.003 \pm 0.0001$ & 15 \\
\hline${ }^{78} \mathrm{Se}$ & 0.005 & $0.024 \pm 0.0016$ & 22 & ${ }^{181} \mathrm{Ta}$ & 0.0003 & $0.0004 \pm 0.0001$ & 62 \\
\hline${ }^{85} \mathrm{Rb}$ & 0.002 & $0.25 \pm 0.007$ & 10 & ${ }^{182} \mathrm{~W}$ & 0.002 & $0.013 \pm 0.0085$ & 219 \\
\hline${ }^{88} \mathrm{Sr}$ & 0.001 & $23.8 \pm 0.38$ & 5 & ${ }^{197} \mathrm{Au}$ & 0.00309 & $0.007 \pm 0.0017$ & 88 \\
\hline${ }^{90} \mathrm{Zr}$ & 0.001 & $0.12 \pm 0.01$ & 30 & ${ }^{205} \mathrm{Tl}$ & 0.002 & $0.003 \pm 0.0004$ & 51 \\
\hline${ }^{93} \mathrm{Nb}$ & 0.0002 & $0.022 \pm 0.0014$ & 22 & ${ }^{208} \mathrm{~Pb}$ & 0.001 & $2.43 \pm 0.248$ & 35 \\
\hline${ }^{95} \mathrm{Mo}$ & 0.001 & $0.098 \pm 0.0102$ & 36 & ${ }^{232} \mathrm{Th}$ & 0.0001 & $0.032 \pm 0.0028$ & 31 \\
\hline${ }^{107} \mathrm{Ag}$ & 0.0003 & $0.004 \pm 0.0007$ & 63 & ${ }^{238} \mathrm{U}$ & 0.0001 & $0.088 \pm 0.0019$ & 7 \\
\hline${ }^{111} \mathrm{Cd}$ & 0.001 & $0.075 \pm 0.002$ & 9 & & & & \\
\hline
\end{tabular}




\subsubsection{HR-ICP-MS}

The validation of the HR-ICP-MS method for heroin samples prepared by microwave assisted acid digestion the same as that of the Q-ICP-MS, which accomplished by means of repeated analyses of SRM NIST 1570a and the heroin duplicate control sample (GFX2-83-8002/9). The HR-ICP-MS did not perform as consistently for most major elements compared to the Q-ICP-MS. For most elements, the measured mean values from both instruments are within the reported uncertainty ranges, however the RSD's for many elements are much higher on the HR-ICP-MS. The figures of merit are given in Table 9 and Table 10 for the HR-ICP-MS. The mean, $95 \%$ confidence interval (CI), and inter-day relative standard deviation (RSD) were calculated from all $(n=61)$ analyses of each control.

The primary reasons for high inter-day RSDs for the Q-ICP-MS were low concentration of analyte in the sample or high concentrations in method blank. For elements such as $\mathrm{B}$ and $\mathrm{Ni}$, it was likely the low sample concentrations relative to the background signal of these elements that led to low reproducibility. For some elements, such as $\mathrm{B}, \mathrm{Na}, \mathrm{Al}, \mathrm{P}, \mathrm{S}, \mathrm{K}, \mathrm{Ca}$, the concentrations in the spinach control were above the upper calibration limit for the HR-ICP-MS $(30 \mu \mathrm{g} / \mathrm{g})$, resulting in poor precision and recovery (see Table 9). Low concentration elements, such as Lu and $\mathrm{W}$ in the spinach control (see Table 9) and Te, Lu and W in the heroin control (see Table 10), were very close to the LOD, which commonly results in poor RSD. The poor performance of As and Se by the HR-ICP-MS was because of a problem with the mass calibration in HR for a few

of the analysis days, which allowed the interfering polyatomic ions of $\left({ }^{40} \mathrm{Ar}{ }^{35} \mathrm{Cl}\right)^{+}$and 
$\left({ }^{40} \mathrm{Ar}{ }^{38} \mathrm{Ar}\right)^{+}$to enter the analytical window, resulting in much greater calculated recovery than was expected from the SRM NIST 1570a.

Table 9. Figures of merit for analysis of the spinach control by HR-ICP-MS ( $\mathrm{n}=61$ ). Missing recovery values indicate elements not reported on the certificate of analysis of NIST SRM 1570a. An asterisk $\left.{ }^{*}\right)$ indicates elements were reported only as information values on the certificate of analysis of NIST SRM 1570a.

\begin{tabular}{|c|c|c|c|c|c|c|c|c|c|}
\hline Isotope & LOD & $\begin{array}{c}\text { MEAN } \\
(\mu \mathrm{g} / \mathrm{g})\end{array}$ & $\begin{array}{c}\text { RSD } \\
(\%)\end{array}$ & $\operatorname{Rec} \%$ & Isotope & LOD & MEAN $(\mu \mathrm{g} / \mathrm{g})$ & $\begin{array}{c}\text { RSD } \\
(\%)\end{array}$ & $\begin{array}{c}\operatorname{Rec} \\
\% \\
\end{array}$ \\
\hline${ }^{9} \mathrm{Be}$ & 0.0051 & $0.041 \pm 0.014$ & 141 & & ${ }^{95} \mathrm{Mo}$ & 0.0018 & $0.45 \pm 0.02$ & 16 & \\
\hline${ }^{11} \mathrm{~B}$ & 0.059 & $46 \pm 2$ & 18 & 123 & ${ }^{107} \mathrm{Ag}$ & 0.0011 & $0.024 \pm 0.003$ & 50 & \\
\hline${ }^{23} \mathrm{Na}$ & 5.2 & $1190 \pm 820$ & 275 & 7 & ${ }^{111} \mathrm{Cd}$ & 0.0008 & $2.8 \pm 0.1$ & 11 & 97 \\
\hline${ }^{24} \mathrm{Mg}$ & 0.084 & $8660 \pm 360$ & 17 & $96^{*}$ & ${ }^{118} \mathrm{Sn}$ & 0.0058 & $0.071 \pm 0.019$ & 109 & \\
\hline${ }^{27} \mathrm{Al}$ & 0.076 & $205 \pm 8$ & 15 & 66 & ${ }^{121} \mathrm{Sb}$ & 0.0005 & $0.019 \pm 0.004$ & 92 & \\
\hline${ }^{31} \mathrm{P}$ & 0.11 & $5550 \pm 320$ & 23 & 107 & ${ }^{125} \mathrm{Te}$ & 0.0029 & $0.18 \pm 0.07$ & 148 & \\
\hline${ }^{32} \mathrm{~S}$ & 0.81 & $6060 \pm 430$ & 28 & 121 & ${ }^{133} \mathrm{Cs}$ & 0.0003 & $0.028 \pm 0.005$ & 76 & \\
\hline${ }^{39} \mathrm{~K}$ & 0.049 & $26800 \pm 2400$ & 36 & 92 & ${ }^{137} \mathrm{Ba}$ & 0.0026 & $7.1 \pm 0.3$ & 14 & \\
\hline${ }^{43} \mathrm{Ca}$ & 0.26 & $3920 \pm 690$ & 70 & 26 & ${ }^{139} \mathrm{La}$ & 0.0003 & $0.15 \pm 0.01$ & 13 & \\
\hline${ }^{44} \mathrm{Ca}$ & 0.02 & $3990 \pm 700$ & 70 & 26 & ${ }^{140} \mathrm{Ce}$ & 0.0003 & $0.3 \pm 0.01$ & 12 & \\
\hline${ }^{47} \mathrm{Ti}$ & 0.016 & $16 \pm 1$ & 19 & & ${ }^{141} \operatorname{Pr}$ & 0.00009 & $0.036 \pm 0.001$ & 16 & \\
\hline${ }^{51} \mathrm{~V}$ & 0.0007 & $0.62 \pm 0.02$ & 11 & 110 & ${ }^{146} \mathrm{Nd}$ & 0.00036 & $0.14 \pm 0.01$ & 18 & \\
\hline${ }^{52} \mathrm{Cr}$ & 0.0035 & $1.7 \pm 0.1$ & 14 & & ${ }^{147} \mathrm{Sm}$ & 0.00018 & $0.036 \pm 0.005$ & 51 & \\
\hline${ }^{55} \mathrm{Mn}$ & 0.0032 & $72 \pm 2$ & 14 & 95 & ${ }^{153} \mathrm{Eu}$ & 0.00016 & $0.011 \pm 0.001$ & 47 & 191 \\
\hline${ }^{56} \mathrm{Fe}$ & 0.037 & $242 \pm 10$ & 16 & & ${ }^{157} \mathrm{Gd}$ & 0.00039 & $0.035 \pm 0.004$ & 43 & \\
\hline${ }^{59} \mathrm{Co}$ & 0.0028 & $0.41 \pm 0.01$ & 12 & 104 & ${ }^{163} \mathrm{Dy}$ & 0.00018 & $0.024 \pm 0.003$ & 41 & \\
\hline${ }^{60} \mathrm{Ni}$ & 0.0035 & $2 \pm 0.1$ & 18 & 95 & ${ }^{165} \mathrm{Ho}$ & 0.00004 & $0.0051 \pm 0.0006$ & 49 & \\
\hline${ }^{63} \mathrm{Cu}$ & 0.0078 & $11 \pm 0$ & 14 & 93 & ${ }^{166} \mathrm{Er}$ & 0.0001 & $0.015 \pm 0.002$ & 49 & \\
\hline${ }^{66} \mathrm{Zn}$ & 0.11 & $75 \pm 4$ & 22 & 92 & ${ }^{169} \mathrm{Tm}$ & 0.00004 & $0.0028 \pm 0.0006$ & 84 & \\
\hline${ }^{69} \mathrm{Ga}$ & 0.0006 & $0.11 \pm 0.02$ & 79 & & ${ }^{172} \mathrm{Yb}$ & 0.00014 & $0.015 \pm 0.003$ & 69 & \\
\hline${ }^{72} \mathrm{Ge}$ & 0.0023 & $0.67 \pm 0.27$ & 159 & & ${ }^{175} \mathrm{Lu}$ & 0.0018 & $0.0076 \pm 0.0043$ & 226 & \\
\hline${ }^{75} \mathrm{As}$ & 0.012 & $1.3 \pm 0.5$ & 154 & 1948 & ${ }^{178} \mathrm{Hf}$ & 0.00014 & $0.011 \pm 0.003$ & 90 & \\
\hline${ }^{77} \mathrm{Se}$ & 0.091 & $17 \pm 7$ & 160 & 14504 & ${ }^{182} \mathrm{~W}$ & 0.016 & $0.027 \pm 0.005$ & 80 & \\
\hline${ }^{85} \mathrm{Rb}$ & 0.001 & $14 \pm 0$ & 10 & 109 & ${ }^{205} \mathrm{Tl}$ & 0.00009 & $0.026 \pm 0.004$ & 54 & \\
\hline${ }^{88} \mathrm{Sr}$ & 0.017 & $59 \pm 1$ & 10 & 107 & ${ }^{208} \mathrm{~Pb}$ & 0.0009 & $0.2 \pm 0.03$ & 61 & $102 *$ \\
\hline${ }^{90} \mathrm{Zr}$ & 0.0008 & $0.14 \pm 0.01$ & 21 & & ${ }^{232} \mathrm{Th}$ & 0.00005 & $0.054 \pm 0.008$ & 63 & 112 \\
\hline${ }^{93} \mathrm{Nb}$ & 0.0005 & $0.04 \pm 0.002$ & 18 & & ${ }^{238} \mathrm{U}$ & 0.00005 & $0.18 \pm 0.03$ & 62 & 117 \\
\hline
\end{tabular}


Table 10. Figures of merit for analysis of the heroin control by HR-ICP-MS $(n=48)$.

\begin{tabular}{|c|c|c|c|c|c|c|c|}
\hline Isotope & LOD & MEAN $(\mu \mathrm{g} / \mathrm{g})$ & RSD (\%) & Isotope & LOD & MEAN $(\mu \mathrm{g} / \mathrm{g})$ & RSD (\%) \\
\hline${ }^{9} \mathrm{Be}$ & 0.003 & $0.0046 \pm 0.0009$ & 73 & ${ }^{95} \mathrm{Mo}$ & 0.001 & $0.099 \pm 0.009$ & 33 \\
\hline${ }^{11} \mathrm{~B}$ & 0.034 & $0.38 \pm 0.13$ & 118 & ${ }^{107} \mathrm{Ag}$ & 0.00063 & $0.0056 \pm 0.0007$ & 47 \\
\hline${ }^{23} \mathrm{Na}$ & 3 & $657 \pm 40$ & 22 & ${ }^{111} \mathrm{Cd}$ & 0.00048 & $0.069 \pm 0.002$ & 11 \\
\hline${ }^{24} \mathrm{Mg}$ & 0.048 & $80 \pm 4$ & 19 & ${ }^{118} \mathrm{Sn}$ & 0.0033 & $24 \pm 1$ & 9 \\
\hline${ }^{27} \mathrm{Al}$ & 0.044 & $130 \pm 4$ & 10 & ${ }^{121} \mathrm{Sb}$ & 0.00027 & $0.02 \pm 0.002$ & 45 \\
\hline${ }^{31} \mathrm{P}$ & 0.064 & $34 \pm 3$ & 27 & ${ }^{125} \mathrm{Te}$ & 0.0017 & $0.0044 \pm 0.0024$ & 193 \\
\hline${ }^{32} \mathrm{~S}$ & 0.46 & $338 \pm 16$ & 17 & ${ }^{133} \mathrm{Cs}$ & 0.00019 & $0.017 \pm 0.001$ & 19 \\
\hline${ }^{39} \mathrm{~K}$ & 0.028 & $52 \pm 8$ & 56 & ${ }^{137} \mathrm{Ba}$ & 0.0015 & $55 \pm 2$ & 11 \\
\hline${ }^{43} \mathrm{Ca}$ & 0.15 & $1060 \pm 110$ & 38 & ${ }^{139} \mathrm{La}$ & 0.00017 & $0.18 \pm 0.03$ & 54 \\
\hline${ }^{44} \mathrm{Ca}$ & 0.011 & $1090 \pm 110$ & 36 & ${ }^{140} \mathrm{Ce}$ & 0.00016 & $0.26 \pm 0.05$ & 70 \\
\hline${ }^{47} \mathrm{Ti}$ & 0.0093 & $5.7 \pm 0.2$ & 15 & ${ }^{141} \operatorname{Pr}$ & 0.00005 & $0.033 \pm 0.007$ & 71 \\
\hline${ }^{51} \mathrm{~V}$ & 0.00038 & $0.44 \pm 0.01$ & 7 & ${ }^{146} \mathrm{Nd}$ & 0.00021 & $0.12 \pm 0.02$ & 56 \\
\hline${ }^{52} \mathrm{Cr}$ & 0.002 & $1.1 \pm 0.1$ & 18 & ${ }^{147} \mathrm{Sm}$ & 0.0001 & $0.019 \pm 0.001$ & 17 \\
\hline${ }^{55} \mathrm{Mn}$ & 0.0019 & $3.3 \pm 0.1$ & 11 & ${ }^{153} \mathrm{Eu}$ & 0.00009 & $0.025 \pm 0.001$ & 16 \\
\hline${ }^{56} \mathrm{Fe}$ & 0.021 & $157 \pm 6$ & 14 & ${ }^{157} \mathrm{Gd}$ & 0.00023 & $0.022 \pm 0.002$ & 38 \\
\hline${ }^{59} \mathrm{Co}$ & 0.0016 & $0.058 \pm 0.005$ & 30 & ${ }^{163} \mathrm{Dy}$ & 0.0001 & $0.011 \pm 0.001$ & 15 \\
\hline${ }^{60} \mathrm{Ni}$ & 0.002 & $0.35 \pm 0.01$ & 11 & ${ }^{165} \mathrm{Ho}$ & 0.00002 & $0.0021 \pm 0.0001$ & 19 \\
\hline${ }^{63} \mathrm{Cu}$ & 0.0045 & $3.9 \pm 0.6$ & 52 & ${ }^{166} \mathrm{Er}$ & 0.00006 & $0.006 \pm 0.001$ & 18 \\
\hline${ }^{66} \mathrm{Zn}$ & 0.064 & $126 \pm 5$ & 14 & ${ }^{169} \mathrm{Tm}$ & 0.00002 & $0.00076 \pm 0.00007$ & 31 \\
\hline${ }^{69} \mathrm{Ga}$ & 0.00034 & $0.051 \pm 0.002$ & 11 & ${ }^{172} \mathrm{Yb}$ & 0.00008 & $0.0046 \pm 0.0003$ & 24 \\
\hline${ }^{72} \mathrm{Ge}$ & 0.0013 & $0.017 \pm 0.011$ & 227 & ${ }^{175} \mathrm{Lu}$ & 0.001 & $0.0021 \pm 0.0019$ & 310 \\
\hline${ }^{75} \mathrm{As}$ & 0.0069 & $0.048 \pm 0.017$ & 128 & ${ }^{178} \mathrm{Hf}$ & 0.00008 & $0.0029 \pm 0.0002$ & 20 \\
\hline${ }^{77} \mathrm{Se}$ & 0.052 & $0.35 \pm 0.23$ & 231 & ${ }^{182} \mathrm{~W}$ & 0.0092 & $0.026 \pm 0.013$ & 177 \\
\hline${ }^{85} \mathrm{Rb}$ & 0.00055 & $0.23 \pm 0.01$ & 15 & ${ }^{205} \mathrm{Tl}$ & 0.00005 & $0.0019 \pm 0.0001$ & 13 \\
\hline${ }^{88} \mathrm{Sr}$ & 0.01 & $22 \pm 1$ & 9 & ${ }^{208} \mathrm{~Pb}$ & 0.00051 & $2.4 \pm 0.2$ & 36 \\
\hline${ }^{90} \mathrm{Zr}$ & 0.00047 & $0.097 \pm 0.006$ & 21 & ${ }^{232} \mathrm{Th}$ & 0.00003 & $0.031 \pm 0.003$ & 31 \\
\hline${ }^{93} \mathrm{Nb}$ & 0.00026 & $0.02 \pm 0.001$ & 17 & ${ }^{238} \mathrm{U}$ & 0.00003 & $0.087 \pm 0.003$ & 12 \\
\hline
\end{tabular}

Relative to the results from the Q-ICP-MS the RSD are worse for the HR-ICP-MS. While unexpected, there are a few explanations for the better performance of the Q-ICPMS. The primarily cause was the author's inexperience in using the instrument and performance was much less easily monitored during analysis. The instrument software for 
the Q-ICP-MS, Masshunter 4.1 version C.01.01 (Agilent Technologies, USA) was much more amenable to method modification in response to observations made over the course of analysis the that of the HR-ICP-MS, Thermo ELEMENT 3.1.2.242 (Thermo Fisher, USA). The HR-ICP-MS was not initially equipped for an externally supplied calibration solution, and spiking of an internal standard solution was required for about half of the samples. Once it was set up, it introduced some challenges with proper sample stabilization time. Samples needed to be reanalyzed once the solution to the sample stabilization time was identified. Additionally, the Q-ICP-MS had a Peltier-chilled spray chamber which is designed to reduce the formation of oxide interferences, and typically oxides were approximately $2 \%$ on the Q-ICP-MS and $15 \%$ on the HR-ICP-MS. Lastly, because of the longer sampling time, the workflow was established to first analyze the samples on Q-ICPMS and then transfer them to the HR-ICP-MS. It is possible that there could have some external contamination during the analysis on Q-ICP-MS, during the transfer or whilst awaiting analysis on the HR-ICP-MS for the samples to cause the relatively poorer repeatability of the samples. Therefore, the HR-ICP-MS results were not used in subsequent statistical analysis.

\subsubsection{Method Performance with Heroin Samples}

An assessment of repeatability, and reproducibility was conducted for the heroin control sample and two other heroin samples. Evaluating RSD values for both intra-sample and inter-sample deviation helped to explain whether the variation is a result of instrumental challenges (primarily as a result of low sample concentrations) or a result of heterogeneity within the sample. It was recognized that homogenization would be useful 
to reduce sampling variation, however because of the sample is a Schedule 1 controlled substance, loss of sample, workplace contamination and security concerns made physical homogenization undesirable. Therefore, the sample was simply agitated in its vessel prior to sampling by gentle shaking. In addition, a clean spatula was used to stir the sample somewhat during sampling. Finally, in lieu of homogenization of the heroin samples, to mitigate bias from intra-sample variability, three separate replicates were taken for every sample with very few exceptions, and only when the sample mass was limited.

The ideal sample masses during routine sample preparation was $60-70 \mathrm{mg}$, however judging the sample size was difficult in some cases. The dry powder density was greatly variable sample to sample, therefore some sample masses were a low as $30 \mathrm{mg}$ while others as high as $100 \mathrm{mg}$. Sample was not returned to the parent vessel once removed to avoid contamination of the bulk samples, as the excess sample was to be returned to DEA-STRL following the conclusion of the research. To evaluate the reproducibility of the method using a large mass range, 8 samples of 3 different heroin samples were digested over a mass range of approximately $30 \mathrm{mg}$ to $120 \mathrm{mg}$. Table 11, Table 12 and Table 13 show the repeatability of the method, as relative standard deviation (RSD) of the calculated mass concentration in the sample $(\mu \mathrm{g} / \mathrm{g})$ and the reproducibility as a linear $(\mathrm{x} / \mathrm{y})$ function of the Pearson correlation coefficient $\left(\mathrm{R}^{2}\right)$ of the sample mass and the elemental concentration in the post-digest solutions. Only elements which met criteria of being repeatable, by having relative standard deviations less than $30 \%$, and reproducible with a Pearson correlation coefficient of greater than 0.7 are shown on the following tables. 
Table 11. Elements of GFX2-83-8002/9 with Repeatability ( $<30 \%$ RSD) and Reproducibility $\left(>0.7 \mathrm{R}^{2}\right) ; \mathrm{N}=8$

\begin{tabular}{|c|c|c|c|c|c|c|c|}
\hline Isotope & Mean $(\mu \mathrm{g} / \mathrm{g})$ & RSD & $\mathrm{R}^{2}$ & Isotope & Mean $(\mu \mathrm{g} / \mathrm{g})$ & RSD & $\mathrm{R}^{2}$ \\
\hline${ }^{9} \mathrm{Be}$ & $0.00507 \pm 0.00087$ & $13.9 \%$ & 0.85042 & ${ }^{107} \mathrm{Ag}$ & $0.00586 \pm 0.00116$ & $23.6 \%$ & 0.75477 \\
\hline${ }^{10} \mathrm{~B}$ & & & & ${ }^{111} \mathrm{Cd}$ & $0.0774 \pm 0.0084$ & $12.9 \%$ & 0.93542 \\
\hline${ }^{11} \mathrm{~B}$ & & & & ${ }^{118} \mathrm{Sn}$ & $26.4 \pm 1.3$ & $5.7 \%$ & 0.98454 \\
\hline${ }^{23} \mathrm{Na}$ & $695 \pm 21$ & $3.6 \%$ & 0.99475 & ${ }^{121} \mathrm{Sb}$ & $0.0191 \pm 0.0016$ & $10.0 \%$ & 0.9524 \\
\hline${ }^{24} \mathrm{Mg}$ & $83.6 \pm 11.3$ & $16.1 \%$ & 0.83941 & ${ }^{125} \mathrm{Te}$ & & & \\
\hline${ }^{27} \mathrm{Al}$ & $132 \pm 9$ & $8.3 \%$ & 0.97952 & ${ }^{133} \mathrm{Cs}$ & & & \\
\hline${ }^{31} \mathrm{P}$ & & & & ${ }^{137} \mathrm{Ba}$ & $56.1 \pm 3.3$ & $7.1 \%$ & 0.96142 \\
\hline${ }^{39} \mathrm{~K}$ & & & & ${ }^{139} \mathrm{La}$ & $0.154 \pm 0.019$ & $14.5 \%$ & 0.89193 \\
\hline${ }^{43} \mathrm{Ca}$ & $955 \pm 40$ & $5.0 \%$ & 0.99604 & ${ }^{140} \mathrm{Ce}$ & $0.228 \pm 0.041$ & $21.4 \%$ & 0.80435 \\
\hline${ }^{47} \mathrm{Ti}$ & $6.45 \pm 0.83$ & $15.4 \%$ & 0.92668 & ${ }^{141} \operatorname{Pr}$ & $0.0294 \pm 0.0047$ & $19.3 \%$ & 0.79589 \\
\hline${ }^{51} \mathrm{~V}$ & $0.417 \pm 0.019$ & $5.5 \%$ & 0.98701 & ${ }^{146} \mathrm{Nd}$ & $0.0988 \pm 0.0178$ & $21.5 \%$ & 0.80764 \\
\hline${ }^{52} \mathrm{Cr}$ & & & & ${ }^{147} \mathrm{Sm}$ & $0.0187 \pm 0.0033$ & $21.1 \%$ & 0.78888 \\
\hline${ }^{55} \mathrm{Mn}$ & $3.32 \pm 0.16$ & $5.8 \%$ & 0.98566 & ${ }^{153} \mathrm{Eu}$ & $0.016 \pm 0.0011$ & $8.0 \%$ & 0.93965 \\
\hline${ }^{57} \mathrm{Fe}$ & $163 \pm 8$ & $5.5 \%$ & 0.98303 & ${ }^{157} \mathrm{Gd}$ & $0.0173 \pm 0.0025$ & $17.2 \%$ & 0.84345 \\
\hline${ }^{59} \mathrm{Co}$ & $0.0565 \pm 0.003$ & $6.4 \%$ & 0.97794 & ${ }^{163} \mathrm{Dy}$ & $0.0106 \pm 0.0016$ & $18.4 \%$ & 0.92426 \\
\hline${ }^{60} \mathrm{Ni}$ & & & & ${ }^{165} \mathrm{Ho}$ & $0.00217 \pm 0.00039$ & $21.3 \%$ & 0.90744 \\
\hline${ }^{63} \mathrm{Cu}$ & $3.57 \pm 0.17$ & $5.8 \%$ & 0.97814 & ${ }^{166} \mathrm{Er}$ & $0.0052 \pm 0.0011$ & $25.3 \%$ & 0.85271 \\
\hline${ }^{66} \mathrm{Zn}$ & $140 \pm 5$ & $4.0 \%$ & 0.99182 & ${ }^{169} \mathrm{Tm}$ & $0.000822 \pm 0.00015$ & $21.9 \%$ & 0.93964 \\
\hline${ }^{71} \mathrm{Ga}$ & $0.0714 \pm 0.0056$ & $9.3 \%$ & 0.9395 & ${ }^{172} \mathrm{Yb}$ & $0.00422 \pm 0.00094$ & $26.6 \%$ & 0.83285 \\
\hline${ }^{72} \mathrm{Ge}$ & $0.00872 \pm 0.00099$ & $13.5 \%$ & 0.92695 & ${ }^{175} \mathrm{Lu}$ & & & \\
\hline${ }^{75} \mathrm{As}$ & & & & ${ }^{178} \mathrm{Hf}$ & $0.00348 \pm 0.00049$ & $16.8 \%$ & 0.90787 \\
\hline${ }^{78} \mathrm{Se}$ & $0.0318 \pm 0.004$ & $15.1 \%$ & 0.93477 & ${ }^{181} \mathrm{Ta}$ & & & \\
\hline${ }^{85} \mathrm{Rb}$ & $0.264 \pm 0.03$ & $13.4 \%$ & 0.93301 & ${ }^{182} \mathrm{~W}$ & & & \\
\hline${ }^{88} \mathrm{Sr}$ & $22.9 \pm 0.6$ & $3.3 \%$ & 0.99794 & ${ }^{197} \mathrm{Au}$ & & & \\
\hline${ }^{90} \mathrm{Zr}$ & $0.119 \pm 0.014$ & $13.8 \%$ & 0.89008 & ${ }^{208} \mathrm{~Pb}$ & $2.12 \pm 0.33$ & $18.4 \%$ & 0.88239 \\
\hline${ }^{93} \mathrm{Nb}$ & $0.0256 \pm 0.0046$ & $21.6 \%$ & 0.82187 & ${ }^{232} \mathrm{Th}$ & $0.0301 \pm 0.0056$ & $22.2 \%$ & 0.80616 \\
\hline${ }^{95} \mathrm{Mo}$ & & & & ${ }^{238} \mathrm{U}$ & $0.0878 \pm 0.0048$ & $6.6 \%$ & 0.97324 \\
\hline
\end{tabular}


Table 12. Elements of 88130 with Repeatability $(<30 \% \mathrm{RSD})$ and Reproducibility $\left(>0.7 \mathrm{R}^{2}\right)$; $\mathrm{N}=8$

\begin{tabular}{|c|c|c|c|c|c|c|c|}
\hline Isotope & Mean $(\mu \mathrm{g} / \mathrm{g})$ & RSD & $\mathrm{R}^{2}$ & Isotope & Mean $(\mu \mathrm{g} / \mathrm{g})$ & RSD & $\mathrm{R}^{2}$ \\
\hline${ }^{9} \mathrm{Be}$ & & & & ${ }^{107} \mathrm{Ag}$ & $0.00331 \pm 0.00052$ & $18.6 \%$ & 0.78672 \\
\hline${ }^{10} \mathrm{~B}$ & $2.85 \pm 0.2$ & $8.3 \%$ & 0.92273 & ${ }^{111} \mathrm{Cd}$ & $0.0383 \pm 0.0021$ & $6.6 \%$ & 0.96591 \\
\hline${ }^{11} \mathrm{~B}$ & $2.87 \pm 0.2$ & $8.3 \%$ & 0.92013 & ${ }^{118} \mathrm{Sn}$ & & & \\
\hline${ }^{23} \mathrm{Na}$ & $95.1 \pm 6.3$ & $7.9 \%$ & 0.92126 & ${ }^{121} \mathrm{Sb}$ & & & \\
\hline${ }^{24} \mathrm{Mg}$ & $111 \pm 6$ & $6.4 \%$ & 0.9495 & ${ }^{125} \mathrm{Te}$ & & & \\
\hline${ }^{27} \mathrm{Al}$ & & & & ${ }^{133} \mathrm{Cs}$ & & & \\
\hline${ }^{31} \mathrm{P}$ & & & & ${ }^{137} \mathrm{Ba}$ & & & \\
\hline${ }^{39} \mathrm{~K}$ & & & & ${ }^{139} \mathrm{La}$ & & & \\
\hline${ }^{43} \mathrm{Ca}$ & $740 \pm 43$ & $7.0 \%$ & 0.94612 & ${ }^{140} \mathrm{Ce}$ & & & \\
\hline${ }^{47} \mathrm{Ti}$ & & & & ${ }^{141} \mathrm{Pr}$ & & & \\
\hline${ }^{51} \mathrm{~V}$ & & & & ${ }^{146} \mathrm{Nd}$ & & & \\
\hline${ }^{52} \mathrm{Cr}$ & & & & ${ }^{147} \mathrm{Sm}$ & & & \\
\hline${ }^{55} \mathrm{Mn}$ & $2.86 \pm 0.2$ & $8.4 \%$ & 0.90353 & ${ }^{153} \mathrm{Eu}$ & & & \\
\hline${ }^{57} \mathrm{Fe}$ & & & & ${ }^{157} \mathrm{Gd}$ & & & \\
\hline${ }^{59} \mathrm{Co}$ & & & & ${ }^{163}$ Dy & & & \\
\hline${ }^{60} \mathrm{Ni}$ & & & & ${ }^{165} \mathrm{Ho}$ & & & \\
\hline${ }^{63} \mathrm{Cu}$ & $0.283 \pm 0.016$ & $6.9 \%$ & 0.95864 & ${ }^{166} \mathrm{Er}$ & & & \\
\hline${ }^{66} \mathrm{Zn}$ & $67.8 \pm 2.1$ & $3.7 \%$ & 0.98812 & ${ }^{169} \mathrm{Tm}$ & & & \\
\hline${ }^{71} \mathrm{Ga}$ & & & & ${ }^{172} \mathrm{Yb}$ & & & \\
\hline${ }^{72} \mathrm{Ge}$ & & & & ${ }^{175} \mathrm{Lu}$ & & & \\
\hline${ }^{75} \mathrm{As}$ & & & & ${ }^{178} \mathrm{Hf}$ & & & \\
\hline${ }^{78} \mathrm{Se}$ & & & & ${ }^{181} \mathrm{Ta}$ & & & \\
\hline${ }^{85} \mathrm{Rb}$ & & & & ${ }^{182} \mathrm{~W}$ & & & \\
\hline${ }^{88} \mathrm{Sr}$ & $4.58 \pm 0.29$ & $7.6 \%$ & 0.93062 & ${ }^{197} \mathrm{Au}$ & & & \\
\hline${ }^{90} \mathrm{Zr}$ & & & & ${ }^{208} \mathrm{~Pb}$ & $1.09 \pm 0.1$ & $10.6 \%$ & 0.926 \\
\hline${ }^{93} \mathrm{Nb}$ & & & & ${ }^{232} \mathrm{Th}$ & & & \\
\hline${ }^{95} \mathrm{Mo}$ & & & & ${ }^{238} \mathrm{U}$ & & & \\
\hline
\end{tabular}


Table 13. Elements of 88210 with Repeatability $(<30 \% \mathrm{RSD})$ and Reproducibility $\left(>0.7 \mathrm{R}^{2}\right)$; $\mathrm{N}=8$

\begin{tabular}{|c|c|c|c|c|c|c|c|}
\hline Isotope & Mean $(\mu \mathrm{g} / \mathrm{g})$ & RSD & $\mathrm{R}^{2}$ & Isotope & Mean $(\mu \mathrm{g} / \mathrm{g})$ & RSD & $\mathrm{R}^{2}$ \\
\hline${ }^{9} \mathrm{Be}$ & & & & ${ }^{107} \mathrm{Ag}$ & & & \\
\hline${ }^{10} \mathrm{~B}$ & $5.68 \pm 1.34$ & $28.3 \%$ & 0.85042 & ${ }^{111} \mathrm{Cd}$ & $0.00984 \pm 0.00148$ & $18.0 \%$ & 0.95858 \\
\hline${ }^{11} \mathrm{~B}$ & $5.73 \pm 1.32$ & $27.6 \%$ & 0.85909 & ${ }^{118} \mathrm{Sn}$ & $0.243 \pm 0.044$ & $21.9 \%$ & 0.93586 \\
\hline${ }^{23} \mathrm{Na}$ & $14600 \pm 700$ & $1.9 \%$ & 0.99835 & ${ }^{121} \mathrm{Sb}$ & & & \\
\hline${ }^{24} \mathrm{Mg}$ & $24.5 \pm 0.9$ & $4.4 \%$ & 0.98358 & ${ }^{125} \mathrm{Te}$ & & & \\
\hline${ }^{27} \mathrm{Al}$ & $645 \pm 62$ & $11.6 \%$ & 0.94698 & ${ }^{133} \mathrm{Cs}$ & & & \\
\hline${ }^{31} \mathrm{P}$ & $27.9 \pm 2.3$ & $10.1 \%$ & 0.98128 & ${ }^{137} \mathrm{Ba}$ & $1.11 \pm 0.06$ & $6.6 \%$ & 0.99453 \\
\hline${ }^{39} \mathrm{~K}$ & $18.9 \pm 3.7$ & $23.3 \%$ & 0.86504 & ${ }^{139} \mathrm{La}$ & & & \\
\hline${ }^{43} \mathrm{Ca}$ & $23.3 \pm 1.8$ & $9.4 \%$ & 0.93437 & ${ }^{140} \mathrm{Ce}$ & & & \\
\hline${ }^{47} \mathrm{Ti}$ & $0.679 \pm 0.152$ & $26.8 \%$ & 0.82079 & ${ }^{141} \operatorname{Pr}$ & $0.00192 \pm 0.00019$ & $12.0 \%$ & 0.94788 \\
\hline${ }^{51} \mathrm{~V}$ & $0.0795 \pm 0.0136$ & $20.5 \%$ & 0.87052 & ${ }^{146} \mathrm{Nd}$ & $0.00577 \pm 0.00053$ & $10.9 \%$ & 0.96001 \\
\hline${ }^{52} \mathrm{Cr}$ & $1.11 \pm 0.17$ & $18.4 \%$ & 0.92338 & ${ }^{147} \mathrm{Sm}$ & $0.0010 \pm 0.00018$ & $21.3 \%$ & 0.90628 \\
\hline${ }^{55} \mathrm{Mn}$ & $1.62 \pm 0.18$ & $13.5 \%$ & 0.94076 & ${ }^{153} \mathrm{Eu}$ & $0.000572 \pm 0.000051$ & $10.7 \%$ & 0.97434 \\
\hline${ }^{57} \mathrm{Fe}$ & $65.9 \pm 8.6$ & $15.6 \%$ & 0.93767 & ${ }^{157} \mathrm{Gd}$ & $0.00114 \pm 0.00012$ & $12.7 \%$ & 0.9706 \\
\hline${ }^{59} \mathrm{Co}$ & & & & ${ }^{163} \mathrm{Dy}$ & $0.000639 \pm 0.000044$ & $8.2 \%$ & 0.98022 \\
\hline${ }^{60} \mathrm{Ni}$ & $0.773 \pm 0.165$ & $25.6 \%$ & 0.83621 & ${ }^{165} \mathrm{Ho}$ & $0.000147 \pm 0.000024$ & $19.8 \%$ & 0.8925 \\
\hline${ }^{63} \mathrm{Cu}$ & $4.89 \pm 0.52$ & $12.8 \%$ & 0.93737 & ${ }^{166} \mathrm{Er}$ & $0.000285 \pm 0.00003$ & $12.5 \%$ & 0.95061 \\
\hline${ }^{66} \mathrm{Zn}$ & $35.4 \pm 2.4$ & $8.1 \%$ & 0.98352 & ${ }^{169} \mathrm{Tm}$ & & & \\
\hline${ }^{71} \mathrm{Ga}$ & $0.0547 \pm 0.0051$ & $11.2 \%$ & 0.94819 & ${ }^{172} \mathrm{Yb}$ & & & \\
\hline${ }^{72} \mathrm{Ge}$ & & & & ${ }^{175} \mathrm{Lu}$ & & & \\
\hline${ }^{75} \mathrm{As}$ & & & & ${ }^{178} \mathrm{Hf}$ & $0.00193 \pm 0.00017$ & $10.8 \%$ & 0.96387 \\
\hline${ }^{78} \mathrm{Se}$ & $0.00788 \pm 0.00266$ & $13.6 \%$ & 0.93673 & ${ }^{181} \mathrm{Ta}$ & & & \\
\hline${ }^{85} \mathrm{Rb}$ & & & & ${ }^{182} \mathrm{~W}$ & & & \\
\hline${ }^{88} \mathrm{Sr}$ & $1.66 \pm 0.06$ & $4.3 \%$ & 0.99306 & ${ }^{197} \mathrm{Au}$ & & & \\
\hline${ }^{90} \mathrm{Zr}$ & $0.0598 \pm 0.0045$ & $9.0 \%$ & 0.99111 & ${ }^{208} \mathrm{~Pb}$ & $0.309 \pm 0.043$ & $16.5 \%$ & 0.92888 \\
\hline${ }^{93} \mathrm{Nb}$ & $0.00317 \pm 0.00045$ & $17.1 \%$ & 0.86572 & ${ }^{232} \mathrm{Th}$ & & & \\
\hline${ }^{95} \mathrm{Mo}$ & $0.0217 \pm 0.0032$ & $17.5 \%$ & 0.91726 & ${ }^{238} \mathrm{U}$ & $0.00143 \pm 0.00033$ & $18.3 \%$ & 0.82572 \\
\hline
\end{tabular}


Table 11 shows that GFX2-83-8002/9 had many elements that were both repeatable and reproducible, so it was fortuitous that sample was made available for use as a duplicate heroin control for sample preparation and anlysis. As one can see from Tables 11, 12 and 13, there are elements that perform better in some heroin samples compared to others. However, between all three heroin samples, almost every element has good repeatability and reproducibility in at least one sample, which presents the argument to measure that element. Initially, it was unclear which elements would lend beneficial information to profiling heroin, therefore many elements were quantified for each sample with the assumption that some may not prove to be useful. However, the alternative of not collecting some useful data was less desirable than having some redundant or useless data.

\subsubsection{Distribution of Elements in Heroin Samples}

The elemental concentrations for the ICP-MS and HR-ICP-MS analysis for all measured isotopes monitored are shown in Figure 22. The logarithmic scale box-andwhisker plots illustrate the range of concentrations for the elements for the heroin samples of each of the four geographic regions. The trends observed were that crustal elements (10$10^{3} \mathrm{mg} / \mathrm{kg}$ ) were present in concentrations approximately two - three orders of magnitude higher than the trace metals $(10 \mu \mathrm{g} / \mathrm{kg}-10 \mathrm{mg} / \mathrm{kg})$, which were found to be one or more orders of magnitude higher than the rare earth elements (REE; $<10^{2} \mathrm{ng} / \mathrm{kg}-10 \mathrm{mg} / \mathrm{kg}$ ). The elements having the highest abundances, with median mass concentrations in the heroin powder greater than $100 \mu \mathrm{g} / \mathrm{g}$, were $\mathrm{Ca}$ and $\mathrm{Na}$. Elements with median mass concentrations in the heroin powder between 1 and $100 \mu \mathrm{g} / \mathrm{g}$ were $\mathrm{Al}, \mathrm{B}, \mathrm{Ba}, \mathrm{Cr}, \mathrm{Fe}, \mathrm{K}$, $\mathrm{Mg}, \mathrm{Mn}, \mathrm{P}, \mathrm{Sr}$ and $\mathrm{Zn}$. The elements with median mass concentrations in the heroin powder 
between 10 and 1000 ng/g were As, Cd, Ce, Co, Cu, Ga, La, Mo, Ni, Pb, Rb, Sb, Sn, Ti, V and $\mathrm{Zr}$. And lastly, the elements with median mass concentrations in the heroin powder below 10 ng/g were Ag, Au, Be, Cs, Dy, Er, Eu, Gd, Hf, Ho, Lu, Nb, Nd, Pr, Se, Sm, Ta, $\mathrm{Te}, \mathrm{Th}, \mathrm{Tl}, \mathrm{Tm}, \mathrm{U}, \mathrm{W}$ and $\mathrm{Yb}$; of these ultra-trace elements only hafnium and uranium were found to be useful in provenance modelling although preference of inclusion was given to zirconium over hafnium due to its higher concentration in the sample (see page 70). The concentrations of rare earth elements were near or below the limits of quantitation with QICP-MS methods for most of the heroin samples.

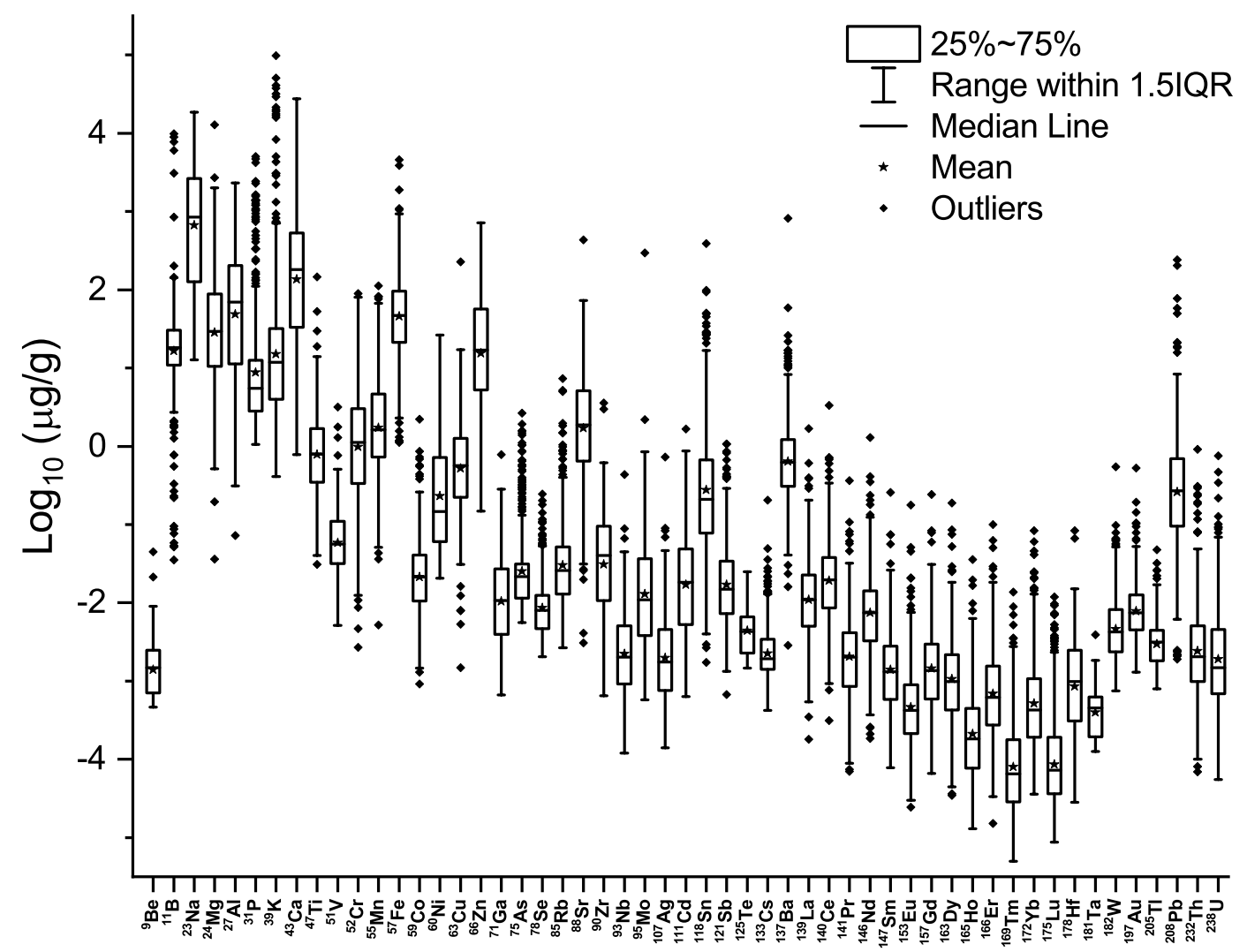

Figure 22. Log-scale box and whisker plots of concentration $(\mathrm{X}, \mu \mathrm{g} / \mathrm{g})$ data obtained on the quadrupole ICPMS. There are three points of data for each sample heroin. Total lead was determined from the sum of the isotopes ${ }^{206} \mathrm{~Pb},{ }^{207} \mathrm{~Pb}$ and ${ }^{208} \mathrm{~Pb}$. 
A logarithmic transformation of the data was necessary to reduce the effect of outlier values on shifting the mean value of the group excessively high. Outliers for some elements had values that were 3 or more orders of magnitude higher than the median value. A logarithmic $\left(\log _{10}\right)$ transformation was made of all data prior to multivariate analysis to avoid loss of data or arbitrary imputation of substitution values for outliers. Loss of data weakens the predictive power of supervised multivariate statistics as well as muddles the data analysis for real samples. Each sample is informative and should not be removed without good cause, such as a gross error in sample preparation or analysis.

To analyze which elements to include in the predictive modelling, the quantiles of each group were analyzed for between-group differences. It was expected that there would be significate overlaps in the distribution of elemental concentration between regions and few, if any, elements would be significantly different between all four regions. For many elements, it was observed that the concentration was only significantly different for one or two of the regions. Figure 23 and Figure 24 demonstrates the distributions of the most useful elements. The logical process of elemental selection was to choose elements that made one of the following distinctions possible: MEX-SA and SA from SEA and SWA, MEX-SA from SA or SEA from SWA. Unfortunately, there was no element in significantly higher abundance in MEX-SA samples as compared to SA samples. 


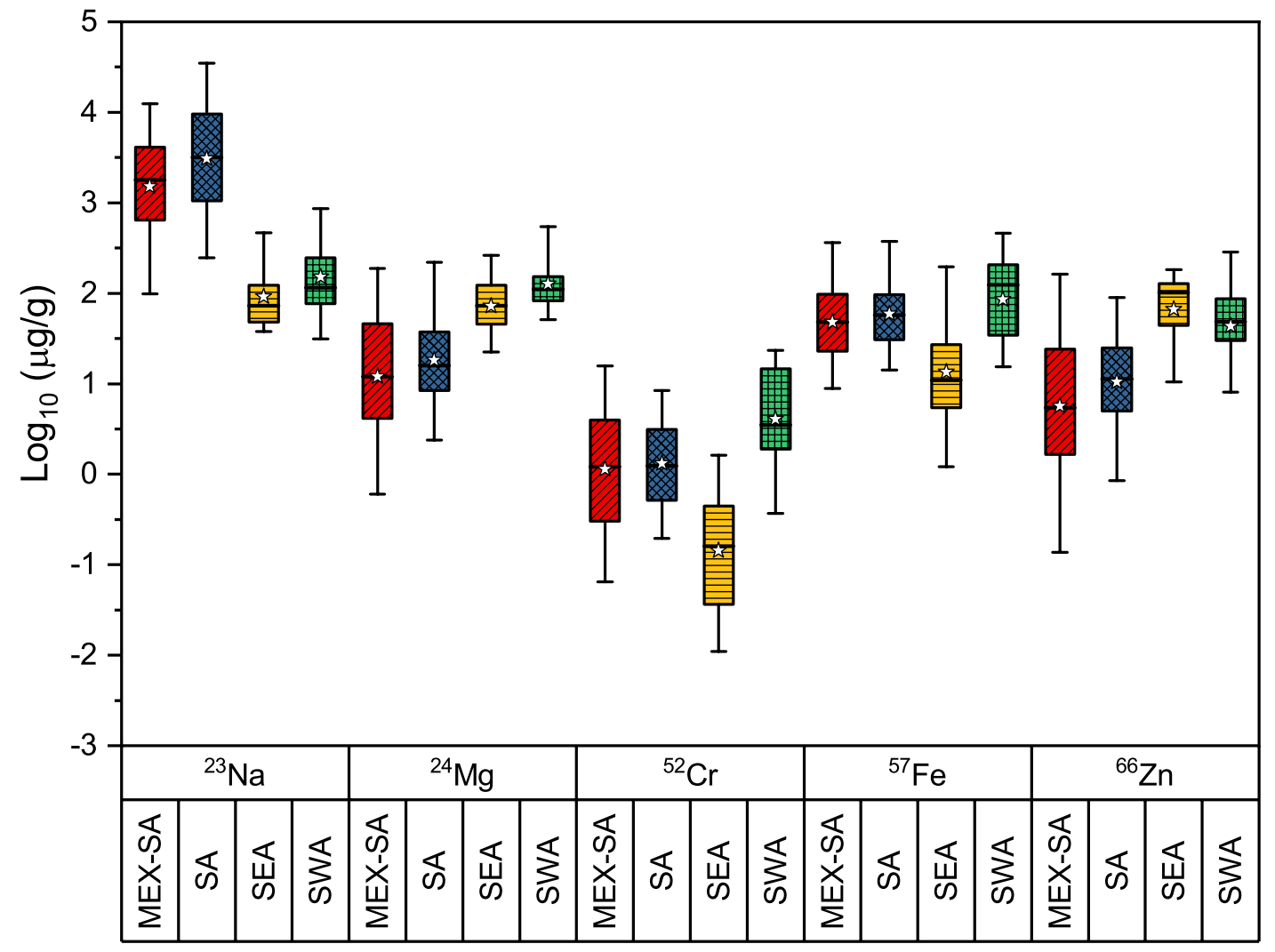

Figure 23. Box plot of $\log _{10}\left({ }^{23} \mathrm{Na},{ }^{24} \mathrm{Mg},{ }^{52} \mathrm{Cr},{ }^{57} \mathrm{Fe}\right.$ and $\left.{ }^{66} \mathrm{Zn}\right) \mu \mathrm{g} / \mathrm{g}$ separated by region. The boxes represent the $25 \%$ to $75 \%$ quantiles and whiskers are the $5 \%$ to $95 \%$ quantiles; the median (line) and mean are also shown (star). MEX-SA is shown in red with diagonal fill, SA is shown in blue with crossed fill, SEA is shown in gold with a horizontal fill and SWA is shown in green with a checkered fill.

Figure 23 shows a grouped box plot of distribution quantiles of $\log _{10}\left({ }^{23} \mathrm{Na},{ }^{24} \mathrm{Mg}\right.$, ${ }^{52} \mathrm{Cr},{ }^{57} \mathrm{Fe}$ and ${ }^{66} \mathrm{Zn}$ ) of the $\mu \mathrm{g} / \mathrm{g}$ elemental concentrations for heroin from each of the four regions. These five elements were chosen because they show the clearest distinction between two or more regions. There is very little overlap in the concentration of ${ }^{23} \mathrm{Na}$, which is vastly higher concentration in MEX-SA and SA than in SEA and SWA samples. The mean concentration of ${ }^{23} \mathrm{Na}$ in MEX-SA and SA samples is greater than $1000 \mathrm{ppm}$, whereas it is approximately $100 \mathrm{ppm}$ in SEA and SA samples. The opposite trend was observed with ${ }^{24} \mathrm{Mg},{ }^{43} \mathrm{Ca}$ and ${ }^{88} \mathrm{Sr}$ where concentrations were generally greater in 
SEA/SWA than in MEX-SA/SA samples. Presumably this could be a result of the regional preferences in a reducing or alkaline agent. Perhaps in the East reduction of morphine to morphine base is performed with $\mathrm{CaOH}$ and in the West, with $\mathrm{NaOH}$. Another possibility is the preference of $\mathrm{CaCO}_{3}$ or $\mathrm{NaHCO}_{3}$ as an alkaline reagent to increase the $\mathrm{pH}$ of the solution after acetylation. However, because the three elements were highly correlated, the only one kept for modelling was ${ }^{24} \mathrm{Mg}$. Both ${ }^{52} \mathrm{Cr}$ and ${ }^{57} \mathrm{Fe}$ tend to show the most distinction between SEA and SWA groups, however the elements are only weakly correlated in the data set. The distribution of ${ }^{66} \mathrm{Zn}$ data overlaps less than $25 \%$ between the MEX-SA/SA and SEA/SWA groups.

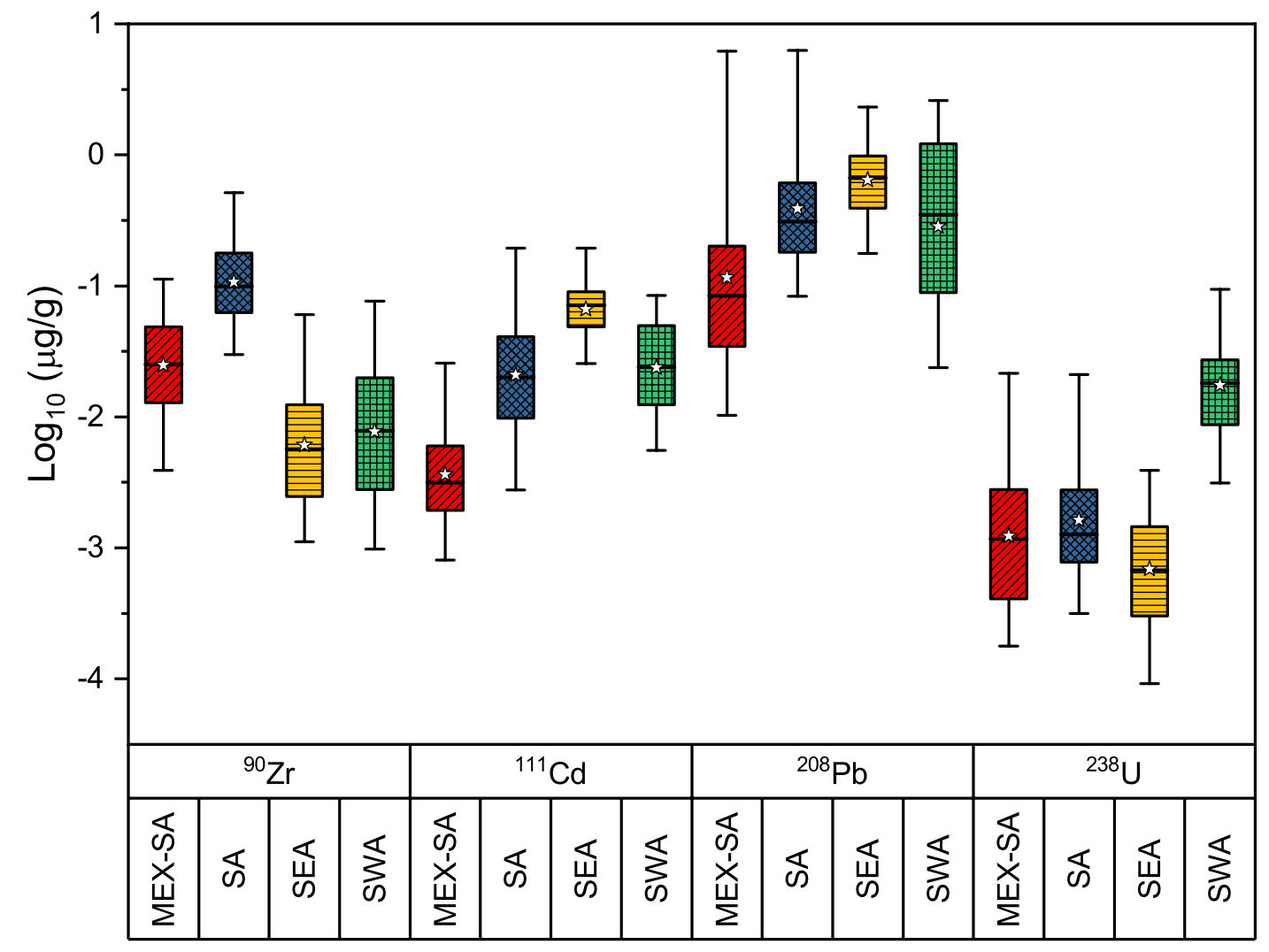

Figure 24. Box plot of $\log _{10}\left({ }^{90} \mathrm{Zr},{ }^{111} \mathrm{Cd},{ }^{208} \mathrm{~Pb}\right.$ and $\left.{ }^{238} \mathrm{U}\right) \mu \mathrm{g} / \mathrm{g}$ concentration by region. The boxes represent the $25 \%$ to $75 \%$ quantiles and whiskers are the $5 \%$ to $95 \%$ quantiles; the median (line) and mean are also shown (star). MEX-SA is shown in red with diagonal fill, SA is shown in blue with crossed fill, SEA is shown in gold with a horizontal fill and SWA is shown in green with a checkered fill. 
The next selection of elements, shown on Figure 24, shows trace elements that have lower concentrations but still are distinguishing between heroin samples from different regions. There are only overlapping values for ${ }^{90} \mathrm{Zr}$ between the upper $25 \%$ and lower $25 \%$ of data for MEX-SA and SA respectively. Additionally, there is almost no overlap in the SA concentration values for ${ }^{90} \mathrm{Zr}$ from either SEA or SWA data. The trend of ${ }^{178} \mathrm{Hf}$ is similar to that of ${ }^{90} \mathrm{Zr}$ as these elements are correlated in both the data set and in nature because of their very similar chemistry and ionic radii, but since they are highly correlated and informative similar ways, ${ }^{178} \mathrm{Hf}$ was removed from the model.

The overlap of MEX-SA and SA data points for ${ }^{111} \mathrm{Cd}$ is even less than for ${ }^{90} \mathrm{Zr}$, and SEA overlaps with less than $25 \%$ of the SA data as well, which also allows some distinction between SEA and SWA with ${ }^{111} \mathrm{Cd}$. There are less than $30 \%$ of MEX-SA and SA samples with overlapping concentrations of ${ }^{208} \mathrm{~Pb}$; it was included because elements differentiating the groups of MEX-SA and SA were very limited. The distinction of SEA from SWA is clear in ${ }^{238} \mathrm{U}$, although there is significant overlap with MEX-SA/SA for SEA with ${ }^{238} \mathrm{U}$. Between the Log-transformed concentration data of the 9 elements, ${ }^{23} \mathrm{Na},{ }^{24} \mathrm{Mg}$, ${ }^{52} \mathrm{Cr},{ }^{57} \mathrm{Fe},{ }^{66} \mathrm{Zn},{ }^{90} \mathrm{Zr},{ }^{111} \mathrm{Cd},{ }^{208} \mathrm{~Pb}$ and ${ }^{238} \mathrm{U}$, a linear discriminant model can be generated to correctly predict the origin of greater than $85 \%$ of blind heroin samples.

\subsubsection{Multivariate Analysis}

Data handling and organization was performed using Excel 2016 (Microsoft, USA). Visual data exploration of data and multivariate analysis was performed using commercial statistical software, JMP 13.0 (SAS, USA), Origin 2017 and 2018 (OriginLabs, USA). Analysis of means, distribution quantiles, and one-way analysis of variance were used to 
rank elements based upon their discrimination power between the 4 heroin producing regions. As mentioned in subchapter 2.4.5, 9 elements were chosen for building the supervised model for heroin profiling and prediction of origin. Three elements were chosen each that explain the differences between MEX-SA and SA, three that explain the differences between MEX-SA/SA and SEA/SWA groups, and three that explain the differences between SEA and SWA.

Multivariate statistical analysis was performed on the data to determine the utility of elemental data obtained via Q-ICP-MS in predicting the geographic origin of unknown data. Linear discriminant analysis was investigated as it is a supervised method of analysis in which multivariate data is reduced into one-dimensional linear equations, consisting of a canonical coefficient and a linear combination of observed data multiplied by a variable coefficient [49].

Equation 3: The form of a linear equation for $\mathrm{n}$ number of variables.

$$
Y=a_{1} X_{1}+a_{2} X_{2}+a_{3} X_{3}+\ldots+a_{n} X_{n}
$$

Linear discriminant analysis (LDA) was used to develop a supervised model for predicting the origin of an unknown heroin sample. DEA-STRL provided approximately $60 \%$ of the samples as a training set for model construction and $40 \%$ of the samples as "blind" samples, with which to test the model. LDA was also performed on the entire data set using leave-one out cross-validation to calculate the number of misclassified samples in a model using all of the samples. As previously explained, the Log-transformed concentration data of 9 elements, ${ }^{23} \mathrm{Na},{ }^{24} \mathrm{Mg},{ }^{52} \mathrm{Cr},{ }^{57} \mathrm{Fe},{ }^{66} \mathrm{Zn},{ }^{90} \mathrm{Zr},{ }^{111} \mathrm{Cd},{ }^{208} \mathrm{~Pb}$ and ${ }^{238} \mathrm{U}$, 
measured on the Q-ICP-MS were used to create a linear discriminant model to correctly predict the origin of the heroin samples.

The results of the LDA prediction were tabulated and are shown on Table 14. The canonical scores of each sample were then plotted using a 3D scatter plot to render the data. The output can be simplified to a classification (confusion) matrix where the known assignments are arranged vertically, and the predicted assignments are listed horizontally. The classification matrix can be generated using leave-one-out (LOO) cross validation in the case where a true set of unknown data is not used. Leave-one-out essentially treats each sample as an unknown and predicts its assignment without participating in the model.

Linear discriminant analysis suffers from over-training of the prediction models when excessive numbers of variables are input in the model. The result of this over-training can result in excellent classification rates for training data sets, but very poor rates of correct classification with blind samples or with truly unknown samples. To avoid overtraining the LDA model, all of the variables that were used were confirmed to have no significant correlation among them. Only a maximum of 9 elements were chosen such that the 9 elements could also be combined with the previously reported ${ }^{87} \mathrm{Sr} /{ }^{86} \mathrm{Sr}$ isotope ratio measurements for a total of 10 elements. The SWA group had smallest sample size of $n=40$ for ${ }^{87} \mathrm{Sr} /{ }^{86} \mathrm{Sr}$ isotope ratio data and it was not wished to exceed a combined number of variables greater than $\mathrm{n} / 4$.

The results shown on Table 14 show clearly that MEX-SA and SA are the most challenging regions to differentiate. Of the "blind" test set about $20 \%$ of the MEX-SA samples and $10 \%$ of SA samples are confused, primarily between the other group. The test 
sample set is correctly associated $97 \%$ and $98 \%$ for SEA and SWA test samples, respectively. Overall the test set is very well associated and differentiated, with correct association over $88 \%$. Differentiation performs well also with $11 \%, 17 \%, 5 \%$ and $11 \%$ of called samples being falsely classified as MEX-SA, SA, SEA and SWA respectively. For SEA the test set is both correctly associated and discriminated $95 \%$ of the time; $98 \%$ of true SEA samples are classified as SEA and only $5 \%$ of samples that are called SEA are not actually SEA.

Table 14. Prediction of Heroin Origin by linear discriminant analysis using $\mathrm{LOG}_{10}$ $\left({ }^{23} \mathrm{Na},{ }^{24} \mathrm{Mg},{ }^{52} \mathrm{Cr},{ }^{57} \mathrm{Fe},{ }^{66} \mathrm{Zn},{ }^{90} \mathrm{Zr},{ }^{111} \mathrm{Cd},{ }^{208} \mathrm{~Pb}\right.$ and $\left.{ }^{238} \mathrm{U}\right) \mu \mathrm{g} / \mathrm{g}$ mass concentrations.

\begin{tabular}{|c|c|c|c|c|c|}
\hline \multirow[b]{2}{*}{ Actual Origin } & \multicolumn{5}{|c|}{ Predicted Count for Training Set } \\
\hline & MEX-SA & SA & SEA & SWA & $\%$ Correct \\
\hline MEX-SA $(n=168)$ & 142 & 23 & 0 & 3 & $85 \%$ \\
\hline SA $(n=293)$ & 40 & 249 & 0 & 4 & $85 \%$ \\
\hline SEA $(n=116)$ & 1 & 3 & 107 & 5 & $92 \%$ \\
\hline SWA $(n=97)$ & 0 & 0 & 0 & 97 & $100 \%$ \\
\hline SUM $(n=674)$ & 183 & 275 & 107 & 109 & $88 \%$ \\
\hline$\%$ Confusion & $22 \%$ & $9 \%$ & $0 \%$ & $11 \%$ & \\
\hline & \multicolumn{5}{|c|}{ Predicted Count for Test Set } \\
\hline Actual Origin & MEX-SA & SA & SEA & SWA & $\%$ Correct \\
\hline MEX-SA $(n=192)$ & 151 & 35 & 1 & 5 & $79 \%$ \\
\hline $\mathrm{SA}(\mathrm{n}=195)$ & 19 & 173 & 3 & 0 & $89 \%$ \\
\hline SEA $(n=96)$ & 0 & 1 & 93 & 2 & $97 \%$ \\
\hline SWA $(n=57)$ & 0 & 0 & 1 & 56 & $98 \%$ \\
\hline $\operatorname{SUM}(n=540)$ & 170 & 209 & 98 & 63 & $88 \%$ \\
\hline$\%$ Confusion & $11 \%$ & $17 \%$ & $5 \%$ & $11 \%$ & \\
\hline & \multicolumn{5}{|c|}{ Predicted Count for Combined Data } \\
\hline Actual Origin & MEX-SA & SA & SEA & SWA & $\%$ Correct \\
\hline MEX-SA $(n=360)$ & 310 & 41 & 4 & 5 & $86 \%$ \\
\hline $\mathrm{SA}(\mathrm{n}=488)$ & 65 & 416 & 3 & 4 & $85 \%$ \\
\hline SEA $(n=212)$ & 1 & 6 & 194 & 11 & $92 \%$ \\
\hline SWA $(n=154)$ & 0 & 0 & 1 & 153 & $99 \%$ \\
\hline $\operatorname{SUM}(\mathrm{n}=1214)$ & 376 & 463 & 202 & 173 & $88 \%$ \\
\hline$\%$ Confusion & $18 \%$ & $10 \%$ & $4 \%$ & $12 \%$ & \\
\hline
\end{tabular}


Additionally, the overall performance is equitable in the both training and test set, overall at $88 \%$ correct association, which further strengthens the argument that the model is not over-trained. In the situation of an over-trained LDA, the modelling of the training set would be noticeably superior in performance compared to the fitting of the test set. The intention of this profiling method would be to strengthen its efficacy by adding authentic data points to the model as time goes on, or completely rebuilding the model in the event that the nature of heroin production happens to change in one or more regions.

The LDA model can be rebuilt in a matter of seconds with a statistical analysis software equipped with discriminant functions, such as JMP or Origin, which were used in this research. New elemental data can be added to (or removed from) the discriminant function if available. Once variables are selected, the discrete, categorical data of the authentic samples' origins are used for grouping. A canonical function is generated and reported, usually in 3 dimensions, each a linear function consisting of a weighed coefficient for all variables in the model, as described in Equation 3 on page 72. The output of the LDA function includes the coefficients of the linear functions for each dimension (canonical 1, 2 and 3) as shown on Table 15. The canonical functions are constructed in such a way that each dimension serves to separate data orthogonally.

Multiplied by the canonical coefficients, each element's concentration value (Log transformed) is linearly summed to locate the date within 1,2 or in this case, 3-dimensional space. The prediction of each sample's origin is made depending on the shortest vector from the closest group mean to the data point of the sample. 
Table 15. Canonical coefficients of linear discriminant analysis by $\mathrm{LOG}_{10}\left({ }^{23} \mathrm{Na},{ }^{24} \mathrm{Mg}\right.$, $\left.{ }^{52} \mathrm{Cr},{ }^{57} \mathrm{Fe},{ }^{66} \mathrm{Zn},{ }^{90} \mathrm{Zr},{ }^{111} \mathrm{Cd},{ }^{208} \mathrm{~Pb},{ }^{238} \mathrm{U}\right)$.

\begin{tabular}{r|rrrr}
\hline \hline Variable & Canon1 & Canon2 & Canon3 \\
${ }^{23} \mathrm{Na}$ & 0.8016 & 0.0875 & 0.1195 \\
${ }^{24} \mathrm{Mg}$ & -0.5085 & -0.0510 & 0.3350 \\
${ }^{52} \mathrm{Cr}$ & 0.2785 & -0.5115 & 0.5035 \\
${ }^{57} \mathrm{Fe}$ & 0.2868 & -0.3416 & 0.4077 \\
${ }^{66} \mathrm{Zn}$ & -0.5482 & 0.1450 & 0.2570 \\
${ }^{90} \mathrm{Zr}$ & 0.7251 & 0.2866 & 0.4497 \\
${ }^{111} \mathrm{Cd}$ & -0.4804 & 0.5373 & 0.6219 \\
${ }^{208} \mathrm{~Pb}$ & -0.1998 & 0.3571 & 0.3527 \\
${ }^{238} \mathrm{U}$ & -0.1163 & -0.5671 & 0.6942
\end{tabular}

\subsection{Conclusion}

The performance of the discriminant model for provenance determination method for elemental impurities in heroin prepared by microwave-assisted acid digestion analyzed by ICP-MS had excellent performance in being able to correctly classify the region of origin in authentic heroin samples. Overall, $88 \%$ of the test sample set of authentic heroin was correctly classified to the region of origin as previously determined by the HSP at DEA-STRL. For the test sample set, SEA and SWA were correctly assigned for over 95\% of the samples. Moreover, other regions were only confused $5 \%$ of the time with SEA, meaning if a sample was called SEA, it was actually a SEA sample $95 \%$ of the time. For the other three groups the confusion rates were between $11-17 \%$.

The excellent association rates of SEA and SWA heroin were as a result of very clear differences observed in the concentrations of $\mathrm{Cr}, \mathrm{Fe}, \mathrm{Cd}$ and $\mathrm{U}$ between SEA and SWA samples. In addition, comparison of elemental concentrations of $\mathrm{Na}, \mathrm{Mg}, \mathrm{Zn}$ and $\mathrm{Zr}$ 
made it possible to distinguish samples originating from the Western hemisphere (MEXSA and SA) from samples originating from the Eastern Hemisphere (SEA and SWA).

There remains room for improvement in the classification of heroin samples of MEX-SA and SA origins. Only four elements were found to differentiate MEX-SA from SA samples, $\mathrm{Zr}$, $\mathrm{Cd}$, $\mathrm{Hf}$ and $\mathrm{Pb}$. Because $\mathrm{Zr}$ and $\mathrm{Hf}$ are so chemically similar, and well correlated in the samples, Hf was not included in the model. Furthermore, SA samples were higher in concentration for all of these elements. There was not a single element for which MEX-SA samples had consistently higher concentrations than SA samples. It was not surprising that most of the confusion of determining provenance was between the MEXSA and SA samples.

The results suggest that the rare earth elements are not in sufficient abundance to be useful by analysis by ICP-MS unless the method is optimized for more sensitive detection of these elements and pushing the background signal lower, perhaps by in-house distillation of acids and water. It was an unfortunate decision in the design of the experiment to use the elements of $\mathrm{Li}, \mathrm{Sc}, \mathrm{Y}, \mathrm{In}, \mathrm{Tb}$, and $\mathrm{Bi}$ as internal standards for the digestion as they could have provided valuable information. In particular, scandium and yttrium, which are often correlated with lanthanide-series rare earth elements in plants and soils [50], could have been measured instead of rare earth elements, resulting in a simplified elemental menu.

Based upon the results and observations, improvements can be made in future efforts to profile heroin by its elemental impurities. For example, the elements shown herein to have utility for provenance determination could be specifically targeted rather 
than attempting to quantify so many other, inessential (with respect to profiling heroin) elements of the periodic table. Using a reduced element menu would simplify analysis as well as facilitate the use of customized external calibration solutions. Calibration solutions with varying concentration could be prepared from single element standards to provide better results than a commercially purchased mixture. For example, an element with high abundance, like sodium, can be calibrated over a range of $\mathrm{mg} / \mathrm{L}$ concentrations, while a trace element, like uranium, calibrated over a range of $\mathrm{ng} / \mathrm{L}$ concentrations.

Important aspects of the validation of this method and for the entire research effort were the inclusion of SRM (NIST 1570a), a control heroin sample, and instrumental QC solutions which were purchased from a separate vendor from the calibration stock mixture. The inclusion of the SRM allowed for the extraction efficiency of the digestion as well as the analysis to be monitored from day to day. The control heroin sample allowed inter-day repeatability to be assessed on elements recovered from an actual heroin sample. The QC solution important to check for accuracy in the calibration stock mixture as prepared by vendor and alert to any error in preparation of calibration solutions. Samples should also be analyzed as soon after digestion as possible and results should be rigorously monitored for consistency in performance for all elements of interest. If possible, samples should be prepared and analyzed from multiple sources to avoid inter-day biasing, which can alter statistical analysis. 


\section{CHAPTER 3. FORENSIC SAMPLE COMPARISON OF HEROIN}

\subsection{Abstract}

Forensic sample comparison of illegal drug evidence can be used to associate samples of drug taken at different points of seizure. In this work is reported a demonstration of a statistical means of performing pair-wise comparisons of heroin samples on the basis of their elemental compositions. A profile of 11 isotopes, ${ }^{23} \mathrm{Na},{ }^{24} \mathrm{Mg},{ }^{27} \mathrm{Al},{ }^{51} \mathrm{~V},{ }^{52} \mathrm{Cr},{ }^{55} \mathrm{Mn}$, ${ }^{66} \mathrm{Zn},{ }^{88} \mathrm{Sr},{ }^{90} \mathrm{Zr},{ }^{111} \mathrm{Cd}$ and ${ }^{137} \mathrm{Ba}$ was determined to be most useful in correctly associating as well as discriminating between heroin samples in forensic pairwise comparisons. Samples were prepared by microwave-assisted acid digestion and quantitatively analyzed by inductively coupled plasma-mass spectrometry to measure the elemental abundance within each sample.

With a match criterion of 3 standard deviations for quantitative data of 11 elements, the rate of discrimination and association were $99.7 \%$ and $95.8 \%$, respectively. Type 1 error rates (false exclusion) were found to be $4.2 \%$ and type 2 error (false inclusion) rates were $0.03 \%$. Furthermore, a set of unknown samples suspected of having commonality were compared to test the method performance with simulated casework samples. Several of the unknown samples were found to be indistinguishable. This is the first known work to report a method for performing pair-wise forensic sample comparison of heroin by targeting elemental impurities. It is also the first to use authentic heroin samples to develop and test the method and report error rates using a large test set. The availability of a method in which samples can be prepared, analyzed and compared in less than 24 hours with no 
necessary chemical derivatizations nor separations is expected to be of great use to forensic drug chemists.

\subsection{Introduction}

Forensic comparisons based upon the analysis of the minor and trace elements of evidence samples are useful for investigation and intelligence purposes to link evidence found at an accident or crime scene to evidence recovered elsewhere. For instance, glass evidence recovered from the clothing of a hit and run victim can be elementally analyzed and compared to evidence recovered from a suspect's vehicle to determine if the suspect was the perpetrator in the crime [51-53]. Soils recovered at a crime scene and/or from physical evidence can be compared by their elemental composition $[54,55]$. In cases such as improvised explosives, copper wires have been shown to be able to be associated based upon their trace elemental composition [56]. Trace element analysis and analysis of radiogenic lead isotope ratios have been reported for comparing bullets and bullet fragments in forensic evidence comparison cases [57].

Forensic drug analysis is typically conducted with the objective of identifying and quantifying the illegal compounds within seized materials that are suspected of being illegal drugs by law enforcement officers. However, sample comparison of illegal drug evidence can help investigators to solve an individual criminal case as well as support the fight against illegal drug trafficking through gathering intel on distribution networks [40, $41,58]$. The majority of the works on forensic heroin comparisons have been performed by analysis of associated organic components [5, 6, 40, 59-61]. Taking samples from the same container is an overly simplistic representation of a forensic comparison scenario and 
is not an accurate reflection of how the method might perform for samples that are unknown; however, it is necessary given that one must use samples that are known to share the same intra-sample chemical composition. Incidentally, the manuscript by Klemenc reports the same rate of false inclusion (1/24) that was observed in this dissertation [61]. There have been attempts to perform source comparisons using trace elements [40-42], however these have reported observations of sample grouping and similarity rather than clearly describing a method that could be used to compare two samples in forensic casework.

Chan et al. reported the first demonstration of trace elements being used for streetlevel sample investigation, however accurate false inclusion and false exclusion rates could not be provided as the samples were not authenticated by other means [42]. The following year, another manuscript by Chan et al. took the investigation further with a larger samples set, while performing unsupervised multivariate analysis, PCA, to observe the inter-sample grouping on the basis of the elements being measured [41]. However, neither of the manuscripts reported on a method to perform a pair-wise forensic sample comparison of two or more heroin samples on the basis of their elemental compositions.

Inductively coupled plasma-mass spectrometry (ICP-MS) was chosen as the method of inorganic analysis of heroin samples. The benefits of ICP-MS are its wide dynamic range and its ability to quantitatively measure many different elements. Statistical comparisons were made on the basis of the resultant quantitative analytical data of inorganic impurities within each heroin sample. The presence of elemental impurities is 
expected to be, in major part, resultant from chemical adulteration and contamination during processing, packaging and handling.

Chemical analysis can make sample comparisons at the micro level between a heroin dealer's supply and the drug user. For example, comparison of drug samples can help law enforcement to determine if a sample recovered from a local heroin dealer is chemically indistinguishable from that recovered from an overdose victim. The penalties for distribution of drugs could be harsher with the evidence that drugs have resulted in fatalities. With the increased prevalence of synthetic opioids being mixed with heroin, there may be increased culpability by a dealer who knowingly increases the overdose potential of their product. A method to make chemical comparisons is especially helpful for local law enforcement working such cases.

Chemical analysis can be used to match heroin samples at the macro level of bulk shipments and traffickers. Forensic sample comparison can also allow investigators to determine if samples taken from seizures in different cities or at ports of entry are indistinguishable, and therefore possibly part of related trafficking organizations. For example, samples seized in Dubai could be found indistinguishable from samples seized from a clandestine lab in Afghanistan and traced back to a terrorist organization using heroin to finance its operation. The technique of elemental comparison will be another point of comparison to organic compound analysis which attempts to compare the opiate adulterant profiles within a particular sample.

The hypothesis for forensic sample matching based upon the elemental similarity of two samples is that samples of the same source and production run will be able to be 
associated on the basis of their elemental compositions. But it is not at all expected that a sample's elemental profiles will remain unchanged from the sample's illicit production to consumption by the end user. It is known, especially for heroin, that sample can be largely cut with other organic substances. Each adulterant will contribute elemental impurities to the sample, as shown in 5.3 the contribution may be quite significant. However, samples that are processed, handled, packaged, adulterated and repackaged in common are expected to contain an indistinguishable profile of minor and trace elements.

In highly refined bulk samples, the concentration of elemental impurities is expected to be lower relative to the adulterated, street samples. Therefore, the elemental fingerprinting technique will be most useful for two forensic case scenarios wherein samples have changed possession and not subsequently been adulterated. Transactions where upon adulteration is less likely to occur are primarily at the trafficking level during large-quantity, bulk exchanges and secondarily on the small-scale during dealer to user exchanges. The assumption is that the majority of the adulterants are added to the sample before the drugs change hands on the medium to small scale in order to increase the mass of the sample and seller's profit during down-stream transactions.

During a study conducted to determine the geographic origin of heroin samples based upon their elemental composition [62], it was noted that several of the samples provided by the US Drug Enforcement Administration's Special Testing and Research Laboratory (DEA-STRL) may be useful to evaluate the technique's usefulness in sample to sample comparisons as well. For the profiling research over 400 heroin samples were available. While there was limited background information available on the samples, it was 
noted during the course of sample inventory that several separate containers of heroin seemed to have a serialized labeling convention. For example, there were some samples labeled as XXXXY-A, XXXXY-B or XXXXZ001, XXXXZ002, etc. and had, from a visual assessment, very similar heroin in each package. The observed similarity of the heroin and the labeling previously mentioned, led to the assumption that these samples might be duplicates or perhaps recovered from a common seizure. The samples that were suspected of being chemically related were reserved as test samples for the comparison method.

For the purpose of testing the sample comparison method in simulated casework, all of the samples were compared to one another without any assumption of grouping. Sample A was of Mexican origin (MEX), samples B-F were Southeast Asian (SEA), and samples G-K were Southwest Asian (SWA). Samples marked B, C, and D also shared a common origin being from Southeast Asia (SEA) and were labeled as XXXX7, XXXX8, and XXXX9, respectively. The similar numbering suggested that there may be some additional similarity amongst these sample types.

There were no samples available from South America (SA) that met this requirement. The over-representation of SWA and SEA in the test set was not seen as a limitation because of the practical application of a sample-to-sample comparison method for illegal drugs. Most likely, a country such as in the United States, has one or two main supply lines of heroin. Since 2000, the main sources of heroin in the US are Mexico and South America [29]. Therefore, associating (or differentiating) heroin samples from one or 
two regional types will be the most likely scenario for a forensic lab attempting sample-tosample comparisons.

Interpretation of evidence and reported findings in forensic analysis has become reliant upon statistical analysis of results in order to accurately represent the significance of findings, rather than relying upon the subjective interpretation of the expert. In the case of forensic sample comparisons, a widely accepted means of presenting the results are in the form of likelihood ratios from Bayesian statistics [58]. The results are presented as a ratio between the probabilities of the analyst's observation given two competing hypotheses. Determination of the elemental profile uniqueness for each sample was beyond the scope of this work, which precludes the calculation of likelihood ratios. However, the error rates for the entire method were determined and used in a similar fashion. For example, if an analyst makes an observation that two drug evidence samples are indistinguishable, the results are reported as a random match probability, which is defined as the probability of making the match given the hypothesis that the samples are, in fact, chemically indistinguishable divided by the probability of a match given the hypothesis that the match was made purely by random chance.

To calculate random match probability, the probability of observing false positives was determined from a set of 120 heroin samples known to be chemically distinct from one another. Elemental data from 120 samples, 30 from each of the four regions, Mexico, South America, Southeast Asia and Southwest Asia, were compared to assess the rate of discrimination (5400 total comparisons) and type 2 error rates (false inclusions). The 30 samples from each region were compared to the 90 other samples not of that region for a 
total of 5400 total pairwise comparisons $(120 \times 90 \div 2=5400)$. The probability of observing false negatives was determined from a set of 24 samples ( 3 replicates) prepared and analyzed in duplicate on separate days, for a total of 24 pairwise comparisons

\subsection{Methods and Materials}

\subsubsection{Standard Mixtures}

The internal element standard mixture (ICP-MS 71D), the calibration element standard mixtures (ICP-MS 71A, 71B and 71D), and additional elemental standards (Au and Rh) were purchased from Inorganic Ventures, USA. The internal standard mixture, ICP-MS 71D, was prepared in $0.8 \mathrm{M} \mathrm{HNO}_{3}$ at a concentration of $200 \mathrm{ppb}{ }^{6} \mathrm{Li}, \mathrm{Sc}, \mathrm{Y}$, In, $\mathrm{Tb}$, and $\mathrm{Bi}$, in order to account for the dilutions pre- and post-digestion, which produced a final concentration of approximately $1 \mathrm{ppb}$ in the samples for analysis. The spiked internal standard was used to verify complete digestion and monitor for losses during samples transfer post-digestion.

Calibration solutions consisted of ICP-MS mixes 71A, 71B, 71D and Au elemental standard. The elements within the mixes were: ICP-MS Mix 71A (Ag, Al, As, B, Ba, Be, Ca, Cd, Ce, Co, Cr(III), Cs, Cu, Dy, Er, Eu, Fe, Ga, Gd, Ho, K, La, Lu, Mg, Mn, Na, Nd, Ni, P, Pb, Pr, Rb, S, Se, Sm, Sr, Th, Tl, Tm, U, V, Yb, and Zn), 71B (Ge, Hf, Mo, Nb, Sb, Si, Sn, Ta, Te, Ti, W, and Zr), 71D (Bi, In, ${ }^{6} \mathrm{Li}, \mathrm{Sc}, \mathrm{Tb}$, and Y). The calibration solution was prepared as a stock solution in $0.8 \mathrm{M} \mathrm{HNO}_{3}$ at a concentration of $300 \mathrm{ng} / \mathrm{g}$. The external calibration samples were prepared by serial dilution from $300300 \mathrm{ng} / \mathrm{g}$ using an Ovation ${ }^{\circledR}$ Macro10 electronic pipette (Vistalab Technologies, USA) with a serial dilution function to $100,30,10,3.0,1.0,0.3,0.1,0.03$ and $0 \mathrm{ng} / \mathrm{g}$. 
Rhodium elemental standard diluted to $100 \mathrm{ppb}$ in $0.8 \mathrm{M} \mathrm{HNO}_{3}$ was supplied externally into the nebulizer of the ICP-MS, a 7700x (Agilent Technologies, USA) for monitoring plasma inconsistencies and instrumental variation over the course of the analytical run. It was determined that $\mathrm{Rh}$ had not been present at any appreciable concentration in the initial batch of 50 heroin samples and was therefore a suitable candidate element for the purpose of an instrumental check standard.

All reagent solutions were prepared using DI $\mathrm{H}_{2} \mathrm{O}$ from a Milli-Q water system (MilliporeSigma, USA) with a boron filter pack and Optima-grade nitric acid (Fisher Scientific, USA).

\subsubsection{Digestion Method}

Heroin samples were dissolved by microwave-assisted acid digestion using a Milestone Ethos UP digestion oven (Milestone Scientific, Italy) with Milestone SK-15, polyether ether ketone (PEEK) digestion vessels. Each vessel was outfitted with additional accessories that allowed the insertion of 3 quartz vials with PEEK caps, referred to as micro-insert vials. The quartz micro-inserts facilitated digestion of three heroin sample replicates within a single, larger vessel. The addition of the smaller volume, quartz microinserts also permitted the digestion of smaller mass heroin samples with lower volumes of expensive, Optima-grade nitric acid.

For each digestion replicate, heroin samples of mass 30 to $100 \mathrm{mg}$ were weighed directly into the quartz micro-inserts on a Mettler AE 240 (Mettler-Toledo, USA) balance to $\pm 0.1 \mathrm{mg}$. In order to pre-wet the heroin sample before digestion, $0.2 \mathrm{~mL}$ of the internal standard mixture was added to each quartz micro-insert with an Ovation ${ }^{\circledR}$ Macro10 
electronic pipette (Vistalab Technologies, USA) including reagent blanks, SRM and duplicate control heroin samples. The pipette tips were pre-rinsed in freshly prepared 0.8 $\mathrm{M} \mathrm{HNO}_{3}$ prior to all applications. Lastly, $2.3 \mathrm{~mL}$ of Optima-grade nitric acid (Fisher Scientific, USA) was added to each micro-insert.

In order to both reduce the formation of nitrous and nitric oxide gas during sample oxidation and uniformly regulate the temperature of each of the three micro-inserts, $10 \mathrm{~mL}$ of an equal volume mixture of DI $\mathrm{H}_{2} \mathrm{O}$ and ACS-grade hydrogen peroxide (Fisher Scientific, USA) was added to the inner PEEK sleeve of the SK-15 digestion vessel. The micro-inserts were lowered into the $\mathrm{H}_{2} \mathrm{O}_{2}$ bath, but the bath solution never came into direct contact with the samples. Careful assembly of the SK-15 digestion vessel was done following the instruction from the manufacturer using safety-spring caps, which allowed for venting and resealing of the vessel in the event of excessive pressurization during the digestion. Pressures exceeding 75 bars could be experienced within the digestion vessel and incorrect assembly could result in sample loss or contamination, damage to the equipment or physical injury to the operator. The microwave digestion oven follows the oven program given on Table 3 on page 34 .

\subsubsection{Instrumental Analysis Method}

The instrumental parameters for the Agilent 7700x quadrupole ICP-MS can be found on Table 4 and Table 5. The instrument was equipped with an ASX-510 autosampler (Teledyne Cetac, USA). 


\subsection{Data analysis}

There was no removal of outlier measurements or other pretreatment of data, save from background subtraction using the calculated concentration of the method blanks and multiplication of the elemental concentration in solution $(\mu \mathrm{g} / \mathrm{L})$ by the sample's dilution factor to produce a mass concentration in the heroin sample itself $(\mu \mathrm{g} / \mathrm{kg})$.

Once the analytical results were transformed to mass concentrations for each element, it was necessary to determine how best to compare two samples. Two match criteria were evaluated. For the first match criterion, a comparison interval was created for each element; the interval was defined as the average $\pm n \times s$, where $s$ is the standard deviation and $n$ is a coefficient of $s$. If the comparison interval for one sample (the "known") overlapped with the comparison interval for another sample (the "questioned") for all 11 elements, the two samples were considered to be indistinguishable. For the second match criterion, the average of the questioned sample was compared to the comparison interval (average $\pm n \times s$ ) of the known sample; thus, this approach does not take the standard deviation of the questioned sample into account. If the average of the questioned sample fell within the known comparison interval for all 11 elements, the two samples were considered to be indistinguishable. The second match criterion has been reported for the comparison of forensic glass evidence and is prescribed in the glass standard ASTM E2927 [63]. Unlike the first match criterion described, the second approach is asymmetrical (i.e., the conclusion depends on which sample is used to calculate the comparison interval). Thus, every pair was compared twice so that each sample was treated as the known. For each match criterion, a minimum standard deviation $(s)$ equal to 
$3 \%$ of the average was enforced; thus, the comparison interval is more accurately defined as the average $\pm n \times s$ or the average $\pm n \times 0.03 \times$ average, whichever is greatest. Establishing a minimum standard deviation reduces the risk of false exclusions [63].

Table 16. Figures of merit for analysis of the spinach control by Q-ICP-MS ( $\mathrm{n}=62$ ). Missing recovery values indicate elements not reported on the certificate of analysis of NIST SRM 1570a and an asterisk $(*)$ indicates elements that were reported only as information values.

\begin{tabular}{rrrcc}
\hline \hline \multirow{2}{*}{ Analyte } & LOD $(\mu \mathrm{g} / \mathrm{g})$ & \multicolumn{1}{c}{ MEAN } & Inter-day RSD $(\%)$ & RECOVERY $(\%)$ \\
\hline${ }^{23} \mathrm{Na}$ & 4.2 & $19300 \pm 600$ & 10 & 105.8 \\
${ }^{24} \mathrm{Mg}$ & 0.032 & $9370 \pm 250$ & 10 & $104.1^{*}$ \\
${ }^{27} \mathrm{Al}$ & 0.18 & $214 \pm 8$ & 14 & 68.9 \\
${ }^{51} \mathrm{~V}$ & 0.002 & $0.57 \pm 0.02$ & 14 & 100.2 \\
${ }^{52} \mathrm{Cr}$ & 0.004 & $1.6 \pm 0.06$ & 15 & 108.2 \\
${ }^{55} \mathrm{Mn}$ & 0.003 & $82 \pm 2$ & 10 & 99.9 \\
${ }^{66} \mathrm{Zn}$ & 0.086 & $82 \pm 1.9$ & 9 & 107.2 \\
${ }^{88} \mathrm{Sr}$ & 0.002 & $60 \pm 1.6$ & 11 & \\
${ }^{90} \mathrm{Zr}$ & 0.002 & $0.13 \pm 0.006$ & 19 & 98.9 \\
${ }^{111} \mathrm{Cd}$ & 0.003 & $2.8 \pm 0.07$ & 10 & \\
${ }^{137} \mathrm{Ba}$ & 0.006 & $6.8 \pm 0.19$ & 11 &
\end{tabular}

In order to evaluate the type 1 error rate (false exclusion), the match criteria described above were applied to the 24 samples analyzed in duplicate. If one (or more) element(s) was found to be distinguishable for a pair of samples, that pair was considered a false exclusion. In order to evaluate the type 2 error rate (false inclusion), each of the 30 samples for one region was compared to all samples from a different region, resulting in 5400 comparison pairs $[30 \times(90+60+30)]$. Note that the number of comparison pairs is doubled $(10,800)$ for the second match criterion since, as described above, this match criterion is asymmetrical. If all 11 elements overlapped for a pair of samples, the pair was considered a false inclusion. By comparing two samples from different regions, the possibility of encountering matches within the same region was avoided. Coefficient values 
(n) for multiplying the standard deviations were evaluated between 1.0 and 4.0 , at intervals of 0.5 . The false exclusion and inclusion rate for both match criteria and for all coefficients tested are shown in Figure 25.

The selection of useful elements for forensic comparison was also performed during the procedure for determining match criteria. The selected elements $\left({ }^{23} \mathrm{Na},{ }^{24} \mathrm{Mg},{ }^{27} \mathrm{Al},{ }^{51} \mathrm{~V}\right.$, ${ }^{52} \mathrm{Cr},{ }^{55} \mathrm{Mn},{ }^{66} \mathrm{Zn},{ }^{88} \mathrm{Sr},{ }^{90} \mathrm{Zr},{ }^{111} \mathrm{Cd}$, and ${ }^{137} \mathrm{Ba}$ ) were chosen on the basis of their analytical performance with the SRM NIST 1570a shown on Table 16, and for producing the lower rates of false inclusions and exclusions than were observed when using other elements. All pairwise comparisons were calculated using the R programming language (RStudio version 1.0.143) [64]. To reduce computing time, parallelization was used via the packages "parallel," "doParallel," and "foreach" [64-66]. After the R script finishes all pairwise comparisons, the results are stored in an excel file (using package "openxlsx") [67].

\subsection{Results}

\subsubsection{Calculating Error Rates and Random Match Probability}

As mentioned previously, two types of match criteria were evaluated. From Figure 25 , it is clear that the first approach (labeled "s $\mathrm{s}_{1}$ to $\mathrm{s}_{2}$ ") was more suitable for the heroin sample matrix than the second approach (labeled " $\mathrm{x}_{1}$ to $\mathrm{s}_{2}$ "). When the questioned average was compared to the known interval, intra-sample variation had significant effects on the results ( $>30 \%$ of duplicate samples were falsely excluded). The high false exclusion rate was the result of one aberrant replicate in the questioned sample, significantly affecting the questioned average, and ultimately leading to the average falling outside the known interval. With a more homogeneous sample matrix, the intra-sample variation is minor and 
the average of a small number of questioned replicates is more likely to fall within the known interval. However, heroin samples are not produced in a controlled environment and are subject to contamination by minerals and unnatural materials that may alter the elemental composition greatly from one replicate to the next.

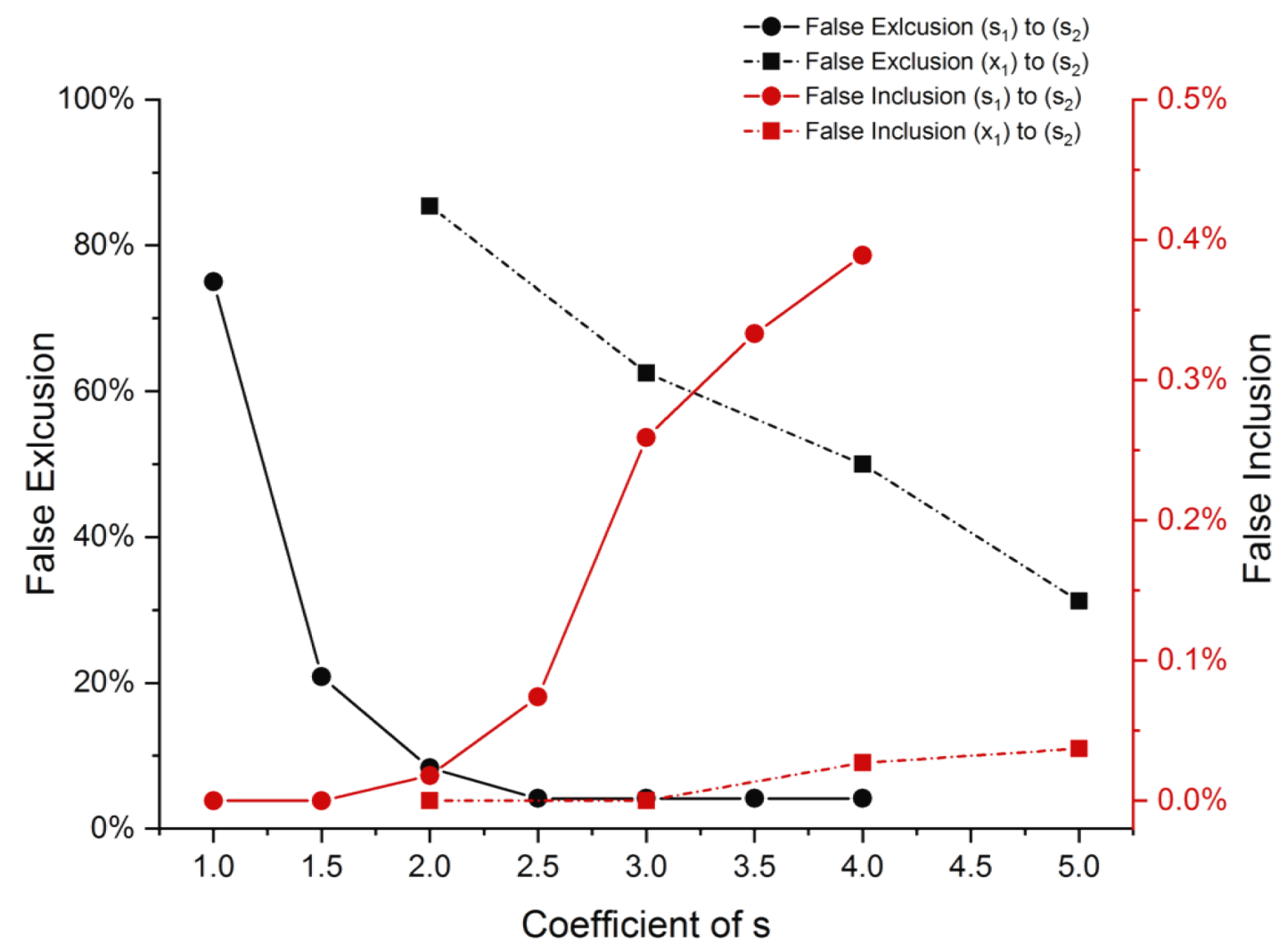

Figure 25. Plot of type 1 and type 2 error rates with increasing coefficient of $s$ used for match criteria. The error rates using match criteria comparing the range of uncertainty of the test set ( $\left.\mathrm{s}_{1}\right)$ to that of the reference set $\left(\mathrm{s}_{2}\right)$ are shown in black. The error rates using match criteria comparing the mean value of the test set $\left(\mathrm{x}_{1}\right)$ to the uncertainty of the reference set $\left(\mathrm{s}_{2}\right)$ are shown in red.

The results of the data analysis showed that a coefficient of 3.0 multiplied by the samples' standard deviation produced the optimal balance between the type 1 and type 2 error rates ( $4.2 \%$ and $0.3 \%$, respectively). A plot of the effect of the coefficient on the error rates is shown on Figure 25. Although a coefficient of 2.5 produced a lower type 2 error 
rate and the same type 1 error rate compared to a coefficient of 3.0, it is suspected that the estimation of the type 1 error rate is limited by the small sample size ( 24 samples). For standard deviation coefficients between 2.5 and 4.0 there was only a single false exclusion (1/24), producing the same type 1 error rate across that range (Table 17). A larger interval $( \pm 3 s)$ was selected to minimize the risk of a false exclusion; this is especially important for actual casework samples, which are expected to exhibit greater inter-sample variation than duplicate samples taken from the same container, as was done in this study. Furthermore, an interval of $\pm 3 s$ contains $>99 \%$ of normally distributed data, giving additional significance to the choice of $\pm 3 s$.

Based upon the determination of the error rates, a random match probability can be calculated for a match of all 11 elements. The significance of a random match probability calculation is that an expert witness can compare the probability of an observation of evidence with respect to the hypothesis posed by the prosecution $\left(\mathrm{H}_{0}\right)$ and the hypothesis posed by the defense $\left(\mathrm{H}_{1}\right)$. A random match probability of 1 means that there is an equal support of the hypotheses that a forensic comparison match occurred through random chance or through veritable similarity in their elemental compositions. For matches of all 11 elements, the calculated random match probability is 386 , calculated by $1 \div$ the type 2 error rate. The numerator is 1 because the chance of observing a match at all 11 elements, given the hypothesis that the samples are of common source is $100 \%$. The denominator is the probability of observing a match of all 11 elements given random chance, which is the rate of type 2 errors. A way of phrasing the significance of the random match probability is to say, it is 386 times more likely that the elemental profiles of the heroin samples match as a result of having a common source rather than being matched by random chance. 
Matching fewer than 11 elements were said to have distinguished the two samples due to their being of different sources, being of different processing batches, or because the elemental profile had been altered by means of contamination.

Table 17. Summary of error rates and calculation of random match probability over a range of match criteria. $\mathrm{ND}=$ not defined

\begin{tabular}{|c|c|c|c|c|c|}
\hline $\begin{array}{l}\text { coefficient } \\
\text { of } \mathrm{s}\end{array}$ & $\begin{array}{l}(\mathrm{n}=24) \\
\text { false } \\
\text { exclusions }\end{array}$ & $\begin{array}{l}\text { false } \\
\text { exclusion } \\
\text { rate }\end{array}$ & $\begin{array}{l}(\mathrm{n}=5400) \\
\text { false } \\
\text { inclusion }\end{array}$ & $\begin{array}{l}\text { false } \\
\text { inclusion } \\
\text { rate }\end{array}$ & $\begin{array}{l}\text { Random } \\
\text { Match } \\
\text { Probability }\end{array}$ \\
\hline 4 & $1 / 24$ & $4.17 \%$ & $21 / 5400$ & $0.39 \%$ & 257 \\
\hline 3.5 & $1 / 24$ & $4.17 \%$ & $18 / 5400$ & $0.33 \%$ & 300 \\
\hline 3 & $1 / 24$ & $4.17 \%$ & $14 / 5400$ & $0.26 \%$ & 386 \\
\hline 2.5 & $1 / 24$ & $4.17 \%$ & $4 / 5400$ & $0.07 \%$ & 1350 \\
\hline 2 & $2 / 24$ & $8.33 \%$ & $1 / 5400$ & $0.02 \%$ & 5400 \\
\hline 1.5 & $4 / 24$ & $20.83 \%$ & $0 / 5400$ & $0.00 \%$ & ND \\
\hline 1 & $10 / 24$ & $75.00 \%$ & $0 / 5400$ & $0.00 \%$ & ND \\
\hline
\end{tabular}

With respect to the samples with were falsely included at a $\pm 3 s$ range interval for match, in each of the 14 pairs of mismatched samples, at least one member was one of four "bad-apple" samples. The cause of the mismatching of the "bad-apple" samples were their elements are either in low abundance, which is common for many other samples, and they overlap from the 3\% RSD minimum $s$ value or there was a moderate to high concentration of element(s) present, but the sampling (or less likely, the analytical) variation was large enough to have $\pm 3 s$ range overlap with samples with lower (or higher) abundances of the element(s). 


\subsubsection{Matching Simulated Case Samples}

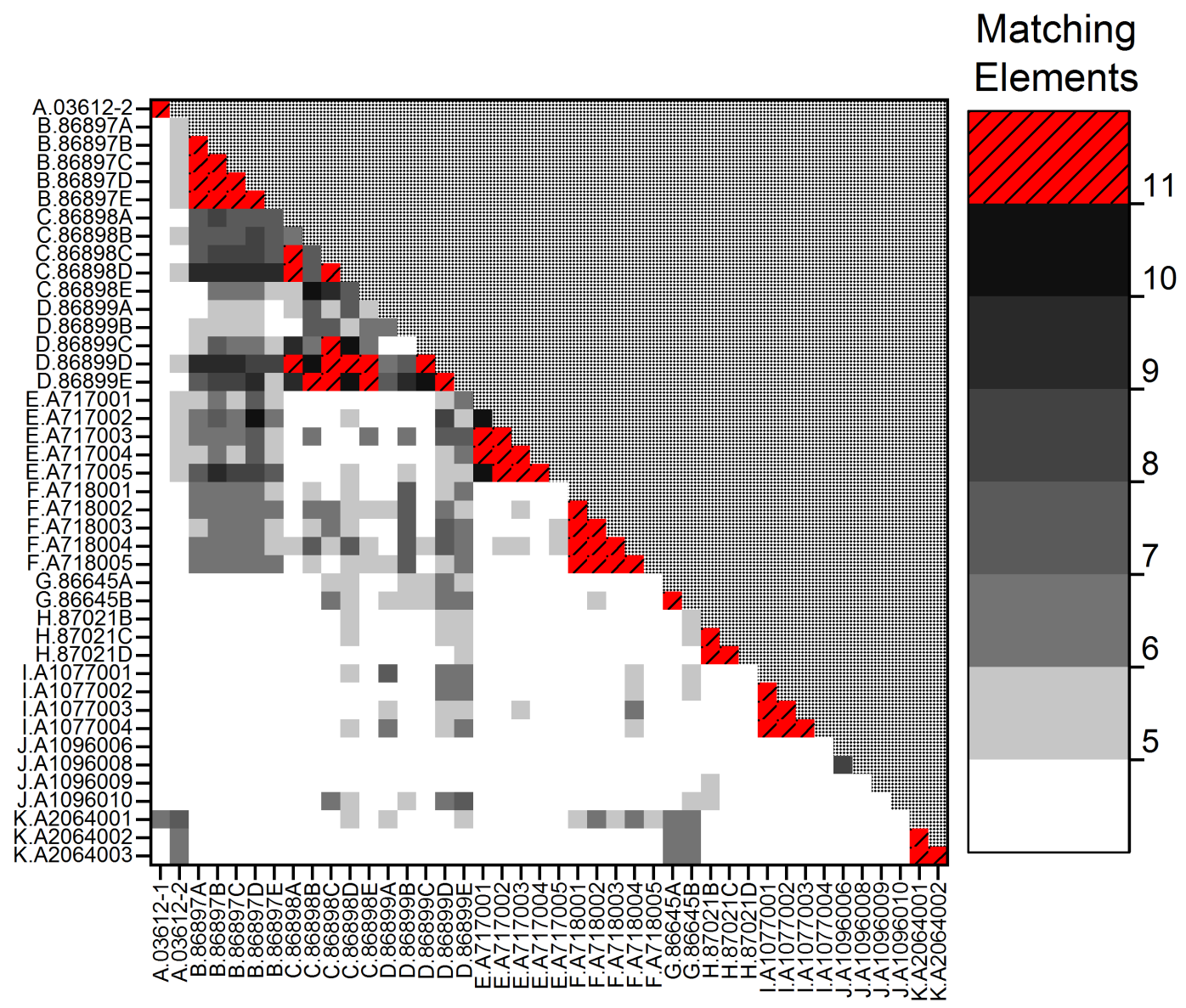

Figure 26. Heatmap showing samples with $\leq 5$ matching elements in white and 6-10 matching elements in increasingly darker shades of gray. The samples matching at all 11 elements are shown in red with striped fill and generally are found along the diagonal, suggesting similar origin with other members of their test groups. Samples were not compared to themselves.

For demonstration of the method on simulated casework samples, two approaches were undertaken. The first was simply to compare each sample to all of the others in order to identify trends among the samples with serial labels and between samples with similar numbering and/or region of origin. As seen in Figure 26, nearly all sample pairs that matched at all 11 elements were part of the same group, suspected from their label. However, not all of the groups showed similarity (e.g., group J). 
There were several matched elements in groups B, C, D, and E, which were all Southeast Asian samples. Since samples B, C, and D were labeled sequentially, interesting challenges were expected with the samples. It should be noted that the commonality of the samples is unknown apart from their region of origin. Samples B, C, and D were SEA specimens that were seized in Australia and provided to DEA for characterization by their signature methods, but it is unknown whether these samples were part of the same seizure or what level of interrelatedness, if any, should be expected.

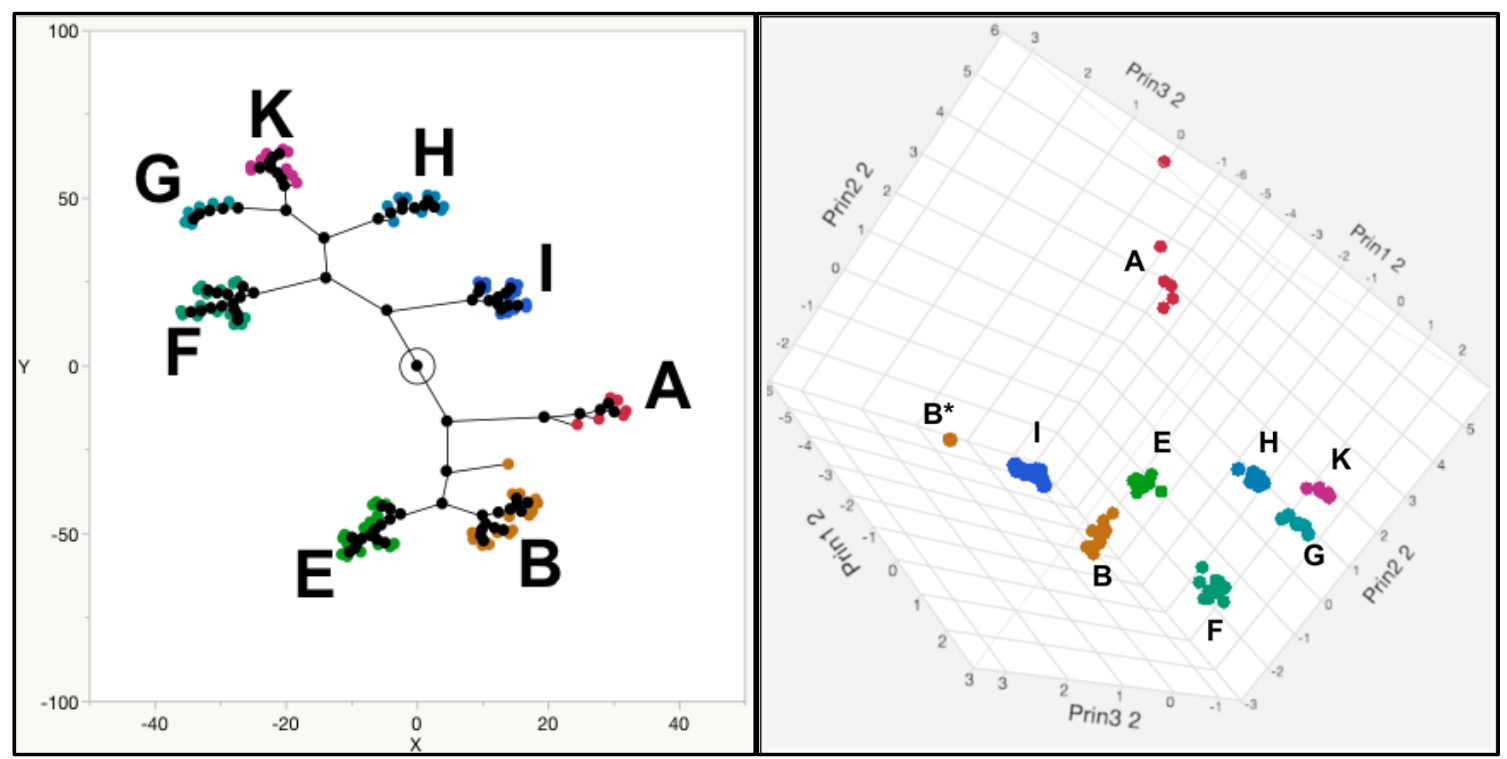

Figure 27. Hierarchical cluster analysis constellation plot (left) and principle component analysis plot (right) of sample replicates of groups A, B, E, F, G, H, I and K.

It is clear that samples within groups A, B, E, F, G, H, I and K are all very closely related as nearly all samples within each group matched at all 11 elements. It is very likely that they are from the same source and perhaps even the same processing batches. The relatedness of all replicates of groups A, B, E, F, G, H, I and K are represented visually with unsupervised multivariate analysis plots on Figure 27 using JMP 13 (SAS, USA). One 
replicate of group B is observed to be deviant from the group, which demonstrates the effects of intra-sample variation.

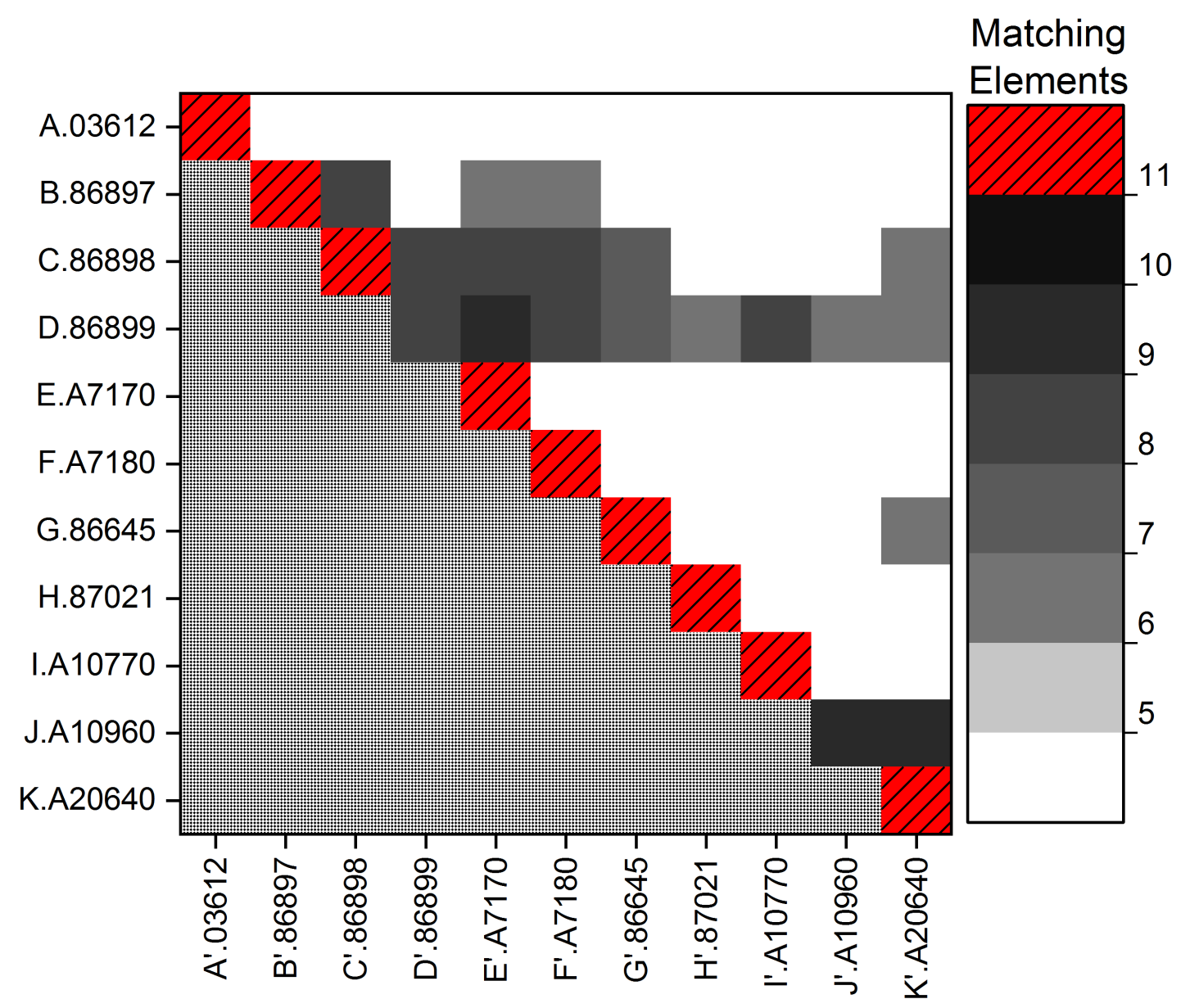

Figure 28. Heatmap of test samples (A'-K') compared to the aggregate profiles of a reference group of samples (A-K). Samples with $<5$ matching elements are shown in white and 5-10 matching elements in increasingly darker shades of gray. The samples matching at all 11 elements are shown in red with stripe fill.

The second approach to simulate forensic casework was to batch all of the samples of each letter group (A-K) into a reference group and remove one sample as a test sample $\left(A^{\prime}-K^{\prime}\right)$. The selected test samples were prepared and analyzed in separate runs from all reference samples within each group. Reference samples ranged in numbers from one to five samples, each with three replicates for a total of 3-15 replicates in a particular reference 
group. The purpose of this exercise was to simulate a case where a bulk seizure of heroin was made, and a single seized evidence sample was compared to the profile of the bulk. The results of this analysis are shown in Figure 28 and suggest that a single sample can be matched to a pool of heroin samples. However, it is unknown whether these samples are actually part of common seizures.

It is vitally important that the samples of the seizure first be compared internally (as was done in Figure 26) in order to confirm that the samples of the seizure are indistinguishable from each other. It is quite possible that samples in a seizure may not be part of the same processing run and possibly not even from a common source. Grouping samples without first comparing them increases the chance of a false inclusion, since the standard deviation interval will be expanded by using non-matching samples. All of the test samples matched at all 11 elements to their respective groups except D' and J', which was not surprising as these samples did not seem to have much interrelatedness among them (Figure 26). A total of 10 elements matched between test sample J' and its reference group, when there were actually very few matches made between the individual samples in the J group. It is clear that by grouping the unrelated samples based upon assumptions of similarity, the probability of exhibiting a match increased.

\subsection{Conclusion}

The method reported in this paper is the first description of a simple approach for the analysis and interpretation of evidence involving pairwise comparisons of heroin samples based upon their minor and trace elemental profiles. However, recognizing the limitation in the interpretation of the results is crucial. For example, as was observed, very 
closely related samples have higher probability of being matched as opposed to completely unrelated samples. Therefore, if the local heroin market is flooded with a single source of heroin, it may be challenging to distinguish uncut sample batches from one another, although that observation could be useful information from the perspective of gathering intelligence about the local heroin supply. Fortunately, from the standpoint of chemical discrimination, middle- and lower-tiered dealers are likely to dilute their product with one (or more) adulterant compound(s) before selling it to a heroin user, which will impart unique elemental features to the final product and will actually support efforts to associate and discriminate samples using the described method.

The best practice would be to compare evidence samples taken from multiple available evidentiary exhibits, as well as vary the sampling point within each exhibit to avoid bias due to sampling. It is advisable that reference samples be taken from multiple containers as well as from multiple sampling locations (such as exterior and interior of sample bulk) in order to account for sample variation. Careful documentation must be maintained especially in the case of bulk seizures such that evidence is properly labeled to allow for comparison of all samples to one another. Batching of samples from separate packages should be avoided completely, as the assumption that the samples are of a common production batch may be incorrect, even if they are part of the same bulk seizure. Comparing samples will reveal if they are, in fact, indistinguishable and post-hoc pooling can be done afterward. It is also recommended that when using the match criterion described for sample-to-sample comparisons, $>3$ sample replicates should be collected, sample mass permitting, to account for the intra-sample variation. 
The ability to assign a statistical quality to the match criterion allows forensic experts to testify without overstating the importance of a match. In the case where samples are recovered from a suspect dealer and a user, if the two samples are found to be indistinguishable using the match criterion described, it does not necessarily follow that the suspect dealer supplied the user. Using a match criterion of $\pm 3.0 \mathrm{~s}$ a match of all 11 elements described in this report will produce a random match probability of 384 . However, increasing the sample size of the type 1 error analysis may result in a lower type 1 error rate and consequently a larger value for the random match probability. Using a match criterion of $\pm 2.5 \mathrm{~s}$ is stricter and produces a random match probability of 1,428 . As such, an analyst may opt to report the random match probability of the lowest, however using more stringent match criterion may increase the chances of making false exclusions, type 1 errors, when applied to actual casework samples. 


\section{CHAPTER 4. STRONTIUM ISOTOPE RATIO ANALYSIS OF HEROIN}

\subsection{Introduction}

Forensic drug analysts around the globe continue to gather strategic or tactical intelligence information on heroin trafficking by conducting profiling studies of opium and processing-related impurities found in heroin [7, 36, 37, 39, 41, 42]. The presence of adulterants and/or diluents is also being used for tactical comparisons by investigating agencies. The U.S. Drug Enforcement Administration's (DEA) Special Testing and Research Laboratory (STRL) is entering its $40^{\text {th }}$ year of Heroin Signature Program (HSP) analyses and currently employs ultra-high performance liquid chromatography [34], gas chromatography mass spectrometry (GC-MS) with liquid-liquid extraction and subsequent derivatization [15, 33], Static headspace GC-MS [68], and isotope ratio mass spectrometry $[20,21]$ as signature methodologies. The presence, absence, and relative abundances of opium and other manufacturing impurities including residual organic solvents are determined quantitatively or semi-quantitatively using these analytical techniques. The results are compared to the databases of authentic heroin samples to assign geographic origin classifications such as Southeast Asia (SEA), Southwest Asia (SWA), South America (SA) or SA-like heroin manufactured in Mexico (MEX-SA).

Heroin is clandestinely manufactured from opium via morphine using a series of chemical processing steps. Depending on the efficacy of isolation and purification steps employed and the knowledge and proficiency of the clandestine processors, the final heroin product can vary from a crude brown to a very highly refined white powder. Or the product can advertently be manufactured crudely to supply to certain markets (i.e., black tar heroin 
shipments). The basic morphine isolation chemistry and the subsequent synthetic reaction scheme to convert morphine into heroin are consistently similar amongst many regions; however, the "regional recipes" remain unique in their own ways by using different apparatus, chemicals, and organic solvents.

One of the challenges the HSP has been encountering is the dynamic nature of heroin production. For example, opium poppy cultivation could use alternative varieties of poppy seeds and, as a result, the established opium alkaloid profile for that particular region can be altered. The opium alkaloid profiles from all poppy-growing regions constitute the necessary foundation for many HSP methods and any changes in them may require modifications for HSP. In addition, one specific region can adopt another region's heroin manufacturing recipes, thus narrowing the differences between the chemical signatures of the final products. Foreseeing such potential deviations in heroin production, the DEA laboratory has explored new research areas and has engaged in new analytical and geochemical method development.

Collaboration with the U.S. Geological Survey and National GeospatialIntelligence Agency has led to the preliminary analysis of more than 100 heroin and opium samples to develop a profiling method on the basis of trace elemental data (DEA laboratory unpublished research). The results of this research suggested that the variation in elemental composition between samples of different regional origins may not be significant compared to the variation between samples originating from the same region. Other research has been undertaken to characterize the elemental composition of heroin and the results have shown promise for sample matching [7, 36, 39, 41]. Unfortunately, many studies attempting to 
profile heroin based upon element composition have produced inconclusive findings regarding the utility of elemental concentrations for provenance determination. This may have been because of a limited sample size or incomplete representation from one or more geographic regions.

Analyses of stable isotope ratios have been made for heroin samples and shown to have potential for sample comparison $[31,69]$ as well as for origin prediction $[19,21,32$, 70]. There have also been endeavors to determine provenance for geographic heroin origin by means of stable isotope ratio analysis. Through analysis of stable isotope ratios $\delta^{15} \mathrm{~N}$ and $\delta^{13} \mathrm{C}$, it has been shown to be possible to differentiate between all four regional groups following deacetylation of heroin to the morphine starting material [19]. The analysis of the stable isotope ratios of elements $\mathrm{H}, \mathrm{C}, \mathrm{N}$ and $\mathrm{O}$ is affected by the presence of cutting agents and other organic impurities, as well as the semi-synthetic acetyl groups of heroin. Researchers in this field would agree that mass diluents as well as synthetic and other semisynthetic compounds present in street level heroin would have complicating effects on the analysis, which would require chemical modification and separation to correct for the bias to the measurement of these light element stable isotope ratios.

Unfortunately, there are no data available for elemental concentrations in either the parent poppies (Papaver somniferum) or the opium latex, compared to those in the heroin product produced from that material. Thus, it is unknown whether the origin of the elements found in the heroin is biologically relevant or, instead, is present from external contamination (including potential contaminants from the reagents, solvents, and vessels used in the extraction and subsequent conversion of morphine into heroin). Therefore, it 
was desirable to target the ${ }^{87} \mathrm{Sr} /{ }^{86} \mathrm{Sr}$ systematic as an analyte that may discriminate between heroin samples based upon regional characteristics rather than process-related variation.

The radiogenic strontium isotope composition of illicit heroin may be the result of contribution from natural and anthropogenic sources. One possibility is the geogenic transfer of the bedrock composition as recorded in weathered top-soil to the $P$. somniferum plant and then to the plant extract and carried through to the heroin final product. While the authors are unaware of any measurements of radiogenic Sr isotopes in P. somniferum biomass, substantial evidence has been mounting on unfractionated transference of the soil strontium isotope signature to various plants and plant products [71-73]. Specifically, Song et al [74] showed that the ${ }^{87} \mathrm{Sr} /{ }^{86} \mathrm{Sr}$ isotopic signature of soil types associated with basaltic, granitic and carbonate bedrocks is preserved with fidelity within the plant biomass that grows atop the soil.

It is therefore reasonable to expect that $P$. somniferum also maintains the geochemical composition of the soil that can potentially pass onto processed products such as heroin without fractionation, considering the large masses of $\mathrm{Sr}$ isotopes. While this opens the possibility of associating a link between illicit heroin and the growing grounds of $P$. somniferum, its successful application hinges on prior knowledge of the bedrock/soil Sr isotope composition. A first step in this direction is mapping the geographic expanses of growing grounds, followed by analysis of local soil composition. Considering the inherent limitations in accessing some of these regions, a more feasible approach maybe to compare the existing geological maps of exposure rock formations that identify ranges of Sr isotope composition and developing a model for the representative $\mathrm{Sr}$ isotope signatures 
for these regions. Similar studies have been previously conducted with success in North America [75-77].

It is important to also recognize other potential mechanisms that can exert control on the $\mathrm{Sr}$ isotope composition of heroin. For example, Degryse and coauthors [78] found that the $\mathrm{Sr}$ isotope composition of groundwater, which may not be representative of the bedrock, can leave a significant imprint on the bulk composition of the plant biomass. Because of the large masses of $\mathrm{Sr}$ isotopes, the biological influence of the $P$. somniferum plant on fractionating Sr isotopes during plant uptake and metabolic processes is unlikely, however it also needs further investigation.

The potential anthropogenic contributions of strontium should be not ignored and may be sourced from cutting material used to dilute heroin after production, packaging/handling contamination, and/or contamination from equipment or chemicals used in the process of heroin production. Constraining the contribution from such contaminants is the most challenging aspect of this new toolbox. Nevertheless, while the individual components of local operations are difficult if not impossible to tease out geochemically, the average influence of natural and anthropogenic sources of $\mathrm{Sr}$ isotope composition on large regional scales can be discerned with great fidelity as we demonstrated in this study, making this a potentially powerful new tool in geochemical fingerprinting of heroin. Preliminary exploration into the isotopic systematics of $\mathrm{B}$ and $\mathrm{Pb}$ was also done, but analysis of $\mathrm{B}$ and $\mathrm{Pb}$ isotope ratios was found to be challenging by this means of sample preparation. 
To the best of the authors' knowledge this manuscript reports the first analysis of radiogenic ${ }^{87} \mathrm{Sr} /{ }^{86} \mathrm{Sr}$ isotope ratios of authentic seized heroin samples for the exploratory purpose of differentiating heroin based upon its geographic and/or processing origins. It also is the first to report ${ }^{87} \mathrm{Sr} /{ }^{86} \mathrm{Sr}$ isotope ratio values for the NIST SRM 1570a (trace elements in spinach leaves), sometimes referred to as IAEA-331 in the literature, which can be used for inter-laboratory comparison in geochemical fingerprinting of plants and illicit drugs.

\subsection{Methods and Materials}

\subsubsection{Samples and Standards}

One hundred and eighty-six authentic (known country of origin), unadulterated and undiluted illicit heroin samples that were made available by the DEA STRL were analyzed for their radiogenic strontium isotope ratios. Samples were categorized as having originated from Mexico manufactured using a South American recipe (MEX-SA, n=44), South America (SA, n=61), Southwest Asia (SWA, n=40) or Southeast Asia (SEA, n=41).

These authentic samples were clandestinely produced heroin samples seized either in the country of production or in direct transit to the United States from a source country of production. Upon seizure and opening of the original packaging, a sub-sample was collected and stored in glass vials for analysis. The exact origin locations (e.g., villages or towns) of these samples were typically unknown. It is also worth noting that different types of metallic and plastic utensils are commonly used for heroin production. Mixing and/or contamination after production are also expected with the packaging and shipping. The objective of this research was to profile heroin shipments that are trafficked to the United 
States without any bias or additional purification steps, and any elemental contamination from cooking pots to packaging has to be considered unavoidable and part of the sample matrix.

Calibration standards for inductively coupled plasma-mass spectrometry (ICP-MS) were prepared from stock solutions of 71A (Inorganic Ventures, USA). Internal standard solutions were also obtained from Inorganic Ventures. Calibration verification standards (CCV) were prepared from single element solutions (Ricca, USA). All were prepared in $0.6 \mathrm{M} \mathrm{HNO}_{3}$ using $\mathrm{MQ}$ water (EMD Millipore, Germany) at $>18 \mathrm{M} \Omega$ and Optima grade nitric acid (Fisher Scientific, USA). The digestion recovery standard was NIST SRM 1570a (Trace Elements in Spinach Leaves; NIST, USA). The Sr isotope standard was SRM 987 (Strontium Carbonate Isotopic Standard); NIST, USA). One of the heroin samples (SWA) was provided with sufficient mass quantity to be used as a daily heroin control standard. The ${ }^{87} \mathrm{Sr} /{ }^{86} \mathrm{Sr}$ values of this heroin control sample were included with the SWA sample set.

\subsubsection{Microwave-assisted acid digestion}

Approximately $0.03-0.1 \mathrm{~g}$ of each sample was weighed to $\pm 0.0001 \mathrm{~g}$ on a Mettler AE240 (Mettler Toledo, USA) balance into a $4 \mathrm{~mL}$ quartz digestion vessel using a disposable polypropylene (PPE) spatula to transfer the samples from the glass sample containers. Polyetherketone (PEK) caps were only removed when absolutely necessary to minimize exposure to the open-air environment.

All sample manipulation was conducted inside class-100 fume hoods. An electronic pipette (Ovation Macro10; Vistalabs, USA) was used to deliver $2.5 \mathrm{~mL}$ of concentrated Optima $\mathrm{HNO}_{3}$ (Fisher Scientific, USA) to each digestion vessel containing the sample, 
rinsing residual powder down from the walls of the vials. Three quartz vessels, each containing a separate replicate mass of the same heroin sample were placed into a perfluoroalkoxy alkane (PFA) digestion bomb that contained a buffer solution of $10 \mathrm{~mL}$ $20 \% \mathrm{v} / \mathrm{v} \mathrm{H}_{2} \mathrm{O}_{2}$ (ACS grade; Fisher Scientific, USA). The vessels were assembled and loaded into a microwave oven (ETHOS-UP; Milestone Inc, USA) with the thermal probe position inserted within a reference vessel as described in EPA method 3052 [45].

The digestion program consisted of a 15-minute ramp from room temperature to $220{ }^{\circ} \mathrm{C}$. The temperature was held for 15 minutes and then passively cooled to room temperature within 1 hour of the completion of the program. The digested volume, which was typically reduced to approximately $1.5 \mathrm{~mL}$, was quantitatively transferred to $15 \mathrm{~mL}$ centrifuge tubes with $>18 \mathrm{M} \Omega \mathrm{MQ}$ water (EMD Millipore, Germany) to approximately 13 $\mathrm{mL}$ of total volume. The final digestion volume was determined to the nearest $0.01 \mathrm{~g}$ on a Sartorius LC4200 (Sartorius AG, Germany) balance after subtracting the mass of the centrifuge tube. Each digestion run included a blank, NIST SRM 1570a, and the heroin control sample.

The average concentration of nitric acid in 6 unique digestion samples was found to be $1.87 \pm 0.31 \mathrm{M} \mathrm{HNO}_{3}$. For the $\mathrm{Sr}$ ratio measurements, $2 \mathrm{~mL}$ aliquots of the digestion samples were used without further dilution for extraction chromatography and $\mathrm{Sr}$ isotope analysis. For the ICP-MS measurements, aliquots of the digestion samples were further diluted to approximately $0.6 \mathrm{M} \mathrm{HNO}_{3}$ with $\mathrm{MQ}$ water.

All digestion vessels were cleaned in a blank microwave run using $3 \mathrm{~mL}$ trace metal grade nitric acid (Fisher Scientific, USA) and soaked in $6 \mathrm{M} \mathrm{HNO}_{3}$ overnight at $90^{\circ} \mathrm{C}$ in a 
covered PFA beaker. All PFA beakers and polypropylene spatulas were soaked in $6 \mathrm{M}$ $\mathrm{HNO}_{3}$ overnight followed by immersion in a $5 \% \mathrm{HCl}$ bath overnight prior to processing new samples. The digestion vessels and labware were twice rinsed with Evoqua ${ }^{\circledR} \mathrm{DI}_{2} \mathrm{O}$ and allowed to dry. Trace metal grade (Fisher Scientific, USA) acids and $>18 \mathrm{M} \Omega$ Evoqua ${ }^{\circledR}$ DI $\mathrm{H}_{2} \mathrm{O}$ were used to prepare all acid leaching baths.

\subsubsection{Extraction Chromatography for Strontium Isotope Ratio Analysis}

Separating $\mathrm{Rb}$ (and also other matrix interferences) from Sr prior to analysis was necessary because in samples with significant amounts of $\mathrm{Rb}$, higher abundance of ${ }^{87} \mathrm{Rb}$ leads to an isobaric interference on ${ }^{87} \mathrm{Sr}$ that cannot be resolved by the mass spectrometer and because SRM987, which was measured routinely to monitor drift in isotope ratios during the course of the measurement, does not contain $\mathrm{Rb}$.

Nitric acid was distilled once from concentrated ACS grade $\mathrm{HNO}_{3}$ in a Savillex DST-1000 sub-boiling still (Savillex, USA). The concentration of the acid after distillation was determined by volumetric titration against certified 0.1 and $1 \mathrm{M}$ sodium hydroxide solutions. Further acid dilutions were made gravimetrically using MQ water.

To achieve a small elution volume (approximately $2 \mathrm{~mL}$ ) and low levels of procedural blank, an extraction chromatography protocol was modified from a previously reported method for Sr isotope analysis of dust particles [79]. This procedure takes advantage of a commercially available resin for $\mathrm{Sr}$ separation $\left(4,4^{\prime}\left(5^{\prime}\right)\right.$-di-tbutylcyclohexano 18-crown-6 [crown ether] in 1-octanol, SR1ML-R50-S; Eichrom Technologies, USA). As reported by Horwitz et al. [80], the partition coefficient ( $\left.k^{\prime}\right)$ of

this resin is highest for ${ }^{88} \mathrm{Sr}$ in $\mathrm{HNO}_{3}$ concentrations that exceed $6 \mathrm{M}$, although the $k^{\prime}$ is 
reasonably high at the 5-6 $\mathrm{M} \mathrm{HNO}_{3}$ used in this study. In contrast, the resin shows no affinity for interfering cations such as $\mathrm{Ca}, \mathrm{Mg}$ and other trace elements at this acid concentration. At $0.01 \mathrm{M} \mathrm{HNO}_{3}$, the $k$ ' for Sr drops significantly below 1, allowing for quantitative separation of $\mathrm{Sr}$ from matrix elements.

To downscale the elution scheme from using a pre-packed $1 \mathrm{~mL}$ cartridge (SR1MLR50-S), approximately $0.04 \mathrm{~g}$ of the resin was slurry-packed onto the center column of a Luer-Lock male-female fitting (Cole-Parmer, USA). A custom-cut piece of Whatman 41 ashless filter (W-41; Sigma-Aldrich, USA) with an approximate diameter of $3 \mathrm{~mm}$ was used to retain the resin within the column reservoir. The packed micro-columns were placed on a vacuum box to control the load and elution flow rates.

Extraction chromatography was conducted inside a class-100 Microzone tracemetal workstation (DFMZ Inc, Canada). Prior to processing the samples on the resin, approximately $2 \mathrm{~mL}$ of the digested heroin at $1.87 \pm 0.31 \mathrm{M} \mathrm{HNO}_{3}$ was mixed with $1 \mathrm{~mL}$ of concentrated $\mathrm{HNO}_{3}(15-16 \mathrm{M})$ in $5 \mathrm{~mL}$ PPE centrifuge vials to raise the concentration of the load solution to greater than $5 \mathrm{M} \mathrm{HNO}_{3}$. Background $\mathrm{Sr}$ on the resin was removed by loading $2 \mathrm{~mL}$ of $0.01 \mathrm{M} \mathrm{HNO}_{3}$ and the columns were converted to the load solution by adding $1 \mathrm{~mL}$ of $6 \mathrm{M} \mathrm{HNO}_{3}$. Samples were subsequently loaded, and the matrix elements were removed by adding $1 \mathrm{~mL}$ of $6 \mathrm{M} \mathrm{HNO}_{3}$. Strontium was eluted in $1.9 \mathrm{~mL}$ of $0.01 \mathrm{M}$ $\mathrm{HNO}_{3}$ into $5 \mathrm{~mL}$ PPE centrifuge vials for measurement of $\mathrm{Sr}$ isotope ratios. The flow rate for load and elution of $\mathrm{Sr}$ was adjusted to less than $1 \mathrm{~mL} / \mathrm{min}$ to ensure quantitative separation. The resin was flushed from the micro-columns with MQ water and replaced with fresh resin for each sample. 


\subsubsection{High-precision Strontium Isotope Ratio Analysis}

The ${ }^{87} \mathrm{Sr} /{ }^{86} \mathrm{Sr}$ isotope ratio consists of the radiogenic isotope ${ }^{87} \mathrm{Sr}$ and the stable reference isotope ${ }^{86} \mathrm{Sr}$. The ratio of ${ }^{87} \mathrm{Sr} r{ }^{86} \mathrm{Sr}$ were measured in 198 heroin samples with $>1.25 \mu \mathrm{g} / \mathrm{g}$ [Sr] on a Neptune Plus (Thermo Fisher Scientific, Germany) multi-collector ICP mass spectrometer at the Neptune Isotope Lab (NIL) at the University of Miami. The instrument at NIL is equipped with 9 Faraday collectors that can be associated to eight $10^{11}$ $\Omega$, and two $10^{10} \Omega$ and $10^{12} \Omega$ amplifiers to extend the dynamic range of the measurements. A more detailed description of the instrumental configuration and the acquisition parameters can be found in Pourmand et al. [79].

The Faraday collectors and associated isotopes of $\mathrm{Kr}, \mathrm{Rb}$ and $\mathrm{Sr}$ are shown in Table 18. After loading the cup configuration for Sr isotopes, an automatic gain calibration of the Faraday collectors was carried out through the Neptune software and the instrumental parameters (sample, cooling and auxiliary gases, torch position and ion optics) were tuned to optimize for sensitivity and stability of the signal in a $100 \mathrm{ng} \mathrm{g}^{-1} \mathrm{Sr}$ solution. The sample and standard solutions were introduced into the plasma through an ESI $^{\circledR}$ Apex-Q desolvation nebulizer at a flow rate of $\sim 100 \mu \mathrm{L} \min ^{-1}$ via an autosampler controlled through the Neptune software.

Data acquisition involved five blocks of five cycles at $8.389 \mathrm{~s}$ integration time. Sample uptake was set to 70 seconds and baseline calibration was performed prior to each measurement through the Neptune software for $30 \mathrm{~s}$. The analytical precision reported on isotope ratios from this study was on the basis of $95 \%$ confidence intervals ( $2 \sigma$ mean) of individual measurements. 
Equation 4. Where $\mathrm{R}_{\text {true }}$ and $\mathrm{R}_{\text {measured }}$ are the accepted and measured isotopic ratios of masses $\mathrm{M}_{2}$ and $\mathrm{M}_{1}$, respectively. The mass bias coefficient, $\beta$, is a free parameter determined experimentally and applied to other measured isotope ratios for mass bias correction

$$
R_{\text {true }}=R_{\text {measured }}\left(\frac{M_{2}}{M_{1}}\right)^{\beta}
$$

Mass-dependent fractionation of heavy versus light isotopes of $\mathrm{Sr}$ that occurs in the plasma was characterized by internal normalization using the exponential law and the relationship shown in Equation 4[81]. Data reduction and adjustments for isobaric interferences were performed directly through the Neptune Method Editor software.

\begin{tabular}{|c|c|c|c|c|c|c|c|c|c|c|c|}
\hline \multicolumn{9}{|c|}{ Faraday detectors and corresponding isotopes } & \multirow{2}{*}{$\begin{array}{l}\text { Integration } \\
\text { Time (s) }\end{array}$} & \multirow[b]{2}{*}{ Block } & \multirow[b]{2}{*}{ Cycle } \\
\hline L4 & L3 & L2 & L1 & $\mathrm{C}$ & $\mathrm{H} 1$ & $\mathrm{H} 2$ & $\mathrm{H} 3$ & $\mathrm{H} 4$ & & & \\
\hline${ }^{82} \mathrm{Kr}$ & ${ }^{83} \mathrm{Kr}$ & ${ }^{84} \mathrm{Sr}$ & ${ }^{85} \mathrm{Rb}$ & ${ }^{86} \mathrm{Sr}$ & ${ }^{87} \mathrm{Sr}$ & ${ }^{88} \mathrm{Sr}$ & - & - & 8.389 & 5 & 10 \\
\hline
\end{tabular}

During the Sr isotope measurements (Table 18), the abundances of ${ }^{82} \mathrm{Kr},{ }^{83} \mathrm{Kr}$ and ${ }^{85} \mathrm{Rb}$ isotopes were also monitored. The contributions of ${ }^{84} \mathrm{Kr}$ and ${ }^{86} \mathrm{Kr}$ on ${ }^{84} \mathrm{Sr}$ and ${ }^{86} \mathrm{Sr}$ beams, respectively, were accounted for by measuring ${ }^{83} \mathrm{Kr}$ and allowing for abundance ratios ${ }^{83} \mathrm{Kr} /{ }^{84} \mathrm{Kr}=0.2017$ and ${ }^{83} \mathrm{Kr} /{ }^{86} \mathrm{Kr}=0.6647$. The influence of background $\mathrm{Kr}$ isotopes on Sr was accounted for by initiating on-peak-zero subtraction (OPZ) at the beginning of each measurement sequence, which rendered the $\mathrm{Kr}$ beam contribution to $\mathrm{Sr}$ isotopes negligible. The natural abundance ratio ${ }^{88} \mathrm{Sr} /{ }^{86} \mathrm{Sr}=8.375209$ was used to correct for the influence of instrumental mass bias on isotope ratios using Equation 4.

High-performance Jet sampler and X-series Ni skimmer cones were used to improve sensitivity. Every five sample measurements were bracketed with analysis of two 
SRM987 standard solutions at $100 \mu \mathrm{g} / \mathrm{L}$. The mean ${ }^{87} \mathrm{Sr} /{ }^{86} \mathrm{Sr}$ ratio in SRM987 measured during the course of this study was $0.710271 \pm 0.00002(n=22)$, which deviated from the accepted value of 0.710248 by $32 \mathrm{ppm}$ on average [82]. The measured ${ }^{87} \mathrm{Sr} /{ }^{86} \mathrm{Sr}$ ratios in the samples were corrected for mass bias and isobaric interferences and the final ratio was further adjusted relative to the accepted value of 0.710248 for SRM 987 to allow comparison with literature measurements of radiogenic Sr isotopes.

\subsubsection{Other Isotopic Systems}

The isotope systematics of boron and lead were investigated as well, however there were difficulties with these analytes as a result of the microwave digestion method. Fractionation of boron isotope composition was experienced because of the open-vessel nature of microwave-assisted acid digestion, as well as persistent boron contamination in reagents. Lead was not present in sufficient concentrations to be amenable for precise measurement of lead isotopic ratios. It was estimated that $<10 \%$ of the samples would have sufficient lead concentrations for isotope ratio measurement by the sample preparation method described above. More information on boron and lead isotope systematics are provided in Chapter 5.

\subsection{Results}

\subsubsection{Strontium Isotope Ratios}

To examine the influence of matrix elements on the $\mathrm{Sr}$ isotope ratios, 12 samples were processed by diluting the digestion solutions $2: 1$ to bring the solutions to approximately $0.6 \mathrm{M} \mathrm{HNO}_{3}$ and $[\mathrm{Sr}]$ into the ranges of $5-50 \mu \mathrm{g} / \mathrm{L}$ and analyzed directly for

${ }^{87} \mathrm{Sr} /{ }^{86} \mathrm{Sr}$. The results were compared to those from aliquots of the same samples processed 
through extraction chromatography before measurement. As shown in Figure 29, large discrepancies were observed between some samples that were measured by direct dilution and those where $\mathrm{Sr}$ was separated from interfering elements. The concentrations of elemental $\mathrm{Sr}$ and $\mathrm{Rb}$ had been previously determined for these samples, and some were as high as $33 \%$ relative abundance of $\mathrm{Rb} / \mathrm{Sr}$. These results demonstrated the need for extraction chromatography to achieve accurate measurements of ${ }^{87} \mathrm{Sr} /{ }^{86} \mathrm{Sr}$ in illicit heroin samples.

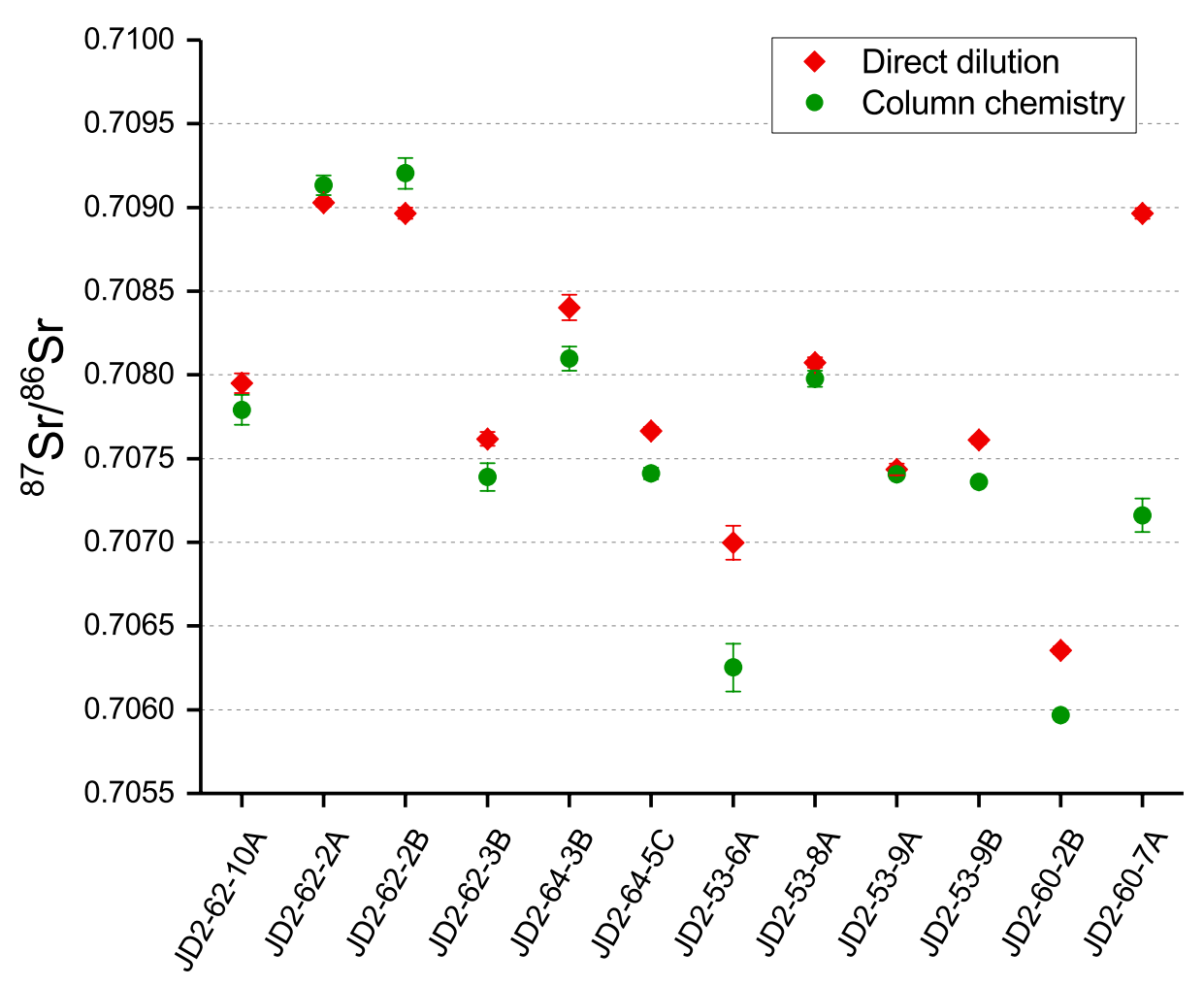

Figure 29. Effect of column chemistry (green circles), compared to direct dilution (red diamonds) on the measured values of ${ }^{87} \mathrm{Sr} /{ }^{86} \mathrm{Sr}$ in 12 authentic heroin samples. Samples JD2-62-2A/B and JD2-53-9A/B are pairs of duplicate digestions of two different heroin samples. 


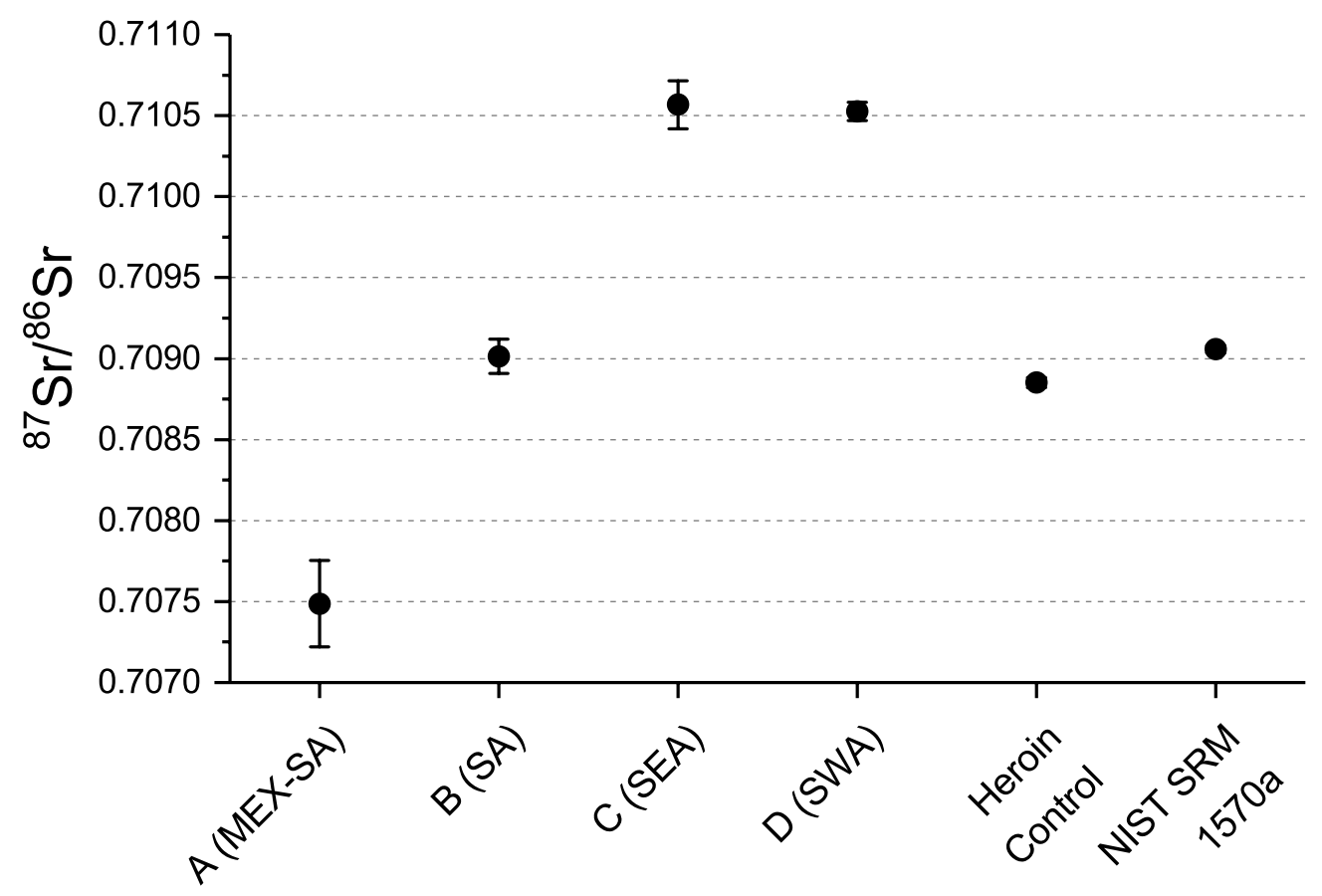

Figure 30. Means and uncertainty of ${ }^{87} \mathrm{Sr} /{ }^{86} \mathrm{Sr}$ isotope ratios for powder heroin samples A-D (arbitrary ID assignment), heroin duplicate standard and NIST SRM 1570a, where all samples were separately digested, prepared and analyzed. Error bars are shown for all samples but are too small to be seen for the heroin duplicate standard and for NIST SRM 1570a. $\mathrm{N}=3$ for all samples except for C (SEA), where $\mathrm{N}=4$.

The fidelity of Sr isotope measurements at NIL was previously shown by analyzing geological reference materials [83]. There were no assumptions made regarding sample homogeneity. Because of the controlled substance status of the heroin samples and averting the risk of external and cross-contamination, there were no attempts made to homogenize the samples prior to digestion. One sample from each region, as well as the heroin control sample and NIST SRM 1570a, were analyzed in triplicate. The samples were all separately digested and individually processed by column chemistry for strontium isolation to account for method reproducibility. The means and $95 \%$ confidence intervals of the replicate digestions are shown in Figure 30. The NIST SRM 1570a and heroin control sample 
showed consistent ${ }^{87} \mathrm{Sr} /{ }^{86} \mathrm{Sr}$ and therefore good method reproducibility (the errors were so small the error bars cannot be seen). The variation observed in the heroin samples A, B, C and $\mathrm{D}$ was believed to have been caused by sample heterogeneity. This is also the first reported value of ${ }^{87} \mathrm{Sr} /{ }^{86} \mathrm{Sr}$ for NIST SRM 1570a, trace elements in spinach leaves $(0.70905$ $\pm 0.00002, \mathrm{n}=3,95 \%$ confidence interval).

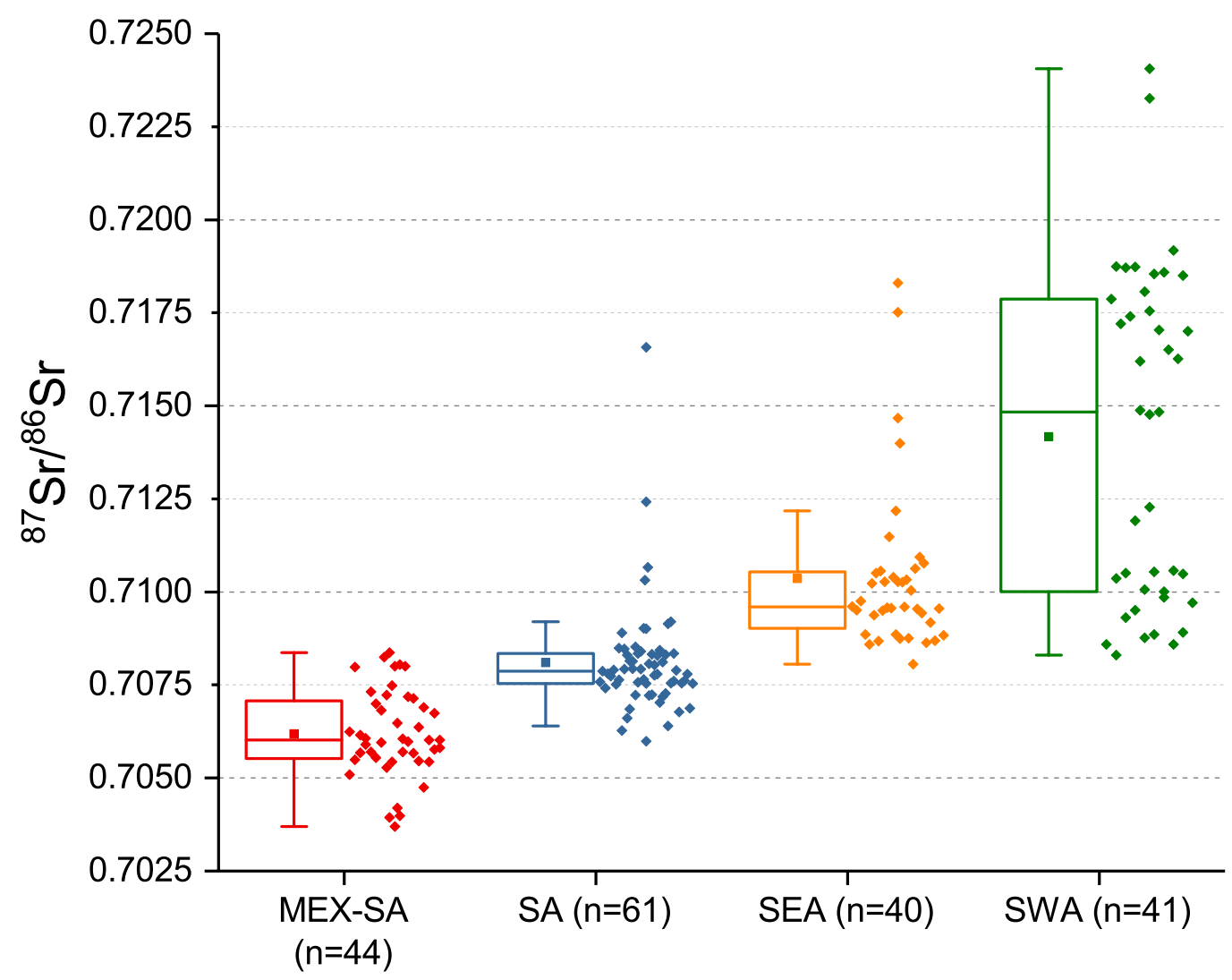

Figure 31. Box and whisker plot of the distributions and group means of ${ }^{87} \mathrm{Sr} /{ }^{86} \mathrm{Sr}$ isotope ratios for powder heroin

The measured ratio of ${ }^{87} \mathrm{Sr} /{ }^{86} \mathrm{Sr}$ of illicit heroin samples is shown in Figure 31, grouped by the four major heroin-producing regions. The ${ }^{87} \mathrm{Sr} /{ }^{86} \mathrm{Sr}$ values of heroin samples were averaged and are reported at 95\% confidence levels. Samples from MEX-SA had a 
mean of $0.70619 \pm 0.00035$, (range: $0.70370-0.70837$ ). SA heroin samples had a mean of $0.70810 \pm 0.00038$ (range: 0.70599 to 0.71658 ), although $90 \%$ of the values were below 0.70912. The heroin samples from SEA had a mean of $0.71036 \pm 0.00070$ (range: 0.70806 to 0.71830 ). While the groups of MEX-SA, SA and SEA had data that were distributed tightly surrounding their means, there were actually very few SWA samples with ${ }^{87} \mathrm{Sr} r{ }^{86} \mathrm{Sr}$ values near to the calculated mean $(0.71417 \pm 0.00141$, range: $0.70831-0.72405)$. The mean of the SWA group was not as well defined as compared to MEX-SA, SA and SEA samples; analysis of the data indicated this may have been the results of subgrouping and non-normality within distribution of ${ }^{87} \mathrm{Sr} /{ }^{86} \mathrm{Sr}$ values from the SWA group.

Obtaining additional intelligence information about these samples may reveal that they represent subgroups and may allow for discrimination between different countries within SWA (e.g., Afghanistan and Iran).

\subsubsection{Evaluation of Predictive Potential}

In addition to characterizing the ${ }^{87} \mathrm{Sr} /{ }^{86} \mathrm{Sr}$ values for the heroin sample from the four geographic regions, the rate of accuracy in predicting provenance was estimated by comparing the ${ }^{87} \mathrm{Sr} /{ }^{86} \mathrm{Sr}$ values of the authentic heroin samples to the mean values of each group. The results are shown in Table 19. Heroin samples of groups MEX-SA and SA were correctly classified $76 \%$ and $81 \%$ of the time, respectively. From Figure 31 , it can be seen that MEX-SA and SA have some overlap in their sample distribution and it was not surprising that a few samples from these groups would be misclassified because their ${ }^{87} \mathrm{Sr} /{ }^{86} \mathrm{Sr}$ values were closer to the mean of the other group. There were also a few SA samples which had much higher than average ${ }^{87} \mathrm{Sr} /{ }^{86} \mathrm{Sr}$ values and were misclassified as 
SEA and SWA. Considering the additional groups of Southeast and Southwest Asia, an overall correct classification rate of $71 \%$ was estimated for unknown heroin samples.

Table 19. Prediction of Heroin Origin by logistic regression of ${ }^{87} \mathrm{Sr} /{ }^{86} \mathrm{Sr}$ isotope ratios

\begin{tabular}{|c|c|c|c|c|c|}
\hline \multirow[b]{2}{*}{ Actual Origin } & \multicolumn{5}{|c|}{ Predicted Count for Training Set $(n=121)$} \\
\hline & MEX-SA & SA & SEA & SWA & $\%$ Correct \\
\hline MEX-SA $(n=26)$ & 20 & 6 & 0 & 0 & $77 \%$ \\
\hline $\mathrm{SA}(\mathrm{n}=36)$ & 9 & 24 & 2 & 1 & $67 \%$ \\
\hline $\operatorname{SEA}(n=30)$ & 0 & 10 & 16 & 4 & $53 \%$ \\
\hline SWA $(n=29)$ & 0 & 1 & 8 & 20 & $69 \%$ \\
\hline $\operatorname{SUM}(n=121)$ & 29 & 41 & 26 & 25 & $66 \%$ \\
\hline \multirow[t]{2}{*}{$\%$ Confusion } & $31 \%$ & $41 \%$ & $38 \%$ & $20 \%$ & \\
\hline & \multicolumn{5}{|c|}{ Predicted Count for Test Set $(n=77)$} \\
\hline Actual Origin & MEX-SA & SA & SEA & SWA & $\%$ Correct \\
\hline MEX-SA $(n=24)$ & 20 & 4 & 0 & 0 & $83 \%$ \\
\hline $\mathrm{SA}(\mathrm{n}=31)$ & 4 & 26 & 1 & 0 & $84 \%$ \\
\hline $\operatorname{SEA}(n=11)$ & 0 & 1 & 10 & 0 & $91 \%$ \\
\hline SWA $(n=11)$ & 0 & 5 & 4 & 2 & $18 \%$ \\
\hline SUM (n=77) & 24 & 36 & 15 & 2 & $75 \%$ \\
\hline \multirow[t]{2}{*}{$\%$ Confusion } & $17 \%$ & $28 \%$ & $33 \%$ & $0 \%$ & \\
\hline & \multicolumn{5}{|c|}{ Predicted Count for Combined Data $(n=198)$} \\
\hline Actual Origin & MEX-SA & SA & SEA & SWA & $\%$ Correct \\
\hline MEX-SA $(n=50)$ & 38 & 12 & 0 & 0 & $76 \%$ \\
\hline SA $(n=67)$ & 9 & 54 & 2 & 2 & $81 \%$ \\
\hline $\operatorname{SEA}(n=41)$ & 0 & 11 & 26 & 4 & $63 \%$ \\
\hline SWA $(n=40)$ & 0 & 5 & 13 & 22 & $55 \%$ \\
\hline $\operatorname{SUM}(n=198)$ & 47 & 82 & 41 & 28 & $71 \%$ \\
\hline$\%$ Confusion & $19 \%$ & $34 \%$ & $37 \%$ & $21 \%$ & \\
\hline
\end{tabular}

The prediction accuracy of the authentic samples from SEA and SWA was complicated by overlap of their samples ${ }^{87} \mathrm{Sr} /{ }^{86} \mathrm{Sr}$ values. There were $11 \mathrm{SEA}$ samples with lower than average ${ }^{87} \mathrm{Sr} /{ }^{86} \mathrm{Sr}$ values that fell closer to the mean of SA and so were 
misclassified as SA. In addition, the SEA samples with very high ${ }^{87} \mathrm{Sr} /{ }^{86} \mathrm{Sr}$ values and were misclassified as SWA. Of the SWA samples, 18 had ${ }^{87} \mathrm{Sr} /{ }^{86} \mathrm{Sr}$ values that overlapped with the samples of SA and SEA. As mentioned previously, from Figure 31, the SWA data appear to exist in multiple clusters, unlike the data from the other regions. If the apparent sub-groups of SWA were treated separately, the lower cluster of 18 samples would still be confused as SA and SEA and the mean ${ }^{87} \mathrm{Sr} /{ }^{86} \mathrm{Sr}$ ratio for the other SWA samples would be shifted to a higher value, which would be distinguishable from the other regions.

\subsection{Merging Elemental Data with ${ }^{87} \mathrm{Sr} /{ }^{86} \mathrm{Sr}$ Isotope Ratios}

The work performed in Chapter 2 produced a multivariate model of analysis based upon the quantitative analysis of nine elements. None of the nine elements, on their own quantitative values, had the discrimination power of the ${ }^{87} \mathrm{Sr} /{ }^{86} \mathrm{Sr}$ radiogenic isotope ratio systematic. A log transform of the elemental concentration $(\mu \mathrm{g} / \mathrm{g})$ of the elements ${ }^{23} \mathrm{Na}$, ${ }^{24} \mathrm{Mg},{ }^{52} \mathrm{Cr},{ }^{57} \mathrm{Fe},{ }^{66} \mathrm{Zn},{ }^{90} \mathrm{Zr},{ }^{111} \mathrm{Cd},{ }^{208} \mathrm{~Pb}$ and ${ }^{238} \mathrm{U}$ have shown the potential to correctly associate $88 \%$ of unknown heroin samples to their region of origin. It was expected that combining the data of the most useful elements with that of the ${ }^{87} \mathrm{Sr} /{ }^{86} \mathrm{Sr}$ would further improve the model's performance, being that the radiogenic strontium isotope ratio as a single data point was able to correctly assign $75 \%$ of the unknown samples.

The radiogenic isotope ratio of strontium performed relatively poorly for SWA samples with regard to correct assignment, because of the apparently bimodal distribution of the data from that region. It would be useful if the inclusion of elemental data into a prediction model, which performed well for classification of SWA samples, would make up for the challenges of ${ }^{87} \mathrm{Sr} /{ }^{86} \mathrm{Sr}$ in determining provenance for the SWA samples. 
Therefore, a fusion of elemental quantitative data and ${ }^{87} \mathrm{Sr} /{ }^{86} \mathrm{Sr}$ data was desired to see if the two data sets could supplement one another and produce a higher rate of correct provenance determination than either could separately.

Table 20. Prediction of Origin by linear discriminant analysis by $\mathrm{LOG}_{10}\left({ }^{23} \mathrm{Na},{ }^{52} \mathrm{Cr}\right.$, ${ }^{66} \mathrm{Zn},{ }^{90} \mathrm{Zr},{ }^{111} \mathrm{Cd},{ }^{238} \mathrm{U}$ ) and ${ }^{87} \mathrm{Sr} /{ }^{86} \mathrm{Sr}$ isotope ratios.

\begin{tabular}{|c|c|c|c|c|c|}
\hline \multirow[b]{2}{*}{ Actual Origin } & \multicolumn{5}{|c|}{ Predicted Count for Training Set } \\
\hline & MEX-SA & SA & SEA & SWA & $\%$ Correct \\
\hline MEX-SA $(n=26)$ & 25 & 1 & 0 & 0 & $96 \%$ \\
\hline SA $(n=36)$ & 2 & 33 & 0 & 1 & $92 \%$ \\
\hline SEA $(n=30)$ & 0 & 0 & 30 & 0 & $100 \%$ \\
\hline SWA $(n=29)$ & 1 & 0 & 0 & 28 & $97 \%$ \\
\hline $\operatorname{SUM}(n=121)$ & 28 & 34 & 30 & 29 & $96 \%$ \\
\hline \multirow[t]{2}{*}{$\%$ Confusion } & $11 \%$ & $3 \%$ & $0 \%$ & $3 \%$ & \\
\hline & \multicolumn{5}{|c|}{ Predicted Count for Test Set } \\
\hline Actual Origin & MEX-SA & SA & SEA & SWA & $\%$ Correct \\
\hline $\operatorname{MEX}-S A(n=24)$ & 19 & 5 & 0 & 0 & $79 \%$ \\
\hline $\mathrm{SA}(\mathrm{n}=31)$ & 2 & 29 & 0 & 0 & $94 \%$ \\
\hline SEA $(n=11)$ & 0 & 0 & 11 & 0 & $100 \%$ \\
\hline SWA $(n=11)$ & 0 & 1 & 1 & 9 & $82 \%$ \\
\hline SUM $(n=77)$ & 21 & 35 & 12 & 9 & $88 \%$ \\
\hline \multirow[t]{2}{*}{$\%$ Confusion } & $10 \%$ & $17 \%$ & $8 \%$ & $0 \%$ & \\
\hline & \multicolumn{5}{|c|}{ Predicted Count for Combined Data } \\
\hline Actual Origin & MEX-SA & SA & SEA & SWA & $\%$ Correct \\
\hline MEX-SA $(n=50)$ & 45 & 5 & 0 & 0 & $90 \%$ \\
\hline SA $(n=67)$ & 5 & 61 & 0 & 1 & $91 \%$ \\
\hline SEA $(n=41)$ & 0 & 0 & 41 & 0 & $100 \%$ \\
\hline SWA $(n=40)$ & 1 & 0 & 1 & 38 & $95 \%$ \\
\hline SUM $(n=198)$ & 51 & 66 & 42 & 39 & $93 \%$ \\
\hline$\%$ Confusion & $12 \%$ & $8 \%$ & $2 \%$ & $3 \%$ & \\
\hline
\end{tabular}

Because the data set was limited to $\geq 40$ samples per group by the availability of ${ }^{87} \mathrm{Sr} /{ }^{86} \mathrm{Sr}$ isotope ratio data, the elemental data to be combined was reduced to the most informative 6 elements. A stepwise selection of the most useful 6 variables out of the included elements from Chapter 2 was made on the basis of the highest $\mathrm{F}$ ratio from the 
remaining variables after each selection. The F ratio is an indication of the variable's discriminatory power with respect to the assignment of region, in this case [84]. Table 20 shows the correct associations as confusion rates when $\mathrm{LOG}_{10}\left({ }^{23} \mathrm{Na},{ }^{52} \mathrm{Cr},{ }^{66} \mathrm{Zn},{ }^{90} \mathrm{Zr},{ }^{111} \mathrm{Cd}\right.$, $\left.{ }^{238} \mathrm{U}\right)$ and ${ }^{87} \mathrm{Sr} /{ }^{86} \mathrm{Sr}$ data are used together in a linear discriminant model for provenance determination. For the unknown test samples, the model performed $88 \%$, however the number of samples for which ${ }^{87} \mathrm{Sr} /{ }^{86} \mathrm{Sr}$ data were available limited the size of both the training as well as the testing set. When using the entire data set of samples with ${ }^{87} \mathrm{Sr} /{ }^{86} \mathrm{Sr}$ data available, the prediction rates increased to $93 \%$. Each region was correctly assigned $\geq 90 \%$ of the time.

Table 21. Canonical coefficients of linear discriminant analysis by $\mathrm{LOG}_{10}\left({ }^{23} \mathrm{Na},{ }^{52} \mathrm{Cr}\right.$, $\left.{ }^{66} \mathrm{Zn},{ }^{90} \mathrm{Zr},{ }^{111} \mathrm{Cd},{ }^{238} \mathrm{U}\right)$ and ${ }^{87} \mathrm{Sr} /{ }^{86} \mathrm{Sr}$ isotope ratios.

\begin{tabular}{|c|c|c|c|}
\hline Variable & Canon1 & Canon2 & Canon3 \\
\hline${ }^{87} \mathrm{Sr} /{ }^{86} \mathrm{Sr}$ & -0.6212 & 0.4871 & 0.4754 \\
\hline${ }^{23} \mathrm{Na}$ & 0.9067 & -0.1372 & 0.0590 \\
\hline${ }^{52} \mathrm{Cr}$ & 0.4200 & 0.5892 & 0.1991 \\
\hline${ }^{66} \mathrm{Zn}$ & -0.6142 & 0.0089 & -0.0080 \\
\hline${ }^{90} \mathrm{Zr}$ & 0.7269 & -0.1994 & 0.4479 \\
\hline${ }^{111} \mathrm{Cd}$ & -0.5780 & -0.3656 & 0.5788 \\
\hline${ }^{238} \mathrm{U}$ & 0.0509 & 0.7310 & 0.4008 \\
\hline
\end{tabular}

The canonical coefficients of the LDA model are shown on Table 21. The canonical coefficients are multiplied by the data of each variable for all samples to define the position of the sample within, in this case, three-dimensional space. The length of the sample's vector in the $\mathrm{x}, \mathrm{y}$ and $\mathrm{z}$ dimensions are defined by the linear combination of Canon 1, 2 and 3 coefficient and the sample's value for each variable. The assignment of provenance is made by measuring the shortest vector distance of the sample's data point to that of the 
group means of MEX-SA, SA, SEA and SWA. The canonical scores of all data points are shown in a 3D scatter plot on Figure 32.

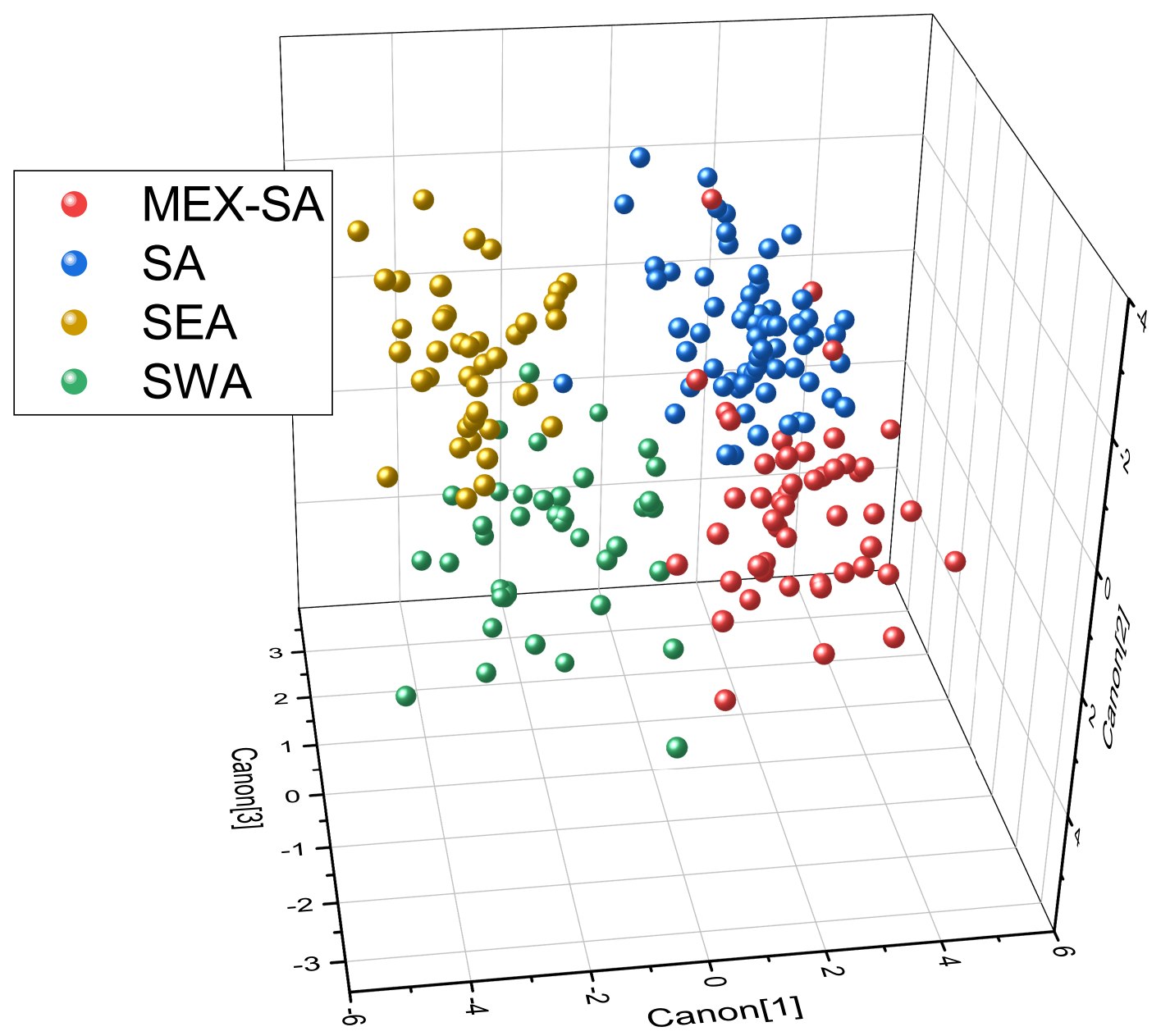

Figure 32. 3D scatter plot of canonical score of 198 samples for which $\mathrm{LOG}_{10}\left({ }^{23} \mathrm{Na},{ }^{52} \mathrm{Cr},{ }^{66} \mathrm{Zn},{ }^{90} \mathrm{Zr},{ }^{111} \mathrm{Cd}\right.$, ${ }^{238} \mathrm{U}$ ) and ${ }^{87} \mathrm{Sr} /{ }^{86} \mathrm{Sr}$ data are available. MEX-SA is shown in red, SA in blue, SEA in gold and SWA in green.

\subsection{Conclusion}

This work presents the first reported use of strontium isotope ratio analysis for the geographic sourcing of 186 illicit heroin samples of known origin. A microwave-assisted 
acid digestion method is described for the heroin matrix and methods for micro-column purification and strontium ratio measurements by MC-ICP-MS are also reported.

The fact that the ${ }^{87} \mathrm{Sr} /{ }^{86} \mathrm{Sr}$ ratios showed 77 to $82 \%$ discrimination between South American and SA-like Mexican heroin suggests that whether the elemental strontium is biologically available from the opium or introduced by external contamination, it carries a characteristic radiogenic strontium isotope ratio associated with a geographic location. Considering the additional groups of Southeast and Southwest Asia, an overall correct classification rate of approximately $70 \%$ was estimated for unknown heroin samples.

Data resulting from the work described in Chapter 2 and Chapter 4 of this dissertation includes over 200 heroin samples for which elemental quantitative data as well as isotope ratio data were available. Details on the methods of preparation and analysis of those samples can be found in Chapters 2 and 4. The most useful set of nine elements ( $\log _{10}$ transformed $\mu \mathrm{g} / \mathrm{g}$ concentration) is described in Chapter 2 and correctly assigns heroin samples to the region from which it originated at an overall rate of $89 \%$ and no worse than $85 \%$ of the time for any particular region.

The isotope ratio of radiogenic strontium ${ }^{87} \mathrm{Sr} /{ }^{86} \mathrm{Sr}$ correctly predicted the origin of $75 \%$ of "blind" heroin samples based upon a univariate logistic regression. Heroin originating from MEX-SA, SA and SEA regions were correctly classified over $83 \%$ of the time. The main difficultly was with SWA samples, which could be overcome by combining the elemental data from Chapter 2 with the ${ }^{87} \mathrm{Sr} /{ }^{86} \mathrm{Sr}$ data. The performance of a multivariate model of $\mathrm{LOG}_{10}\left({ }^{23} \mathrm{Na},{ }^{52} \mathrm{Cr},{ }^{66} \mathrm{Zn},{ }^{90} \mathrm{Zr},{ }^{111} \mathrm{Cd},{ }^{238} \mathrm{U}\right)$ and ${ }^{87} \mathrm{Sr} /{ }^{86} \mathrm{Sr}$ isotope ratios correctly assigned $82 \%$ of unknown SWA samples and $88 \%$ of all unknown samples. Using 
a complete data set, $93 \%$ of all heroin samples could be correctly assigned using the ideal combination of data of $\mathrm{LOG}_{10}\left({ }^{23} \mathrm{Na},{ }^{52} \mathrm{Cr},{ }^{66} \mathrm{Zn},{ }^{90} \mathrm{Zr},{ }^{111} \mathrm{Cd},{ }^{238} \mathrm{U}\right)$ and ${ }^{87} \mathrm{Sr} /{ }^{86} \mathrm{Sr}$.

In summation, the ${ }^{87} \mathrm{Sr} /{ }^{86} \mathrm{Sr}$ results show promise for isotopic ratio analysis of strontium as a profiling technique of illicit heroin samples for geographic sourcing and perhaps, other intelligence purposes. When combined with the 6 of the elements from Chapter 2 , the profiling potential greatly increases to $93 \%$ overall with no region $<90 \%$ correctly associated. Whereas the elemental data experienced difficultly differentiating some samples of MEX-SA \& SA and the ${ }^{87} \mathrm{Sr} /{ }^{86} \mathrm{Sr}$ confused some SWA samples, the combination of both elemental quantitative data, $\mathrm{LOG}_{10}\left({ }^{23} \mathrm{Na},{ }^{52} \mathrm{Cr},{ }^{66} \mathrm{Zn},{ }^{90} \mathrm{Zr},{ }^{111} \mathrm{Cd}\right.$, ${ }^{238} \mathrm{U}$ ), and ${ }^{87} \mathrm{Sr} /{ }^{86} \mathrm{Sr}$ data into a single model minimized the misclassification of samples from those regions. 


\section{CHAPTER 5. ADDITIONAL ANALYSIS FOR HEROIN PROFILING}

The following chapter describes additional methods developed for chemical profiling of heroin that are thus far unreported in literature. The techniques outlined in this chapter had reasonable hypotheses for delivering successful results for profiling heroin based upon their origin. In this chapter will be reported the background, method details and results on the study of isotope ratio analysis of boron $\left(\delta{ }^{11} \mathrm{~B}\right)$, the contributions of adulterants to the elemental profile of street-level heroin samples, the volatile organic compounds (VOCs) and semi volatile organic compounds (SVOCs) associated with heroin samples and the fusion of ${ }^{87} \mathrm{Sr} /{ }^{86} \mathrm{Sr}$ isotope data with elemental quantitative data for provenance determination of heroin samples.

Because the systematics of $\delta^{11} \mathrm{~B}$ and VOCs were unproven for profiling heroin and limited in scope relative to the trace element study, the analysis was performed upon a smaller subset of the total sample size to not consume valuable sample material provided by the US Drug Enforcement Administration. For $\delta^{11} \mathrm{~B}$ analysis approximately 40 samples were chosen for analysis with $\mathrm{n} \geq 8$ samples from each of the four heroin-producing regions, MEX-SA, SA, SEA and SWA. Each of the samples had previously been characterized by both trace element quantification as well as ${ }^{87} \mathrm{Sr} /{ }^{86} \mathrm{Sr}$ isotope ratios, therefore the data from those techniques could be combined if doing so proved helpful to characterizing the heroin samples.

The study of VOCs (and SVOCs) was performed on four samples in total with 1 sample represented from each of the four groups. The analysis by $\mathrm{CMV}-\mathrm{GC} / \mathrm{MS}$ was more time-consuming and non-destructive and therefore required a greater level of sample 
security. As opposed to acid digestion, which could be initiated for 10 or more samples in less than one hour, the entire sampling and analytical procedure for CMV-GC-MS required approximately one hour for a single sample to be equilibrated, sampled and analyzed (per available GC-MS instrument). As a result, the purpose of the preliminary study was to demonstrate the utility of headspace compound detection by CMV-GCMS technique rather than confirming the ability to profile heroin; to do so would have required a working environment with a level of security greater than is likely to be found in an academic research laboratory.

\subsection{Boron Isotope Ratio Analysis, $\delta{ }^{11} \mathrm{~B}$}

\subsubsection{Background and Hypothesis}

Unlike for many isotope systems, there is a large relative mass difference, $(10 \%)$ in the two naturally-occurring boron isotopes, ${ }^{10} \mathrm{~B}$ and ${ }^{11} \mathrm{~B}$. For example, the other isotope systematic studied in this dissertation, ${ }^{87} \mathrm{Sr} /{ }^{86} \mathrm{Sr}$, has only a $1.2 \%$ mass difference in the two isotopes being measured. However, the ratio of ${ }^{87} \mathrm{Sr} /{ }^{86} \mathrm{Sr}$ changes because of the abundance of $\mathrm{Rb}$ in the geological material and the age because ${ }^{87} \mathrm{Sr}$ is radiogenic isotope produced through beta decay of ${ }^{87} \mathrm{Rb}$. On the other hand, variation in ${ }^{11} \mathrm{~B} /{ }^{10} \mathrm{~B}$ are as a result of their differences in geochemical reactivity, as the isotopes of boron are neither radioactive nor radiogenic.

The main challenges of analysis of boron isotope determination are isotopic fractionation during sample digestion, through volatilization, and imprecise measurements because of low sample concentration of boron [85]. In an acidic system, the boric acid molecule, $\mathrm{B}(\mathrm{OH})_{3}$, is favored over the tetrahydroxyborate weak base, $\mathrm{B}(\mathrm{OH})_{4}^{-}$. It is 
reported that the heavier isotope, ${ }^{11} \mathrm{~B}$, favors the boric acid species while the lighter, ${ }^{10} \mathrm{~B}$ favors the borate weak base [86]. Careful method development of a method for sample preparation to control this fractionation is likely the greatest obstacle to accurate boron isotope analysis.

\subsubsection{Preliminary Investigation}

In spite of the use of boron-free filter on the $\mathrm{DI} \mathrm{H}_{2} \mathrm{O}$ system and using Optimagrade acids, there was still challenges with boron contamination of method blanks and reagent blanks. Usually the background subtraction was an adequate means of addressing the background levels for quantitative analysis. As a particular obstacle for reliable isotope analysis, it was observed that boron isotopes were experiencing fractionation during the micro-wave assisted digestion process, as evident on Figure 33. Three replicates digestions were performed of NIST SRM 951a boric acid standard (NIST, USA) in the microwave and one using a closed vessel method in PFA vessels.

The results of these analyses demonstrated that the closed vessel method showed no deviation from the accepted values for $\delta{ }^{11} \mathrm{~B}$, while the microwave-assisted samples showed per-mille differences. The microwave digestion oven, as a safety feature, allows for sample venting in the event of over-pressurization. Additionally, the quartz microvessels have vented caps that all for pressure equalization, as well as the escape of vaporized sample, into the chamber of the main vessel itself. The vaporization and loss of sample was observed to favor ${ }^{11} \mathrm{~B}$, which led to greater than expected $\delta{ }^{11} \mathrm{~B}$ values in the resulting solution that was collected following the microwave-assisted acid digestion as 
shown in Figure 33. The values for the $\delta{ }^{11} \mathrm{~B}$ deviations were inconsistent such that they could not have been systematically corrected.

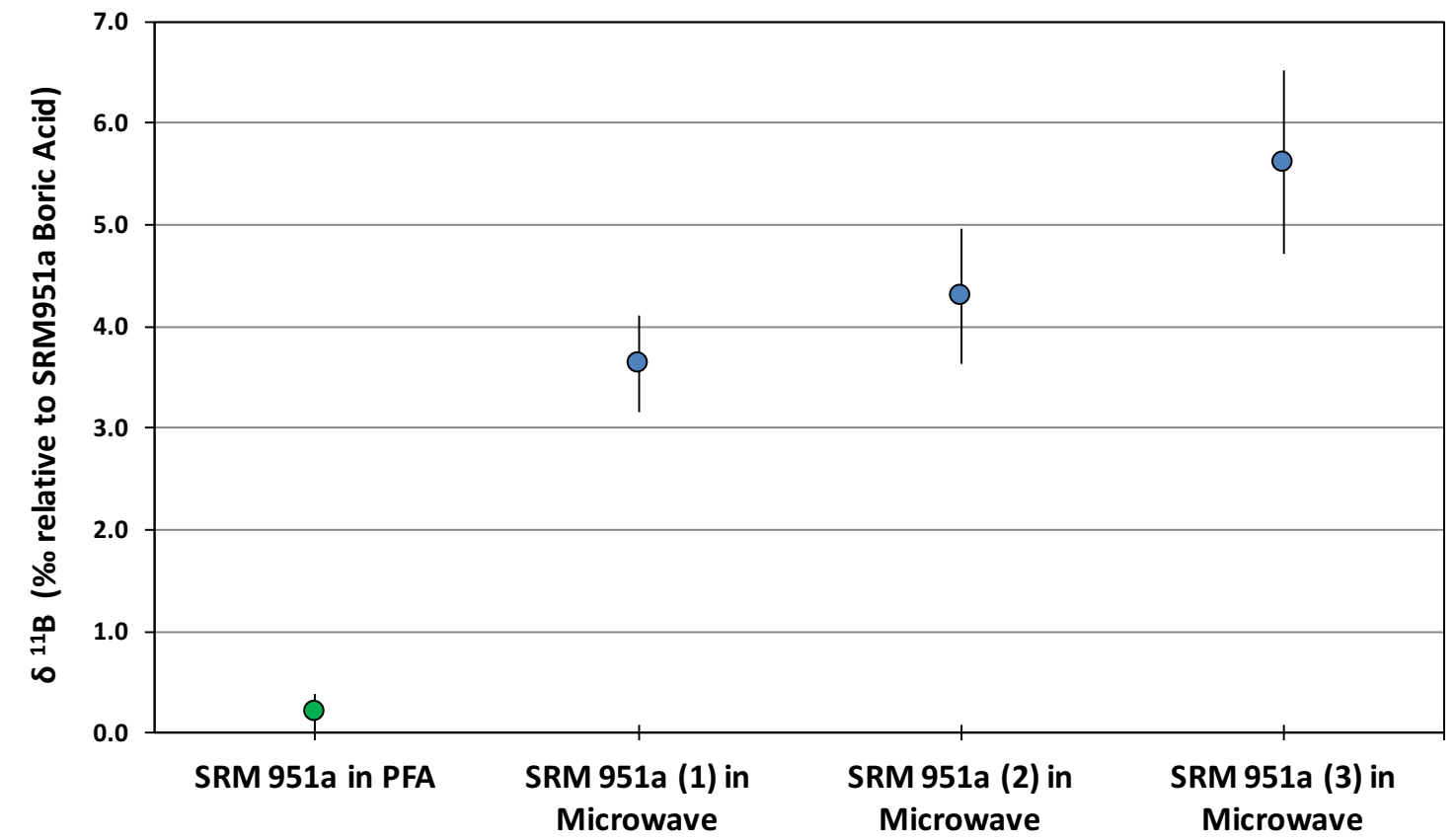

Figure 33: Observing fractionation of boron isotopes using microwave assisted-acid digestion (blue dots) as compared to a closed vessel digestion method (PFA; green dots).

It was determined that the results precluded boron isotope analysis on the basis that accurate and reproducible measurements could not be performed with the same sample preparation method as we had developed for the elemental analysis. Therefore, the method of hotplate digestion in sealed PFA, described above, was adopted for processing samples for analysis of $\delta{ }^{11} \mathrm{~B}$. Resources were also limited in the analysis that could be performed at NIL and Sr isotopic analysis had shown greater promise for success with respect to geographic profiling.

For these reasons and because having a high-throughput method for sample preparation was crucial for the large scale of analysis of samples for database creation, it 
was agreed that boron isotope analysis would only be performed for a limited number of samples to evaluate the usefulness of the technique for the purpose of profiling heroin. Only samples which had been analyzed for strontium isotope ratios ${ }^{87} \mathrm{Sr} /{ }^{86} \mathrm{Sr}$ and composed of $>10 \mu \mathrm{g} / \mathrm{g}$ boron (previously quantified by Q-ICP-MS) were attempted for $\delta^{11} \mathrm{~B}$ analysis. The fact that quantitative analysis of boron had already been performed on the samples was advantageous. As such, it was not expected that low sample concentrations would negatively affect the analytical precision of the boron isotope measurement.

One of the earliest reports of employing closed-vessel, microwave-assisted acid digestion for the inorganic analysis of SWA heroin samples was published in 2002 by Bora et al. wherein ten elements in total were analyzed quantitatively by electrothermal atomic absorption spectroscopy ( $\mathrm{Cd}$ and $\mathrm{Pb}$ ) and ICP-AES (Al, Ba, $\mathrm{Ca}, \mathrm{Cu}, \mathrm{Fe}, \mathrm{Mg}, \mathrm{Mn}$ and $\mathrm{Zn}$ ) [36]. The authors reported calcium as the element of highest abundance, which was supported by the results of this research for SWA samples, as well as SEA samples (subchapter 2.4.5), presumably as a result of the employment of lime in the reduction of morphine to morphine base [36].

\subsubsection{Methods and Materials}

As described in the previous section, the method of microwave-assisted acid digestion proved to cause irreproducible fractionation in the isotope abundance of ${ }^{11} \mathrm{~B} /{ }^{10} \mathrm{~B}$. Despite the microwave vessels themselves were closed, venting of gases was designed in the micro-insert vessels that were used to reduce acid consumption, lower sample mass requirements and increase sample through-put (see Methods sections in Chapter 2-4 beginning on pages 29 and 107). Therefore, a method that did not allow for vapors to escape 
from solution was desirable. To do so, closed-vessel digestion in concentrated, Optimagrade nitric acid (Fisher Scientific, USA) was performed in 4 mL PFA vessels (Savillex, USA) at sub-boiling temperature of $90^{\circ} \mathrm{C}$ for $24-28$ hours on a hot plate followed by chilling the vessels on ice. The boiling point of a commercial solution of $68 \% \mathrm{HNO}_{3}$ is $\sim 121^{\circ} \mathrm{C}[87]$.

A subset of 42 heroin samples ( $\mathrm{n} \geq 8$ from each region) were chosen for $\delta{ }^{11} \mathrm{~B}$ analysis. Sample masses ranged from $80 \mathrm{mg}$ to $200 \mathrm{mg}$ depending upon sample concentration, availability and density. Samples were weighed by difference directly into $6 \mathrm{~mL}$ PFA digestion vessels along with their lids. To the vessels was added $2 \mathrm{~mL}$ of Optima-grade Nitric Acid (Fischer Scientific). The lids were tightened using two plastic wrenches (one on the vessel body and one on the lid, torqued in opposing directions) such that there would not be escape of liquid or gases during digestion. The gross mass of the heroin, acid and closed vessel was recorded and compared to the post-digestion mass to evaluate if there was any significant loss of mass.

The digestion was accomplished by first sonicating the vessels for 30 minutes in DI $\mathrm{H}_{2} \mathrm{O}$. The vessels were shaken vigorously by hand to rinse any undissolved solid material off of the inner walls. The vessels were then sonicated for an additional 30 minutes in $\mathrm{DI} \mathrm{H}_{2} \mathrm{O}$. The outer walls of the vessels were dried and subsequently placed onto an Isotemp (Fisher Scientific, USA) heating block with a digital temperature control. A handheld thermocouple was used to find the areas on the surface of the heating mantle where the surface temperature was constant and most accurate to the desired temperature of $80^{\circ} \mathrm{C}$, which required the hot-block to be set at $85^{\circ} \mathrm{C}$. A slightly higher setting was 
necessary to achieve the desired surface temperature. The vessels were allowed to remain on the hotplate for at least 24 hours.

After the digestion had proceeded for 24 hours or longer, the vessels placed directly into ice to sublimate the accumulated vapors back into solution. The vessels were allowed to remain on ice for approximately one hour. Following the vessels were air dried and a final mass was recorded and compared to the initial mass to ensure than the total difference in initial and final sample mass was $\leq 20 \mathrm{mg}$. The samples were then diluted with $1 \mathrm{~mL}$ of Milli-Q boron-free water and split $1.5 \mathrm{~mL}$ for boron isotope ratio analysis and $1.5 \mathrm{~mL}$ for lanthanide series rare earth element (REE) analysis, which is described in the following subchapter.

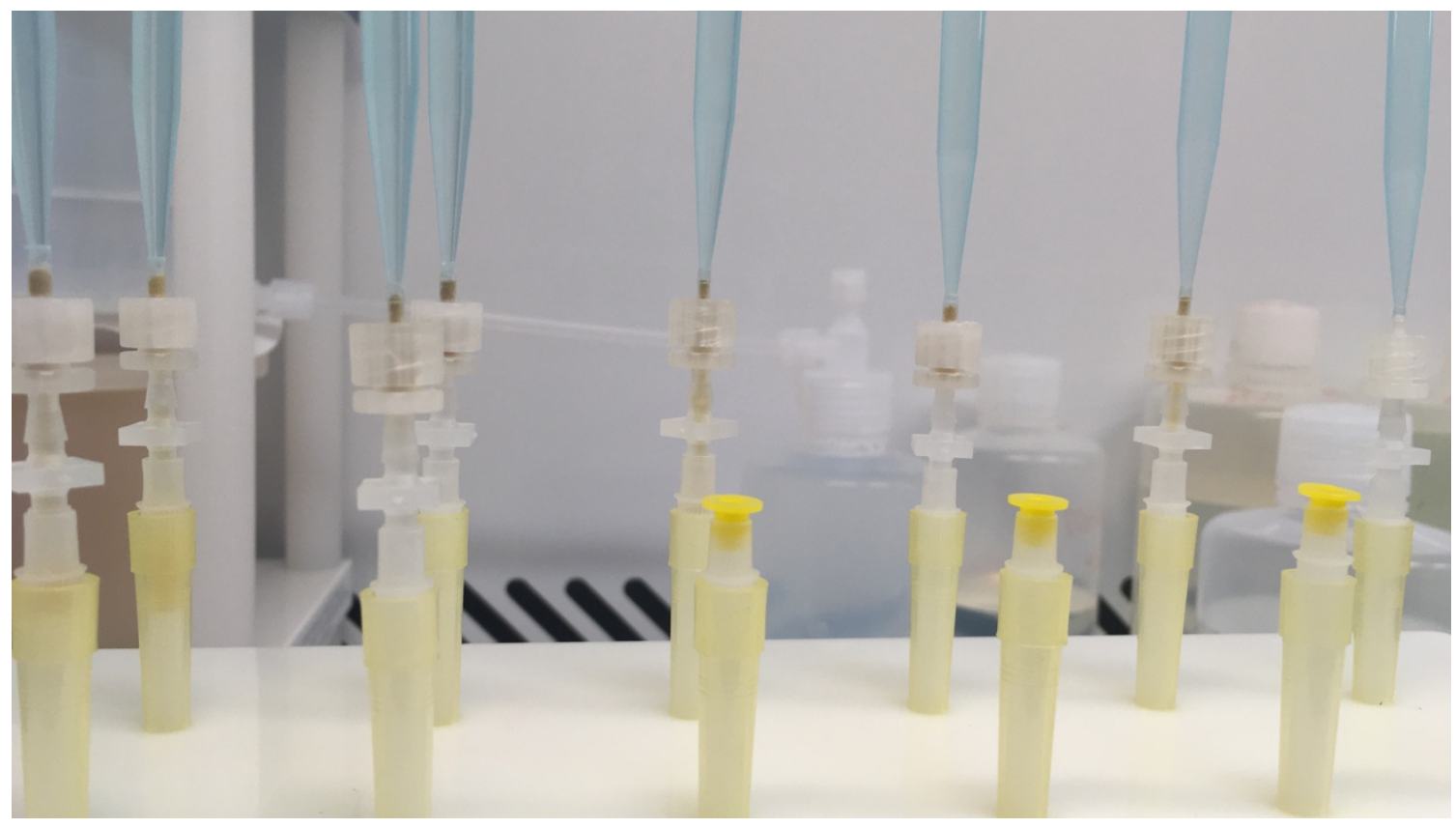

Figure 34. Luer lock assembly of boron exchange separation columns, packed with ground Amberlite IRA743 resin and filtered using Whatman ashless filter paper. 
The separation of boron from other matrix elements was accomplished by column chromatography using Amberlite resin as a stationary phase. In slightly basic conditions at a $\mathrm{pH}$ of 8 , the resin has a greater affinity for the resin solid phase compared to the liquid sample. Therefore, a basic titration with Optima grade ammonium hydroxide, $\mathrm{NH}_{4} \mathrm{OH}$, was performed following on the fraction of sample reserved for boron isotope analysis. The solutions were titrated to approximately a $\mathrm{pH}$ of $8.5-9$ by regularly testing on multicolored $\mathrm{pH}$ paper. However, the heroin samples changed from yellow to orange in this $\mathrm{pH}$ range which made the titrations much easier. Once the samples had been titrated to a basic $\mathrm{pH}$, they were centrifuged for $30 \mathrm{~s}$ at $4000 \mathrm{rpm}$.

Boron ion exchange columns were prepared similarly to the method described in Chapter 3 with luer lock microcolumns (see Figure 34), but for boron analysis the solid phase exchange media was Amberlite IRA-743, which was manually ground with a mortar and pestle and mesh filtered to utilize only resin particles with diameters between 50 - 100 $\mu \mathrm{M}$. The reason the beads were ground was to ensure the packing of the resin was dense and the flow rates through the columns was more consistent, and the resin surface area was maximized. It has been reported that column exchange separation with Amberlite IRA-743 can cause fractionation in boron isotopes, but during optimization it was found that grinding the resin also mitigated the on-column boron isotope fractionation.

\subsubsection{Results}

The results of these experiments proved to be unsuccessful at producing reproducible ratios for even duplicate preparations of the same samples. This is likely in minor part from degassing upon addition of the $1 \mathrm{~mL}$ Milli-Q water but primarily during 
titration of the sample with $\mathrm{NH}_{4} \mathrm{OH}$ to a $\mathrm{pH}$ which produced significant gases. Volatilization results in fractionation of ${ }^{10} \mathrm{~B}$ and ${ }^{11} \mathrm{~B}$, with the lighter mass isotope favoring the gas phase over the solution. Fractionation to even a slight degree produces large variations in the per mille $\%$ calculation of $\delta{ }^{11} \mathrm{~B}$ because of the relatively large difference in atomic mass of the two isotopes.

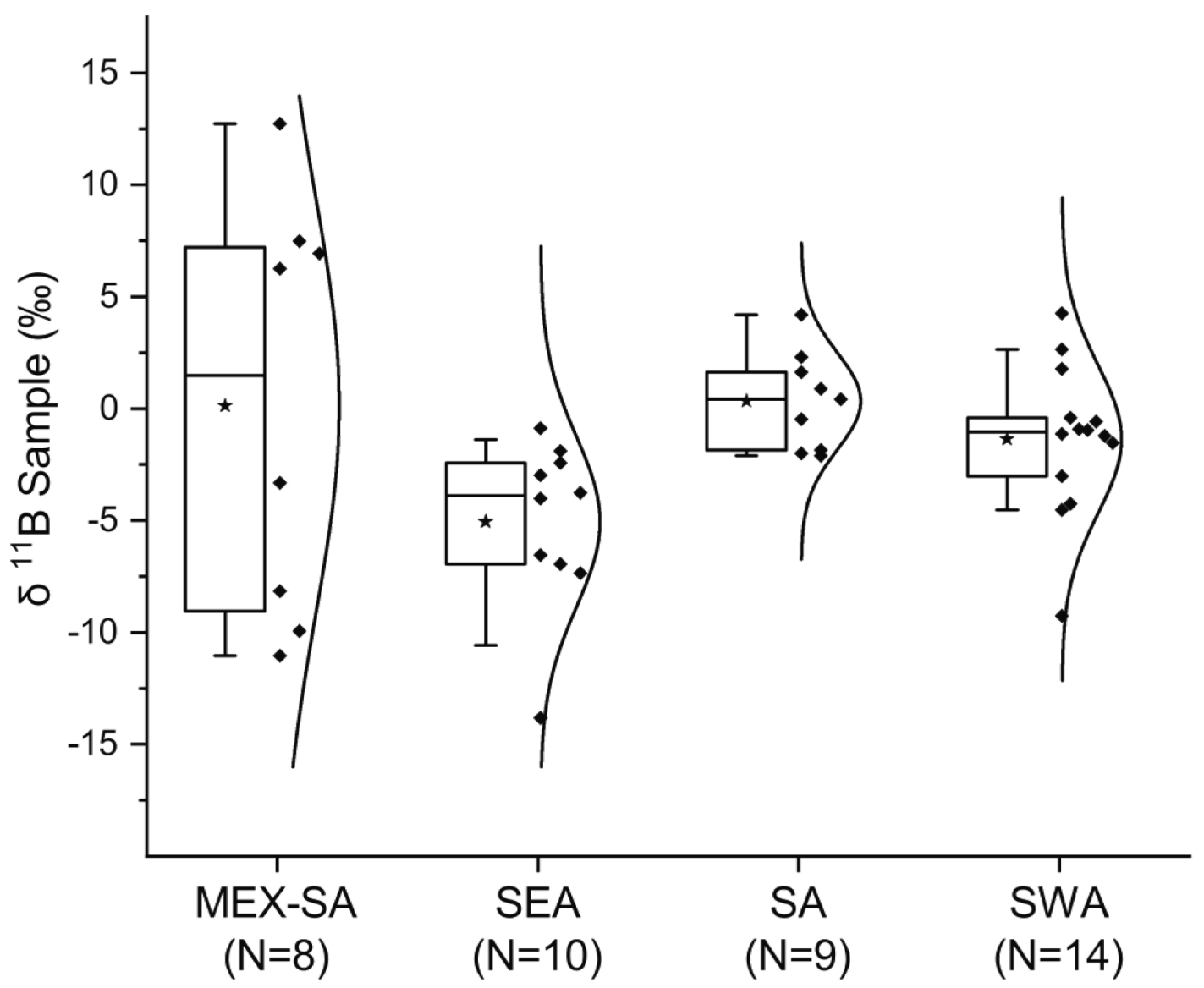

Figure 35. Box plot and data points of boron isotope ratio $\left(\delta^{11} \mathrm{~B}\right)$ measured in heroin samples by region of production. Box plot shows $25 \%$ to $75 \%$ quantile within the boxes and $5 \%$ to $95 \%$ within the whiskers. The median value of each region is indicated by the horizontal line within the boxes and the mean value of the region by the star.

As shown on Figure 35, there is a great deal of overlap in the data point of each of the four regions. While the data set of each group is limited, the ranges of SEA, SA and 
SWA appear to be much tighter than that of MEX-SA. There are no data points representative of the mean and median value of MEX-SA, suggesting that the distribution of data in this region may not be normally distributed. As shown on Table 22, the comparison of data to the group means is not successful in profiling the samples and nearly $60 \%$ of data is assigned to an incorrect origin.

Table 22. Prediction of Origin by means comparison of boron $\delta 10 / 11$ values by region

\begin{tabular}{|c|c|c|c|c|c|}
\hline \multirow[b]{2}{*}{ Actual Origin } & \multicolumn{5}{|c|}{ Predicted Count for Training Set } \\
\hline & MEX-SA & SA & SEA & SWA & $\%$ Correct \\
\hline MEX-SA $(n=8)$ & 0 & 4 & 4 & 0 & $0 \%$ \\
\hline $\mathrm{SA}(\mathrm{n}=9)$ & 1 & 5 & 0 & 3 & $56 \%$ \\
\hline $\operatorname{SEA}(n=10)$ & 0 & 0 & 6 & 4 & $60 \%$ \\
\hline SWA $(n=14)$ & 2 & 3 & 3 & 6 & $43 \%$ \\
\hline $\operatorname{SUM}(n=41)$ & 3 & 12 & 13 & 13 & $41 \%$ \\
\hline$\%$ Confusion & $100 \%$ & $58 \%$ & $54 \%$ & $54 \%$ & \\
\hline
\end{tabular}

\section{2 $\mathrm{Pb}$ Isotope Ratios}

Lead isotope analysis was also proposed as a potential system, however only $10 \%$ of all samples met concentrations similar to the specified concentrations set for strontium isotope analysis, which allowed for approximately $50 \%$ of the samples $(n=195)$ to be analyzed. The median concentration of $\mathrm{Pb}$ was $0.269 \mu \mathrm{g} / \mathrm{g}$, whereas the median $\mathrm{Sr}$ concentration was $2.3 \mu \mathrm{g} / \mathrm{g}$. Table 23 shows that $90 \%$ of all samples contained $<1.7 \mu \mathrm{g} / \mathrm{g}$ of $\mathrm{Pb}$. Given that the average dilution of samples was $\sim 350 \mathrm{x}$ that would make the frequency of samples with more than $5 \mathrm{ppb}$ in the diluted solutions $\leq 10 \%$ of the total. Because a 
solution of $>5 \mathrm{ppb}$ was desired for precise isotope ratio measurement, analysis of $\mathrm{Pb}$ isotope ratios was not explored further in this research.

Table 23: Quantiles of distribution of total $\mathrm{Pb}$ concentrations $(\mu \mathrm{g} / \mathrm{g})$.

\begin{tabular}{|l|c|r|}
\hline $100.00 \%$ & maximum & 255.269 \\
\hline $99.50 \%$ & & 212.501 \\
\hline $97.50 \%$ & & 13.887 \\
\hline $90.00 \%$ & & 1.689 \\
\hline $75.00 \%$ & quartile & 0.733 \\
\hline $50.00 \%$ & median & 0.269 \\
\hline $25.00 \%$ & quartile & 0.099 \\
\hline $10.00 \%$ & & 0.033 \\
\hline $2.50 \%$ & & 0.006 \\
\hline $0.50 \%$ & & 0.002 \\
\hline $0.00 \%$ & minimum & 0.002 \\
\hline
\end{tabular}

\subsection{Assessment of Adulterant Effects}

One of the concerns of the research project into provenance determination of heroin samples, was regarding the applicability of the method to street-level samples, which are typically moderately to heavily adulterated with diluent materials. Caffeine, dextromethorphan and lidocaine are commonly occurring small organic molecules with CNS activity which can be used to cut heroin samples and increase the profit of street-level dealers by increasing the mass of their product with cheap compounds. However, depending upon the appearance of the heroin, which can be extremely varied, it may be impossible to predict what a dealer might use to dilute their sample. 

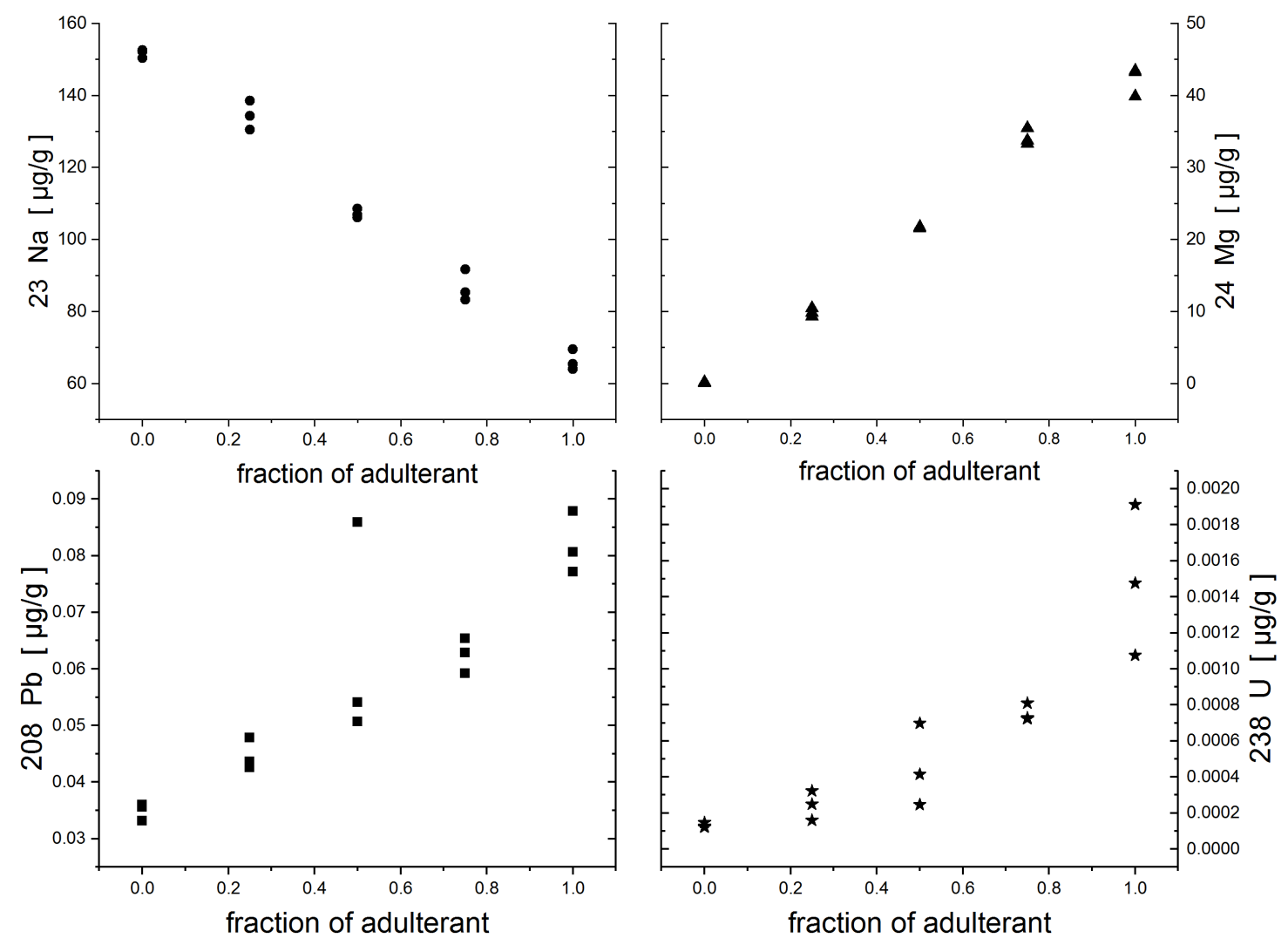

Figure 36. $\mathrm{X} / \mathrm{Y}$ plot of measured elemental concentrations ${ }^{23} \mathrm{Na},{ }^{24} \mathrm{Mg},{ }^{208} \mathrm{~Pb}$ and ${ }^{238} \mathrm{U}$ over the fraction of adulterant, 03402 - caffeine, in a mixture with a test heroin sample. The neat heroin sample is at the 0 fraction and the neat cut is at the 1.0 fraction.

To assess the extent to which an elemental profile of a heroin sample might be affected by a diluent, a SA heroin sample was cut with 3 authentic seized adulterant compounds at varying ratios of heroin to adulterant. The first cutting agent (03402) was caffeine; the second (03539) was a mixture of caffeine and acetaminophen; and the third (89323) was a mixture of caffeine, acetaminophen and dextromethorphan. Table 24 shows the effect of the cutting material on elements relevant to provenance determination, which are given in Chapter 2. A negative slope indicates that increase cutting material will shift the elemental concentration of the bulk sample lower, while a positive slope indicates that 
the cutting material will add to the elemental concentration of the bulk sample. The plots shown on Figure 36 show the results for the mixture of the 03402 caffeine sample with the test heroin sample.

Table 24. Pearson correlation coefficients and linear relationship slope shown for all elements with $\mathrm{R}^{2}>0.7$

\begin{tabular}{|c|c|c|c|c|c|c|}
\hline \multirow[b]{2}{*}{ Element } & \multicolumn{2}{|c|}{03402} & \multicolumn{2}{|c|}{03539} & \multicolumn{2}{|c|}{89323} \\
\hline & $\mathrm{R}^{2}$ & Slope & $\mathrm{R}^{2}$ & Slope & $\mathrm{R}^{2}$ & Slope \\
\hline${ }^{23} \mathrm{Na}$ & 0.9899 & -87.4 & 0.9955 & -116 & 0.8252 & 26.3 \\
\hline${ }^{24} \mathrm{Mg}$ & 0.9926 & 43.3 & & & 0.9949 & 147 \\
\hline${ }^{52} \mathrm{Cr}$ & & & 0.0986 & -0.682 & & \\
\hline${ }^{57} \mathrm{Fe}$ & & & 0.7534 & 57.8 & 0.9731 & 145 \\
\hline${ }^{66} \mathrm{Zn}$ & & & & & 0.8246 & 4.51 \\
\hline${ }^{90} \mathrm{Zr}$ & & & & & & \\
\hline${ }^{111} \mathrm{Cd}$ & & & & & & \\
\hline${ }^{208} \mathrm{~Pb}$ & 0.7734 & 0.045 & & & & \\
\hline${ }^{238} \mathrm{U}$ & 0.7772 & 0.0013 & 0.9936 & 0.024 & 0.9975 & 0.023 \\
\hline
\end{tabular}

\subsection{Analysis of Volatile and Semi-Volatile Organic Compounds}

\subsubsection{Abstract}

A sampling method to chemically analyze the enclosed headspace of heroin was investigated for its utility in capturing and subsequently liberating volatile and semivolatile organic compounds for identification by gas chromatography/mass spectrometry (GC/MS). A packed capillary tube designed to capture compounds from headspace, known as capillary micro-extraction of volatiles (CMV), was set up to sample the headspace above heroin samples of approximately $50 \mathrm{mg}$ in total mass. As a preliminary examination of this application, one heroin sample from each of the four major heroin-producing regions (Mexico, South America, Southeast Asia and Southwest Asia) was sampled by this method. Several of the detected compounds were identified with the use of chemical reference 
standards. Some compounds, such as acetic acid and methylacetamide, were found in samples from each of the four regions; these compounds may have the potential to be used for heroin screening of seized drugs. On the other hand, compounds that were unique to one region may be used instead for chemical profiling strategies and origin prediction for the purpose of gathering strategic intelligence and combatting the illegal drug trade.

\subsubsection{Introduction}

The CMV device was developed from the principles of solid phase micro-extraction (SPME) wherein a fiber coated with polydimethylsiloxane (PDMS) is allowed to passively absorb volatile organic compounds (VOCs) from the headspace volume in a closed contained $[88,89]$. A PDMS-coated glass filter is cut into uniform strips and packed inside of a $2 \mathrm{~cm}$ open-ended capillary tube with an inner diameter of $2 \mathrm{~mm}$ [89-91]. The openended configuration enables dynamic air sampling via attachment of a vacuum pump. Chemical modifications to the sol-gel formula (Phenyl-PDMS versus the original PDMS) enabled tuning of the CMV device for improved sampling efficiency of more volatile organic compounds; the formula described was utilized in this application for sampling heroin headspace VOCs [92].

The analysis of headspace compounds by CMV-GC-MS has been used to sample forensic samples such as explosives resides, gunshot residues and controlled substances [90, 93-95]. Additionally, Nair and Miskelly used capillary microextraction devices for sampling methamphetamine vapor [96]. The analysis of VOCs by CMV is a dynamic headspace sampling method; therefore, there is no direct sample interaction and negligible loss of mass to the heroin samples. The ability to detect the VOCs in heroin samples can 
permit rapid screening and the non-destructive nature of the method facilitates follow-up confirmatory techniques using the same sample aliquot.

The four samples were selected for having substantial quantity for replicate testing and high-purity, between $64 \%$ and $74 \%$ heroin content by mass. They are referred to as MEX, SA, SEA and SWA (Mexico, South America, Southeast Asia and Southwest Asia) for the sake of discretion and simplicity. The SWA sample had unknown heroin purity, however it was used as the duplicate control standard for research related to the heroin's inorganic analysis [62], so it was selected for the analysis by this method as well. However, as described in the results, the Southwest Asian sample appeared to suffer from chemical changes related to the heat, which was not observed in the other samples.

\subsubsection{Methods and Materials}

A set of three CMV devices were prepared and assembled in the laboratory according to the protocol described previously [90,91]. All analyses were performed using the previously described CMV-A formulation [92]. A sand bath was heated with a GlasCol PL 100D heater with a 104A PL612K Digitrol II digital temperature control with thermocouple input (Glas-Col, Terre Haute, IN). Approximately $50 \mathrm{mg}$ of the heroin samples were placed inside $15 \mathrm{~mL}$ glass headspace vials with silicone/PFTE septa (Supelco, Bellefonte, PA). Air sampling was performed with a Bailey Nurture III pump connected to a flow meter using Tygon tubing. The CMV device was connected to the pump via Silastic and PFA tubing. A 16-gauge needle was securely connected to the tubing in an airtight manner to pierce through the septa and facilitate headspace sampling. 


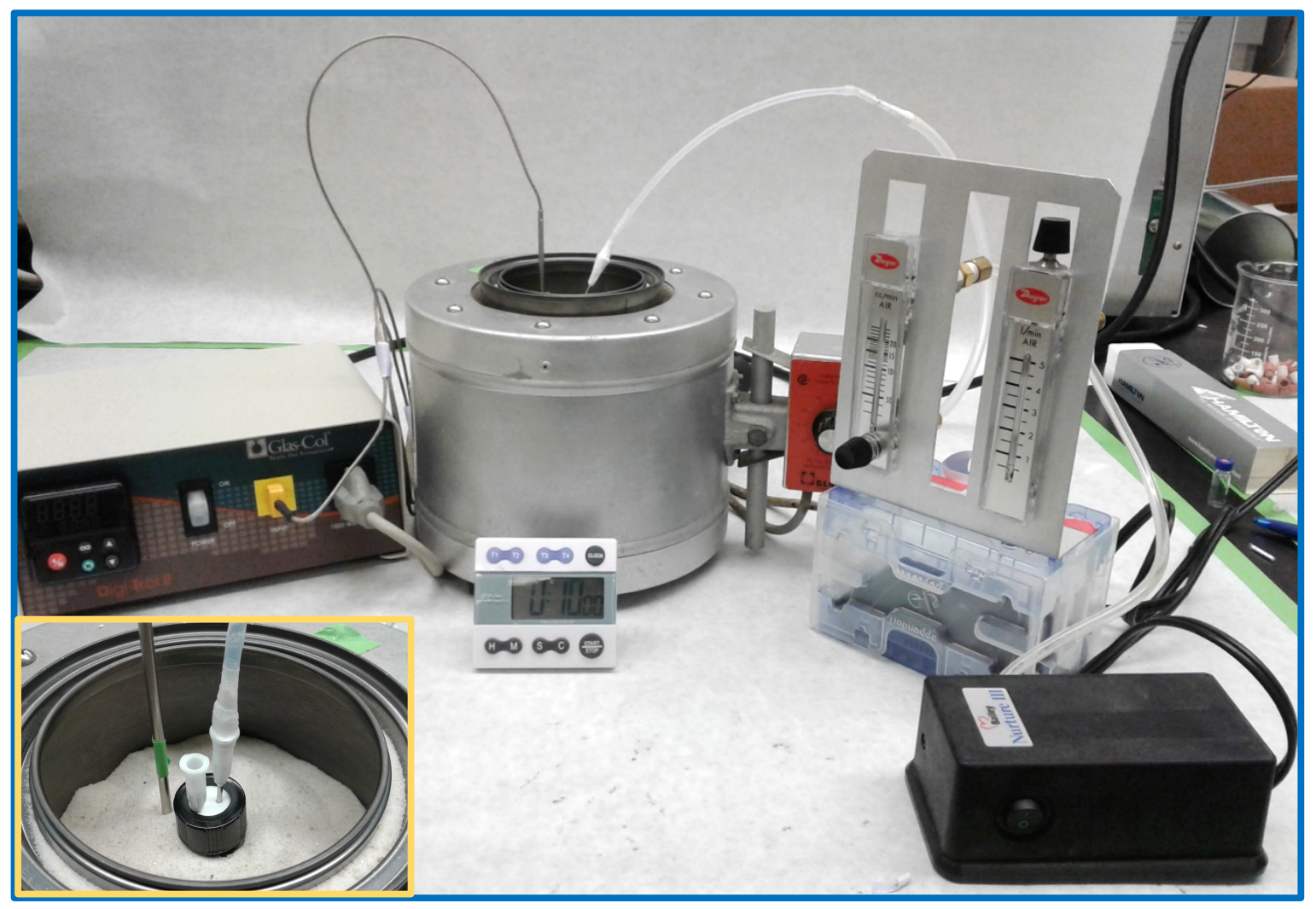

Figure 37. Sampling setup for headspace extraction of heroin samples using CMV.

First, CMVs were preconditioned in an oven at $250^{\circ} \mathrm{C}$ and desorbed in the GC inlet as a blank. Authentic heroin samples were weighed $(50 \mathrm{mg})$ into headspace vials, sealed, and immersed in a sand bath at $150^{\circ} \mathrm{C}$ for 10 minutes of equilibrium time. The CMV was connected via airtight tubing to the air sampling pump as depicted in Figure 37. Next, the pump was turned on while piercing the septum of the vial with the needle to begin sampling. A second needle was inserted to allow ambient air entry to prevent a vacuum buildup. Sampling was performed at $0.2 \mathrm{~L} / \mathrm{min}$ for 10 minutes for a total of $2 \mathrm{~L}$ of air sampled. These parameters were selected on the basis of the results of previous experiments using the CMV for extracting volatiles in ambient air $[92,97]$. Immediately after pumping, the CMVs were placed in the thermal desorption probe and inserted into 
the GC inlet for analysis. Three replicate analyses of each type of heroin were performed using this technique. Blanks of the entire setup were also analyzed using empty vials in an identical manner to the actual samples.

An Agilent 7890A gas chromatograph connected to a 5975C inert XL mass spectrometer with a triple axis detector (Agilent Technologies, Santa Clara, CA) was used for sample analysis. The CMV devices were thermally desorbed by placing them on an Agilent Thermal Separation Probe installed on the split/splitless GC injection port. A VF624 column (30 m x $0.25 \mathrm{~mm} \times 1.40 \mu \mathrm{m}$, Agilent Technologies, Santa Clara, CA) was used for chromatographic separation with a helium flow rate of $1.2 \mathrm{~mL} / \mathrm{min}$. A Sky ${ }^{\circledR} \mathrm{mm}$ ID single taper inlet liner (Restek, Bellefonte, PA) was used and the inlet was set at $150{ }^{\circ} \mathrm{C}$ in a split mode at 5:1 ratio. The oven temperature program of the $\mathrm{GC}$ began at $35{ }^{\circ} \mathrm{C}$ and held for $5 \mathrm{~min}$, followed by an increase to $50{ }^{\circ} \mathrm{C}$ at $10{ }^{\circ} \mathrm{C} / \mathrm{min}$ for $1 \mathrm{~min}$, to $80{ }^{\circ} \mathrm{C}$ at $30{ }^{\circ} \mathrm{C} / \mathrm{min}$, then $150^{\circ} \mathrm{C}$ at a rate of $10^{\circ} \mathrm{C} / \mathrm{min}$, to $240{ }^{\circ} \mathrm{C}$ at a rate of $20^{\circ} \mathrm{C} / \mathrm{min}$, and finally to $270{ }^{\circ} \mathrm{C}$ at $30{ }^{\circ} \mathrm{C} / \mathrm{min}$ for $1 \mathrm{~min}(22 \mathrm{~min}$ run time). The temperatures of the EI source, the transfer line to the mass spectrometer, and the quadrupoles were set to $230{ }^{\circ} \mathrm{C}, 280{ }^{\circ} \mathrm{C}$, and $150{ }^{\circ} \mathrm{C}$, respectively. The mass scan range was set at 33-300 amu. The resolution of the mass analyzer was $0.1 \mathrm{amu}$. The instrument was tuned before the experiments using the autotune feature as recommended by the manufacturer.

\subsubsection{Results}

The resulting chromatograms from each CMV analysis were processed using the accompanying Agilent ChemStation (version E.02.01.1177) software. Method blanks were overlaid with sample chromatograms to locate peaks of interest and their retention times. 
Next, preliminary compound identification of these peaks was performed by searching background subtracted mass spectra with the NIST 08 Mass Spectral Library. Standard solutions of several of these compounds were prepared at either 50 or $100 \mathrm{ppm}$ in HPLC grade methanol. A direct spike of $1 \mu \mathrm{L}$ of each solution was spiked on the CMV one at a time and analyzed with the same GC-MS method. The mass spectra and retention times of the standard solutions were compared with the compounds previously found in the samples to confirm their presence.

Table 25: List of compounds identified in headspace of four unique heroin samples using CMV$\mathrm{GC} / \mathrm{MS}$ technique.

Ranked \#1-10 by Integrated Area $(\mathrm{n}=3) \#=$ minor $(<$ rank 10$) ; *=$ NOT confirmed Heroin Sample \# $(\mathrm{n}=3)$

\begin{tabular}{|c|c|c|c|c|c|}
\hline R.T. (min) & Compound Name & SA & SEA & MEX & SWA \\
\hline 4.859 & Diethyl ether* & & 8 & & \\
\hline 5.438 & Acetone & & & 9 & \\
\hline 8.567 & $\begin{array}{l}\text { Ethyl methyl ketone (2- } \\
\text { butanone)* }\end{array}$ & 4 & & & \\
\hline 8.626 & Ethyl acetate & & 3 & 1 & \\
\hline 9.803 & Acetic acid & 1 & 1 & 2 & 1 \\
\hline 13.351 & Butanoic acid & \# & & & \\
\hline 13.780 & Acetamide & 3 & 4 & 5 & 3 \\
\hline 14.488 & Isovaleric acid* & & & & 7 \\
\hline 14.598 & 2-Methylbutanoic acid* & & & & 9 \\
\hline 14.771 & Methylacetamide & 2 & 2 & 7 & 2 \\
\hline 16.584 & Hexanoic Acid & 8 & 7 & 4 & \# \\
\hline 17.013 & Ethylhexanol & & & 3 & \\
\hline 17.252 & Phenol & & 5 & 6 & 8 \\
\hline 18.225 & $\begin{array}{l}\text { 2-Ethyl-1-hexanol } \\
\text { acetate }\end{array}$ & & & 10 & \\
\hline 18.304 & p-cresol & 7 & 10 & & 10 \\
\hline 18.844 & Octanoic acid* & & 9 & & \\
\hline 19.972 & p-anisaldehyde & 6 & & & 4 \\
\hline 20.321 & Triacetin* & 9 & & & \\
\hline 20.870 & Longifolene & & & & 6 \\
\hline 21.417 & Meconin* & 5 & 6 & 8 & \\
\hline 21.512 & Veratraldehyde & 10 & & & 5 \\
\hline
\end{tabular}


A list of compounds identified in the four heroin samples is presented in Table 25. The average integrated area $(n=3)$ underneath each peak was obtained and ranked from 1 (greatest area) to 10 (lower area) for each compound present in each sample. Acetic acid was present in all four samples with very large peak height and integrated area. Acetamide, methylacetamide, and hexanoic acid were also present in all four samples.

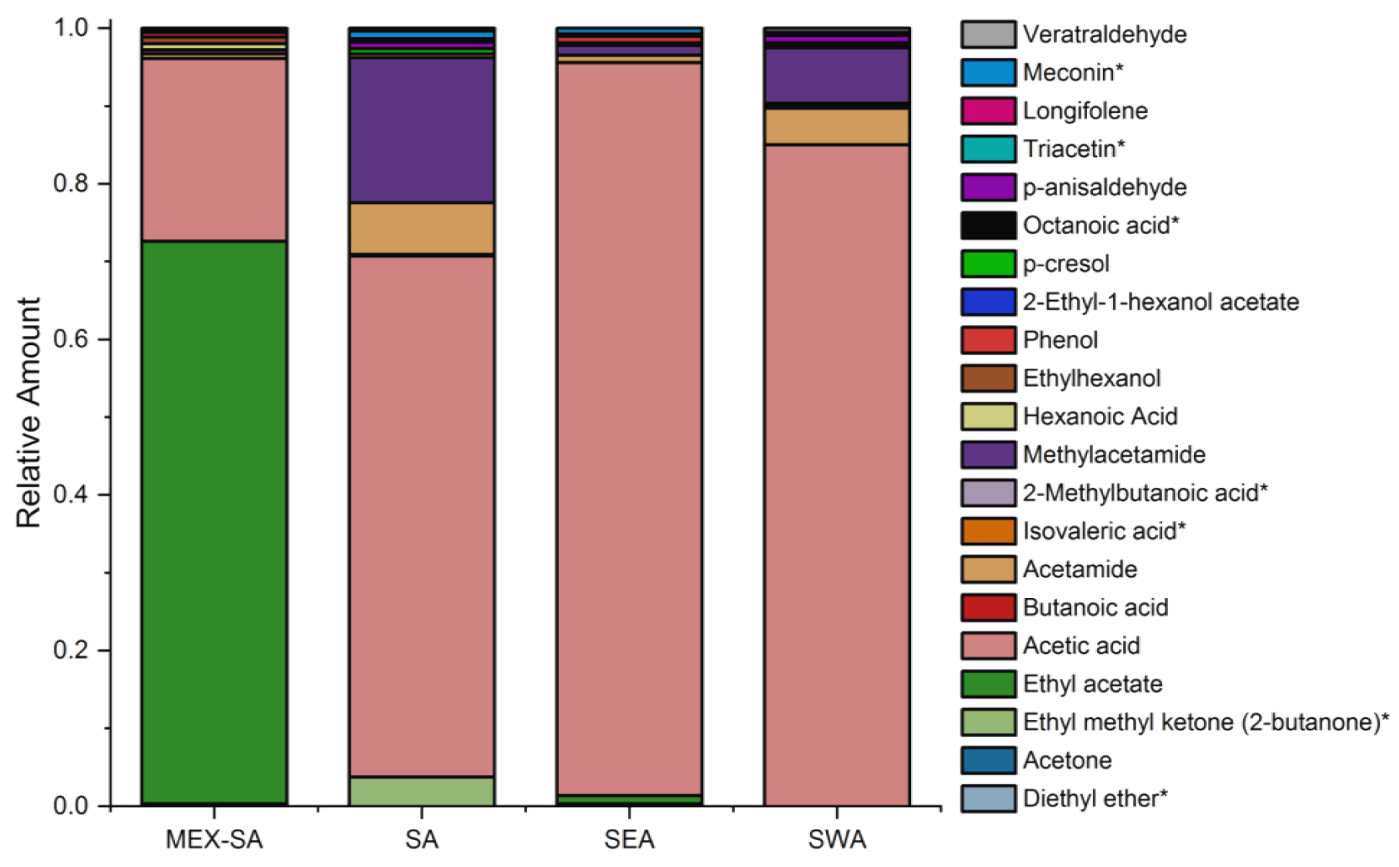

Figure 38: Relative abundance of organic compounds in the headspace of heroin samples by region

As shown in Figure 38 and Figure 39, the combined relative abundance of the first and second peaks is significantly larger than for the remaining peaks in the chromatograms. Apart from the MEX-SA sample, the primary compound is acetic acid. The very large peak for ethyl acetate within the MEX-SA ( $\sim 72 \%$ relative abundance) sample may give some means of differentiation between MEX-SA heroin samples and those from the other three regions; ethyl acetate was only otherwise detected in the SEA sample at a relative 
abundance of $\sim 1 \%$. Ethyl methyl ketone was uniquely detected in the SA sample. The SWA samples, which were visibly blackened upon heating, yielded chromatograms with the most peaks, which included several unidentified peaks. A notable compound detected in the SWA sample, longifolene, is found in the resin of a pine species that is native to the region [98]. Meconin, a constituent of the opium poppy, was detected in three samples and has been previously detected in GC-MS studies of illicit heroin [99].

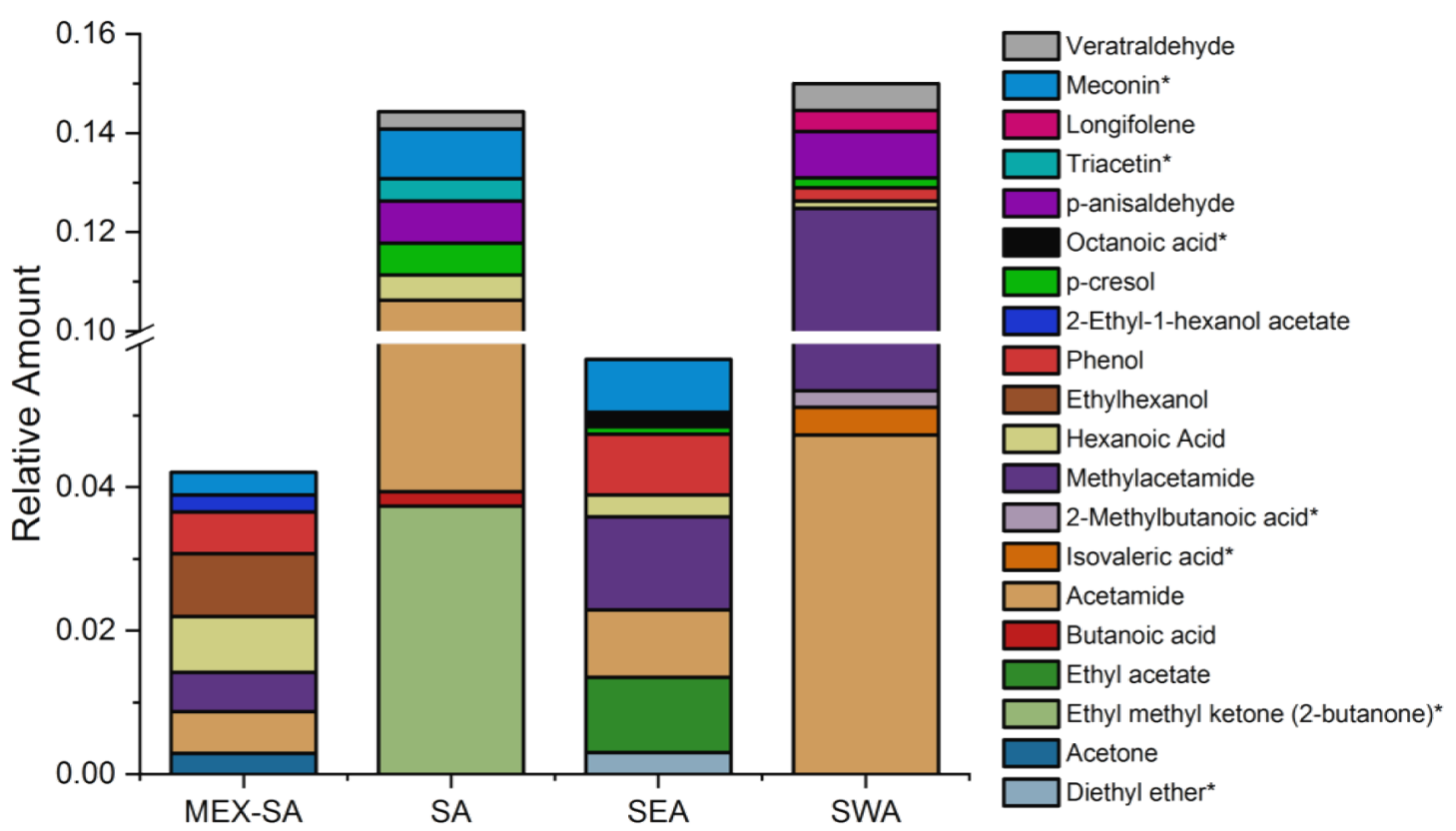

Figure 39: Relative abundance of organic compounds in the headspace of heroin samples by region, compounds $\geq 15 \%$ relative abundance not shown.

\subsection{Conclusions}

The results of the boron isotope study were inconclusive as there were observed difficulties with both sample preparation and analysis. A more robust method for sample preparation may have greater success at evaluating the potential of the $\delta^{11} \mathrm{~B}$ systematic for 
provenance determination of heroin samples. However, the chemistry of boron isotopes makes the expectation of boron fractionation during illicit processing a near certainty. Therefore, the observed $\delta^{11} \mathrm{~B}$ value would be more characteristic of the regional processing methodology rather than characteristic of the environment in which the opium poppies are grown, harvested and processed into heroin.

The quantitative analysis of lead precluded its isotopic analysis on the basis of the infrequency at which sufficient lead abundances were found in heroin samples. This is especially the case because the ${ }^{208} \mathrm{~Pb}[\mu \mathrm{g} / \mathrm{g}]$ was actually calculated from measured ${ }^{206+207+208} \mathrm{~Pb}[\mu \mathrm{g} / \mathrm{g}]$. Using the same criterion for analysis as was made for ${ }^{87} \mathrm{Sr} /{ }^{86} \mathrm{Sr}$ in Chapter 4 , fewer than $10 \%$ of samples would have had adequate lead concentrations for precise measurement of $\mathrm{Pb}$ isotope ratios by MC-ICP-MS. Perhaps digestion of a larger mass of sample and minimal post-digestion dilution may permit $\mathrm{Pb}$ isotope measurements in future studies.

The analysis of elemental concentration of the authentic cutting materials showed that the elemental profile of several, key elements were significantly changed by the cutting agent even at a ratio of 4:1 heroin to cutting material. In some elements, sodium for example, the cutting agents may contain less than the test sample of heroin and reduced the measured mass concentration of the element when adulterated. However, in general, the cutting agents had greater concentrations for elements such as $\mathrm{Mg}, \mathrm{Fe}$ and $\mathrm{U}$ than were found in the test sample of heroin. Generalizations from such a small experiment using a single sample of heroin should not be made hastily, but precautions should be made when attempting provenance determination using trace elements to avoid adulterated samples as 
much as possible and to consider the effect of adulterant present. Forensic sample matching, on the other hand, likely would not be affected as the majority of the samples entered as evidence would either both be uncut, bulk samples or adulterated, street-level samples.

The results of the headspace sampling experiments demonstrate the potential for analysis of volatile and semi-volatile organic compounds for profiling heroin samples via CMV-GC-MS. A variety of semi-volatile as well as volatile organic compounds were detected in the headspace, despite initially searching for residual solvents and VOCs that were identified by other researchers, some of which could be targets for heroin profiling as they were present in only one regional sample or in greatly different proportions between samples. The CMV-GC-MS method utilized in this study is a fast and non-destructive analysis technique with the potential for identifying volatile signatures from heroin as well as other illicit drugs of interest. A much larger-scale analysis of heroin samples by CMVGC-MS is recommended given the observations, to determine if there are trends among heroin of common origin with respect to VOCs in their headspace. 


\section{CHAPTER 6. CONCLUSIONS}

In this dissertation is reported the method details of the first known large-scale provenance determination study of heroin on the basis of inorganic analysis, wherein authentic samples representing all four of major heroin producing regions are analyzed by ICP-MS. Using multivariate discriminant analysis of quantitative data for minor and trace elements, correct provenance was determined for $88 \%$ of samples in a test sample set. The modelling of data by linear discriminant analysis was made through carefully selecting the most informative elements and removal of highly correlated elements such as $\mathrm{Ca} / \mathrm{Sr}$ and $\mathrm{Zr} / \mathrm{Hf}$. The most informative and uncorrelated elements which were included in the model were ${ }^{23} \mathrm{Na},{ }^{24} \mathrm{Mg},{ }^{52} \mathrm{Cr},{ }^{57} \mathrm{Fe},{ }^{66} \mathrm{Zn},{ }^{90} \mathrm{Zr},{ }^{111} \mathrm{Cd},{ }^{208} \mathrm{~Pb}$ and ${ }^{238} \mathrm{U}$. Each element helped to differentiate and associate heroin samples in one of three ways, either by showing differences in the distribution of data between heroin from Eastern (SEA/SWA) and Western Hemispheres (MEX-SA/SA), differences between SEA and SWA or differences between MEX-SA and SA.

The results suggest that using a relatively affordable instrument, Q-ICP-MS, with highly efficient and reproducible sample preparation can determine provenance for $88 \%$ of heroin samples, which was a significant observation as many research labs do not have the budget to invest in HR-ICP-MS instrumentation. The use of HR-ICP-MS provided no clear advantages over the Q-ICP-MS because of the analytical challenges experienced, which were inaccurate recovery calculations and high inter-day relative standard deviations for several elements in the standard reference material and control heroin sample. 
The data from the Q-ICP-MS also proved to be useful in sample-to-sample comparisons for seizure association purposes. Rates of false inclusion and false exclusion as well as evaluation of the sample comparison method performance was also evaluated using the quantitative data obtained in Chapter 2. The rate of type 1 errors, or false exclusions, was found to be $4.17 \%$ and the rate of type 2 errors, or false inclusions, was found to be $0.26 \%$ when using a match criterion of $\pm 3 s$. Because the availability of background information related to the production and trafficking histories is limited even for authentic heroin samples, few assumptions about correct sample association can be made. However, many of the samples believed to be related were found to be indistinguishable based upon their elemental profiles.

In addition, the manuscript comprising Chapter 4 of this dissertation is the first reporting of ${ }^{87} \mathrm{Sr} /{ }^{86} \mathrm{Sr}$ isotope ratio analysis of heroin and proves the usefulness of the radiogenic strontium systematic for the purpose of predicting geographic origin of heroin [62]. It was important to have a characterization of elemental concentration of samples, prior to an attempt at $\mathrm{Sr}$ (or other) isotopic ratio analysis. The results of the MC-ICP-MS strontium ratio analysis of ${ }^{87} \mathrm{Sr} /{ }^{86} \mathrm{Sr}$ have been demonstrated to perform at $80 \%$ or better correct classification rate of blind heroin samples when focusing on samples of only MEXSA and SA origins and only using the initial small training set of heroin samples. Adding the other two main producers (SEA and SWA) decreases the correct prediction rate of blind samples to $75 \%$.

The method for sample preparation, elemental instrumental analysis, column chemistry and isotope instrumental analysis were described in relevant chapters. The 
methods were validated using reagent and method blanks, standard reference materials and duplicate matrix control samples at every step of method development and sample processing.

Combining 6 elemental quantities with strontium isotopic data had similar performance as was observed in the 9 best elemental quantities (88\%), but because the origins of the blind test samples are known to the DEA-STRL, these samples can be incorporated into a new, larger training set (database) that the DEA-STRL can use going forward. Combining all samples for which strontium data was available with the elemental data of those samples, produced correct associations for $93 \%$ of the samples. Since the number of samples in the database increased significantly over the testing set used here, model performance was shown to improve. The performance of the model using the complete data set was validated using leave-one-out cross-validation.

Recommendations from this research for DEA-STRL scientists and other forensic drug chemists/analysts are to incorporate the validated methods of microwave-assisted digestion for the preparation of heroin samples and inorganic quantitative method via QICP-MS. Using the elemental data in conjunction with other signature techniques already used in casework are expected to help with any ambiguities in assignment of origin. On their own, minor and trace elemental profiles are estimated to be able to correctly assign provenance to $88 \%$ of unknown samples. In addition, minor and trace elemental profiles can associate heroin samples in forensic sample comparisons better than $95 \%$ of the time.

Any laboratories attempting to use these methods should maintain rigorous use of reference standards and control standards, as well as review performance data daily to 
verify the robustness of the method with different operators and under different maintenance conditions. Preparing samples via Sr affinity resin by this method is relatively easy and inexpensive and results in samples of $0.01 \mathrm{M} \mathrm{HNO}_{3}$ that can be safely be shipped to an external laboratory for ${ }^{87} \mathrm{Sr} /{ }^{86} \mathrm{Sr}$ determination if an in-house instrumental facility were not available.

The results of the analysis of volatiles and semi-volatiles by CMV-GC/MS suggests that the method may have potential for provenance determination if investigated on a larger scale. There were unique compounds identified in each regional sample that may allow for heroin samples to be profiled based upon their presence and/or relative abundances. The method provides a means to sample headspace without sample destruction, however it must be performed at $150^{\circ} \mathrm{C}$ which can occasionally produce chemical changes within the sample matrix. A more comprehensive evaluation of the CMV-GC/MS technique applied to authentic heroin samples may produce a novel method for profiling heroin samples.

The research described in this dissertation has produced methods for highthroughput sample preparation and analysis of minor and trace elemental impurities of illicit powder heroin, which are amenable for conducting provenance determination studies for gathering strategic intelligence on heroin as well as for making forensic sample comparisons of heroin evidence for criminal investigations. The research also takes heroin research to new frontiers with radiogenic strontium isotope ratio analysis for provenance determination and may inspire future research into $\left({ }^{87} \mathrm{Sr} /{ }^{86} \mathrm{Sr}\right)$ analysis of other illicit drug matrices. Lastly the research opens the door for CMV-GC/MS as a new technique to be evaluated for use in the arsenal of drug chemists working to curtail the heroin epidemic. 


\section{REFERENCES}

1. Wright, C.R.A., On the action of organic acids and their anhydrides on the natural alkaloids. Part I. Journal of the Chemical Society, 1874. 27: p. 1031-1043.

2. Sneader, W., The discovery of heroin. Lancet, 1998. 352(9141): p. 1697-1699.

3. United Nations International Drug Control Programme. Laboratory Section., Recommended Methods for Testing Opium, Morphine and Heroin: Manual for Use by National Drug Testing Laboratories. 1998, New York: United Nations. 68 p.

4. National Institute on Drug Abuse. Overdose Death Rates. 2017 [cited 2018 March 13]; Available from: https://www.drugabuse.gov/related-topics/trends-statistics/overdose-death-rates.

5. Besacier, F., et al., Comparative chemical analyses of drug samples: general approach and application to heroin. Forensic Science International, 1997. 85(2): p. 113-125.

6. Neumann, H., Comparison of heroin by capillary gas chromatography in Germany. Forensic Science International, 1994. 69(1): p. 7-16.

7. Myors, R., et al., Preliminary investigation of heroin fingerprinting using trace element concentrations. Analytical Communications, 1998. 35: p. 403-410.

8. Myors, R., et al., Investigation of heroin profiling using trace organic impurities. Analyst, 2001. 126(5): p. 679-689.

9. Gröger, T., et al., Application of two-dimensional gas chromatography combined with pixel-based chemometric processing for the chemical profiling of illicit drug samples. Journal of Chromatography A, 2008. 1200(1): p. 8-16.

10. Lurie, I.S., et al., Use of dynamically coated capillaries with added cyclodextrins for the analysis of opium using capillary electrophoresis. Journal of Chromatography A, 2003. 984(1): p. 109-120.

11. Lurie, I.S., et al., Use of dynamically coated capillaries for the determination of heroin, basic impurities and adulterants with capillary electrophoresis. Journal of Chromatography A, 2004. 1034(1): p. 227-235.

12. Lurie, I.S. and S.M. Carr, The Quantitation of Heroin and Selected Basic Impurities Via Reversed Phase HPLC. I. The Analysis of Unadulterated Heroin Samples. Journal of Liquid Chromatography, 1986. 9(11): p. 2485-2509.

13. Lurie, I.S. and K. McGuinness, The Quantitation of Heroin and Selected Basic Impurities via Reversed Phase HPLC. II. The Analysis of Adulterated Samples. Journal of Liquid Chromatography, 1987. 10(10): p. 2189-2204.

14. Lurie, I.S. and S.G. Toske, Applicability of ultra-performance liquid chromatography-tandem mass spectrometry for heroin profiling. Journal of Chromatography A, 2008. 1188(2): p. 322-326. 
15. Morello, D.R., et al., Signature Profiling and Classification of Illicit Heroin by GCMS Analysis of Acidic and Neutral Manufacturing Impurities. Journal of Forensic Sciences, 2010. 55(1): p. 42-49.

16. Chiarotti, M. and N. Fucci, Analysis of volatile compounds in heroin samples. Forensic Science International, 1988. 37(1): p. 47-53.

17. Perillo, B.A., R.F.X. Klein, and E.S. Franzosa, Recent advances by the U.S. Drug Enforcement Administration in drug signature and comparative analysis. Forensic Science International, 1994. 69(1): p. 1-6.

18. Morello, D. and R. Meyers, Qualitative and Quantitative Determination of Residual Solvents in Illicit Cocaine $\mathrm{HCl}$ and Heroin $\mathrm{HCl}$. Journal of Forensic Sciences, 1995. 40(6): p. 957-963.

19. Ehleringer, J.R., et al., Geo-location of heroin and cocaine by stable isotope ratios. Forensic Science International, 1999. 106: p. 27-35.

20. Casale, J.F., et al., Isotopic fractionation of carbon and nitrogen during the illicit processing of cocaine and heroin in South America. Journal of Forensic Sciences, 2005. 50(6): p. 1315-1321.

21. Casale, J., et al., Stable isotope analyses of heroin seized from the merchant vessel Pong Su. Journal of Forensic Sciences, 2006. 51(3): p. 603-606.

22. Collins, M., et al., Chemical profiling of heroin recovered from the North Korean merchant vessel Pong Su. Journal of Forensic Sciences, 2006. 51(3): p. 597-602.

23. Casale, J.F., S.G. Toske, and P.A. Hays, Chlorinated opium alkaloid derivatives produced by the use of aqueous sodium hypochlorite during the clandestine manufacture of heroin. Journal of Forensic Sciences, 2009. 54(2): p. 359-364.

24. Casale, J., J. Moore, and D. Cooper, Novel Chlorinated Tropanes Derived from the Treatment of Cocaine with Sodium Hypochlorite. Journal of Forensic Sciences, 1995. 40(5).

25. Compton, W.M., C.M. Jones, and G.T. Baldwin, Relationship between Nonmedical Prescription-Opioid Use and Heroin Use. N Engl J Med, 2016. 374(2): p. 154-63.

26. Lurie, I.S., et al., Profiling of illicit fentanyl using UHPLC-MS/MS. Forensic Science International, 2012. 220(1): p. 191-196.

27. Mallette, J.R., et al., Characterization of (2R, $4 S)$ - and (2R,4R)-2-Methylfentanyl and their differentiation from cis- and trans-3-Methylfentanyl. Forensic Chemistry, 2018. 8: p. 64-71.

28. Casale, J.F., J.R. Mallette, and E.M. Guest, Analysis of illicit carfentanil: Emergence of the death dragon. Forensic Chemistry, 2017. 3: p. 74-80.

29. 2017 National Drug Threat Assessment. 2017, United States Drug Enforcement Administration, Strategic Intelligence Section: https://www.dea.gov. 
30. Desage, M., et al., Gas-Chromatography with Mass-Spectrometry or Isotope-Ratio Mass-Spectrometry in Studying the Geographical Origin of Heroin. Analytica Chimica Acta, 1991. 247(2): p. 249-254.

31. Dautraix, S., et al., 13C Isotopic analysis of an acetaminophen and diacetylmorphine mixture. Journal of Chromatography A, 1996. 756: p. 203-210.

32. Zhang, D., et al., Origin differentiation of a heroin sample and its acetylating agent with C-13 isotope ratio mass spectrometry. European Journal of Mass Spectrometry, 2005. 11(3): p. 277-285.

33. Toske, S.G., et al., Neutral Heroin Impurities from Tetrahydrobenzylisoquinoline Alkaloids. Journal of Forensic Sciences, 2006. 51(2): p. 308-320.

34. Lurie, I.S., et al., Determination of heroin and basic impurities for drug profiling by ultra-high-pressure liquid chromatography. Forensic Science International, 2013. 231(1-3): p. 300-305.

35. Liu, C., Z. Hua, and Y. Bai, Classification of illicit heroin by UPLC-Q-TOF analysis of acidic and neutral manufacturing impurities. Forensic Science International, 2015. 257: p. 196-202.

36. Bora, T., M. Merdivan, and C. Hamamci, Levels of trace and major elements in illicit heroin. Journal of Forensic Sciences, 2002. 47(5): p. 959-963.

37. Budic, B. and S. Klemenc, Determination of trace elements in heroin by inductively coupled plasma atomic emission spectrometry using ultrasonic nebulization. Spectrochimica Acta Part B: Atomic Spectroscopy, 2000. 55: p. 681-688.

38. Chan, K.-W., et al., Street-level classification of illicit heroin using inorganic elements coupled with pattern monitoring. Egyptian Journal of Forensic Sciences, 2016. 6(3): p. 275-279.

39. Liu, C., et al., Profiling and classification of illicit heroin by ICP-MS analysis of inorganic elements. Forensic Science International, 2014. 239: p. 37-43.

40. Chan, K.-W., et al., A recommended procedure for establishing the source level relationships between heroin case samples of unknown origins. Egyptian Journal of Forensic Sciences, 2014. 4(2): p. 45-49.

41. Chan, K.-W., G.H. Tan, and R.C.S. Wong, Investigation of trace inorganic elements in street doses of heroin. Science and Justice, 2013. 53: p. 73-80.

42. Chan, K.-W., G.-H. Tan, and R.C. Wong, ICP-MS Method Validation for the Analysis of Trace Elements in Illicit Heroin. Analytical Letters, 2012. 45: p. 11221132.

43. Skoog, D., F.J. Holler, and S. Crouch, Principles of Instrumental Analysis. 2007: Thomson Brooks/Cole.

44. Pupyshev, A.A. and E.V. Semenova, Formation of doubly charged atomic ions in the inductively coupled plasma. Spectrochimica Acta Part B: Atomic Spectroscopy, 2001. 56(12): p. 2397-2418. 
45. Microwave-Enhanced Chemistry, ed. H.M. Kingston and S.J. Haswell. 1997, Washington, DC: American Chemical Society. 66-82.

46. ASTM International., ASTM E1169-17 Standard Practice for Conducting Ruggedness Tests. 2017.

47. USP Technologies., Nitrogen Oxides (NOx) Abatement with Hydrogen Peroxide, in Industrial Technical Bulletin, 2015.

48. Element 2 High Resolution ICP-MS Hardware Training Presentation. 2007, ThermoFisher Scientific.

49. Miller, J.N. and J.C. Miller, Statistics and Chemometrics for Analytical Chemistry. 6 ed. 2010, Harlow, England: Pearson Eduction Limited.

50. Hu, Z., et al., Rare Earth Elements in Soils. Communications in Soil Science and Plant Analysis, 2006. 37(9-10): p. 1381-1420.

51. Almirall, J., Elemental Analysis of Glass Fragments, in Forensic examination of glass and paint: analysis and interpretation, B. Caddy, Editor. 2001, New York : Taylor \& Francis, 2001.

52. Campbell, G.P. and J.M. Curran, The interpretation of elemental composition measurements from forensic glass evidence III. Science \& Justice, 2009. 49: p. 27.

53. Houck, M.M., Mute Witnesses: Trace Evidence Analysis. 2001, San Diego, Calif. ; London: Academic Press;.

54. Jantzi, S.C., Elemental Analysis and Forensic Comparison of Soils by LaserInduced Breakdown Spectroscopy (LIBS) and Laser Ablation Inductively Coupled Plasma Mass Spectrometry (LA-ICP-MS). 2013: FIU Electronic Theses and Dissertations. 967.

55. Uitdehaag, S., et al., Forensic Comparison of Soil Samples Using Nondestructive Elemental Analysis. Journal of Forensic Sciences, 2017. 62(4): p. 861-868.

56. Dettman, J.R., et al., Forensic Discrimination of Copper Wire Using Trace Element Concentrations. Analytical Chemistry, 2014. 86(16): p. 8176-8182.

57. Ulrich, A., et al., ICP-MS trace-element analysis as a forensic tool. Analytical \& Bioanalytical Chemistry, 2004. 378(4): p. 1059-1068.

58. Bolck, A., H. Ni, and M. Lopatka, Evaluating score- and feature-based likelihood ratio models for multivariate continuous data: applied to forensic MDMA comparison. Law, Probability \& Risk, 2015. 14(3): p. 243-266.

59. Hays, P.A. and I.S. Lurie, Quantitative Analysis of Adulterants in Illicit Heroin Samples via Reversed Phase HPLC. Journal of Liquid Chromatography, 1991. 14(19): p. 3513-3517.

60. Huizer, H., Analytical Studies on Illicit Heroin II. Comparison of Samples. Journal of Forensic Sciences, 1983. 28(1): p. 40-48. 
61. Klemenc, S., In common batch searching of illicit heroin samples - evaluation of data by chemometrics methods. Forensic Science International, 2001. 115(1): p. 43 52.

62. DeBord, J., et al., Profiling of heroin and assignment of provenance by ${ }^{87} \mathrm{Sr} /{ }^{86} \mathrm{Sr}$ isotope ratio analysis. Inorganica Chimica Acta, 2017. 468(1): p. 292-299.

63. ASTM International, ASTM E2927-16e1 Standard Test Method for Determination of Trace Elements in Soda-Lime Glass Samples Using Laser Ablation Inductively Coupled Plasma Mass Spectrometry for Forensic Comparisons. 2016.

64. $\quad R$ Core Team (2018). R: A language and environment for statistical computing. $R$ Foundation for Statistical Computing, Vienna, Austria. URL https://www.Rproject.org/.

65. Microsoft Corporation and Steve Weston (2017). doParallel: Foreach Parallel Adaptor for the 'parallel' Package. $R$ package version 1.0.11. https://CRAN.Rproject.org/package $=$ doParallel.

66. Microsoft and Steve Weston (2017). foreach: Provides Foreach Looping Construct for $R$. $R$ package version 1.4.4. https://CRAN.R-project.org/package=foreach.

67. Alexander Walker (2017). openxlsx: Read, Write and Edit XLSX Files. R package version 4.0.17. https://CRAN.R-project.org/package=openxlsx.

68. Morello, D.R. and R.P. Meyers, Qualitative and Quantitative Determination of Residual Solvents in Illicit Cocaine $\mathrm{HCl}$ and Heroin $\mathrm{HCl}$. Journal of Forensic Sciences (Wiley-Blackwell), 1995. 40(6): p. 957-963.

69. Idoine, F.A., J.F. Carter, and R. Sleeman, Bulk and compound-specific isotopic characterisation of illicit heroin and cling film. Rapid Communications in Mass Spectrometry, 2005. 19(22): p. 3207-3215.

70. Hays, P.A., et al., Geographic origin determination of heroin and cocaine using site-specific isotopic ratio deuterium NMR. Journal of Forensic Sciences, 2000. 45(3): p. 552-562.

71. Bong, Y.-S., et al., Determining the geographical origin of Chinese cabbages using multielement composition and strontium isotope ratio analyses. Food Chemistry, 2012. 135(4): p. 2666-2674.

72. Lee, A.R., et al., A Multianalytical Approach for Determining the Geographical Origin of Ginseng Using Strontium Isotopes, Multielements, and ${ }^{1} H$ NMR Analysis. Journal of Agricultural and Food Chemistry, 2011. 59(16): p. 8560-8567.

73. Swoboda, S., et al., Identification of Marchfeld asparagus using $\mathrm{Sr}$ isotope ratio measurements by MC-ICP-MS. Analytical and Bioanalytical Chemistry, 2008. 390(2): p. 487-494.

74. Song, B.-Y., et al., Effects of bedrock on the chemical and Sr isotopic compositions of plants. Environmental Earth Sciences, 2015. 74(1): p. 829-837. 
75. Bataille, C.P. and G.J. Bowen, Mapping ${ }^{87} \mathrm{Sr} /{ }^{86} \mathrm{Sr}$ variations in bedrock and water for large scale provenance studies. Chemical Geology, 2012. 304: p. 39-52.

76. Bataille, C.P., J. Laffoon, and G.J. Bowen, Mapping multiple source effects on the strontium isotopic signatures of ecosystems from the circum-Caribbean region. Ecosphere, 2012. 3(12): p. 1-24.

77. Crowley, B.E., J.H. Miller, and C.P. Bataille, Strontium isotopes $\left({ }^{87} \mathrm{Sr} /{ }^{86} \mathrm{Sr}\right)$ in terrestrial ecological and palaeoecological research: empirical efforts and recent advances in continental-scale models. Biological Reviews, 2017. 92(1): p. 43-59.

78. Degryse, P., et al., Considerations on the provenance determination of plant ash glasses using strontium isotopes. Journal of Archaeological Science, 2010. 37(12): p. 3129-3135.

79. Pourmand, A., N. Dauphas, and T.J. Ireland, A novel extraction chromatography and MC-ICP-MS technique for rapid analysis of REE, Sc and Y: Revising CIchondrite and Post-Archean Australian Shale (PAAS) abundances. Chemical Geology, 2012. 291: p. 38-54.

80. Horwitz, E.P., R. Chiarizia, and M.L. Dietz, A novel strontium-selective extraction chromatographic resin. Solvent Extraction and Ion Exchange, 1992. 10(2): p. 313336.

81. Russell, W.A., D.A. Papanastassiou, and T.A. Tombrello, Ca isotope fractionation on the Earth and other solar system materials. Geochimica et Cosmochimica Acta, 1978. 42(8): p. 1075-1090.

82. Weis, D., et al., High-precision isotopic characterization of USGS reference materials by TIMS and MC-ICP-MS. Geochemistry, Geophysics, Geosystems, 2006. 7(8): p. n/a-n/a.

83. Pourmand, A., J.M. Prospero, and A. Sharifi, Geochemical fingerprinting of transAtlantic African dust based on radiogenic Sr-Nd-Hf isotopes and rare earth element anomalies. Geology, 2014. 42(8): p. 675-678.

84. Discriminant Analysis. Multivariate Methods 2018 [cited 2018 May 5]; Available from: https://www.jmp.com/support/help/14/discriminant-analysis-3.shtml\#.

85. Sah, R.N. and P.H. Brown, Isotope ratio determination in boron analysis. Biological Trace Element Research, 1998. 66(1): p. 39-53.

86. Kakihana, H., et al., Fundamental Studies on the Ion-Exchange Separation of Boron Isotopes. Bulletin of the Chemical Society of Japan, 1977. 50(1): p. 158-163.

87. HSDB: Nitric Acid CASRN: 7697-37-2. 2/14/2012 [cited 2018 May 2nd]; Available from: https://toxnet.nlm.nih.gov/cgi-bin/sis/search2/r?dbs+hsdb:@term+@rn+@rel+7697-37-2.

88. Pawliszyn, J., Applications of solid phase microextraction, in RSC chromatography monographs. 1999, Royal Society of Chemistry: Cambridge. 
89. Almirall, J.F., Wen, Capillary microextractor of volatiles (CMV). 2016, Florida International University: United States.

90. Fan, W. and J. Almirall, High-efficiency headspace sampling of volatile organic compounds in explosives using capillary microextraction of volatiles (CMV) coupled to gas chromatography-mass spectrometry (GC-MS). Analytical \& Bioanalytical Chemistry, 2014. 406(8): p. 2189-2195.

91. Guerra-Diaz, P., S. Gura, and J.R. Almirall, Dynamic planar solid phase microextraction--ion mobility spectrometry for rapid field air sampling and analysis of illicit drugs and explosives. Analytical Chemistry, 2010. 82(7): p. 2826.

92. Gura, S., et al., Capillary microextraction of volatiles device for enhanced BTEX vapors sampling based on a phenyl modified PDMS sol-gel adsorption phase. Analytica Chimica Acta, 2018. 1014: p. 27-40.

93. Wiebelhaus, N., et al., Differentiation of marijuana headspace volatiles from other plants and hemp products using capillary microextraction of volatiles (CMV) coupled to gas-chromatography-mass spectrometry $(G C-M S)$. Forensic Chemistry, 2016. 2: p. 1-8.

94. Fan, W., Improved Dynamic Headspace Sampling and Detection using Capillary Microextraction of Volatiles Coupled to Gas Chromatography Mass Spectrometry. 2013, FIU Electronic Theses and Dissertations. 982.

95. Tarifa, A. and J.R. Almirall, Fast detection and characterization of organic and inorganic gunshot residues on the hands of suspects by CMV-GC-MS and LIBS. Science \& Justice, 2015. 55: p. 168-175.

96. Nair, M.V. and G.M. Miskelly, Capillary microextraction: A new method for sampling methamphetamine vapour. Forensic Science International, 2016. 268: p. 131-138.

97. Tarifa, A., et al., Cryofocusing capillary microextraction of volatiles (Cryo-CMV) as a novel headspace extraction device for the analysis of volatile organic compounds and smokeless powders. Forensic Chemistry, 2017. 3: p. 81-89.

98. Simonsen, J.L., The constituents of Indian turpentine from Pinus longifolia, Roxb. Part I. Journal of the Chemical Society, Transactions, 1920. 117: p. 570-578.

99. Chan, K.-W., G.-H. Tan, and R.C.S. Wong, Gas Chromatographic Method Optimization and Statistical Validation for the Determination of Trace Impurities in Street Doses of Heroin. Analytical Letters, 2012. 45(10): p. 1156-1171. 
VITA

\section{JOSHUA DEBORD}

Born, Alexandria, Louisiana

2001-2005

B.A., Anthropology

Louisiana State University

Baton Rouge, Louisiana

2006-2009

Housing Advisor

The Road Home Program

New Orleans, Louisiana

2009-2011

Team Leader

Miami-Dade Housing Voucher Program

Miami, Florida

2011-2014

B.S., Chemistry

Florida International University

Miami, Florida

2014-2017

M.S., Chemistry

Florida International University

Miami, Florida

$2017-2018$

Doctoral Candidate

Florida International University

Miami, Florida

Teaching Assistant

Florida International University

Miami, Florida

Research Assistant

Florida International University

Miami, Florida

Dissertation Year Fellow

Florida International University

Miami, Florida

2018-

Forensic Scientist

North Louisiana Criminalistics Laboratory

Shreveport, Louisiana 


\section{PUBLICATIONS AND PRESENTATIONS}

DeBord, J., Pourmand, A., Jantzi, S., Panicker, S., Almirall, J., (2018) Predicting the Origin of Heroin by Analysis of Inorganic Elements and Isotope Ratios of Strontium (Sr), Paper presented at the $70^{\text {Th }}$ Annual Meeting of the American Academy of Forensic Sciences, February 23, 2018; Seattle, WA

DeBord, J., Pourmand, A., Jantzi, S., Panicker, S., Almirall, J., (2017) Profiling of Heroin and Assignment of Provenance by $87 \mathrm{Sr} / 86 \mathrm{Sr}$ Isotope Ratio Analysis. Inorganica Chimica Acta 2017, 468, 294-299

DeBord, J., Pourmand, A., Almirall, J., (2017) Discrimination of Mexican and South American Heroin $\mathrm{HCl}$ with ${ }^{87} \mathrm{Sr} /{ }^{86} \mathrm{Sr}$ Isotopic Analysis, Poster presented at the $21^{\text {st }}$ International Association of Forensic Sciences (IAFS 2017), August 25, 2017; Toronto, Canada

DeBord, J., Pourmand, A., Almirall, J., Characterization of Strontium Isotope Ratios of Mexican and South American Heroin for Origin Determination, Paper presented at the $16^{\text {th }}$ International Forensic Research Institute Symposium, May 10, 2017; Miami, FL.

DeBord, J., Kreitals, N., Almirall, J., Detecting the Presence of Ignitable Liquids and Residues in Suspected Arson Cases by Capillary Microextraction of Volatiles (CMV), Poster presented at the $15^{\text {th }}$ International Forensic Research Institute Symposium, March 15, 2016; Miami, FL.

Roncancio, D.; Yu, H.; Xu, X.; Wu, S.; Liu, R.; DeBord, J.; Lou, X.; Xiao, Y. (2014) A Label-Free Aptamer-Fluorophore Assembly for Rapid and Specific Detection of Cocaine in Biofluids. Anal. Chem. 2014, 86, 11100-11110 